\title{
MEMOIRS of the
} American Mathematical Society

\section{Number 1022}

\section{The Hermitian Two Matrix Model with an Even Quartic Potential}

\author{
Maurice Duits \\ Arno B. J. Kuijlaars \\ Man Yue Mo
}

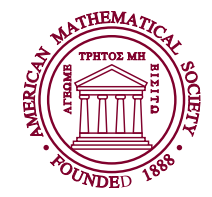




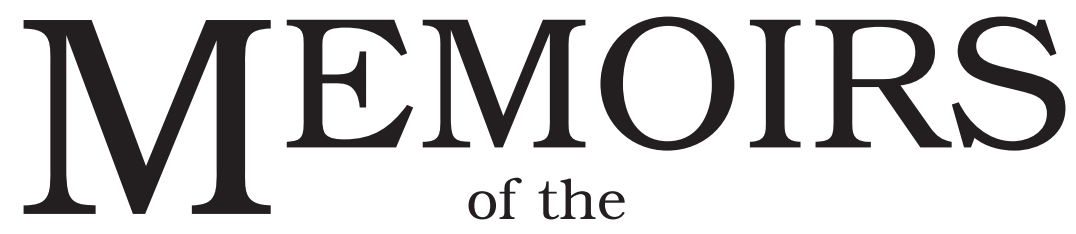

American Mathematical Society

Number 1022

\title{
The Hermitian \\ Two Matrix Model \\ with an Even Quartic Potential
}

\author{
Maurice Duits \\ Arno B. J. Kuijlaars \\ Man Yue Mo
}

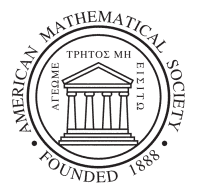

\section{American Mathematical Society}

Providence, Rhode Island 


\section{Library of Congress Cataloging-in-Publication Data}

Duits, Maurice.

The Hermitian two matrix model with an even quartic potential / Maurice Duits, Arno B. J. Kuijlaars, Man Yue Mo.

p. cm. - (Memoirs of the American Mathematical Society, ISSN 0065-9266 ; no. 1022)

"May 2012, volume 217, number 1022 (end of volume)."

Includes bibliographical references and index.

ISBN 978-0-8218-6928-4 (alk. paper)

1. Boundary value problems. 2. Hermitian structures. 3. Eigenvalues. 4. Random matrices. I. Kuijlaars, Arno B. J., 1963- $\quad$ II. Mo, Man Yue. $\quad$ III. Title.

QA379.D85 2011

$512.7^{\prime} 4-\mathrm{dc} 23$

2011051784

\section{Memoirs of the American Mathematical Society}

This journal is devoted entirely to research in pure and applied mathematics.

Publisher Item Identifier. The Publisher Item Identifier (PII) appears as a footnote on the Abstract page of each article. This alphanumeric string of characters uniquely identifies each article and can be used for future cataloguing, searching, and electronic retrieval.

Subscription information. Beginning with the January 2010 issue, Memoirs is accessible from www.ams.org/journals. The 2012 subscription begins with volume 215 and consists of six mailings, each containing one or more numbers. Subscription prices are as follows: for paper delivery, US\$772 list, US\$617.60 institutional member; for electronic delivery, US\$679 list, US $\$ 543.20$ institutional member. Upon request, subscribers to paper delivery of this journal are also entitled to receive electronic delivery. If ordering the paper version, subscribers outside the United States and India must pay a postage surcharge of US\$69; subscribers in India must pay a postage surcharge of US\$95. Expedited delivery to destinations in North America US\$61; elsewhere US\$167. Subscription renewals are subject to late fees. See www . ams.org/help-faq for more journal subscription information. Each number may be ordered separately; please specify number when ordering an individual number.

Back number information. For back issues see www.ams.org/bookstore.

Subscriptions and orders should be addressed to the American Mathematical Society, P. O. Box 845904, Boston, MA 02284-5904 USA. All orders must be accompanied by payment. Other correspondence should be addressed to 201 Charles Street, Providence, RI 02904-2294 USA.

Copying and reprinting. Individual readers of this publication, and nonprofit libraries acting for them, are permitted to make fair use of the material, such as to copy a chapter for use in teaching or research. Permission is granted to quote brief passages from this publication in reviews, provided the customary acknowledgment of the source is given.

Republication, systematic copying, or multiple reproduction of any material in this publication is permitted only under license from the American Mathematical Society. Requests for such permission should be addressed to the Acquisitions Department, American Mathematical Society, 201 Charles Street, Providence, Rhode Island 02904-2294 USA. Requests can also be made by e-mail to reprint-permission@ams.org.

Memoirs of the American Mathematical Society (ISSN 0065-9266) is published bimonthly (each volume consisting usually of more than one number) by the American Mathematical Society at 201 Charles Street, Providence, RI 02904-2294 USA. Periodicals postage paid at Providence, RI. Postmaster: Send address changes to Memoirs, American Mathematical Society, 201 Charles Street, Providence, RI 02904-2294 USA.

(c) 2011 by the American Mathematical Society. All rights reserved.

Copyright of individual articles may revert to the public domain 28 years

after publication. Contact the AMS for copyright status of individual articles.

This publication is indexed in Science Citation Index ${ }^{\circledR}$, SciSearch ${ }^{\circledR}$, Research Alert ${ }^{\circledR}$,

CompuMath Citation Index ${ }^{\circledR}$, Current Contents ${ }^{\circledR} /$ Physical, Chemical 8 Earth Sciences.

Printed in the United States of America.

( The paper used in this book is acid-free and falls within the guidelines established to ensure permanence and durability.

Visit the AMS home page at http://www.ams.org/

$10987654321 \quad 171615141312$ 


\section{Contents}

Chapter 1. Introduction and Statement of Results

1.1. Hermitian two matrix model

1.2. Background

1.3. Vector equilibrium problem

1.4. Solution of vector equilibrium problem

1.5. Classification into cases

1.6. Limiting mean eigenvalue distribution

1.7. About the proof of Theorem 1.4

1.8. Singular cases

Chapter 2. Preliminaries and the Proof of Lemma 1.2

2.1. Saddle point equation and functions $s_{j}$

2.2. Values at the saddles and functions $\theta_{j}$

2.3. Large $z$ asymptotics

2.4. Two special integrals

2.5. Proof of Lemma 1.2

Chapter 4. A Riemann Surface

4.1. The $g$-functions

4.2. Riemann surface $\mathcal{R}$ and $\xi$-functions

4.3. Properties of the $\xi$ functions

4.4. The $\lambda$ functions

Chapter 5. Pearcey Integrals and the First Transformation

5.1. Definitions

5.2. Large $z$ asymptotics

5.3. First transformation: $Y \mapsto X \quad 45$

5.4. RH problem for $X$

Chapter 6. Second Transformation $X \mapsto U \quad 53$

6.1. Definition of second transformation

6.2. Asymptotic behavior of $U$ 
6.3. Jump matrices for $U$

6.4. $\mathrm{RH}$ problem for $U$

Chapter 7. Opening of Lenses

7.1. Third transformation $U \mapsto T$

7.2. $\mathrm{RH}$ problem for $T$

7.3. Jump matrices for $T$

7.4. Fourth transformation $T \mapsto S$

7.5. RH problem for $S$

7.6. Behavior of jumps as $n \rightarrow \infty$

Chapter 8. Global Parametrix

8.1. Statement of $\mathrm{RH}$ problem

8.2. Riemann surface as an $M$-curve

8.3. Canonical homology basis

8.4. Meromorphic differentials

8.5. Definition and properties of functions $u_{j}$

8.6. Definition and properties of functions $v_{j}$

8.7. The first row of $M$

Chapter 9. Local Parametrices and Final Transformation

9.1. Local parametrices

9.2. Final transformation

9.3. Proof of Theorem 1.4

Bibliography

Index 


\begin{abstract}
We consider the two matrix model with an even quartic potential $W(y)=$ $y^{4} / 4+\alpha y^{2} / 2$ and an even polynomial potential $V(x)$. The main result of the paper is the formulation of a vector equilibrium problem for the limiting mean density for the eigenvalues of one of the matrices $M_{1}$. The vector equilibrium problem is defined for three measures, with external fields on the first and third measures and an upper constraint on the second measure. The proof is based on a steepest descent analysis of a $4 \times 4$ matrix valued Riemann-Hilbert problem that characterizes the correlation kernel for the eigenvalues of $M_{1}$. Our results generalize earlier results for the case $\alpha=0$, where the external field on the third measure was not present.
\end{abstract}

Received by the editor October 20, 2010.

Article electronically published on September 20, 2011; S 0065-9266(2011)00639-8.

2010 Mathematics Subject Classification. Primary 30E25, 60B20; Secondary 15B52, 30F10, 31A05, 42C05, 82B26.

Key words and phrases. Two matrix model, eigenvalue distribution, correlation kernel, vector equilibrium problem, Riemann-Hilbert problem, steepest descent analysis.

M. Duits and A.B.J. Kuijlaars are grateful for the support and hospitality of MSRI in Berkeley in the fall of 2010 .

A.B.J. Kuijlaars is supported by K.U. Leuven research grant OT/08/33, FWO-Flanders project G.0427.09 and G.0641.11, by the Belgian Interuniversity Attraction Pole P06/02, and by grant MTM2008-06689-C02-01 of the Spanish Ministry of Science and Innovation.

M. Y. Mo acknowledges financial support by the EPSRC grant EP/G019843/1.

Author affiliations at time of publication: Maurice Duits, Department of Mathematics, California Institute of Technology, 1200 E. California Blvd, Pasadena California 91125, email: mduits@caltech.edu and Department of Mathematics, KTH Royal Institute of Technology, SE10044 Stockholm, Sweden, email: duits@kth.se; Arno B.J. Kuijlaars, Department of Mathematics, Katholieke Universiteit Leuven, Celestijnenlaan 200B, 3001 Leuven, Belgium, email: arno.kuijlaars@wis.kuleuven.be; and Man Yue Mo, Department of Mathematics, University of Bristol, Bristol BS8 1TW, United Kingdom, email: m.mo@bristol.ac.uk. 


\section{CHAPTER 1}

\section{Introduction and Statement of Results}

\subsection{Hermitian two matrix model}

The Hermitian two matrix model is a probability measure of the form

$$
\frac{1}{Z_{n}} \exp \left(-n \operatorname{Tr}\left(V\left(M_{1}\right)+W\left(M_{2}\right)-\tau M_{1} M_{2}\right)\right) d M_{1} d M_{2},
$$

defined on the space of pairs $\left(M_{1}, M_{2}\right)$ of $n \times n$ Hermitian matrices. The constant $Z_{n}$ in (1.1) is a normalization constant, $\tau \in \mathbb{R} \backslash\{0\}$ is the coupling constant and $d M_{1} d M_{2}$ is the flat Lebesgue measure on the space of pairs of Hermitian matrices. In (1.1), $V$ and $W$ are the potentials of the matrix model. In this paper, we assume $V$ to be a general even polynomial and we take $W$ to be the even quartic polynomial

$$
W(y)=\frac{1}{4} y^{4}+\frac{\alpha}{2} y^{2}, \quad \alpha \in \mathbb{R} .
$$

Without loss of generality we may (and do) assume that

$$
\tau>0 \text {. }
$$

We are interested in describing the eigenvalues of $M_{1}$ in the large $n$ limit.

In 45 the case $\alpha=0$ was studied in detail. An important ingredient in the analysis of [45] was a vector equilibrium problem that describes the limiting mean eigenvalue distribution of $M_{1}$. In this paper we extend the vector equilibrium problem to the case $\alpha \neq 0$.

\subsection{Background}

The two matrix model (1.1) with polynomial potentials $V$ and $W$ was introduced in [59, 71] as a model for quantum gravity and string theory. The interest is in the double scaling limit for critical potentials. It is generally believed that the two matrix model is able to describe all $(p, q)$ conformal minimal models, whereas the one matrix model is limited to $(p, 2)$ minimal models [30, 41, 48. In 62 the two matrix model was proposed for the study of the Ising model on a random surface, where the logarithm of the partition function (i.e., the normalizing constant $Z_{n}$ in (1.1)) is expected to be the generating function in the enumeration of graphs on surfaces. For more information and background on the physical interest we refer to the the surveys $[\mathbf{3 9}, \mathbf{4 0}$, and more recent physical papers $[\mathbf{9}, \mathbf{4 9}, \mathbf{5 1}, \mathbf{5 2}$

The two matrix model has a very rich integrable structure that is connected to biorthogonal polynomials, isomonodromy deformations, Riemann-Hilbert problems and integrable equations, see e.g. [2, 10, 12, 13, 14, 46, 50, 61, 67. This is the basis of the mathematical treatment of the two matrix model, see also the survey 11]. 
The eigenvalues of the matrices $M_{1}$ and $M_{2}$ in the two matrix model are a determinantal point process with correlation kernels that are expressed in terms of biorthogonal polynomials. These are two families of monic polynomials $\left\{p_{k, n}(x)\right\}_{k=0}^{\infty}$ and $\left\{q_{l, n}(y)\right\}_{y=0}^{\infty}$, where $p_{k, n}$ has degree $k$ and $q_{l, n}$ has degree $l$, satisfying the condition

$$
\int_{-\infty}^{\infty} \int_{-\infty}^{\infty} p_{k, n}(x) q_{l, n}(y) e^{-n(V(x)+W(y)-\tau x y)} d x d y=h_{k, n}^{2} \delta_{k, l} .
$$

The polynomials are well-defined by (1.4) and have simple and real zeros 46. Moreover, the zeros of $p_{k, n}$ and $p_{k+1, n}$, and those of $q_{l, n}$ and $q_{l+1, n}$ are interlacing 43.

The kernels are expressed in terms of these biorthogonal polynomials and their transformed functions

$$
\begin{aligned}
& Q_{l, n}(x)=e^{-n V(x)} \int_{-\infty}^{\infty} q_{l, n}(y) e^{-n(W(y)-\tau x y)} d y, \\
& P_{k, n}(y)=e^{-n W(y)} \int_{-\infty}^{\infty} p_{k, n}(x) e^{-n(V(x)-\tau x y)} d x,
\end{aligned}
$$

as follows:

$$
\begin{aligned}
K_{11}^{(n)}\left(x_{1}, x_{2}\right) & =\sum_{k=0}^{n-1} \frac{1}{h_{k, n}^{2}} p_{k, n}\left(x_{1}\right) Q_{k, n}\left(x_{2}\right), \\
K_{12}^{(n)}(x, y) & =\sum_{k=0}^{n-1} \frac{1}{h_{k, n}^{2}} p_{k, n}(x) q_{k, n}(y), \\
K_{21}^{(n)}(y, x) & =\sum_{k=0}^{n-1} \frac{1}{h_{k, n}^{2}} P_{k, n}(y) Q_{k, n}(x)-e^{-n(V(x)+W(y)-\tau x y)}, \\
K_{22}^{(n)}\left(y_{1}, y_{2}\right) & =\sum_{k=0}^{n-1} \frac{1}{h_{k, n}^{2}} P_{k, n}\left(y_{1}\right) q_{k, n}\left(y_{2}\right) .
\end{aligned}
$$

Then Eynard and Mehta [50, 73, see also [24, 37, 72, showed that the joint probability density function for the eigenvalues $x_{1}, \ldots, x_{n}$ of $M_{1}$ and $y_{1}, \ldots, y_{n}$ of $M_{2}$ is given by

$$
\begin{aligned}
& \mathcal{P}\left(x_{1}, \ldots, x_{n}, y_{1}, \ldots, y_{n}\right) \\
&=\frac{1}{(n !)^{2}} \operatorname{det}\left(\begin{array}{ll}
\left(K_{11}^{(n)}\left(x_{i}, x_{j}\right)\right)_{i, j=1}^{n} & \left(K_{12}^{(n)}\left(x_{i}, y_{j}\right)\right)_{i, j=1}^{n} \\
\left(K_{21}^{(n)}\left(y_{i}, x_{j}\right)\right)_{i, j=1}^{n^{n}} & \left(K_{22}^{(n)}\left(y_{i}, y_{j}\right)\right)_{i, j=1}^{n^{2}}
\end{array}\right),
\end{aligned}
$$

and the marginal densities take the form

$$
\begin{aligned}
& \underbrace{\int \cdots \int}_{n-k+n-l \text { times }} \mathcal{P}\left(x_{1}, \ldots, x_{n}, y_{1}, \ldots, y_{n}\right) d x_{k+1} \cdots d x_{n} d y_{l+1} \cdots d y_{n} \\
& \quad=\frac{(n-k) !(n-l) !}{(n !)^{2}} \operatorname{det}\left(\begin{array}{ll}
\left(K_{11}^{(n)}\left(x_{i}, x_{j}\right)\right)_{i, j=1}^{k} & \left(K_{12}^{(n)}\left(x_{i}, y_{j}\right)\right)_{i, j=1}^{k, l} \\
\left(K_{21}^{(n)}\left(y_{i}, x_{j}\right)\right)_{i, j=1}^{l, k} & \left(K_{22}^{(n)}\left(y_{i}, y_{j}\right)\right)_{i, j=1}^{l}
\end{array}\right) .
\end{aligned}
$$


In particular, by taking $l=0$, so that we average over the eigenvalues $y_{1}, \ldots, y_{n}$ of $M_{2}$, we find that the eigenvalues of $M_{1}$ are a determinantal point process with kernel $K_{11}^{(n)}$, see (1.5). This kernel is constructed out of the biorthogonal family $\left\{p_{k, n}\right\}_{k=0}^{\infty}$ and $\left\{Q_{l, n}\right\}_{l=0}^{\infty}$ and the associated determinantal point process is an example of a biorthogonal ensemble [23. It is also an example of a multiple orthogonal polynomial ensemble in the sense of $6 \mathbf{6 3}$.

In order to describe the behavior of the eigenvalues in the large $n$ limit, one needs to control the kernels (1.5)-(1.8) as $n \rightarrow \infty$. Due to special recurrence relations satisfied by the biorthogonal polynomials, there exist Christoffel-Darbouxtype formulas that express the $n$-term sums (1.5) and (1.8) into a finite number (independent of $n$ ) of biorthogonal polynomials and transformed functions, see 13. This paper also gives differential equations and a remarkable duality between spectral curves, see also [12, 14.

A Riemann-Hilbert problem for biorthogonal polynomials was first formulated in 46]. The Riemann-Hilbert problem in [46] is of size $2 \times 2$ but it is non-local and one has not been able to apply an asymptotic analysis to it. Local Riemann-Hilbert problems were formulated in [14, 61, 67, but these Riemann-Hilbert problems are of larger size, depending on the degrees of the potentials $V$ and $W$. The formulation of a local Riemann-Hilbert problem for biorthogonal polynomials, however, opens up the way for the application of the Deift-Zhou [35] steepest descent method, which was applied very successfully to the Riemann-Hilbert problem for orthogonal polynomials, see [18, 31, 33, 34] and many later papers.

In 45 the Deift-Zhou steepest descent method was indeed applied to the Riemann-Hilbert problem from [67. for the case where $W$ is given by (1.2) with $\alpha=0$. It gave a precise asymptotic analysis of the kernel $K_{11}^{(n)}$ as $n \rightarrow \infty$, leading in particular to the local universality results that are well-known in one matrix models 33. The analysis in [45] was restricted to the genus zero case. The extension to higher genus was done in $\mathbf{7 6}$.

\subsection{Vector equilibrium problem}

As already stated, it is the purpose of the present paper to extend the results of [45, 76] to the case of general $\alpha$.

An important role in the analysis in 45 is played by a vector equilibrium problem that characterizes the limiting mean density for the eigenvalues of $M_{1}$ (and also gives the limiting zero distribution of the biorthogonal polynomials $p_{n, n}$ ). One of the main contributions of the present paper is the formulation of the appropriate generalization to general $\alpha \in \mathbb{R}$. We refer to the standard reference [79] for notions of logarithmic potential theory and equilibrium problems with external fields.

1.3.1. Case $\alpha=0$. Let us first recall the vector equilibrium problem from [45, which involves the minimization of an energy functional over three measures. For a measure $\mu$ on $\mathbb{C}$ we define the logarithmic energy

$$
I(\mu)=\iint \log \frac{1}{|x-y|} d \mu(x) d \mu(y)
$$

and for two measures $\mu$ and $\nu$ we define the mutual energy

$$
I(\mu, \nu)=\iint \log \frac{1}{|x-y|} d \mu(x) d \nu(y) .
$$


The energy functional in 45 , then takes the form

$$
\sum_{j=1}^{3} I\left(\nu_{j}\right)-\sum_{j=1}^{2} I\left(\nu_{j}, \nu_{j+1}\right)+\int\left(V(x)-\frac{3}{4}|\tau x|^{4 / 3}\right) d \nu_{1}(x)
$$

and the vector equilibrium problem is to minimize (1.10) among all measures $\nu_{1}$, $\nu_{2}$ and $\nu_{3}$ such that

(a) the measures have finite logarithmic energy;

(b) $\nu_{1}$ is a measure on $\mathbb{R}$ with $\nu_{1}(\mathbb{R})=1$;

(c) $\nu_{2}$ is a measure on $i \mathbb{R}$ with $\nu_{2}(i \mathbb{R})=2 / 3$;

(d) $\nu_{3}$ is a measure on $\mathbb{R}$ with $\nu_{3}(\mathbb{R})=1 / 3$;

(e) $\nu_{2} \leq \sigma_{2}$ where $\sigma_{2}$ is the unbounded measure with density

$$
\frac{d \sigma_{2}}{|d z|}=\frac{\sqrt{3}}{2 \pi} \tau^{4 / 3}|z|^{1 / 3}, \quad z \in i \mathbb{R}
$$

on the imaginary axis.

A main feature of the vector equilibrium problem is that it involves an external field acting on the first measure as well as an upper constraint (1.11) acting on the second measure. Note that an upper constraint arises typically in the asymptotic analysis of discrete orthogonal polynomials, see e.g. [7, 22, 42, 69, 78. The interaction between the measures in (1.10) is of the Nikishin type where consecutive measures attract each other, but there is no direct interaction between measures $\nu_{i}$ and $\nu_{j}$ if $|i-j| \geq 2$. The notion of a Nikishin system originated in works on Hermite-Padé rational approximation, see [5, 56, 63, 77. Vector equilibrium problems also played a role in the recent papers $[\mathbf{8}, \mathbf{1 5}, \mathbf{1 7}, \mathbf{6 6}$ ] that are related to random matrix theory and $6,64,44,70,85$, that are related to recurrence relations.

1.3.2. General $\alpha$. For general $\alpha \in \mathbb{R}$, the relevant energy functional takes the form

$$
\begin{aligned}
E\left(\nu_{1}, \nu_{2}, \nu_{3}\right)=\sum_{j=1}^{3} I\left(\nu_{j}\right)-\sum_{j=1}^{2} I\left(\nu_{j}, \nu_{j+1}\right) & \\
& +\int V_{1}(x) d \nu_{1}(x)+\int V_{3}(x) d \nu_{3}(x),
\end{aligned}
$$

where $V_{1}$ and $V_{3}$ are certain external fields acting on $\nu_{1}$ and $\nu_{3}$, respectively. The vector equilibrium problem is to minimize $E\left(\nu_{1}, \nu_{2}, \nu_{3}\right)$ among all measures $\nu_{1}, \nu_{2}$, $\nu_{3}$, such that

(a) the measures have finite logarithmic energy;

(b) $\nu_{1}$ is a measure on $\mathbb{R}$ with $\nu_{1}(\mathbb{R})=1$;

(c) $\nu_{2}$ is a measure on $i \mathbb{R}$ with $\nu_{2}(i \mathbb{R})=2 / 3$;

(d) $\nu_{3}$ is a measure on $\mathbb{R}$ with $\nu_{3}(\mathbb{R})=1 / 3$;

(e) $\nu_{2} \leq \sigma_{2}$ where $\sigma_{2}$ is a certain measure on the imaginary axis.

Comparing with (1.10) we see that there is an external field $V_{3}$ acting on the third measure as well. The vector equilibrium problem depends on the input data $V_{1}, V_{3}$, and $\sigma_{2}$ that will be described next. Recall that $V$ is an even polynomial and that $W$ is the quartic polynomial given by (1.2). 
1.3.3. External field $V_{1}$. The external field $V_{1}$ that acts on $\nu_{1}$ is defined by

$$
V_{1}(x)=V(x)+\min _{s \in \mathbb{R}}(W(s)-\tau x s) .
$$

The minimum is attained at a value $s=s_{1}(x) \in \mathbb{R}$ for which $W^{\prime}(s)=\tau x$, that is

$$
s^{3}+\alpha s=\tau x .
$$

For $\alpha \geq 0$, this value of $s$ is uniquely defined by (1.14). For $\alpha<0$ there can be more than one real solution of (1.14). The relevant value is the one that has the same sign as $x$ (since $\tau>0$, see (1.3) $)$. It is uniquely defined, except for $x=0$.

1.3.4. External field $V_{3}$. The external field $V_{3}$ that acts on $\nu_{3}$ is not present if $\alpha \geq 0$. Thus

$$
V_{3}(x) \equiv 0 \quad \text { if } \alpha \geq 0 .
$$

For $\alpha<0$, the external field $V_{3}(x)$ is non-zero only for $x \in\left(-x^{*}(\alpha), x^{*}(\alpha)\right)$ where

$$
x^{*}(\alpha)= \begin{cases}\frac{2}{\tau}\left(\frac{-\alpha}{3}\right)^{3 / 2}, & \alpha<0, \\ 0, & \alpha \geq 0 .\end{cases}
$$

For those $x$, the equation (1.14) has three real solutions $s_{1}=s_{1}(x), s_{2}=s_{2}(x)$, $s_{3}=s_{3}(x)$ which we take to be ordered such that

$$
W\left(s_{1}\right)-\tau x s_{1} \leq W\left(s_{2}\right)-\tau x s_{2} \leq W\left(s_{3}\right)-\tau x s_{3} .
$$

Thus the global minimum of $s \in \mathbb{R} \mapsto W(s)-\tau x s$ is attained at $s_{1}$, and this global minimum played a role in the definition (1.13) of $V_{1}$. The function has another local minimum at $s_{2}$ and a local maximum at $s_{3}$, and these are used in the definition of $V_{3}$. We define $V_{3}: \mathbb{R} \rightarrow \mathbb{R}$ by

$$
V_{3}(x)= \begin{cases}\left(W\left(s_{3}(x)\right)-\tau x s_{3}(x)\right)- & \\ \left(W\left(s_{2}(x)\right)-\tau x s_{2}(x)\right), & \text { for } x \in\left(-x^{*}(\alpha), x^{*}(\alpha)\right), \\ 0 & \text { elsewhere. }\end{cases}
$$

Thus $V_{3}(x)$ is the difference between the local maximum and the other local minimum of $s \in \mathbb{R} \mapsto W(s)-\tau x s$, which indeed exist if and only if $x \in\left(-x^{*}(\alpha), x^{*}(\alpha)\right)$, where $x^{*}(\alpha)$ is given by (1.16). In particular $V_{3}(x)>0$ for $x \in\left(-x^{*}(\alpha), x^{*}(\alpha)\right)$.

1.3.5. The constraint $\sigma_{2}$. To describe the measure $\sigma_{2}$ that acts as a constraint on $\nu_{2}$, we consider the equation

$$
s^{3}+\alpha s=\tau z, \quad \text { with } z \in i \mathbb{R} .
$$

There is always a solution $s$ on the imaginary axis. The other two solutions are either on the imaginary axis as well, or they are off the imaginary axis, and lie symmetrically with respect to the imaginary axis. We define

$$
\frac{d \sigma_{2}(z)}{|d z|}=\frac{\tau}{\pi} \operatorname{Re} s(z)
$$

where $s(z)$ is the solution of (1.18) with largest real part. We then have for the support $S\left(\sigma_{2}\right)$ of $\sigma_{2}$,

$$
S\left(\sigma_{2}\right)=i \mathbb{R} \backslash\left(-i y^{*}(\alpha), i y^{*}(\alpha)\right)
$$


where

$$
y^{*}(\alpha)= \begin{cases}\frac{2}{\tau}\left(\frac{\alpha}{3}\right)^{3 / 2}, & \alpha>0, \\ 0, & \alpha \leq 0 .\end{cases}
$$

This completes the description of the vector equilibrium problem for general $\alpha$. It is easy to check that for $\alpha=0$ it reduces to the vector equilibrium described before.

\subsection{Solution of vector equilibrium problem}

Our first main theorem deals with the solution of the vector equilibrium problem. We use $S(\mu)$ to denote the support of a measure $\mu$. The logarithmic potential of $\mu$ is the function

$$
U^{\mu}(x)=\int \log \frac{1}{|x-s|} d \mu(s), \quad x \in \mathbb{C},
$$

which is a harmonic function on $\mathbb{C} \backslash S(\mu)$ and superharmonic on $\mathbb{C}$.

THEOREM 1.1. The above vector equilibrium problem has a unique minimizer $\left(\mu_{1}, \mu_{2}, \mu_{3}\right)$ that satisfies the following.

(a) There is a constant $\ell_{1} \in \mathbb{R}$ such that

$$
\begin{cases}2 U^{\mu_{1}}(x)=U^{\mu_{2}}(x)-V_{1}(x)+\ell_{1}, & x \in S\left(\mu_{1}\right), \\ 2 U^{\mu_{1}}(x) \geq U^{\mu_{2}}(x)-V_{1}(x)+\ell_{1}, & x \in \mathbb{R} \backslash S\left(\mu_{1}\right) .\end{cases}
$$

If $0 \notin S\left(\mu_{1}\right)$ or $0 \notin S\left(\sigma_{2}-\mu_{2}\right)$ then

$$
S\left(\mu_{1}\right)=\bigcup_{j=1}^{N}\left[a_{j}, b_{j}\right]
$$

for some $N \in \mathbb{N}$ and $a_{1}<b_{1}<a_{2}<\cdots<a_{N}<b_{N}$, and on each of the intervals $\left[a_{j}, b_{j}\right]$ in $S\left(\mu_{1}\right)$ there is a density

$$
\frac{d \mu_{1}}{d x}=\rho_{1}(x)=\frac{1}{\pi} h_{j}(x) \sqrt{\left(b_{j}-x\right)\left(x-a_{j}\right)}, \quad x \in\left[a_{j}, b_{j}\right]
$$

and $h_{j}$ is non-negative and real analytic on $\left[a_{j}, b_{j}\right]$.

(b) We have

$$
\begin{cases}2 U^{\mu_{2}}(x)=U^{\mu_{1}}(z)+U^{\mu_{3}}(x), & x \in S\left(\sigma_{2}-\mu_{2}\right), \\ 2 U^{\mu_{2}}(x)<U^{\mu_{1}}(z)+U^{\mu_{3}}(x), & x \in i \mathbb{R} \backslash S\left(\sigma_{2}-\mu_{2}\right),\end{cases}
$$

and there is a constant $c_{2} \geq 0$ such that

$$
S\left(\mu_{2}\right)=S\left(\sigma_{2}\right), \quad \text { and } \quad S\left(\sigma_{2}-\mu_{2}\right)=i \mathbb{R} \backslash\left(-i c_{2}, i c_{2}\right) .
$$

Moreover, $\sigma_{2}-\mu_{2}$ has a density

$$
\frac{d\left(\sigma_{2}-\mu_{2}\right)}{|d z|}=\rho_{2}(z), \quad z \in i \mathbb{R}
$$

that is positive and real analytic on $i \mathbb{R} \backslash\left[-i c_{2}, i c_{2}\right]$. If $c_{2}>0$, then $\rho_{2}$ vanishes as a square root at $z= \pm i c_{2}$. If $\alpha \geq 0$, then $c_{2}>y^{*}(\alpha)$, where $y^{*}(\alpha)$ is given by (1.21). 
(c) We have

$$
\begin{cases}2 U^{\mu_{3}}(x)=U^{\mu_{2}}(x)-V_{3}(x), & x \in S\left(\mu_{3}\right), \\ 2 U^{\mu_{3}}(x)>U^{\mu_{2}}(x)-V_{3}(x), & x \in \mathbb{R} \backslash S\left(\mu_{3}\right),\end{cases}
$$

and there is a constant $c_{3} \geq 0$ such that

$$
S\left(\mu_{3}\right)=\mathbb{R} \backslash\left(-c_{3}, c_{3}\right) .
$$

Moreover, $\mu_{3}$ has a density

$$
\frac{d \mu_{3}}{d x}=\rho_{3}(x), \quad x \in \mathbb{R},
$$

that is positive and real analytic on $\mathbb{R} \backslash\left[-c_{3}, c_{3}\right]$. If $\alpha \geq 0$, then $c_{3}=0$. If $\alpha<0$, then $c_{3}<x^{*}(\alpha)$ where $x^{*}(\alpha)$ is given by (1.16). If $c_{3}>0$, then $\rho_{3}$ vanishes as a square root at $x= \pm c_{3}$.

(d) All three measures are symmetric with respect to 0 , so that for $j=1,2,3$ we have $\mu_{j}(A)=\mu_{j}(-A)$ for every Borel set $A$.

In part (a) of the theorem it is stated that $S\left(\mu_{1}\right)$ is a finite union of intervals under the condition that $S\left(\mu_{1}\right)$ and $S\left(\sigma_{2}-\mu_{2}\right)$ are disjoint. If this condition is not satisfied then we are in one of the singular cases that will be discussed in Section 1.5 below. However, the condition is not necessary as will be explained in Remark 4.9 below. We chose to include the condition in Theorem 1.1 since the focus of the present paper is on the regular cases.

The conditions (1.23), (1.26), and (1.29) are the Euler-Lagrange variational conditions associated with the vector equilibrium problem. We note the strict inequalities in (1.26) and (1.29). These are consequences of special properties of the constraint $\sigma_{2}$ and the external field $V_{3}$ that are listed in parts (b) and (c) of the following lemma.

LEMma 1.2. The following hold.

(a) Let $\nu_{2}$ be a measure on $i \mathbb{R}$ such that $\nu_{2} \leq \sigma_{2}$. If $0 \notin S\left(\sigma_{2}-\nu_{2}\right)$ then $x \mapsto V_{1}(x)-U^{\nu_{2}}(x)$ is real analytic on $\mathbb{R}$.

(b) The density $\frac{d \sigma_{2}}{|d z|}(i y)=\frac{\tau}{\pi} \operatorname{Re} s(i y)$ (see (1.19) ) is an increasing function for $y>0$.

(c) Let $\alpha<0$. Let $\nu_{2}$ be a measure on $i \mathbb{R}$ of finite logarithmic energy such that $\nu_{2} \leq \sigma_{2}$. Then $x \mapsto V_{3}(\sqrt{x})-U^{\nu_{2}}(\sqrt{x})$ is a decreasing and convex function on $\left(0,\left(x^{*}(\alpha)\right)^{2}\right)$.

Lemma 1.2 is proved in Chapter 2 and the proof of Theorem 1.1 is given in Chapter 3 .

A major role in what follows will be played by functions defined on a compact four-sheeted Riemann surface that we will introduce in chapter 4 . The sheets are connected along the supports $S\left(\mu_{1}\right), S\left(\sigma_{2}-\mu_{2}\right)$ and $S\left(\mu_{3}\right)$ of the minimizing measures for the vector equilibrium problem. The main result of chapter 4 is Proposition 4.8 which says that the function defined by

$$
V^{\prime}(z)-\int \frac{d \mu_{1}(s)}{z-s}
$$

on the first sheet has an extension to a globally meromorphic function on the full Riemann surface. This very special property is due to the special forms of the 
external fields $V_{1}$ and $V_{3}$ and the constraint $\sigma_{2}$, which interact in a very precise way.

\subsection{Classification into cases}

According to Theorem 1.1 the structure of the supports is the same for $\alpha>0$ as it was for $\alpha=0$ in $\left[\mathbf{4 5}\right.$, that is, $S\left(\mu_{3}\right)=\mathbb{R}$ and $S\left(\sigma_{2}-\mu_{2}\right)=i \mathbb{R} \backslash\left(-i c_{2}, i c_{2}\right)$ for some $c_{2}>0$. The supports determine the underlying Riemann surface, and so the case $\alpha>0$ is very similar to the case $\alpha=0$. There are no phase transitions in case $\alpha>0$, except for the possible closing or opening of gaps in the support of $\mu_{1}$. These type of transitions already occur in the one matrix model.

For $\alpha<0$, however, certain new phenomena occur which come from the fact that the external field $V_{3}$ on $\mu_{3}$ (defined in (1.17)) has its maximum at 0 and therefore tends to move $\mu_{3}$ away from 0 . As a result there are cases where $S\left(\mu_{3}\right)$ is no longer the full real axis, but a strict subset (1.30) with $c_{3}>0$.

In addition, it is also possible that $c_{2}=0$ in (1.27) such that $S\left(\sigma_{2}-\mu_{2}\right)$ is the full imaginary axis and the constraint $\sigma_{2}$ is not active. These new phenomena already occur for the simplest case

$$
V(x)=\frac{1}{2} x^{2}
$$

for which explicit calculations were done in 43 based on the coefficients in the recurrence relations satisfied by the biorthogonal polynomials. These calculations lead to the phase diagram shown in Figure 1.1 which is taken from 43 . There are four phases corresponding to the following four cases that are determined by the fact whether 0 is in the support of the measures $\mu_{1}, \sigma_{2}-\mu_{2}, \mu_{3}$ or not:

Case I: $0 \in S\left(\mu_{1}\right), 0 \notin S\left(\sigma_{2}-\mu_{2}\right)$, and $0 \in S\left(\mu_{3}\right)$,

Case II: $0 \notin S\left(\mu_{1}\right), 0 \notin S\left(\sigma_{2}-\mu_{2}\right)$, and $0 \in S\left(\mu_{3}\right)$,

Case III: $0 \notin S\left(\mu_{1}\right), 0 \in S\left(\sigma_{2}-\mu_{2}\right)$, and $0 \notin S\left(\mu_{3}\right)$,

Case IV: $0 \in S\left(\mu_{1}\right), 0 \notin S\left(\sigma_{2}-\mu_{2}\right)$, and $0 \notin S\left(\mu_{3}\right)$.

The four cases correspond to regular behavior of the supports at 0 . There is another regular situation (which does not occur for $V(x)=\frac{1}{2} x^{2}$ ), namely

Case V: $0 \notin S\left(\mu_{1}\right), 0 \notin S\left(\sigma_{2}-\mu_{2}\right)$, and $0 \notin S\left(\mu_{3}\right)$.

The five cases determine the cut structure of the Riemann surface and we will use this classification throughout the paper.

Singular behavior occurs when two consecutive supports intersect at 0 .

Singular supports I: $0 \in S\left(\mu_{1}\right) \cap S\left(\sigma_{2}-\mu_{2}\right), 0 \notin S\left(\mu_{3}\right)$,

Singular supports II: $0 \notin S\left(\mu_{1}\right), 0 \in S\left(\sigma_{2}-\mu_{2}\right) \cap S\left(\mu_{3}\right)$.

There is a multisingular case, when all three supports meet at 0 :

Singular supports III: $0 \in S\left(\mu_{1}\right) \cap S\left(\sigma_{2}-\mu_{2}\right) \cap S\left(\mu_{3}\right)$.

Besides a singular cut structure for the Riemann surface, we can also have a singular behavior of the first measure $\mu_{1}$. These singular cases also appear in the usual equilibrium problem for the one matrix model, see [33], and they are as follows.

Singular interior point for $\mu_{1}$ : The density of $\mu_{1}$ vanishes at an interior point of $S\left(\mu_{1}\right)$.

Singular endpoint for $\mu_{1}$ : The density of $\mu_{1}$ vanishes to higher order than square root at an endpoint of $S\left(\mu_{1}\right)$. 


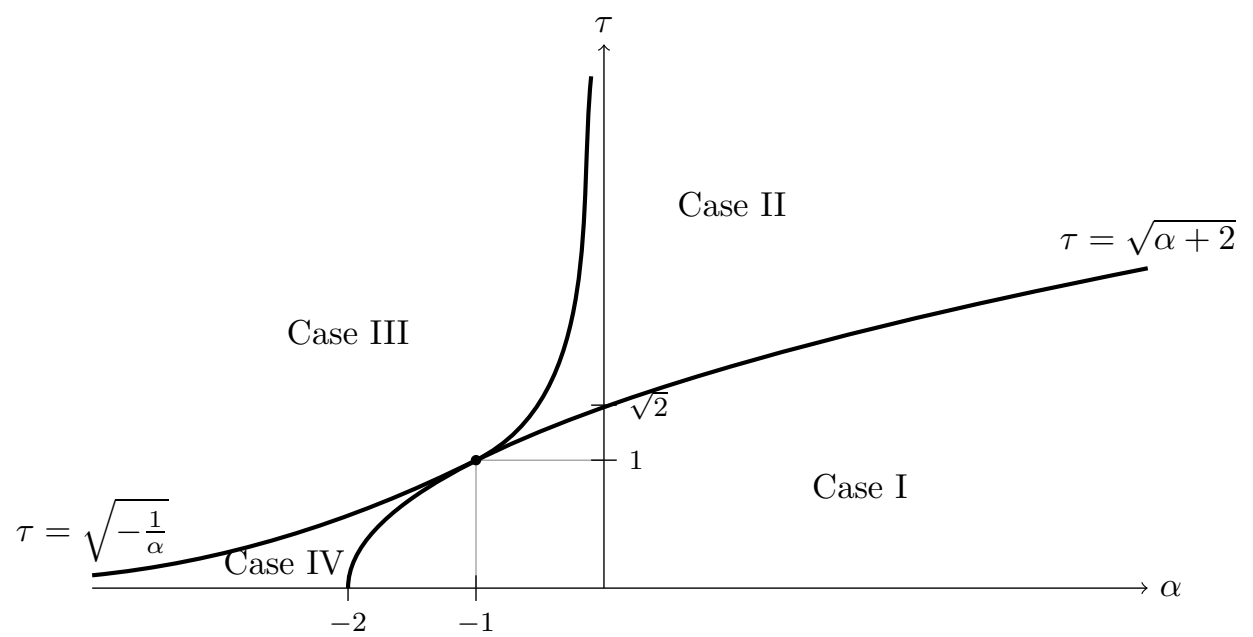

Figure 1.1. Phase diagram in the $\alpha-\tau$ plane for the case $V(x)=$ $\frac{1}{2} x^{2}$ : the curves $\tau=\sqrt{\alpha+2}$ and $\tau=\sqrt{-1 / \alpha}$ separate the phase diagram into four regions. The four regions correspond to the cases: Case I: $N=1, c_{2}>0$ and $c_{3}=0$, Case II: $N=2, c_{2}>0$ and $c_{3}=0$, Case III: $N=2, c_{2}=0$ and $c_{3}>0$, and Case IV: $N=1, c_{2}>0$ and $c_{3}>0$.

Singular exterior point for $\mu_{1}$ : Equality holds in the variational inequality in (1.23) at a point $x \in \mathbb{R} \backslash S\left(\mu_{1}\right)$.

The measures $\sigma_{2}-\mu_{2}$ and $\mu_{3}$ cannot have singular endpoints, singular exterior points, or singular interior points, except at 0 . Singular interior points of these measures at 0 are as follows.

Singular interior point for $\sigma_{2}-\mu_{2}$ : The density of $\sigma_{2}-\mu_{2}$ vanishes at $0 \in S\left(\sigma_{2}-\mu_{2}\right)$.

Singular interior point for $\mu_{3}$ : The density of $\mu_{3}$ vanishes at $0 \in S\left(\mu_{3}\right)$.

While there is great interest in the singular cases we restrict the analysis in this paper to the regular cases, for which we give the following precise definition.

Definition 1.3. The triplet $(V, W, \tau)$ is regular if the supports of the minimizers from the vector equilibrium problem satisfy

$$
S\left(\mu_{1}\right) \cap S\left(\sigma_{2}-\mu_{2}\right)=\emptyset \quad \text { and } \quad S\left(\mu_{3}\right) \cap S\left(\sigma_{2}-\mu_{2}\right)=\emptyset
$$

and if in addition, the measure $\mu_{1}$ has no singular interior points, singular endpoints, or singular exterior points, and the measures $\sigma_{2}-\mu_{2}$ and $\mu_{3}$ do not have a singular interior point at 0 .

The condition (1.32) may be reformulated as

$$
c_{2}=0 \quad \Longrightarrow \quad 0 \notin S\left(\mu_{1}\right) \cup S\left(\mu_{3}\right) .
$$

\subsection{Limiting mean eigenvalue distribution}

The measure $\mu_{1}$ is the limiting mean eigenvalue distribution of the matrix $M_{1}$ in the two matrix model as $n \rightarrow \infty$. In this paper we prove this only for regular 
cases. To prove it for singular cases, one would have to analyze the nature of the singular behavior which is beyond the scope of what we want to do in this paper.

THEOREM 1.4. Suppose $(V, W, \tau)$ is regular. Let $\mu_{1}$ be the first component of the minimizer $\left(\mu_{1}, \mu_{2}, \mu_{3}\right)$ of the vector equilibrium problem. Then $\mu_{1}$ is the limiting mean distribution of the eigenvalues of $M_{1}$ as $n \rightarrow \infty$ with $n \equiv 0(\bmod 3)$.

We recall that the eigenvalues of $M_{1}$ after averaging over $M_{2}$ are a determinantal point process on $\mathbb{R}$ with a kernel $K_{11}^{(n)}$ as given in (1.5). The statement of Theorem 1.4 comes down to the statement that

$$
\lim _{n \rightarrow \infty} \frac{1}{n} K_{11}^{(n)}(x, x)=\rho_{1}(x), \quad x \in \mathbb{R}
$$

where $\rho_{1}$ is the density of the measure $\mu_{1}$.

The restriction to $n \equiv 0(\bmod 3)$ is for convenience only, since it simplifies the expressions in the steepest descent analysis of the Riemann-Hilbert problem that we are going to do.

The existence of the limiting mean eigenvalue distribution was proved by Guionnet [57. in much more general context. She characterized the minimizer by a completely different variational problem, and also connects it with a large deviation principle. It would be very interesting to see the connection with our vector equilibrium problem. A related question would be to ask if it is possible to establish a large deviation principle with the energy functional (1.12) as a good rate function.

We are going to prove (1.33) by applying the Deift-Zhou steepest descent analysis to the Riemann-Hilbert problem (1.36) below. Without too much extra effort we can also obtain the usual universal local scaling limits that are typical for unitary random matrix ensembles. Namely, if $\rho_{1}\left(x^{*}\right)>0$ then the scaling limit is the sine kernel

$$
\lim _{n \rightarrow \infty} \frac{1}{n \rho_{1}\left(x^{*}\right)} K_{11}^{(n)}\left(x^{*}+\frac{x}{n \rho_{1}\left(x^{*}\right)}, x^{*}+\frac{y}{n \rho_{1}\left(x^{*}\right)}\right)=\frac{\sin \pi(x-y)}{\pi(x-y)},
$$

while if $x^{*} \in\left\{a_{1}, b_{1}, \ldots, a_{N}, b_{N}\right\}$ is an end point of $S\left(\mu_{1}\right)$ then the scaling is the Airy kernel, i.e., for some $c>0$, we have

$$
\lim _{n \rightarrow \infty} \frac{1}{(c n)^{2 / 3}} K_{11}^{(n)}\left(x^{*} \pm \frac{x}{(c n)^{2 / 3}}, x^{*} \pm \frac{y}{(c n)^{2 / 3}}\right)=\frac{\operatorname{Ai}(x) \operatorname{Ai}^{\prime}(y)-\operatorname{Ai}^{\prime}(x) \operatorname{Ai}(y)}{x-y}
$$

with + if $x^{*}=b_{j}$ and - if $x^{*}=a_{j}$ for some $j=1, \ldots, N$. Recall that we are in the regular case so that the density of $\rho_{1}$ vanishes as a square root at $x^{*}$. The proofs of these local scaling limits will be omitted here, as they are very similar to the proofs in 45 .

\subsection{About the proof of Theorem 1.4}

The first step in the proof of Theorem 1.4 is the setup of the Riemann-Hilbert $(\mathrm{RH})$ problem for biorthogonal polynomial $p_{n, n}$ and its connection with the correlation kernel $K_{11}^{(n)}$. We use the RH problem of 67. which we briefly recall.

The $\mathrm{RH}$ problem of [67] is based on the observation that the polynomial $p_{n, n}$ that is characterized by the biorthogonality conditions (1.4) can alternatively be characterized by the conditions (we assume $W$ is quartic and $n$ is a multiple of three)

$$
\int_{-\infty}^{\infty} p_{n, n}(x) x^{k} w_{j, n}(x) d x=0, \quad k=0, \ldots, n / 3-1, j=0,1,2
$$


which involves three varying (i.e., $n$-dependent) weight functions

$$
w_{j, n}(x)=e^{-n V(x)} \int_{-\infty}^{\infty} y^{j} e^{-n(W(y)-\tau x y)} d y, \quad j=0,1,2 .
$$

The conditions (1.34) are known as multiple orthogonality conditions of type II, see e.g. [4, 64, 77, 83 .

A RH problem for multiple orthogonal polynomials was given by Van Assche, Geronimo and Kuijlaars in [84 as an extension of the well-known RH problem for orthogonal polynomials of Fokas, Its, and Kitaev [55. For the multiple orthogonality (1.34) the $\mathrm{RH}$ problem is of size $4 \times 4$ and it asks for $Y: \mathbb{C} \backslash \mathbb{R} \rightarrow \mathbb{C}^{4 \times 4}$ satisfying

$$
\left\{\begin{array}{l}
Y \text { is analytic in } \mathbb{C} \backslash \mathbb{R}, \\
Y_{+}(x)=Y_{-}(x)\left(\begin{array}{cccc}
1 & w_{0, n}(x) & w_{1, n}(x) & w_{2, n}(x) \\
0 & 1 & 0 & 0 \\
0 & 0 & 1 & 0 \\
0 & 0 & 0 & 0
\end{array}\right), \quad x \in \mathbb{R}, \\
Y(z)=(I+\mathcal{O}(1 / z))\left(\begin{array}{cccc}
z^{n} & 0 & 0 & 0 \\
0 & z^{-n / 3} & 0 & 0 \\
0 & 0 & z^{-n / 3} & 0 \\
0 & 0 & 0 & z^{-n / 3}
\end{array}\right), \quad z \rightarrow \infty .
\end{array}\right.
$$

The RH problem has a unique solution. The first row of $Y$ is given in terms of the biorthogonal polynomial $p_{n, n}$ as follows

$$
Y_{1,1}(z)=p_{n, n}(z), \quad Y_{1, j+2}(z)=\frac{1}{2 \pi i} \int_{-\infty}^{\infty} \frac{p_{n, n}(x) w_{j, n}(x)}{x-z} d x, \quad j=0,1,2,
$$

and the other rows are built out of certain polynomials of degree $n-1$ in a similar way, see 67, 84 for details.

Multiple orthogonal polynomials have a Christoffel-Darboux formula [29] which implies that the correlation kernel (1.5) can be rewritten in the integrable form

$$
\frac{f_{1}(x) g_{1}(y)+f_{2}(x) g_{2}(y)+f_{3}(x) g_{3}(y)+f_{4}(x) g_{4}(y)}{x-y}
$$

for certain functions $f_{j}, g_{j}$, for $j=1, \ldots, 4$, and in fact it has the following representation

$$
\begin{aligned}
& K_{11}^{(n)}(x, y) \\
& \quad=\frac{1}{2 \pi i(x-y)}\left(\begin{array}{llll}
0 & w_{0, n}(y) & w_{1, n}(y) & \left.w_{2, n}(y)\right) Y_{+}^{-1}(y) Y_{+}(x) \\
& \\
0 \\
0
\end{array}\right),
\end{aligned}
$$

for $x, y \in \mathbb{R}$, in terms of the solution $Y$ of the $\mathrm{RH}$ problem (1.36), see $\mathbf{2 9}$.

The proof of Theorem 1.4 is an involved and lengthy steepest descent analysis of the $\mathrm{RH}$ problem (1.36) in which the vector equilibrium problem is used in an essential way. This is similar to 45 , which deals with the case $\alpha=0$. Certain complications arise because the formulas for the external field and the constraint in the vector equilibrium problem are less explicit as in the case $\alpha=0$. This not only complicates the analysis of the vector equilibrium problem in chapters 2 and 3 . 
but it will continue to play a role via the functions $\theta_{j}$ defined in Section 2.2 and $\lambda_{j}$ defined in Section 4.4 throughout the paper.

We also note that the analysis in [45] was restricted to the one-cut case, which leads to an underlying Riemann surface of genus 0 . This restriction was removed in [76. The problem in the higher genus case is in the construction of the global parametrix. In chapter 8 we give a self-contained account that is based on the ideas developed in [76 and [68, which we think is of independent interest.

We also wish to stress that in Case IV the Riemann surface always has genus $\geq 1$, even if $S\left(\mu_{1}\right)$ consists of one interval, see (4.16) below. This phenomenon did not appear for $\alpha=0$.

\subsection{Singular cases}

Although we do not treat the singular cases in this paper we wish to make some comments about the possible critical behaviors that we see in the two matrix model with the quartic potential $W(y)=\frac{1}{4} y^{4}+\frac{\alpha}{2} y^{2}$.

As already discussed in Section 1.5 the singular behavior is associated with either a singular behavior in the measures $\mu_{1}, \sigma_{2}-\mu_{2}$, or $\mu_{3}$, or a singular behavior in the supports. The singular behavior in the measure $\mu_{1}$ also appears in the one matrix model that is described by orthogonal polynomials. It is known that the critical behavior at a singular interior point where the density vanishes quadratically is described by the Hastings-McLeod solution of the Painlevé II equation, see 19, 27, 80. This Painlevé II transition is the canonical mechanism by which a gap opens up in the support in the one matrix model.

The critical behavior at a singular endpoint where the density vanishes with exponent $5 / 2$ is described by a special solution of the Painlevé $\mathrm{I}_{2}$ equation (the second member of the Painlevé I hierarchy), see 28. The critical behavior at a singular exterior point is described by Hermite functions [16, 26, 75] and this describes an opening of a new band of eigenvalues (birth of a cut).

We see these critical behaviors also in the two matrix model with an even quartic $W$. In particular, the opening of a gap at 0 in the support of $\mu_{1}$ is a Painlevé II transition. In our classification of regular cases, this is a transition from Case I to Case II, or a transition from Case IV to Case V. In the phase diagram of Figure 1.1 for $V(x)=\frac{1}{2} x^{2}$, this transition is on the part of the parabola $\tau=\sqrt{\alpha+2}$, with $\alpha>-1$.

A Painlevé II transition also appears when either $\sigma_{2}-\mu_{2}$ or $\mu_{3}$ has a density that vanishes quadratically at 0 . Then 0 is a singular interior point and again a gap can open but now in the support of the measures "that are on the other sheets" and have no direct probabilistic meaning. If the density of $\sigma_{2}-\mu_{2}$ vanishes at 0 then the transition is from Case III to Case V. If the density of $\mu_{3}$ vanishes at 0 then the transition is from Case I to Case IV or from Case II to Case V. In the phase diagram of Figure 1.1 the transition from Case I to Case IV takes place on the part of the parabola $\tau=\sqrt{\alpha+2}$, with $-2<\alpha<-1$.

The cases of singular supports represent critical phenomena that do not appear in the one matrix model. What we called Singular Supports I in Section 1.5 corresponds to a transition from Case III to Case IV. This is a transition when the gap around 0 in the support of $S\left(\mu_{1}\right)$ closes and simultaneously the gap in the support of $S\left(\sigma_{2}-\mu_{2}\right)$ opens up (or vice versa). On the level of the Riemann surface it means that the two branch points on the real line that are the endpoints of the 
gap in $S\left(\mu_{1}\right)$ come together at 0 , and then split again to become a pair of complex conjugate branch points. These branch points are then on the imaginary axis and are the endpoints $\pm i c_{2}$ of $S\left(\sigma_{2}-\mu_{2}\right)$. A transition of this type does not change the genus of the Riemann surface.

This type of transition was observed first in the context of random matrices with external source and non-intersecting Brownian motions, see [3, 21, 25, 82, where it was described in terms of Pearcey integrals. The Pearcey transition is a second mechanism by which a gap in the support may open up (or close). As it involves three sheets of the Riemann surface it cannot take place in the one matrix model which is connected to a two-sheeted Riemann surface.

The case of Singular Supports II gives a transition from Case II to Case III. This is a situation where the gap in the support of $\sigma_{2}-\mu_{2}$ closes and simultaneously the gap in $S\left(\mu_{3}\right)$ opens. This also typically corresponds to a Pearcey transition, but it does not involve the first sheet of the Riemann surface, which means that this transition is not visible in the eigenvalue distribution of the random matrix. In the phase diagram of Figure 1.1 the Pearcey transitions are on the curve $\tau=\sqrt{-1 / \alpha}$, $\alpha \neq-1$.

The case of Singular Supports III represents a new critical phenomenon. Here the supports of all three measures $\mu_{1}, \sigma_{2}-\mu_{2}$ and $\mu_{3}$ are closed at 0 . In Figure 1.1 this is the case at the multi-critical point $\alpha=-1$ and $\tau=1$ where the Painlevé transitions and Pearcey transitions come together. One may approach the multicritical point from the Case III region, where there is a gap around 0 in the supports of both $\mu_{1}$ and $\mu_{3}$, while the support of $\sigma_{2}-\mu_{2}$ is the full imaginary axis. At the multi-critical point the supports of $\mu_{1}$ and $\mu_{3}$ close simultaneously, while also the support of $\sigma_{2}-\mu_{2}$ opens up, which results in a transition from Case III to Case I.

The case of Singular Supports III shows certain similarities with a critical case of non-intersecting Brownian motions (or random walks) with two starting and two ending points. By fine-tuning the starting and ending points one may create a situation where two groups of non-intersecting Brownian motions fill out two ellipses which are tangent to each other at one point. This so-called tacnode singularity was very recently in a number of papers $[\mathbf{1}, \mathbf{3 8}, 6 \mathbf{6 0}$.

In work in progress of M. Duits and D. Geudens it is shown that the local eigenvalue behavior around 0 in the case of Singular Supports III is indeed closely related to the tacnode behavior for non-intersecting Brownian motions, although it is probably not the same. Indeed, the model Riemann-Hilbert problem that was used in 38 to construct the local parametrix at the tacnode can also be used for the local analysis at the multi-critical point $\alpha=-1$ and $\tau=1$. However, the final expressions for the limiting correlation kernels in terms of the model RiemannHilbert problem are different in the two cases. 


\section{CHAPTER 2}

\section{Preliminaries and the Proof of Lemma 1.2}

Before coming to the proof of Theorem 1.1 we study the equation (1.14) in more detail. This equation will also play a role in the proof of Theorem 1.4, where in the first step of the steepest descent analysis, we will use functions defined by integrals

$$
\int_{\Gamma} e^{-n(W(s)-\tau z s)} d s, \quad W(s)=\frac{1}{4} s^{4}+\frac{\alpha}{2} s^{2}
$$

where $\Gamma$ is an unbounded contour in the complex $s$-plane.

\subsection{Saddle point equation and functions $s_{j}$}

The large $n$ asymptotics of the integrals (2.1) is determined by the solutions of the saddle point equation $W^{\prime}(s)-\tau z=0$, that is

$$
s^{3}+\alpha s=\tau z .
$$

In (1.14) we considered this equation for $z=x \in \mathbb{R}$. We defined a solution $s_{1}(x)$ for every $x \in \mathbb{R}$, and for $\alpha<0$ and $|x|<x^{*}(\alpha)$ we also defined $s_{2}(x)$ and $s_{3}(x)$.

We define solution $s_{1}(z), s_{2}(z)$ and $s_{3}(z)$ of (2.2) for complex $z$ as follows. We distinguish between the two cases $\alpha>0$ and $\alpha<0$.

Case $\alpha>0$. In case $\alpha>0$ the saddle point equation (2.2) has branch points $\pm i y^{*}(\alpha) \in i \mathbb{R}$ where $y^{*}(\alpha)$ is given by (1.21). The Riemann surface $\mathcal{S}$ for the equation (2.2) then has three sheets that we choose as follows

$$
\left\{\begin{array}{l}
\mathcal{S}_{1}=\mathbb{C} \backslash\left(\left(-i \infty,-i y^{*}(\alpha)\right] \cup\left[i y^{*}(\alpha), i \infty\right)\right), \\
\mathcal{S}_{2}=\mathbb{C} \backslash\left(\mathbb{R} \cup\left(-i \infty,-i y^{*}(\alpha)\right] \cup\left[i y^{*}(\alpha), i \infty\right)\right), \\
\mathcal{S}_{3}=\mathbb{C} \backslash \mathbb{R} .
\end{array}\right.
$$

We already defined $s_{1}(x)$ for $x \in \mathbb{R}$ as the unique real saddle point. This function has an analytic continuation to $\mathcal{S}_{1}$ that we also denote by $s_{1}$. Then $s_{2}$ and $s_{3}$ are defined by analytic continuation onto $\mathcal{S}_{2}$ and $\mathcal{S}_{3}$, respectively.

Case $\alpha<0$. In case $\alpha<0$ the saddle point equation (2.2) has two real branch points $\pm x^{*}(\alpha)$ with $x^{*}(\alpha)$ given by (1.16). The three sheets of the Riemann surface $\mathcal{S}$ for the equation (2.2) are now chosen as follows

$$
\left\{\begin{array}{l}
\mathcal{S}_{1}=\mathbb{C} \backslash i \mathbb{R}, \\
\mathcal{S}_{2}=\mathbb{C} \backslash\left(\left(-\infty,-x^{*}(\alpha)\right] \cup\left[x^{*}(\alpha), \infty\right) \cup i \mathbb{R}\right), \\
\mathcal{S}_{3}=\mathbb{C} \backslash\left(\left(-\infty,-x^{*}(\alpha)\right] \cup\left[x^{*}(\alpha), \infty\right)\right) .
\end{array}\right.
$$

In case $\alpha<0$, we have that $s_{1}(x)$ is defined for $x \in \mathbb{R} \backslash\{0\}$. It is the real saddle point for which $W(s)-\tau x s$ is minimal. The function $s_{1}$ has an analytic continuation to $\mathcal{S}_{1}$ that we also denote by $s_{1}$. Then $s_{2}$ and $s_{3}$ are defined by 
analytic continuation onto $\mathcal{S}_{2}$ and $\mathcal{S}_{3}$, respectively. It is a straightforward check that for $x \in\left(-x^{*}(\alpha), x^{*}(\alpha)\right)$ this definition of $s_{2}(x)$ and $s_{3}(x)$ coincides with the one earlier given.

LEMma 2.1. The functions $s_{j}$ are have the symmetries

$$
s_{j}(-z)=-s_{j}(z), \quad s_{j}(\bar{z})=\overline{s_{j}(z)}, \quad j=1,2,3 .
$$

In addition we have that

$$
\operatorname{Re} s_{1}(z)>0 \quad \text { if } \operatorname{Re} z>0 .
$$

ProOf. The symmetries (2.5) are clear.

For $z=x \in \mathbb{R}$ with $x>0$ we have that $s_{1}(x)>0$. Therefore, by continuity, $\operatorname{Re} s_{1}(z)>0$ for $z$ in a neighborhood of the positive real axis. If $\operatorname{Re} s_{1}(z)=0$ for some $z$, so that $s_{1}(z)$ is purely imaginary, then

$$
\tau z=s_{1}(z)^{3}+\alpha s_{1}(z)
$$

is purely imaginary as well. The inequality $\operatorname{Re} s_{1}(z)>0$ therefore extends into the full right half-plane as claimed in (2.6).

From the lemma it follows that in both cases the constraint $\sigma_{2}$, see (1.19), is given by

$$
\begin{aligned}
\frac{d \sigma_{2}(z)}{d z} & =\frac{\tau}{\pi i} \operatorname{Re} s_{1,-}(z) \\
& =\frac{\tau}{2 \pi i}\left(s_{1,-}(z)-s_{1,+}(z)\right), \quad z \in i \mathbb{R} .
\end{aligned}
$$

The imaginary axis is oriented upwards, so that $s_{1,-}(z)\left(s_{1,+}(z)\right)$ for $z \in i \mathbb{R}$ denotes the limiting value of $s_{1}$ as we approach $z \in i \mathbb{R}$ from the right (left) half-plane.

\subsection{Values at the saddles and functions $\theta_{j}$}

We define

$$
\theta_{j}(z)=-W\left(s_{j}(z)\right)+\tau z s_{j}(z), \quad j=1,2,3
$$

as the value of $-(W(s)-\tau z s)$ at the saddle $s=s_{j}(z)$. Note that

$$
\theta_{j}^{\prime}(z)=\left(-W^{\prime}\left(s_{j}(z)\right)+\tau z\right) s_{j}^{\prime}(z)+\tau s_{j}(z)=\tau s_{j}(z)
$$

so that, up to a factor $\tau, \theta_{j}$ is a primitive function of $s_{j}$.

Then $\theta_{j}$ is defined and analytic on $\mathcal{S}_{j}$, see (2.3) and (2.4), and

$$
\begin{cases}\theta_{1, \pm}=\theta_{2, \mp} & \text { on }\left(-i \infty,-i y^{*}(\alpha)\right] \cup\left[i y^{*}(\alpha), i \infty\right), \\ \theta_{2, \pm}=\theta_{3, \mp} & \text { on }\left(-\infty,-x^{*}(\alpha)\right] \cup\left[x^{*}(\alpha), \infty\right) .\end{cases}
$$

Recall from (1.16) and (1.21) that we have put $x^{*}(\alpha)=0$ if $\alpha>0$ and $y^{*}(\alpha)=0$ if $\alpha<0$, so that we can treat the two cases simultaneously in (2.10).

The jumps for the $\theta_{j}$ functions from (2.10), are taken together in terms of the jumps of the diagonal matrix

$$
\Theta(z)=\left(\begin{array}{ccc}
\theta_{1}(z) & 0 & 0 \\
0 & \theta_{2}(z) & 0 \\
0 & 0 & \theta_{3}(z)
\end{array}\right), \quad z \in \mathbb{C} \backslash(\mathbb{R} \cup i \mathbb{R})
$$

as follows. 
Corollary 2.2. For $x \in \mathbb{R}$ we have

$$
\begin{cases}\Theta_{+}(x)=\Theta_{-}(x), & |x|<x^{*}(\alpha), \\
\Theta_{+}(x)=\left(\begin{array}{lll}
1 & 0 & 0 \\
0 & 0 & 1 \\
0 & 1 & 0
\end{array}\right) \Theta_{-}(x)\left(\begin{array}{lll}
1 & 0 & 0 \\
0 & 0 & 1 \\
0 & 1 & 0
\end{array}\right), & |x|>x^{*}(\alpha) .\end{cases}
$$

For $z=i y \in i \mathbb{R}$ we have

$$
\begin{cases}\Theta_{+}(z)=\Theta_{-}(z), & |y|<y^{*}(\alpha), \\
\Theta_{+}(z)=\left(\begin{array}{lll}
0 & 1 & 0 \\
1 & 0 & 0 \\
0 & 0 & 1
\end{array}\right) \Theta_{-}(z)\left(\begin{array}{lll}
0 & 1 & 0 \\
1 & 0 & 0 \\
0 & 0 & 1
\end{array}\right), & |y|>y^{*}(\alpha) .\end{cases}
$$

Also note that by (1.13), the definition of $s_{1}$ and (2.8), we have

$$
V_{1}(x)=V(x)-\theta_{1}(x), \quad x \in \mathbb{R},
$$

and by (1.15)-(1.17), the definition of $s_{2}$ and $s_{3}$, and (2.8)

$$
V_{3}(x)=\left\{\begin{array}{cl}
\theta_{2}(x)-\theta_{3}(x), & \text { for } x \in\left(-x^{*}(\alpha), x^{*}(\alpha)\right), \\
0, & \text { elsewhere }
\end{array}\right.
$$

\subsection{Large $z$ asymptotics}

In what follows we will need the behavior of $s_{j}(z)$ and $\theta_{j}(z)$ as $z \rightarrow \infty$.

Throughout the paper we define fractional exponents with a branch cut along the negative real axis. We use $I, I I, I I I$ and $I V$ to denote the four quadrants of the complex $z$-plane. We also put

$$
\omega=e^{2 \pi i / 3} .
$$

We state the following lemma without proof. It follows easily from the saddle point equation (2.2).

Lemma 2.3. We have as $z \rightarrow \infty$

$$
\begin{aligned}
& s_{1}(z)= \begin{cases}(\tau z)^{1 / 3}-\frac{\alpha}{3}(\tau z)^{-1 / 3}+\frac{\alpha^{3}}{81}(\tau z)^{-5 / 3}+\mathcal{O}\left(z^{-7 / 3}\right), & \text { in } I \cup I V, \\
\omega(\tau z)^{1 / 3}-\frac{\alpha}{3} \omega^{2}(\tau z)^{-1 / 3}+\frac{\alpha^{3}}{81} \omega(\tau z)^{-5 / 3}+\mathcal{O}\left(z^{-7 / 3}\right), & \text { in } I I, \\
\omega^{2}(\tau z)^{1 / 3}-\frac{\alpha}{3} \omega(\tau z)^{-1 / 3}+\frac{\alpha^{3}}{81} \omega^{2}(\tau z)^{-5 / 3}+\mathcal{O}\left(z^{-7 / 3}\right), & \text { in } I I I,\end{cases} \\
& s_{2}(z)= \begin{cases}\omega(\tau z)^{1 / 3}-\frac{\alpha}{3} \omega^{2}(\tau z)^{-1 / 3}+\frac{\alpha^{3}}{81} \omega(\tau z)^{-5 / 3}+\mathcal{O}\left(z^{-7 / 3}\right), & \text { in } I \\
(\tau z)^{1 / 3}-\frac{\alpha}{3}(\tau z)^{-1 / 3}+\frac{\alpha^{3}}{81}(\tau z)^{-5 / 3}+\mathcal{O}\left(z^{-7 / 3}\right), & \text { in } I I \cup I I I, \\
\omega^{2}(\tau z)^{1 / 3}-\frac{\alpha}{3} \omega(\tau z)^{-1 / 3}+\frac{\alpha^{3}}{81} \omega^{2}(\tau z)^{-5 / 3}+\mathcal{O}\left(z^{-7 / 3}\right), & \text { in } I V,\end{cases} \\
& s_{3}(z)= \begin{cases}\omega^{2}(\tau z)^{1 / 3}-\frac{\alpha}{3} \omega(\tau z)^{-1 / 3}+\frac{\alpha^{3}}{81} \omega^{2}(\tau z)^{-5 / 3}+\mathcal{O}\left(z^{-7 / 3}\right), & \text { in } I \cup I I \\
\omega(\tau z)^{1 / 3}-\frac{\alpha}{3} \omega^{2}(\tau z)^{-1 / 3}+\frac{\alpha^{3}}{81} \omega(\tau z)^{-5 / 3}+\mathcal{O}\left(z^{-7 / 3}\right), & \text { in } I I I \cup I V .\end{cases}
\end{aligned}
$$

We have a similar result for the asymptotics of $\theta_{j}$. Note that the following asymptotic behaviors are consistent with the property that $\theta_{j}^{\prime}=\tau s_{j}$, see (2.9).

LEMMA 2.4. We have as $z \rightarrow \infty$

$$
\theta_{1}(z)= \begin{cases}\frac{3}{4}(\tau z)^{4 / 3}-\frac{\alpha}{2}(\tau z)^{2 / 3}+\frac{\alpha^{2}}{6}-\frac{\alpha^{3}}{54}(\tau z)^{-2 / 3}+\mathcal{O}\left(z^{-4 / 3}\right), & \text { in } I \cup I V \\ \frac{3}{4} \omega(\tau z)^{4 / 3}-\frac{\alpha}{2} \omega^{2}(\tau z)^{2 / 3}+\frac{\alpha^{2}}{6}-\frac{\alpha^{3}}{54} \omega(\tau z)^{-2 / 3}+\mathcal{O}\left(z^{-4 / 3}\right), & \text { in } I I, \\ \frac{3}{4} \omega^{2}(\tau z)^{4 / 3}-\frac{\alpha}{2} \omega(\tau z)^{2 / 3}+\frac{\alpha^{2}}{6}-\frac{\alpha^{3}}{54} \omega^{2}(\tau z)^{-2 / 3}+\mathcal{O}\left(z^{-4 / 3}\right), & \text { in } I I I,\end{cases}
$$




$$
\begin{aligned}
& \theta_{2}(z)= \begin{cases}\frac{3}{4} \omega(\tau z)^{4 / 3}-\frac{\alpha}{2} \omega^{2}(\tau z)^{2 / 3}+\frac{\alpha^{2}}{6}-\frac{\alpha^{3}}{54} \omega(\tau z)^{-2 / 3}+\mathcal{O}\left(z^{-4 / 3}\right), & \text { in } I \\
\frac{3}{4}(\tau z)^{4 / 3}-\frac{\alpha}{2}(\tau z)^{2 / 3}+\frac{\alpha^{2}}{6}-\frac{\alpha^{3}}{54}(\tau z)^{-2 / 3}+\mathcal{O}\left(z^{-4 / 3}\right), & \text { in } I I \cup I I I, \\
\frac{3}{4} \omega^{2}(\tau z)^{4 / 3}-\frac{\alpha}{2} \omega(\tau z)^{2 / 3}+\frac{\alpha^{2}}{6}-\frac{\alpha^{3}}{54} \omega^{2}(\tau z)^{-2 / 3}+\mathcal{O}\left(z^{-4 / 3}\right), & \text { in } I V,\end{cases} \\
& \theta_{3}(z)= \begin{cases}\frac{3}{4} \omega^{2}(\tau z)^{4 / 3}-\frac{\alpha}{2} \omega(\tau z)^{2 / 3}+\frac{\alpha^{2}}{6}-\frac{\alpha^{3}}{54} \omega^{2}(\tau z)^{-2 / 3}+\mathcal{O}\left(z^{-4 / 3}\right), & \text { in } I \cup I I \\
\frac{3}{4} \omega(\tau z)^{4 / 3}-\frac{\alpha}{2} \omega^{2}(\tau z)^{2 / 3}+\frac{\alpha^{2}}{6}-\frac{\alpha^{3}}{54} \omega(\tau z)^{-2 / 3}+\mathcal{O}\left(z^{-4 / 3}\right), & \text { in } I I I \cup I V .\end{cases}
\end{aligned}
$$

\subsection{Two special integrals}

As a final preparation for the proof of Lemma 1.2 we need the evaluation of the following two definite integrals.

LEMMA 2.5. We have for $x>0$

$$
\int_{i \mathbb{R}} \frac{d \sigma_{2}(z)}{(x-z)^{2}}=-\tau s_{1}^{\prime}(x), \quad \text { and } \quad \int_{i \mathbb{R}} \frac{d \sigma_{2}(z)}{x-z^{2}}=\frac{\tau s_{1}(\sqrt{x})}{\sqrt{x}} .
$$

Proof. Because of the formula (2.7) for $\sigma_{2}$ we have

$$
\int_{i \mathbb{R}} \frac{d \sigma_{2}(z)}{(x-z)^{2}}=\frac{\tau}{2 \pi i} \int_{i \mathbb{R}} \frac{s_{1,-}(z)-s_{1,+}(z)}{(x-z)^{2}} d z
$$

Since $s_{1}$ is analytic in $\mathbb{C} \backslash i \mathbb{R}$ and $s_{1}(z)=\mathcal{O}\left(z^{1 / 3}\right)$ as $z \rightarrow \infty$, see Lemma 2.3, we can evaluate the integral using contour integration and residue calculus. It follows that

$$
\frac{\tau}{2 \pi i} \int_{i \mathbb{R}} \frac{s_{1,-}(z)}{(x-z)^{2}} d z=-\tau s_{1}^{\prime}(x), \quad \text { and } \quad \frac{\tau}{2 \pi i} \int_{i \mathbb{R}} \frac{s_{1,+}(z)}{(x-z)^{2}} d z=0,
$$

and the first integral in (2.16) is proved.

The second integral follows by a similar calculation, where we also use the fact that $s_{1}$ is an odd function.

\subsection{Proof of Lemma 1.2}

Now we come to the proof of Lemma 1.2

\subsubsection{Proof of part (a).}

Proof. Integrating the first formula in (2.16) two times with respect to $x$, and using the fact that $\theta_{1}^{\prime}=\tau s_{1}$, we find that for some constants $A$ and $B$,

$$
\int_{i \mathbb{R}}(\log |z-x|-\log |z|) d \sigma_{2}(z)=\theta_{1}(x)+A x+B, \quad x>0 .
$$

Thus

$$
\begin{aligned}
V_{1}(x)-U^{\nu_{2}}(x) & =V(x)-\theta_{1}(x)+\int \log |z-x| d \nu_{2}(x) \\
& =V(x)-\int_{i \mathbb{R}}(\log |z-x|-\log |z|) d\left(\sigma_{2}-\nu_{2}\right)(z)-A x-B^{\prime}
\end{aligned}
$$

with a different constant $B^{\prime}=B-\int \log |z| d \nu_{2}(z)$.

Since $0 \notin S\left(\sigma_{2}-\nu_{2}\right)$ there exists a $c>0$ such that $S\left(\sigma_{2}-\nu_{2}\right) \subset i \mathbb{R} \backslash(-i c, i c)$ and so the integral in the right-hand side of (2.17) defines a real analytic function of $x$. Then (2.17) proves that $V_{1}-U^{\nu_{2}}$ is real analytic on $\mathbb{R}$, since $V$ is a polynomial. 


\subsubsection{Proof of part (b).}

Proof. We have by (2.7)

$$
\frac{d \sigma_{2}}{|d z|}(i y)=\frac{\tau}{\pi} \operatorname{Re} s_{1,-}(i y)
$$

Since $s_{1}$ is a solution of $s^{3}+\alpha s=\tau z$, we have $\left(3 s_{1}^{2}+\alpha\right) s_{1}^{\prime}=\tau$, so that

$$
\frac{d}{d y} \operatorname{Re} s_{1,-}(i y)=-\tau \operatorname{Im} \frac{1}{3 s_{1,-}^{2}(i y)+\alpha} .
$$

For $y>0$ we have $\operatorname{Re} s_{1,-}(i y) \geq 0$ by (2.6). We also have $\operatorname{Im} s_{1,-}(i y)>0$, so that $\operatorname{Im}\left(s_{1,-}^{2}(i y)\right) \geq 0$. This implies $\operatorname{Im}\left(1 /\left(3 s_{1,-}^{2}(i y)+\alpha\right)\right) \leq 0$ and so indeed by (2.18)

$$
\frac{d}{d y} \operatorname{Re} s_{1,-}(i y) \geq 0, \quad y>0
$$

which proves part (b) of the lemma.

\subsubsection{Proof of part (c).}

Proof. Let $\alpha<0$ and let $\nu_{2}$ be as in Lemma 1.2 (c). Since $\nu_{2}$ is a measure on $i \mathbb{R}$ we have for $x \in \mathbb{R}, x>0$,

$$
-U^{\nu_{2}}(\sqrt{x})=\frac{1}{2} \int_{i \mathbb{R}} \log \left(x-z^{2}\right) d \nu_{2}(z), \quad x>0 .
$$

Hence

$$
\frac{d^{2}}{d x^{2}}\left(-U^{\nu_{2}}(\sqrt{x})\right)=-\frac{1}{2} \int_{i \mathbb{R}} \frac{1}{\left(x-z^{2}\right)^{2}} d \nu_{2}(z), \quad x>0 .
$$

Since $\nu_{2} \leq \sigma_{2}$ and the integrand is positive for every $z \in i \mathbb{R}$, we have

$$
\begin{aligned}
\frac{d^{2}}{d x^{2}}\left(-U^{\nu_{2}}(\sqrt{x})\right) & >-\frac{1}{2} \int_{i \mathbb{R}} \frac{1}{\left(x-z^{2}\right)^{2}} d \sigma_{2}(z) \\
& =\frac{1}{2} \frac{d}{d x}\left(\int_{i \mathbb{R}} \frac{1}{x-z^{2}} d \sigma_{2}(z)\right) \\
& =\frac{\tau}{2} \frac{d}{d x}\left(\frac{s_{1}(\sqrt{x})}{\sqrt{x}}\right) .
\end{aligned}
$$

where we used the second integral in (2.16).

Since $\theta_{1}^{\prime}=\tau s_{1}$ we see that

$$
\frac{d^{2}}{d x^{2}}\left(-U^{\nu_{2}}(\sqrt{x})\right)>\frac{d^{2}}{d x^{2}}\left(\theta_{1}(\sqrt{x})\right)
$$

Since $V_{3}=\theta_{2}-\theta_{3}$, see (2.14), we have for $0<x<\left(x^{*}(\alpha)\right)^{2}$,

$$
\begin{aligned}
\frac{d^{2}}{d x^{2}}\left(V_{3}(\sqrt{x})-U^{\nu_{2}}(\sqrt{x})\right)>\frac{d^{2}}{d x^{2}}\left(\theta_{2}(\sqrt{x})-\theta_{3}(\sqrt{x})+\theta_{1}(\sqrt{x})\right) & \\
& 0<x \leq\left(x^{*}(\alpha)\right)^{2} .
\end{aligned}
$$

Since $\theta_{1}+\theta_{2}+\theta_{3}=\frac{1}{2} \alpha^{2}$, it now also follows that

$$
\frac{d^{2}}{d x^{2}}\left(V_{3}(\sqrt{x})-U^{\nu_{2}}(\sqrt{x})\right)>-2 \frac{d^{2}}{d x^{2}}\left(\theta_{3}(\sqrt{x})\right), \quad 0<x \leq\left(x^{*}(\alpha)\right)^{2} .
$$


Recall that $s_{3}(z)$ is the solution of $s^{3}+\alpha s=\tau z$ with $s_{3}(0)=0$. Since $\alpha<0$ we have that $s_{3}$ is an odd function which is analytic in a neighborhood of 0 . Inserting the Taylor series

$$
s_{3}(z)=-\sum_{k=0}^{\infty} c_{k} z^{2 k+1}, \quad|z|<x^{*}(\alpha),
$$

with $c_{0}=-\frac{\tau}{\alpha}>0$ into $\left[s_{3}(z)\right]^{3}=-\alpha s_{3}(z)+\tau z$ and comparing coefficients of $z$, it is easy to show inductively that $c_{k}>0$ for every $k$. Since $\theta_{3}^{\prime}(z)=\tau s_{3}(z)$ with $\theta_{3}(0)=0$, we then also have

$$
-\theta_{3}(z)=\tau \sum_{k=0}^{\infty} \frac{c_{k}}{2 k+2} z^{2 k+2}, \quad|z|<x^{*}(\alpha)
$$

and

$$
-2 \frac{d^{2}}{d x^{2}} \theta_{3}(\sqrt{x})=\tau \sum_{k=1}^{\infty} k c_{k} x^{k}>0, \quad 0<x<\left(x^{*}(\alpha)\right)^{2}
$$

since all $c_{k}>0$. The two inequalities (2.19) and (2.20) give the convexity of $V_{3}(\sqrt{x})-U^{\nu_{2}}(\sqrt{x})$, which completes the proof of part (c) of Lemma 1.2 


\section{CHAPTER 3}

\section{Proof of Theorem 1.1}

We basically follow Section 4 of 45 where Theorem 1.1 was proved for the case $\alpha=0$. However, we need more additional results from potential theory.

\subsection{Results from potential theory}

We use a number of results and notions from logarithmic potential theory. The main reference is $\mathbf{7 9}$. Most results in [79] are stated for measures with compact support, while we are also dealing with measures with unbounded support, namely the real line or the imaginary axis. Therefore we need a number of results from [79] in a slightly stronger form that allows for measures with unbounded supports.

The following theorem is known as the principle of domination, and it is stated in [79. Theorem II.3.2] for the case where $\mu$ and $\nu$ have compact supports.

THEOREM 3.1. Suppose $\mu$ and $\nu$ are finite Borel measures on $\mathbb{C}$ with $\int d \nu \leq$ $\int d \mu$. Suppose that $\mu$ has finite logarithmic energy and $S(\mu) \neq \mathbb{C}$. If for some constant $c$ the inequality

$$
U^{\mu}(z) \leq U^{\nu}(z)+c
$$

holds $\mu$-almost everywhere, then it holds for all $z \in \mathbb{C}$.

Proof. Let us first establish Theorem 3.1 under the assumption that $\mu$ has compact support, say $S(\mu) \subset D_{R}=\{z|| z \mid \leq R\}$. Let $\hat{\nu}$ be the balayage of $\nu$ onto $D_{R}$. By the properties of balayage [79], we then have $\int d \hat{\nu}=\int d \nu$ and for certain constant $\ell \geq 0$,

$$
\begin{array}{ll}
U^{\hat{\nu}}(z)=U^{\nu}(z)+\ell, & z \in D_{R}, \\
U^{\hat{\nu}}(z) \leq U^{\nu}(z)+\ell, & z \in \mathbb{C} .
\end{array}
$$

Then if (3.1) holds $\mu$-a.e., we find from the equality in (3.2) and the fact that $S(\mu) \subset D_{R}$ that

$$
U^{\mu}(z) \leq U^{\hat{\nu}}(z)+c-\ell \quad \mu \text {-a.e.. }
$$

Thus by the principle of domination for measures with compact supports, see 79 , Theorem II.3.2], we find $U^{\mu} \leq U^{\hat{\nu}}+c-\ell$ on $\mathbb{C}$, which in view of the inequality in (3.2) leads to $U^{\mu} \leq U^{\nu}+c$ on $\mathbb{C}$, as required.

We next assume that $\mu$ is as in the statement of the theorem. Since $S(\mu) \neq \mathbb{C}$, there is some disk $D\left(z_{0}, r\right)=\left\{z \in \mathbb{C}|| z-z_{0} \mid<r\right\}$ with $r>0$ that is disjoint from $S(\mu)$. By translation and dilation invariance of the statement in Theorem 3.1 we may assume that $D(0,1)$ is disjoint from $S(\mu)$. We may also assume that $U^{\nu}(0)<\infty$. 
Let $\tilde{\mu}$ be the image of $\mu$ under the inversion $z \mapsto 1 / z$. Then $\tilde{\mu}$ has compact support and straightforward calculations shows that

$$
U^{\tilde{\mu}}(z)=U^{\mu}(1 / z)-\log |z| \int d \mu-U^{\mu}(0)
$$

and $I(\tilde{\mu})=I(\mu)$. If $\tilde{\nu}$ is the image of $\nu$ under the inversion $z \mapsto 1 / z$, then we likewise have

$$
U^{\tilde{\nu}}(z)=U^{\nu}(1 / z)-\log |z| \int d \nu-U^{\nu}(0)
$$

Then from (3.1), (3.3) and (3.4), we get

$$
U^{\tilde{\mu}} \leq U^{\tilde{\nu}}-\log |z|\left(\int d \mu-\int d \nu\right)+c-U^{\mu}(0)+U^{\nu}(0), \quad \tilde{\mu} \text { - a.e. }
$$

Thus $U^{\tilde{\mu}} \leq U^{\nu_{1}}+c_{1}$, $\tilde{\mu}$-a.e. where $c_{1}=c-U^{\mu}(0)+U^{\nu}(0)$ and

$$
\nu_{1}=\tilde{\nu}+\left(\int d \mu-\int d \nu\right) \delta_{0}
$$

is a finite positive measure with the same total mass as $\tilde{\mu}$. By the principle of domination for compactly supported measures $\mu$ and arbitrary $\nu$, that we just proved, we find $U^{\tilde{\mu}} \leq U^{\nu_{1}}+c_{1}$ everywhere, which in turn by (3.3) and (3.4) leads to $U^{\mu} \leq U^{\nu}+c$. This proves the theorem.

The following result is stated for compactly supported measures in $\mathbf{7 9}$, Theorem IV.4.5], see also [81 where the result is attributed to de la Vallée Poussin 36 .

THEOREM 3.2. Let $\mu$ and $\nu$ be Borel measures on $\mathbb{C}$ with $\int d \nu \leq \int d \mu, S(\mu) \neq$ $\mathbb{C}$, and finite logarithmic potentials $U^{\mu}$ and $U^{\nu}$. Suppose that for some $c \in \mathbb{R}$, we have

$$
U^{\mu}(z) \leq U^{\nu}(z)+c, \quad z \in S(\mu)
$$

Let

Then

$$
A=\left\{z \in \mathbb{C} \mid U^{\mu}(z)=U^{\nu}(z)+c\right\}
$$

$$
\left.\nu\right|_{A} \leq\left.\mu\right|_{A}
$$

in the sense that $\nu(B) \leq \mu(B)$ for every Borel set $B \subset A$.

Proof. By Theorem 3.1 we obtain from (3.5) that

$$
U^{\mu}(z) \leq U^{\nu}(z)+c, \quad z \in \mathbb{C} .
$$

It is enough to consider bounded Borel sets $B \subset A$. Given such a $B$ we choose $R>0$ such that $|z|<R / 2$ for every $z \in B$. Let $\hat{\mu}$ and $\hat{\nu}$ be the balayages of $\mu$ and $\nu$ onto the closed disk $D_{R}:=\{z \in \mathbb{C}|| z|\leq| R \mid\}$. By the properties of balayage we have, for certain constants $\ell_{1}$ and $\ell_{2}$,

$$
U^{\hat{\mu}}(z)=U^{\mu}(z)+\ell_{1}, \quad U^{\hat{\nu}}(z)=U^{\nu}(z)+\ell_{2}, \quad z \in D_{R} .
$$

It then follows from (3.6) that

$$
U^{\hat{\mu}}(z) \leq U^{\hat{\nu}}(z)+c+\ell_{1}-\ell_{2}, \quad z \in D_{R}
$$

and again by Theorem 3.1 the inequality extends to all of $\mathbb{C}$, since $S(\hat{\mu}) \subset D_{R}$. Equality holds in (3.7) for $z \in D_{R} \cap A$, and so in particular for $z \in B$. 
Then by [79, Theorem IV.4.5], we have that $\hat{\nu}(B) \leq \hat{\mu}(B)$. Then also $\nu(B) \leq$ $\mu(B)$ since $B$ is contained in the interior of $D_{R}$ and on $D_{R}$ the balayage measures $\hat{\mu}$ and $\hat{\nu}$ differ from $\mu$ and $\nu$ only on the boundary $\partial D_{R}=\{z \in \mathbb{C}|| z \mid=R\}$.

We do not know if the condition $S(\mu) \neq \mathbb{C}$ is necessary in Theorems 3.1 and 3.2. The condition is more than sufficient for the purposes of this paper, since we will only be dealing with measures that are supported on either the real line or the imaginary axis.

\subsection{Equilibrium problem for $\nu_{3}$}

Given a measure $\nu_{2} \leq \sigma_{2}$ on $i \mathbb{R}$ with finite logarithmic energy and $\int d \nu_{2}=2 / 3$, the equilibrium problem for $\nu_{3}$ is to minimize

$$
I(\nu)+\int\left(V_{3}(x)-U^{\nu_{2}}(x)\right) d \nu(x)
$$

among all measures $\nu$ on $\mathbb{R}$ with $\int d \nu=1 / 3$. In case $\alpha>0$ we have $V_{3} \equiv 0$ and then we have that the minimizer $\nu_{3}$ of (3.8) is equal to

$$
\nu_{3}=\frac{1}{2} \hat{\nu}_{2}=\frac{1}{2} \operatorname{Bal}\left(\nu_{2}, \mathbb{R}\right)
$$

where $\hat{\nu}_{2}$ denotes the balayage of $\nu_{2}$ onto $\mathbb{R}$. Then $\nu_{3}$ has the density

$$
\frac{d \nu_{3}}{d x}=\frac{1}{\pi} \int_{i \mathbb{R}} \frac{|z|}{x^{2}+|z|^{2}} d \nu_{2}(z)
$$

and the support of $\nu_{3}$ is the full real line. This is similar to what is happening for the case $\alpha=0$ in [45, Section 4.2].

In case $\alpha<0$, the external field $V_{3}$ is positive on the interval $\left(-x^{*}(\alpha), x^{*}(\alpha)\right)$ and zero outside. We use this fact to prove the following inequalities for $\nu_{3}$.

LEMMA 3.3. Let $\nu_{3}$ be the minimizer for (3.8) among measures on $\mathbb{R}$ with $\int d \nu=1 / 3$. Let $c \geq x^{*}(\alpha)$, and let

$$
A_{c}=\mathbb{R} \backslash(-c, c) .
$$

Then we have

$$
\left.\left(\nu_{3}\right)\right|_{A_{c}} \geq\left.\frac{1}{2} \operatorname{Bal}\left(\nu_{2}, \mathbb{R}\right)\right|_{A_{c}} .
$$

and

$$
\left.\left(\nu_{3}\right)\right|_{A_{c}} \leq \frac{1}{2} \operatorname{Bal}\left(\nu_{2}, A_{c}\right) .
$$

PROOF. The variational conditions associated with the minimization problem for (3.8) are

$$
\begin{cases}2 U^{\nu_{3}}(x)+V_{3}(x)-U^{\nu_{2}}(x)=\ell, & x \in S\left(\nu_{3}\right), \\ 2 U^{\nu_{3}}(x)+V_{3}(x)-U^{\nu_{2}}(x) \geq \ell, & x \in \mathbb{R},\end{cases}
$$

where $\ell$ is a constant. Let $\hat{\nu}_{2}=\operatorname{Bal}\left(\nu_{2}, \mathbb{R}\right)$ be the balayage of $\nu_{2}$ onto $\mathbb{R}$. Then $U^{\hat{\nu}_{2}}=U^{\nu_{2}}$ on $\mathbb{R}$, so that it follows from (3.12) that

$$
2 U^{\nu_{3}}(x)=U^{\hat{\nu}_{2}}(x)-V_{3}(x)+\ell, \quad x \in S\left(\nu_{3}\right) .
$$

Since $V_{3}(x) \geq 0$, we conclude that

$$
2 U^{\nu_{3}}(x) \leq U^{\hat{\nu}_{2}}(x)+\ell, \quad x \in S\left(\nu_{3}\right),
$$


By the principle of domination, see Theorem 3.1 (note that the total masses of $2 \nu_{3}$ and $\hat{\nu}_{2}$ are equal), we have that the inequality holds for every $x \in \mathbb{C}$,

$$
2 U^{\nu_{3}}(x) \leq U^{\hat{\nu}_{2}}(x)+\ell, \quad x \in \mathbb{C} .
$$

For $x \in A_{c}$, we have $V_{3}(x)=0$ because of the definition (3.9) with $c \geq x^{*}(\alpha)$. Hence

$$
2 U^{\nu_{3}}(x) \geq U^{\nu_{2}}(x)+\ell=U^{\hat{\nu}_{2}}(x)+\ell, \quad x \in A_{c},
$$

because of the inequality in (3.12). Then by inequality (3.13), we find that equality holds. Thus

$$
A_{c} \subset\left\{x \mid 2 U^{\nu_{3}}(x)=U^{\hat{\nu}_{2}}(x)+\ell\right\},
$$

and the inequality (3.10) follows because of (3.13) and Theorem 3.2 .

The second inequality (3.11) follows in a similar (even simpler) way. Now we redefine $\hat{\nu}_{2}$ as the balayage of $\nu_{2}$ onto $A_{c}$ :

$$
\hat{\nu}_{2}=\operatorname{Bal}\left(\nu_{2}, A_{c}\right) \text {. }
$$

Let $x \in A_{c}$. Then $x \in S\left(\nu_{3}\right)$ because of (3.10), which has already been proved. Then we have, by the property of balayage and (3.12), since $V_{3}(x)=0$ for $x \in A_{c}$,

$$
U^{\hat{\nu}_{2}}(x)=U^{\nu_{2}}(x)=2 U^{\nu_{3}}(x)-\ell, \quad x \in A_{c}=S\left(\hat{\nu}_{2}\right) .
$$

Thus by another application of Theorem 3.2 we find (3.11).

We note that it follows from (3.10) with $c=x^{*}(\alpha)$, that

$$
\left(-\infty,-x^{*}(\alpha)\right] \cup\left[x^{*}(\alpha), \infty\right) \subset S\left(\nu_{3}\right) .
$$

For every $c \geq x^{*}(\alpha)$, we find from (3.11) and the explicit expression for the balayage onto $A_{c}$, that

$$
\frac{d \nu_{3}}{d x} \leq \frac{1}{2 \pi} \frac{|x|}{\sqrt{x^{2}-c^{2}}} \int_{i \mathbb{R}} \frac{\sqrt{c^{2}+|z|}}{x^{2}+|z|^{2}} d \nu_{2}(z), \quad x \in \mathbb{R},|x|>c .
$$

In the case $\alpha<0$ we make use of Lemma 1.2 (c) and Lemma 3.3 to conclude that the support of the minimizer $\nu_{3}$ is of the desired form. This is done in the next lemma.

Proposition 3.4. Let $\nu_{2}$ be a measure on $i \mathbb{R}$ with $\int d \nu_{2}=2 / 3$ and $\nu_{2} \leq \sigma_{2}$. Assume $\nu_{2}$ has finite logarithmic energy. Let $\nu_{3}$ be the minimizer of (3.8) among all measures on $\mathbb{R}$ with total mass $1 / 3$. Then the support of $\nu_{3}$ is of the form

$$
S\left(\nu_{3}\right)=\mathbb{R} \backslash\left(-c_{3}, c_{3}\right)
$$

for some $c_{3} \geq 0$.

If $\alpha<0$ then $c_{3}<x^{*}(\alpha)$, and if $c_{3}>0$ then the density of $\nu_{3}$ vanishes as a square root at $\pm c_{3}$.

Proof. If $\alpha \geq 0$, then $S\left(\nu_{3}\right)=\mathbb{R}$ and we have (3.16) with $c_{3}=0$.

For $\alpha<0$, we use the fact that the external field $V_{3}(x)-U^{\nu_{2}}(x)$ is even. So it follows from [79, Theorem IV.1.10 (f)] that $d \nu_{3}(x)=d \tilde{\nu}_{3}\left(x^{2}\right) / 2$ defines a measure $\tilde{\nu}_{3}$ which is the minimizer of the functional

$$
I(\nu)+2 \int\left(V_{3}(\sqrt{x})-U^{\nu_{2}}(\sqrt{x})\right) d \nu(x)
$$

among measures $\nu$ on $[0, \infty)$ with $\int d \nu=1 / 3$. Note that

$$
S\left(\tilde{\nu}_{3}\right)=\left\{x^{2} \mid x \in S\left(\nu_{3}\right)\right\} \text {. }
$$


The external field $V_{3}(\sqrt{x})-U^{\nu_{2}}(\sqrt{x})$ is convex on $\left[0,\left(x^{*}(\alpha)\right)^{2}\right]$ by Lemma $1.2(\mathrm{c})$, which implies by [79, Theorem IV.1.10 (b)] that $S\left(\tilde{\nu}_{3}\right) \cap\left[0,\left(x^{*}(\alpha)\right)^{2}\right]$ is an interval.

By (3.14) we already know that $x^{*}(\alpha) \in S\left(\nu_{3}\right)$. Then $x^{*}(\alpha)^{2} \in S\left(\tilde{\nu}_{3}\right)$, and it follows that

$$
S\left(\tilde{\nu}_{3}\right) \cap\left[0, x^{*}(\alpha)^{2}\right]=\left[c_{3}^{2}, x^{*}(\alpha)^{2}\right], \quad \text { for some } c_{3} \in\left[0, x^{*}(\alpha)\right] .
$$

Combining this with (3.14), 3.17), we find (3.16).

Now suppose $c_{3}=x^{*}(\alpha)>0$. The minimizer $\nu_{3}$ is characterized by the variational conditions

$$
\begin{cases}2 U^{\nu_{3}}(x)=U^{\nu_{2}}(x)-V_{3}(x), & x \in S\left(\nu_{3}\right)=\left(-\infty,-c_{3}\right] \cup\left[c_{3}, \infty\right), \\ 2 U^{\nu_{3}}(x)>U^{\nu_{2}}(x)-V_{3}(x), & x \in \mathbb{R} \backslash S\left(\nu_{3}\right),\end{cases}
$$

where the inequality on $\mathbb{R} \backslash S\left(\nu_{3}\right)$ is indeed strict due to the convexity of $V_{3}(\sqrt{x})$ $U^{\nu_{2}}(\sqrt{x})$. Since $c_{3}=x^{*}(\alpha)$, we have that $2 U^{\nu_{3}}=U^{\nu_{2}}$ on $S\left(\nu_{3}\right)$ and so $2 \nu_{3}$ is the balayage of $\nu_{2}$ onto $S\left(\nu_{3}\right)$. Then the density of $\nu_{3}$ has a square root singularity at $\pm c_{3}$ and then it easily follows that

$$
\frac{d}{d x} U^{\nu_{3}}(x)=\int \frac{d \nu_{3}(s)}{s-x} \rightarrow+\infty \quad \text { as } x \rightarrow c_{3}+.
$$

This is not compatible with (3.18), since $U^{\nu_{2}}(x)-V_{3}(x)$ is differentiable at $x=c_{3}$. Therefore $c_{3}<x^{*}(\alpha)$ in case $x^{*}(\alpha)>0$.

Finally, if $c_{3}>0$ then the density of $\nu_{3}$ vanishes as a square root at $\pm c_{3}$ as a result of the convexity of $V_{3}(\sqrt{x})-U^{\nu_{2}}(\sqrt{x})$ again. The proposition is proved.

\subsection{Equilibrium problem for $\nu_{1}$}

Given $\nu_{2}$ and $\nu_{3}$ the equilibrium problem for $\nu_{1}$ is to minimize

$$
I(\nu)+\int\left(V(x)-\theta_{1}(x)-U^{\nu_{2}}(x)\right) d \nu(x)
$$

among all probability measures $\nu$ on $\mathbb{R}$. The minimizer exists and its support is contained in an interval $[-X, X]$ that is independent of $\nu_{2}$.

This can be proved as in [45], where weighted polynomials were used. Here we give a proof using potential theory.

LEMma 3.5. Let $\tilde{\nu}_{1}$ be the minimizer of the weighted energy

$$
I(\nu)+\int\left(V(x)-\theta_{1}(x)\right) d \nu(x)
$$

among probability measures on $\mathbb{R}$, and suppose that

$$
S\left(\tilde{\nu}_{1}\right) \subset\left[-X_{1}, X_{1}\right]
$$

for some $X_{1}>0$. Let $\nu_{2}$ be a measure on $i \mathbb{R}$, with finite potential $U^{\nu_{2}}$ and suppose that $\nu_{1}$ is the minimizer for (3.19). Then also

$$
S\left(\nu_{1}\right) \subset\left[-X_{1}, X_{1}\right] .
$$

PROOF. The variational conditions associated with the equilibrium problems for (3.20) and (3.19) are

$$
\left\{\begin{array}{l}
2 U^{\tilde{\nu}_{1}}(x)+V_{1}(x)=\tilde{\ell}_{1}, \quad x \in S\left(\tilde{\nu}_{1}\right), \\
2 U^{\tilde{\nu}_{1}}(x)+V_{1}(x) \geq \tilde{\ell}_{1}, \quad x \in \mathbb{R}
\end{array}\right.
$$


and

$$
\begin{cases}2 U^{\nu_{1}}(x)+V_{1}(x)-U^{\nu_{2}}(x)=\ell_{1}, & x \in S\left(\nu_{1}\right), \\ 2 U^{\nu_{1}}(x)+V_{1}(x)-U^{\nu_{2}}(x) \geq \ell_{1}, & x \in \mathbb{R},\end{cases}
$$

where $\tilde{\ell}_{1}$ and $\ell_{1}$ are certain constants. Combining the relatons (3.23) and (3.24), we find

$$
\begin{cases}2 U^{\tilde{\nu}_{1}}(x)-2 U^{\nu_{1}}(x)+U^{\nu_{2}}(x) \leq \tilde{\ell}_{1}-\ell_{1}, & x \in S\left(\tilde{\nu}_{1}\right), \\ 2 U^{\nu_{1}}(x)-2 U^{\tilde{\nu}_{1}}(x)-U^{\nu_{2}}(x) \leq \ell_{1}-\tilde{\ell}_{1}, & x \in S\left(\nu_{1}\right) .\end{cases}
$$

Since $x \mapsto-U^{\nu_{2}}(x)=\int \log |x-z| d \nu_{2}(z)$ is strictly increasing as $|x|$ increases, and since $S\left(\tilde{\nu}_{1}\right) \subset\left[-X_{1}, X_{1}\right]$, it follows from the first inequality in (3.25) that

$$
2 U^{\tilde{\nu}_{1}}(x) \leq 2 U^{\nu_{1}}(x)+\tilde{\ell}_{1}-\ell_{1}-U^{\nu_{2}}\left(X_{1}\right), \quad x \in S\left(\tilde{\nu}_{1}\right) .
$$

By the principle of domination, Theorem 3.1, the inequality holds everywhere

$$
2 U^{\tilde{\nu}_{1}}(x) \leq 2 U^{\nu_{1}}(x)+\tilde{\ell}_{1}-\ell_{1}-U^{\nu_{2}}\left(X_{1}\right), \quad x \in \mathbb{C} .
$$

Then for $x \in S\left(\nu_{1}\right)$ we find by combining this with the second inequality in (3.25)

$$
-U^{\nu_{2}}(x) \leq 2 U^{\tilde{\nu_{1}}}(x)-2 U^{\nu_{1}}(x)+\ell_{1}-\tilde{\ell}_{1} \leq-U^{\nu_{2}}\left(X_{1}\right)
$$

which implies that $|x| \leq X_{1}$, since $x \mapsto-U^{\nu_{2}}(x)$ is even and strictly increasing as $|x|$ increases.

\subsection{Equilibrium problem for $\nu_{2}$}

Given $\nu_{1}$ and $\nu_{3}$ on $\mathbb{R}$ with total masses $\int d \nu_{1}=1, \int d \nu_{3}=1 / 3$, the equilibrium problem for $\nu_{2}$ is to minimize

$$
I(\nu)-\int\left(U^{\nu_{1}}+U^{\nu_{3}}\right) d \nu
$$

among all measures on $i \mathbb{R}$ with $\nu \leq \sigma_{2}$ and $\int d \nu_{2}=2 / 3$.

Recall that by Lemma $1.2(\mathrm{~b})$ the density $d \sigma_{2}(i y) /|d z|$ increases as $y>0$ increases. Then we can use exactly the same arguments as in Lemma 4.5 of [45] to conclude that

$$
S\left(\nu_{2}\right)=S\left(\sigma_{2}\right)
$$

and

$$
S\left(\sigma_{2}-\nu_{2}\right)=\left(-i \infty,-i c_{2}\right] \cup\left[i c_{2}, i \infty\right)
$$

for some $c_{2} \geq 0$. The proof is based on iterated balayage introduced in [65. This proof also shows the following analogue of Lemma 3.3

LEMMA 3.6. Let $\nu_{1}$ and $\nu_{3}$ be measures on $\mathbb{R}$ with $\int d \nu_{1}=1$ and $\int d \nu_{3}=1 / 3$, and having finite logarithmic energy. Let $\nu_{2}$ be the minimizer of (3.26) among all measures $\nu$ on $i \mathbb{R}$ with total mass $2 / 3$ and $\nu \leq \sigma_{2}$.

Let $c \geq c_{2}$ and put

$$
B_{c}=(-i \infty,-i c] \cup[i c, i \infty) .
$$

Then we have

$$
\left.\left(\nu_{2}\right)\right|_{B_{c}} \geq\left.\frac{1}{2} \operatorname{Bal}\left(\nu_{1}+\nu_{3}, i \mathbb{R}\right)\right|_{B_{c}} .
$$


and

$$
\left.\left(\nu_{2}\right)\right|_{B_{c}} \leq \frac{1}{2} \operatorname{Bal}\left(\nu_{1}+\nu_{3}, B_{c}\right) .
$$

Proof. This follows from the iterated balayage. Alternatively, it could be proved from the variational conditions associated with the minimization problems, as we did for Lemma 3.3. We omit details.

\subsection{Uniqueness of the minimizer}

We write the energy functional (1.12) as

$$
\begin{aligned}
E\left(\nu_{1}, \nu_{2}, \nu_{3}\right)=\frac{2}{3} I\left(\nu_{1}\right)+\frac{1}{12} I\left(2 \nu_{1}-\right. & \left.3 \nu_{2}\right)+\frac{1}{4} I\left(\nu_{2}-2 \nu_{3}\right) \\
& +\int V_{1}(x) d \nu_{1}(x)+\int V_{3}(x) d \nu_{3}(x) .
\end{aligned}
$$

where $2 \nu_{1}-3 \nu_{2}$ and $\nu_{2}-2 \nu_{3}$ are signed measures with vanishing integral. From this the uniqueness follows as in Section 4.5 of [45. Note that there is a mistake in formula (4.21) of [45], since the coefficients of $I\left(\nu_{1}\right)$ and $I\left(2 \nu_{1}-3 \nu_{2}\right)$ are incorrect. However, the only important issue to establish uniqueness is that the coefficients are positive.

\subsection{Existence of the minimizer}

After these preparations we are able to show that the minimizer exists. The proof follows along the lines of Section 4.6 of 45 .

We fix $p \in(1,5 / 3)$. We are going to minimize the energy functional (3.32) among all measures $\nu_{1}, \nu_{2}, \nu_{3}$ as before, but with the additional restrictions that for certain given $X>0$ and $K>0$,

$$
\begin{gathered}
S\left(\nu_{1}\right) \subset[-X, X], \\
\frac{d \nu_{2}}{|d z|} \leq \frac{K}{|z|^{p}} \quad \text { for } z \in i \mathbb{R},|z| \geq X, \\
\frac{d \nu_{3}}{d x} \leq \frac{K}{x^{p}} \quad \text { for } x \in \mathbb{R},|x| \geq X .
\end{gathered}
$$

The constants $X$ and $K$ are at our disposal, and later we will choose them large enough.

For any choice of $X$ and $K$ there is a unique vector of measures that minimizes the energy functional subject to the usual constraints as well as the additional restrictions (3.33), (3.34), (3.35). Indeed, the additional restrictions yield that the measures are restricted to a tight sets of measures. The energy functional (3.32) is strictly convex, and so there is indeed a unique minimizer.

Our strategy of proof is now to show that for large enough $X$ and $K$ the additional restrictions are not effective.

Restriction (3.33). This is easy to do for (3.33). Indeed because of Lemma 3.5 it suffices to choose

$$
X>X_{1}
$$

where $X_{1}$ is as in the lemma. Then it is easy to see that (3.33) provides no extra restriction. 
Restriction (3.35) . The assumption $p \in(1,5 / 3)$ ensures that

$$
C_{p}=\frac{1}{\pi} \int_{0}^{\infty} \frac{s^{1-p}}{1+s^{2}} d s=\frac{1}{2 \sin (p \pi / 2)}<1 .
$$

Choose $\varepsilon_{p}>0$ such that

$$
\left(1+\varepsilon_{p}\right)^{2} C_{p}<1
$$

Pick $c \geq 0$ such that

$$
c \geq x^{*}(\alpha) \quad \text { and } \quad \sigma_{2}([-i c, i c]) \geq 2 / 3 .
$$

We take $X>X_{1}$ such that

$$
\frac{|x|}{\sqrt{x^{2}-c^{2}}} \leq 1+\varepsilon_{p}, \quad|x| \geq X
$$

Then $X>c$ and also

$$
\frac{\sqrt{c^{2}+x^{2}}}{|x|} \leq 1+\varepsilon_{p}, \quad|x| \geq X .
$$

Assume that $\nu_{2}$ is a measure on $i \mathbb{R}$, symmetric around the origin with $\int d \nu_{2}$ and satisfying the restriction (3.34) as well as $\nu_{2} \leq \sigma_{2}$. Let $\nu_{3}$ be the minimizer of (3.8) among measures $\nu$ on $\mathbb{R}$ with $\int d \nu=1 / 3$. Note that we do not impose the restriction (3.35).

Since $c \geq x^{*}(\alpha)$ we have the inequality (3.15) which due to the symmetry of $\nu_{2}$ may be written as

$$
\frac{d \nu_{3}(x)}{d x} \leq \frac{1}{\pi} \frac{|x|}{\sqrt{x^{2}-c^{2}}} \int_{0}^{i \infty} \frac{\sqrt{c^{2}+|z|^{2}}}{x^{2}+|z|^{2}} d \nu_{2}(z), \quad x \in \mathbb{R},|x|>c .
$$

Let $x \geq X>c$. We split the integral in (3.40) into an integral from 0 to $i X$ and from $i X$ to $i \infty$, and we estimate using (3.38)

$$
\begin{aligned}
\frac{1}{\pi} \frac{x}{\sqrt{x^{2}-c^{2}}} \int_{0}^{i X} \frac{\sqrt{c^{2}+|z|^{2}}}{x^{2}+|z|^{2}} d \nu_{2}(z) & \leq \frac{1+\varepsilon_{p}}{\pi}\left(\max _{z \in[0, i X]} \frac{\sqrt{c^{2}+|z|^{2}}}{x^{2}+|z|^{2}}\right) \nu_{2}([0, i X]) \\
& \leq \frac{1+\varepsilon_{p}}{3 \pi} \frac{\sqrt{c^{2}+X^{2}}}{x^{2}}
\end{aligned}
$$

and using (3.38) and (3.39)

$$
\begin{aligned}
\frac{1}{\pi} \frac{x}{\sqrt{x^{2}-c^{2}}} \int_{i X}^{i \infty} \frac{\sqrt{c^{2}+|z|^{2}}}{x^{2}+|z|^{2}} d \nu_{2}(z) & \leq \frac{\left(1+\varepsilon_{p}\right)^{2}}{\pi} \int_{i X}^{i \infty} \frac{|z|}{x^{2}+|z|^{2}} d \nu_{2}(z) \\
& \leq \frac{\left(1+\varepsilon_{p}\right)^{2}}{\pi} \int_{i X}^{i \infty} \frac{|z|}{x^{2}+|z|^{2}} \frac{K}{|z|^{p}}|d z|
\end{aligned}
$$

where the last inequality holds since $\nu_{2}$ satisfies (3.34). This leads to

$$
\begin{aligned}
\frac{1}{\pi} \frac{x}{\sqrt{x^{2}-c^{2}}} \int_{i X}^{i \infty} \frac{\sqrt{c^{2}+|z|^{2}}}{x^{2}+|z|^{2}} d \nu_{2}(z) & \leq \frac{\left(1+\varepsilon_{p}\right)^{2} K}{\pi} \int_{0}^{i \infty} \frac{|z|^{1-p}}{x^{2}+|z|^{2}}|d z| \\
& =\frac{\left(1+\varepsilon_{p}\right)^{2} K}{\pi|x|^{p}} \int_{0}^{\infty} \frac{s^{1-p}}{1+s^{2}} d s
\end{aligned}
$$

where we made the change of variables $z=i s, s \geq 0$. Thus by (3.36) we have

$$
\frac{1}{\pi} \frac{x}{\sqrt{x^{2}-c^{2}}} \int_{i X}^{i \infty} \frac{\sqrt{c^{2}+|z|^{2}}}{x^{2}+|z|^{2}} d \nu_{2}(z) \leq \frac{\left(1+\varepsilon_{p}\right)^{2} C_{p} K}{|x|^{p}}, \quad x \in \mathbb{R},|x| \geq X .
$$


In total we get

$$
\frac{d \nu_{3}(x)}{d x} \leq \frac{1+\varepsilon_{p}}{3 \pi} \frac{\sqrt{c^{2}+X^{2}}}{x^{2}}+\frac{\left(1+\varepsilon_{p}\right)^{2} C_{p} K}{|x|^{p}}, \quad x \in \mathbb{R},|x| \geq X .
$$

Since $\left(1+\varepsilon_{p}\right)^{2} C_{p}<1$ and $p<5 / 3<2$, it is possible to take now $K$ sufficiently large, say $K \geq K_{3}$, such that

$$
\frac{\left(1+\varepsilon_{p}\right)^{2}}{3 \pi} \frac{\sqrt{c^{2}+X^{2}}}{x^{2}}+\frac{\left(1+\varepsilon_{p}\right)^{2} C_{p} K}{|x|^{p}} \leq \frac{K}{|x|^{p}}, \quad x \in \mathbb{R},|x| \geq X .
$$

Then the additional restriction (3.35) is satisfied.

Restriction (3.34). A similar argument shows the following. Choose $\nu_{1}$ and $\nu_{3}$ on $\mathbb{R}$ with $\int d \nu_{1}=1, \int d \nu_{3}=1 / 3$ and satisfying the additional restrictions (3.33) and (3.35). Let $\nu_{2}$ be the minimizer for (3.26) for $\nu_{2}$ on $i \mathbb{R}$ with $\int d \nu_{2}=2 / 3$ and $\nu_{2} \leq \sigma_{2}$. However, we do not impose (3.34).

Then with the same choice for $X>X_{1}$ as above (so that (3.38) holds), we will find that for $K$ large enough, say $K \geq K_{2}$ we have that

$$
\frac{d \nu_{2}}{|d z|} \leq \frac{K}{|z|^{p}}, \quad z \in i \mathbb{R},|z| \geq X
$$

That is, the restriction (3.34) is satisfied.

This completes the proof of existence of the minimizer for the vector equilibrium problem.

\subsection{Proof of Theorem 1.1}

After all this work the proof of Theorem 1.1 is fairly short.

Proof. Existence and uniqueness of the minimizer is proved in Sections 3.5 and 3.6. We denote the minimizer by $\left(\mu_{1}, \mu_{2}, \mu_{3}\right)$.

The measure $\mu_{1}$ is the equilibrium measure in external field $V_{1}-U^{\mu_{2}}$, which is real analytic on $\mathbb{R} \backslash\{0\}$. If $0 \notin S\left(\mu_{1}\right)$, then this implies by a result of Deift, Kriecherbauer and McLaughlin 32 that $S\left(\mu_{1}\right)$ is a finite union of intervals with a density that has the form (1.25). If $0 \notin S\left(\sigma_{2}-\mu_{2}\right)$ then $V_{1}-U^{\mu_{2}}$ is real analytic on $\mathbb{R}$ (also at 0 ) by Lemma 1.2 (b), and again by 32 we find that $S\left(\mu_{1}\right)$ is a finite union of intervals with a density (1.25). The conditions (1.23) are the EulerLagrange conditions associated with the minimization in external field, and they are valid in all cases. This proves part (a).

The statements (1.27) about the supports of $\mu_{2}$ and $\sigma_{2}-\mu_{2}$ were already proved in Section 3.4, see (3.27) and (3.28). The Euler-Lagrange conditions (1.26) also follow from this. The fact that $\rho_{2}$ vanishes as a square root at $\pm i c_{2}$ in case $c_{2}>0$ follows as in the proof of Lemma 4.5 of $4 \mathbf{4 5}$. The other statements in part (b) are obvious.

Part (c) follows from Proposition 3.4, see also (3.18). This completes the proof of Theorem 1.1 


\section{CHAPTER 4}

\section{A Riemann Surface}

The rest of the paper is aimed at the proof of Theorem 1.4 We assume from now on that $(V, W, \tau)$ is regular, which means in particular that $S\left(\mu_{1}\right)$ and $S\left(\sigma_{2}-\mu_{2}\right)$ are disjoint. Then by part (a) of Theorem 1.1 we have that $S\left(\mu_{1}\right)$ consists of a finite union of disjoint intervals. We use this structure as well as that of the supports of the other measures to build a Riemann surface in this chapter.

We start by collecting consequences of the vector equilibrium problem in the form of properties of the $g$-functions. These will be used in the construction of a meromorphic function $\xi$ on the Riemann surface.

\subsection{The $g$-functions}

The Euler-Lagrange variational conditions (1.23), (1.26), (1.29), can be rewritten in terms of the $g$-functions

$$
g_{j}(z)=\int \log (z-s) d \mu_{j}(s), \quad j=1,2,3
$$

that are defined as follows.

Definition 4.1. For $j=1,2,3$ we define $g_{j}$ by the formula (4.1) with the following choice of branch for $\log (z-s)$ with $s \in S\left(\mu_{j}\right)$.

(a) For $j=1,3$, we define $\log (z-s)$ for $s \in \mathbb{R}$ with a branch cut along $(-\infty, s]$ on the real line.

(b) For $j=2$, we define $\log (z-s)$ for $s \in i \mathbb{R}$ with a branch cut along $(-\infty, 0] \cup[0, s]$, which is partly on the real line and partly on the imaginary axis.

In all cases the definition of $\log (z-s)$ is such that

$$
\log (z-s) \sim \log |z|+i \arg z, \quad-\pi<\arg z<\pi
$$

as $z \rightarrow \infty$.

As a result we have that $g_{1}$ is defined and analytic on $\mathbb{C} \backslash\left(-\infty, b_{N}\right], g_{2}$ on $\mathbb{C} \backslash\left(i \mathbb{R} \cup \mathbb{R}^{-}\right)$, and $g_{3}$ on $\mathbb{C} \backslash \mathbb{R}$.

In what follows we will frequently use the numbers $\alpha_{k}$ given in the following definition.

Definition 4.2. We define

$$
\alpha_{k}=\mu_{1}\left(\left[a_{k+1},+\infty\right)\right), \quad k=0, \ldots, N-1,
$$

and $\alpha_{N}=0$. and so

Recall that $\mu_{1}$ is supported on $\bigcup_{k=1}^{N}\left[a_{k}, b_{k}\right]$ with $a_{1}<b_{1}<a_{2}<\cdots<a_{N}<b_{N}$,

$$
\alpha_{1}=1>\alpha_{2}>\cdots>\alpha_{N-1}>\alpha_{N}=0 \text {. }
$$


The above definitions are such that the following hold.

LEMma 4.3. (a) We have

$$
g_{1,+}(x)-g_{1,-}(x)=2 \pi i \mu_{1}([x, \infty)) \quad \text { for } x \in \mathbb{R} .
$$

In particular

$$
g_{1,+}-g_{1,-}=2 \pi i \alpha_{k}, \quad \text { on }\left(b_{k}, a_{k+1}\right)
$$

with $\alpha_{k}$ as in (4.2). Here we have put $b_{0}=-\infty$ and $a_{N+1}=+\infty$.

(b) We have

$$
g_{2, \pm}(x)=\int \log |x-s| d \mu_{2}(s) \pm \frac{2}{3} \pi i, \quad x \in \mathbb{R}^{-},
$$

so that

$$
g_{2,+}-g_{2,-}=\frac{4}{3} \pi i, \quad \text { on } \mathbb{R}^{-} .
$$

(c) If $c_{2}>0$ then

$$
\begin{cases}g_{2,+}(z)-g_{2,-}(z)=\frac{2}{3} \pi i-2 \pi i \sigma_{2}([0, z]), & z \in\left(0, i c_{2}\right), \\ g_{2,+}(z)-g_{2,-}(z)=-\frac{2}{3} \pi i+2 \pi i \sigma_{2}([z, 0]), & z \in\left(-i c_{2}, 0\right) .\end{cases}
$$

(d) We have

$$
g_{3,+}(x)-g_{3,-}(x)=2 \pi i \mu_{3}([x, \infty)), \quad x \in \mathbb{R} .
$$

(e) If $c_{3}>0$, then

$$
g_{3, \pm}(x)=\int \log |x-s| d \mu_{3}(s) \pm \frac{1}{6} \pi i, \quad x \in\left(-c_{3}, c_{3}\right),
$$

so that

$$
g_{3,+}-g_{3,-}=\frac{1}{3} \pi i, \quad \text { on }\left(-c_{3}, c_{3}\right) .
$$

Proof. Parts (a) and (d) are immediate from (4.1).

Part (b) follows from the definition of $g_{2}$, the symmetry of the measure $\mu_{2}$ on $i \mathbb{R}$, and the fact that $\int d \mu_{2}=2 / 3$.

For part (c), we note that for $z \in i \mathbb{R}^{+}$,

$$
g_{2, \pm}(z)=-U^{\mu_{2}}(z)+\frac{1}{3} \pi i \pm \pi i \mu_{2}([z, i \infty)),
$$

such that

$$
g_{2,+}(z)-g_{2,-}(z)=2 \pi i \mu_{2}([z, i \infty))=\frac{2}{3} \pi i-2 \pi i \mu_{2}([0, z])
$$

since $\mu_{2}$ has total mass $1 / 3$ on $i \mathbb{R}^{+}$. If $z \in\left[0, i c_{2}\right]$, then $\mu_{2}([0, z])=\sigma_{2}([0, z])$ and the first equation in (4.6) follows. The second equation follows in a similar way.

Part (e) follows from the definition of $g_{3}$, together with the fact that $\mu_{3}$ is symmetric on $\mathbb{R}$ with total mass $1 / 3$, so that $\mu_{3}\left(\left[c_{3}, \infty\right)\right)=1 / 6$.

We have the following jump properties. 
Lemma 4.4. (a) We have

$$
\left\{\begin{aligned}
g_{1,+}+g_{1,-}-g_{2} & =V_{1}-\ell_{1}, & & \text { on } S\left(\mu_{1}\right) \cap \mathbb{R}^{+}, \\
g_{1,+}+g_{1,-}-g_{2, \pm} & =V_{1}-\ell_{1} \mp \frac{2}{3} \pi i, & & \text { on } S\left(\mu_{1}\right) \cap \mathbb{R}^{-}, \\
\operatorname{Re}\left(g_{1,+}+g_{1,-}-g_{2}\right) & \leq V_{1}-\ell_{1}, & & \text { on } \mathbb{R} \backslash S\left(\mu_{1}\right) .
\end{aligned}\right.
$$

(b) On $i \mathbb{R}$ we have

$$
\left\{\begin{array}{clrl}
g_{2,+}+g_{2,-} & =g_{1}+g_{3}, & & \text { on } S\left(\sigma_{2}-\mu_{2}\right), \\
\operatorname{Re}\left(g_{2,+}+g_{2,-}\right)>\operatorname{Re}\left(g_{1}+g_{3}\right), & & \text { on } i \mathbb{R} \backslash S\left(\sigma_{2}-\mu_{2}\right) .
\end{array}\right.
$$

(c) We have on $\mathbb{R}$,

$$
\left\{\begin{aligned}
g_{3,+}+g_{3,-}-g_{2} & =V_{3}, & & \text { on } S\left(\mu_{3}\right) \cap \mathbb{R}^{+}, \\
g_{3,+}+g_{3,-}-g_{2, \pm} & =V_{3} \mp \frac{2}{3} \pi i, & & \text { on } S\left(\mu_{3}\right) \cap \mathbb{R}^{-}, \\
\operatorname{Re}\left(g_{3,+}+g_{3,-}-g_{2}\right) & <V_{3}, & & \text { on } \mathbb{R} \backslash S\left(\mu_{3}\right) .
\end{aligned}\right.
$$

Proof. Part (a) follows from (1.23). Indeed, we have

$$
\begin{aligned}
& 2 U^{\mu_{1}}(x)=-g_{1,+}(x)-g_{1,-}(x), \quad \text { for } x \in \mathbb{R}, \\
& U^{\mu_{2}}(x)= \begin{cases}-g_{2}(x), & \text { for } x \in \mathbb{R}^{+}, \\
-g_{2, \pm}(x) \pm \frac{2}{3} \pi i, & \text { for } x \in \mathbb{R}^{-},\end{cases}
\end{aligned}
$$

so that (1.23) indeed leads to (4.10).

For part (b) we note that

$$
2 U^{\mu_{2}}(z)=-g_{2,+}(z)-g_{2,-}(z) \pm \frac{2}{3} \pi i, \quad z \in i \mathbb{R}^{ \pm} .
$$

Also because of symmetry of $g_{1}$ and $g_{3}$ around 0 ,

$$
\left\{\begin{array}{l}
U^{\mu_{1}}(z)=-g_{1}(z) \pm \frac{1}{2} \pi i, \\
U^{\mu_{3}}(z)=-g_{3}(z) \pm \frac{1}{6} \pi i,
\end{array} \text { for } z \in i \mathbb{R}^{ \pm} .\right.
$$

Using this in (1.26) we obtain (4.11).

Part (c) follows from (1.29) in the same way that we obtained part (a) from (1.23).

In what follows we also use the derivatives of the $g$-function, which we denote by $F_{1}, F_{2}, F_{3}$.

Definition 4.5. We define

$$
F_{j}(z)=g_{j}^{\prime}(z)=\int \frac{d \mu_{j}(s)}{z-s}, \quad j=1,2,3,
$$

which is defined and analytic for $z \in \mathbb{C} \backslash S\left(\mu_{j}\right)$. 
The jump of $F_{j}$ gives us the density of $\mu_{j}$, since we have

$$
\begin{array}{lll}
\frac{d \mu_{1}}{d x}=-\frac{1}{2 \pi i}\left(F_{1,+}(x)-F_{1,-}(x)\right), & & x \in \mathbb{R}, \\
\frac{d \mu_{2}}{d z}=-\frac{1}{2 \pi i}\left(F_{2,+}(z)-F_{2,-}(z)\right), & & z \in i \mathbb{R}, \\
\frac{d \mu_{3}}{d x}=-\frac{1}{2 \pi i}\left(F_{3,+}(x)-F_{3,-}(x)\right), & & x \in \mathbb{R} .
\end{array}
$$

\subsection{Riemann surface $\mathcal{R}$ and $\xi$-functions}

We construct a four sheeted Riemann surface $\mathcal{R}$ in the following way. Four sheets $\mathcal{R}_{j}$ defined as

$$
\left\{\begin{array}{l}
\mathcal{R}_{1}=\mathbb{C} \backslash S\left(\mu_{1}\right), \\
\mathcal{R}_{2}=\mathbb{C} \backslash\left(S\left(\mu_{1}\right) \cup S\left(\sigma_{2}-\mu_{2}\right)\right), \\
\mathcal{R}_{3}=\mathbb{C} \backslash\left(S\left(\sigma_{2}-\mu_{2}\right) \cup S\left(\mu_{3}\right)\right), \\
\mathcal{R}_{4}=\mathbb{C} \backslash S\left(\mu_{3}\right),
\end{array}\right.
$$

are connected as follows: $\mathcal{R}_{1}$ is connected to $\mathcal{R}_{2}$ via $S\left(\mu_{1}\right), \mathcal{R}_{2}$ is connected to $\mathcal{R}_{3}$ via $S\left(\sigma_{2}-\mu_{2}\right)$ and $\mathcal{R}_{3}$ is connected to $\mathcal{R}_{4}$ via $S\left(\mu_{3}\right)$. Every connection is in the usual crosswise manner. We compactify the Riemann surface by adding a point at infinity to the first sheet $\mathcal{R}_{1}$, and a second point at infinity which is common to the other three sheets.

The genus of the Riemann surface is (recall that $S\left(\mu_{1}\right)$ consists of $N$ intervals and recall the classification of the cases in Section 1.5)

$$
g(\mathcal{R})= \begin{cases}N-1, & \text { in Cases I, II, and III, } \\ N, & \text { in Cases IV and V. }\end{cases}
$$

Using the functions $F_{j}$ defined in Definition 4.5 we define the $\xi$ functions.

Definition 4.6. We define functions $\xi_{j}, j=1,2,3,4$ by

$$
\begin{cases}\xi_{1}=V^{\prime}-F_{1}, & \text { on } \mathcal{R}_{1}, \\ \xi_{2}=F_{1}-F_{2}+\theta_{1}^{\prime}, & \text { on } \mathcal{R}_{2} \backslash i \mathbb{R}, \\ \xi_{3}=F_{2}-F_{3}+\theta_{2}^{\prime}, & \text { on } \mathcal{R}_{3} \backslash(\mathbb{R} \cup i \mathbb{R}), \\ \xi_{4}=F_{3}+\theta_{3}^{\prime}, & \text { on } \mathcal{R}_{4} \backslash \mathbb{R} .\end{cases}
$$

We first prove that $\xi_{2}, \xi_{3}$, and $\xi_{4}$ are analytic on their full respective sheets.

Lemma 4.7. (a) We have $\xi_{2,+}=\xi_{2,-}$ on $\left(-i c_{2}, i c_{2}\right)$, and so $\xi_{2}$ has an analytic extension to $\mathcal{R}_{2}$.

(b) We have $\xi_{3,+}=\xi_{3,-}$ on $\left(-i c_{2}, i c_{2}\right)$ and on $\left(-c_{3}, c_{3}\right)$, and so $\xi_{3}$ has an analytic extension to $\mathcal{R}_{3}$.

(c) We have $\xi_{4,+}=\xi_{4,-}$ on $\left(-c_{3}, c_{3}\right)$, and so $\xi_{4}$ has an analytic extension to $\mathcal{R}_{4}$.

Proof. On $\left(-i c_{2}, i c_{2}\right)$ we have by (4.6) and (4.13) that

$$
\begin{aligned}
F_{2,+}(z)-F_{2,-}(z) & =\frac{d}{d z}\left(g_{2,+}(z)-g_{2,-}(z)\right) \\
& =-2 \pi i \frac{d \sigma_{2}}{d z}
\end{aligned}
$$




$$
=\tau\left(s_{1,+}(z)-s_{1,-}(z)\right), \quad z \in\left(-i c_{2}, i c_{2}\right),
$$

see (2.7). Since $\tau s_{1}=\theta_{1}^{\prime}$, we get

$$
F_{2,+}-\theta_{1,+}=F_{2,-}-\theta_{1,-}^{\prime}, \quad \text { on }\left(-i c_{2}, i c_{2}\right),
$$

and also

$$
\xi_{2,+}=\xi_{2,-}, \quad \text { on }\left(-i c_{2}, i c_{2}\right)
$$

since $F_{1}$ is analytic on the imaginary axis. This proves part (a).

We also have

$$
s_{1,+}(z)-s_{1,-}(z)=s_{2,-}(z)-s_{2,+}(z), \quad z \in i \mathbb{R} .
$$

Then above argument also shows that

$$
\xi_{3,+}=\xi_{3,-}, \quad \text { on }\left(-i c_{2}, i c_{2}\right)
$$

since $F_{1}$ and $F_{3}$ are analytic on the imaginary axis.

If $c_{3}>0$ (which can only happen if $\alpha<0$ ), then $F_{3}$ is analytic across $\left(-c_{3}, c_{3}\right)$. Both $\xi_{2}$ and $\xi_{3}$ are analytic across $\left(-x^{*}(\alpha), x^{*}(\alpha)\right)$, and so a fortiori across $\left(-c_{3}, c_{3}\right)$, since $c_{3}<x^{*}(\alpha)$. This proves part (c) and the remaining statement of part (b).

We continue to denote the analytic extension by $\xi_{j}, j=2,3,4$.

Proposition 4.8. The function

$$
\xi: \bigcup_{j=1}^{4} \mathcal{R}_{j} \rightarrow \mathbb{C}
$$

given by $\xi(z)=\xi_{j}(z)$ for $z \in \mathcal{R}_{j}$ extends to a meromorphic function (also denoted by $\xi$ ) on $\mathcal{R}$. The meromorphic function has a pole of order $\operatorname{deg} V-1$ at infinity on the first sheet, and a simple pole at the other point at infinity.

Proof. From (4.10) and (4.13) it follows that

$$
F_{1,+}(x)+F_{1,-}(x)-F_{2}(x)=V_{1}^{\prime}(x), \quad x \in S\left(\mu_{1}\right),
$$

since $F_{2}$ is analytic on $\mathbb{R} \backslash\{0\}$, so that $F_{2, \pm}=F_{2}$. Since $V_{1}=V-\theta_{1}$, we obtain by the definition (4.17) that

$$
\xi_{1, \pm}(x)=\xi_{2, \mp}(x), \quad x \in S\left(\mu_{1}\right) .
$$

From the first equation in (2.10) and the fact that $c_{2} \geq y^{*}(\alpha)$, we obtain that

$$
\theta_{1, \pm}=\theta_{2, \mp}, \quad \text { on } S\left(\sigma_{2}-\mu_{2}\right)=\left(-i \infty,-i c_{2}\right] \cup\left[i c_{2}, \infty\right) .
$$

Then we obtain from (4.11) that

$$
F_{2,+}(z)+F_{2,-}(z)=F_{1}(z)+F_{3}(z), \quad z \in S\left(\sigma_{2}-\mu_{2}\right),
$$

and it follows from (4.17) that

$$
\xi_{2, \pm}(z)=\xi_{3, \mp}(z), \quad z \in S\left(\sigma_{2}-\mu_{2}\right) .
$$

From (4.12) we similarly find

$$
F_{3,+}(x)+F_{3,-}(x)-F_{2}(x)=V_{3}^{\prime}(x), \quad x \in S\left(\mu_{3}\right) .
$$

We next claim that

$$
V_{3}(x)=\theta_{2, \pm}(x)-\theta_{3, \mp}(x), \quad x \in \mathbb{R} .
$$

Indeed, for $x \in\left(-x^{*}(\alpha), x^{*}(\alpha)\right)$ we have by (2.15) that $V_{3}(x)=\theta_{2}(x)-\theta_{3}(x)$ and (4.23) holds, while for $x \in \mathbb{R}$ with $|x| \geq x^{*}$, we have by (2.10) and (2.15) that 
both sides of (4.23) are equal to zero. Using (4.23) in (4.22) we obtain from the definition (4.17) that

$$
\xi_{3, \pm}(x)=\xi_{4, \mp}(x), \quad x \in S\left(\mu_{3}\right) .
$$

The analyticity of $\xi$ across the three cuts $S\left(\mu_{1}\right), S\left(\sigma_{2}-\mu_{2}\right)$ and $S\left(\mu_{3}\right)$ is now established by (4.19), (4.21), and (4.24).

As $z \rightarrow \infty$, we have by (4.17) and (4.13) that

$$
\xi_{1}(z)=V^{\prime}(z)+\mathcal{O}\left(z^{-1}\right)
$$

which implies that $\xi$ has a pole of order $\operatorname{deg} V-1$ at the point at infinity on the first sheet.

From (4.17), (4.13) and Lemma 2.4 it also follows that for $j=2,3,4$,

$$
\xi_{j}(z)=\omega^{k} \tau^{4 / 3} z^{1 / 3}+\mathcal{O}\left(z^{-1 / 3}\right), \quad \text { as } z \rightarrow \infty,
$$

where the value of $k$ depends on $j$ and on the quadrant in which $z \rightarrow \infty$. Since the other point at infinity (which is common to the second, third and fourth sheets) is a double branch point, we have that $z^{-1 / 3}$ is a local coordinate, so that $\xi$ indeed has a simple pole.

REMARK 4.9. It follows from Proposition 4.8 that $\xi_{j}, j=1,2,3,4$ are solutions of a quartic equation

$$
\xi^{4}+p_{3}(z) \xi^{3}+p_{2}(z) \xi^{2}+p_{1}(z) \xi+p_{0}(z)=0
$$

with coefficients that are polynomial in $z$. This algebraic equation is known as the spectral curve 13 . The degrees of the polynomial coefficients are determined by the degree of $V$.

Using this fact, we indicate how to remove the condition that $S\left(\mu_{1}\right)$ and $S\left(\sigma_{2}-\right.$ $\left.\mu_{2}\right)$ are disjoint in part (a) of Theorem 1.1. This condition was included in order to be able to conclude that $S\left(\mu_{1}\right)$ is a finite union of intervals. In case the condition does not hold, we can now argue as follows.

Given $\varepsilon>0$ we modify the vector equilibrium problem by requiring that $S\left(\mu_{1}\right) \subset(-\infty,-\varepsilon] \cup[\varepsilon, \infty)$. Then the proof of existence and uniqueness of the minimizer follows in the same way as in Section 3 , Let us denote the minimizer by $\left(\mu_{1}^{\varepsilon}, \mu_{2}^{\varepsilon}, \mu_{3}^{\varepsilon}\right)$. Because the external field $V_{1}-U^{\mu_{2}^{\varepsilon}}$ is real analytic on $\mathbb{R} \backslash(-\varepsilon, \varepsilon)$ we have that the support of $\mu_{1}^{\varepsilon}$ is a finite union of intervals. The further structure of the minimizers is the same, which means that we can construct a Riemann surface with a globally meromorphic function on it, in the same way as we did in this chapter. The only difference is that the meromorphic function may have a pole in $\pm \varepsilon$. The algebraic equation (4.25) has coefficients that are rational in $z$, with $\pm \varepsilon$ as the only (simple) poles, and so we may write the spectral curve as

$$
\left(z^{2}-\varepsilon^{2}\right) \xi^{4}+q_{3}(z) \xi^{3}+q_{2}(z) \xi^{2}+q_{1}(z) \xi+q_{0}(z)=0
$$

with polynomial coefficients $q_{j}(z)$, whose degrees is determined by the degree of $V$. In particular, the degrees do not depend on $\varepsilon$.

As $\varepsilon \rightarrow 0$ we have that $\mu_{j}^{\varepsilon}$ converges weakly to $\mu_{j}$ for $j=1,2,3$, and it is not difficult to show that the spectral curve (4.26) has a limit as well. Then $V_{1}^{\prime}(z)-\int \frac{d \mu_{1}(s)}{z-s}$ is the solution of an algebraic equation and therefore the support of $\mu_{1}$ is a finite union of intervals. 


\subsection{Properties of the $\xi$ functions}

From (4.14) and the definition (4.17) we find that

$$
\begin{aligned}
\frac{d \mu_{1}}{d x} & =\frac{1}{2 \pi i}\left(\xi_{1,+}(x)-\xi_{1,-}(x)\right), \quad x \in \mathbb{R}, \\
\frac{d \mu_{2}}{d z} & =\frac{1}{2 \pi i}\left(\xi_{2,+}(z)-\xi_{2,-}(z)\right)-\frac{1}{2 \pi i}\left(\theta_{1,+}^{\prime}(z)-\theta_{1,-}^{\prime}(z)\right) \\
& =-\frac{1}{2 \pi i}\left(\xi_{3,+}(z)-\xi_{3,-}(z)\right)+\frac{1}{2 \pi i}\left(\theta_{2,+}^{\prime}(z)-\theta_{2,-}^{\prime}(z)\right), \quad z \in i \mathbb{R}, \\
\frac{d \mu_{3}}{d x} & =\frac{1}{2 \pi i}\left(\xi_{3,+}(x)-\xi_{3,-}(x)\right)-\frac{1}{2 \pi i}\left(\theta_{2,+}^{\prime}(x)-\theta_{2,-}^{\prime}(x)\right) \\
& =-\frac{1}{2 \pi i}\left(\xi_{4,+}(x)-\xi_{4,-}(x)\right)+\frac{1}{2 \pi i}\left(\theta_{3,+}^{\prime}(x)-\theta_{3,-}^{\prime}(x)\right), \quad x \in \mathbb{R} .
\end{aligned}
$$

Since $\xi$ is an odd function with a simple pole at infinity on sheets 2,3 and 4 , there is an expansion in the local coordinate $z^{-1 / 3}$ :

$$
\xi(z)=c_{-1} z^{1 / 3}+c_{1} z^{-1 / 3}+c_{3} z^{-1}+c_{5} z^{-5 / 3}+\cdots
$$

with certain real constants $c_{j}$. Keeping track of the principal branches, we obtain the following for the asymptotic behavior of $\xi_{2}, \xi_{3}$ and $\xi_{4}$ as $z \rightarrow \infty$. Note that the structure of the formulas in terms of the factors $\omega$ and $\omega^{2}$ is the same as in Lemma 2.3 and Lemma 2.4

Lemma 4.10. We have as $z \rightarrow \infty$

$$
\begin{aligned}
& \xi_{2}(z)= \begin{cases}c_{-1} z^{1 / 3}+c_{1} z^{-1 / 3}+c_{3} z^{-1}+c_{5} z^{-5 / 3}+\cdots, & \text { in } I \cup I V, \\
c_{-1} \omega z^{1 / 3}+c_{1} \omega^{2} z^{-1 / 3}+c_{3} z^{-1}+c_{5} \omega z^{-5 / 3}+\cdots, & \text { in } I I, \\
c_{-1} \omega^{2} z^{1 / 3}+c_{1} \omega z^{-1 / 3}+c_{3} z^{-1}+c_{5} \omega^{2} z^{-5 / 3}+\cdots, & \text { in } I I I,\end{cases} \\
& \xi_{3}(z)= \begin{cases}c_{-1} \omega z^{1 / 3}+c_{1} \omega^{2} z^{-1 / 3}+c_{3} z^{-1}+c_{5} \omega z^{-5 / 3}+\cdots, & \text { in } I, \\
c_{-1} z^{1 / 3}+c_{1} z^{-1 / 3}+c_{3} z^{-1}+c_{5} z^{-5 / 3}+\cdots, & \text { in } I I \cup I I I, \\
c_{-1} \omega^{2} z^{1 / 3}+c_{1} \omega z^{-1 / 3}+c_{3} z^{-1}+c_{5} \omega^{2} z^{-5 / 3}+\cdots, & \text { in } I V,\end{cases} \\
& \xi_{4}(z)= \begin{cases}c_{-1} \omega^{2} z^{1 / 3}+c_{1} \omega z^{-1 / 3}+c_{3} z^{-1}+c_{5} \omega^{2} z^{-5 / 3}+\cdots, & \text { in } I \cup I I, \\
c_{-1} \omega z^{1 / 3}+c_{1} \omega^{2} z^{-1 / 3}+c_{3} z^{-1}+c_{5} \omega z^{-5 / 3}+\cdots, & \text { in } I I I \cup I V .\end{cases}
\end{aligned}
$$

with constants

$$
c_{-1}=\tau^{4 / 3}, \quad c_{1}=-\frac{\alpha}{3} \tau^{2 / 3}, \quad c_{3}=1 / 3 .
$$

Proof. The form of the asymptotics follows from the definition (4.17) snd Lemmas 2.3 and 2.4. Since $\mu_{1}$ is a probability measure with compact support, and symmetric with respect to the origin, we have

$$
F_{1}(z)=\frac{1}{z}+\mathcal{O}\left(z^{-3}\right)
$$

From

$$
F_{2}(z)=\theta_{1}^{\prime}(z)-\xi_{2}(z)+F_{1}(z)=\tau s_{1}(z)-\xi_{2}(z)+F_{1}(z)
$$

we see that $F_{2}$ has a series representation around $z=\infty$ in powers of $z^{1 / 3}$. It should start with $F_{2}(z)=\frac{2}{3} z^{-1}+\cdots$ since $\mu_{2}$ has total mass $2 / 3$, see (4.13). Then the coefficients for $z^{1 / 3}$ and $z^{-1 / 3}$ should vanish which by Lemma 2.3, and (4.30) 
leads to the expressions in (4.28) for $c_{-1}$ and $c_{1}$. The coefficient of $z^{-1}$ should be $2 / 3$, which by (4.29) and (4.30) leads to $c_{3}=1 / 3$.

Combining (4.29) and (4.30) with Lemmas 2.3 and 4.10 we also find that there is a real constant

$$
C=c_{5}-\frac{\alpha^{3}}{81} \tau^{-2 / 3}
$$

such that

$$
F_{2}(z)= \begin{cases}\frac{2}{3} z^{-1}-C z^{-5 / 3}+\mathcal{O}\left(z^{-2}\right), & z \in I \cup I V, \\ \frac{2}{3} z^{-1}-C \omega z^{-5 / 3}+\mathcal{O}\left(z^{-2}\right), & z \in I I, \\ \frac{2}{3} z^{-1}-C \omega^{2} z^{-5 / 3}+\mathcal{O}\left(z^{-2}\right), & z \in I I I\end{cases}
$$

as $z \rightarrow \infty$. Then by (4.17), and Lemmas 2.4 and 4.10, we also get

$$
F_{3}(z)= \begin{cases}\frac{1}{3} z^{-1}+C \omega^{2} z^{-5 / 3}+\mathcal{O}\left(z^{-2}\right), & z \in I \cup I I, \\ \frac{1}{3} z^{-1}+C \omega z^{-5 / 3}+\mathcal{O}\left(z^{-2}\right), & z \in I I I \cup I V,\end{cases}
$$

as $z \rightarrow \infty$, with the same constant $C$. Using this and Lemma 2.4 in (4.27) we find that

$$
\begin{array}{ll}
\frac{d \mu_{2}}{|d z|}=\frac{\sqrt{3}}{2 \pi} C|z|^{-5 / 3}+\mathcal{O}\left(z^{-2}\right), & \text { as }|z| \rightarrow \infty, z \in i \mathbb{R} \\
\frac{d \mu_{3}}{d x}=\frac{\sqrt{3}}{2 \pi} C x^{-5 / 3}+\mathcal{O}\left(x^{-2}\right), & \text { as } x \rightarrow \infty, x \in \mathbb{R} .
\end{array}
$$

which gives the precise rate of decay of the densities of $\mu_{2}$ and $\mu_{3}$ along the imaginary and real axis, respectively. It also follows from (4.34) that the constant is positive, $C>0$.

Furthermore, after integration, we find from (4.29), (4.32), and (4.33)

$$
\begin{aligned}
& g_{1}(z)=\log z+\mathcal{O}\left(z^{-1}\right) \\
& g_{2}(z)= \begin{cases}\frac{2}{3} \log z+\frac{3}{2} C z^{-2 / 3}+\mathcal{O}\left(z^{-1}\right), & z \in I \cup I V, \\
\frac{2}{3} \log z+\frac{3}{2} C \omega z^{-2 / 3}+\mathcal{O}\left(z^{-1}\right), & z \in I I, \\
\frac{2}{3} \log z+\frac{3}{2} C \omega^{2} z^{-2 / 3}+\mathcal{O}\left(z^{-1}\right), & z \in I I I,\end{cases} \\
& g_{3}(z)= \begin{cases}\frac{1}{3} \log z-\frac{3}{2} C \omega^{2} z^{-2 / 3}+\mathcal{O}\left(z^{-1}\right), & z \in I \cup I I, \\
\frac{1}{3} \log z-\frac{3}{2} C \omega z^{-2 / 3}+\mathcal{O}\left(z^{-1}\right), & z \in I I I \cup I V\end{cases}
\end{aligned}
$$

as $z \rightarrow \infty$. It may be verified from (4.1) that the constant of integration indeed vanishes.

\subsection{The $\lambda$ functions}

The $\lambda$ functions are primitive functions of the $\xi$ functions (that is, Abelian integrals). They are defined as follows.

Definition 4.11. We define

$$
\left\{\begin{array}{l}
\lambda_{1}=V-g_{1}-\ell_{1}, \\
\lambda_{2}=g_{1}-g_{2}+\theta_{1}, \\
\lambda_{3}=g_{2}-g_{3}+\theta_{2}, \\
\lambda_{4}=g_{3}+\theta_{3} .
\end{array}\right.
$$


It is convenient to consider each $\lambda_{j}$ function as defined on $\mathcal{R}_{j}$ with an extra cut to ensure single-valuedness. Thus, $\lambda_{1}$ is defined and analytic on $\mathbb{C} \backslash\left(-\infty, b_{N}\right]$, $\lambda_{2}$ on $\mathbb{C} \backslash\left(\left(-\infty, b_{N}\right] \cup i \mathbb{R}\right), \lambda_{3}$ on $\mathbb{C} \backslash(\mathbb{R} \cup i \mathbb{R})$, and $\lambda_{4}$ on $\mathbb{C} \backslash \mathbb{R}$.

The $\lambda$-functions satisfy the following jump conditions that follow by combining the jumps for the $g$-functions given in Lemmas 4.3 and 4.4 with the properties (2.10) of the functions $\theta_{j}$.

LEMma 4.12. (a) We have

$$
\begin{cases}\lambda_{1, \pm}-\lambda_{2, \mp}=0, & \text { on } S\left(\mu_{1}\right) \cap \mathbb{R}^{+}, \\ \lambda_{1, \pm}-\lambda_{2, \mp}=\mp \frac{2}{3} \pi i, & \text { on } S\left(\mu_{1}\right) \cap \mathbb{R}^{-} .\end{cases}
$$

and for $k=0, \ldots, N$,

$$
\begin{gathered}
\left\{\begin{array}{l}
\lambda_{1,+}-\lambda_{1,-}=-2 \pi i \alpha_{k}, \\
\lambda_{2,+}-\lambda_{2,-}=2 \pi i \alpha_{k},
\end{array} \quad \text { on }\left(b_{k}, a_{k+1}\right) \cap \mathbb{R}^{+},\right. \\
\left\{\begin{array}{l}
\lambda_{1,+}-\lambda_{1,-}=-2 \pi i \alpha_{k}, \\
\lambda_{2,+}-\lambda_{2,-}=2 \pi i \alpha_{k}-\frac{4}{3} \pi i,
\end{array}\right.
\end{gathered}
$$

where $b_{0}=-\infty, a_{N+1}=+\infty$, and $\alpha_{k}$ is given by (4.2) for $k=0, \ldots, N$.

(b) On the imaginary axis we have

$$
\lambda_{2, \pm}-\lambda_{3, \mp}=0, \quad \text { on } S\left(\sigma_{2}-\mu_{2}\right),
$$

and

$$
\left\{\begin{array}{l}
\lambda_{2,+}-\lambda_{2,-}=\mp \frac{2}{3} \pi i, \\
\lambda_{3,+}-\lambda_{3,-}= \pm \frac{2}{3} \pi i,
\end{array} \quad \text { on }\left(-i c_{2}, i c_{2}\right) \cap i \mathbb{R}^{ \pm} .\right.
$$

(c) Finally,

$$
\begin{cases}\lambda_{3, \pm}-\lambda_{4, \mp}=0, & \text { on }\left(c_{3}, \infty\right) \\ \lambda_{3, \pm}-\lambda_{4, \mp}=\mp \frac{2}{3} \pi i, & \text { on }\left(-\infty,-c_{3}\right)\end{cases}
$$

and

$$
\left\{\begin{array}{l}
\lambda_{3,+}-\lambda_{3,-}=-\frac{1}{3} \pi i, \\
\lambda_{4,+}-\lambda_{4,-}=\frac{1}{3} \pi i,
\end{array} \text { on }\left(-c_{3}, c_{3}\right) .\right.
$$

Proof. The equations follow by combining the jumps for the $g$-functions given in Lemmas 4.3 and 4.4 with the properties (2.10) of the functions $\theta_{j}$. Only (4.40) requires more explanation.

From (4.6) and (4.35) we get for $z \in\left(0, i c_{2}\right)$,

$$
\lambda_{2,+}(z)-\lambda_{2,-}(z)=-\frac{2}{3} \pi i+2 \pi i \sigma_{2}([0, z])+\left(\theta_{1,+}(z)-\theta_{1,-}(z)\right)
$$

where we used the fact that $g_{1}$ and $g_{3}$ are analytic on the imaginary axis. Since $\sigma_{2}$ has density (2.7) and $\theta_{1}^{\prime}=\tau s_{1}$, we have

$$
\sigma_{2}([0, z])=\int_{0}^{z} \frac{d \sigma_{2}}{d z}\left(z^{\prime}\right) d z^{\prime}=\frac{1}{2 \pi i} \int_{0}^{z}\left(\theta_{1,-}^{\prime}\left(z^{\prime}\right)-\theta_{1,+}^{\prime}\left(z^{\prime}\right)\right) d z^{\prime}
$$




$$
=\frac{1}{2 \pi i}\left(\theta_{1,-}(z)-\theta_{1,+}(z)-\theta_{1,-}(0)+\theta_{1,+}(0)\right)
$$

and the first equality in (4.40) follows for $z \in\left(0, i c_{2}\right)$ since $\theta_{1,-}(0)=\theta_{1,+}(0)$. The other equalities contained in (4.40) follow in the same way.

The $\lambda$ functions also satisfy a number of inequalities.

LEMma 4.13. We have the following inequalities

$$
\begin{array}{ll}
\operatorname{Re}\left(\lambda_{2,+}-\lambda_{1,-}\right)<0 & \text { on } \mathbb{R} \backslash S\left(\mu_{1}\right), \\
\operatorname{Re}\left(\lambda_{2,+}-\lambda_{3,-}\right)<0 & \text { on } i \mathbb{R} \backslash S\left(\sigma_{2}-\mu_{2}\right), \\
\operatorname{Re}\left(\lambda_{4,+}-\lambda_{3,-}\right)<0 & \text { on } \mathbb{R} \backslash S\left(\mu_{3}\right) .
\end{array}
$$

Proof. The inequalities follow from the inequalities in Lemma 4.4.

For example, to obtain (4.43) we note that by (4.35) and (2.14)

$$
\begin{aligned}
\lambda_{2,+}-\lambda_{1,-} & =\left(g_{1}-g_{2}+\theta_{1}\right)_{+}-\left(V-g_{1}-\ell_{1}\right)_{-} \\
& =g_{1,+}+g_{1,-}-g_{2,+}-V_{1}+\ell_{1}
\end{aligned}
$$

which indeed has a negative real part on $\mathbb{R} \backslash S\left(\mu_{1}\right)$ because of the inequality in (4.10). The inequality is strict because of the regularity assumption.

The other inequalities (4.44) and (4.45) follow in a similar way.

The asymptotic behavior of the $\lambda$-functions follows by combining the definition (4.35) with the asymptotics of the $g$-function, which we state for ease of future reference.

Lemma 4.14. We have as $z \rightarrow \infty$

$$
\begin{aligned}
& \lambda_{1}(z)=V(z)-\log z-\ell_{1}+\mathcal{O}\left(z^{-1}\right), \\
& \lambda_{2}(z)=\theta_{1}(z)+ \begin{cases}\frac{1}{3} \log z-\frac{3}{2} C z^{-2 / 3}+\mathcal{O}\left(z^{-1}\right), & z \in I \cup I V, \\
\frac{1}{3} \log z-\frac{3}{2} C \omega z^{-2 / 3}+\mathcal{O}\left(z^{-1}\right), & z \in I I, \\
\frac{1}{3} \log z-\frac{3}{2} C \omega^{2} z^{-2 / 3}+\mathcal{O}\left(z^{-1}\right), & z \in I I I,\end{cases} \\
& \lambda_{3}(z)=\theta_{2}(z)+ \begin{cases}\frac{1}{3} \log z-\frac{3}{2} C \omega z^{-2 / 3}+\mathcal{O}\left(z^{-1}\right), & z \in I, \\
\frac{1}{3} \log z-\frac{3}{2} C z^{-2 / 3}+\mathcal{O}\left(z^{-1}\right), & z \in I I \cup I I I, \\
\frac{1}{3} \log z-\frac{3}{2} C \omega^{2} z^{-2 / 3}+\mathcal{O}\left(z^{-1}\right), & z \in I V,\end{cases} \\
& \lambda_{4}(z)=\theta_{3}(z)+ \begin{cases}\frac{1}{3} \log z-\frac{3}{2} C \omega^{2} z^{-2 / 3}+\mathcal{O}\left(z^{-1}\right), & z \in I \cup I I, \\
\frac{1}{3} \log z-\frac{3}{2} C \omega z^{-2 / 3}+\mathcal{O}\left(z^{-1}\right), & z \in I I I \cup I V,\end{cases}
\end{aligned}
$$

where $C$ is the constant from (4.31). 


\section{CHAPTER 5}

\section{Pearcey Integrals and the First Transformation}

Now we come to the first transformation of the RH problem (1.36) which as in 45. will be done with the help of Pearcey integrals. The present setup is however slightly different from the one in $\mathbf{4 5}$, since we will construct a $\mathrm{RH}$ problem for the Pearcey integrals that is $n$-dependent.

\subsection{Definitions}

We note that the weights from (1.35) can be written as

$$
\left\{\begin{array}{l}
w_{0, n}(x)=e^{-n V(x)} p_{0, n}(x), \\
w_{1, n}(x)=(n \tau)^{-1} e^{-n V(x)} p_{0, n}^{\prime}(x), \\
w_{2, n}(x)=(n \tau)^{-2} e^{-n V(x)} p_{0, n}^{\prime \prime}(x),
\end{array}\right.
$$

where

$$
p_{0, n}(x)=\int_{-\infty}^{\infty} e^{-n(W(s)-\tau x s)} d s, \quad W(s)=\frac{1}{4} s^{4}+\frac{\alpha}{2} s^{2},
$$

is a Pearcey integral that satisfies the third order linear ODE

$$
p^{\prime \prime \prime}+n^{2} \tau^{2} \alpha p^{\prime}-n^{3} \tau^{4} x p=0 .
$$

Other solutions to the same ODE are given by similar integrals.

Definition 5.1. For $j=0, \ldots, 5$ and $n \in \mathbb{N}$, we define

$$
p_{j, n}(z)=\int_{\Gamma_{j}} e^{-n(W(s)-\tau z s)} d s, \quad z \in \mathbb{C},
$$

where the contours $\Gamma_{j}$ are

$$
\begin{cases}\Gamma_{0}=(-\infty, \infty), & \Gamma_{1}=(i \infty, 0] \cup[0, \infty), \\ \Gamma_{2}=(i \infty, 0] \cup[0,-\infty), & \Gamma_{3}=(-i \infty, 0] \cup[0,-\infty), \\ \Gamma_{4}=(-i \infty, 0] \cup[0, \infty), & \Gamma_{5}=i \mathbb{R},\end{cases}
$$

or homotopic deformations such as the ones shown in Figure [5.1, and with the orientation as also shown in Figure 5.1

All Pearcey integrals (5.4) are entire functions in the complex plane.

Certain combinations of these functions are used to build a $3 \times 3$ matrix valued $P_{n}: \mathbb{C} \backslash(\mathbb{R} \cup i \mathbb{R}) \rightarrow \mathbb{C}^{3 \times 3}$ as follows. 


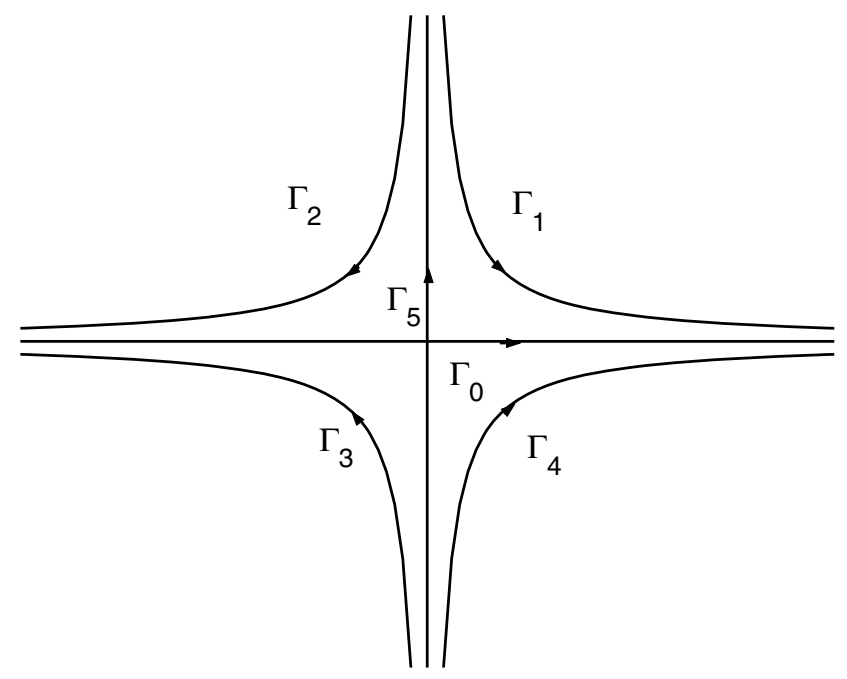

Figure 5.1. Contours $\Gamma_{j}$ in the definition of the Pearcey integrals

DEFINITION 5.2. In each of the four quadrants (denoted $I, I I, I I I$ and $I V$ ) we define

$$
P_{n}=\left\{\begin{array}{lll}
\left(\begin{array}{lll}
p_{0, n} & -p_{2, n} & -p_{5, n} \\
p_{0, n}^{\prime} & -p_{2, n}^{\prime} & -p_{5, n}^{\prime} \\
p_{0, n}^{\prime \prime} & -p_{2, n}^{\prime \prime} & -p_{5, n}^{\prime \prime}
\end{array}\right) & \text { in } I, \\
\left(\begin{array}{lll}
p_{0, n} & -p_{1, n} & -p_{5, n} \\
p_{0, n}^{\prime} & -p_{1, n}^{\prime} & -p_{5, n}^{\prime} \\
p_{0, n}^{\prime \prime} & -p_{1, n}^{\prime \prime} & -p_{5, n}^{\prime \prime}
\end{array}\right) & \text { in } I I, \\
\left(\begin{array}{lll}
p_{0, n} & -p_{4, n} & -p_{5, n} \\
p_{0, n}^{\prime} & -p_{4, n}^{\prime} & -p_{5, n}^{\prime} \\
p_{0, n}^{\prime \prime} & -p_{4, n}^{\prime \prime} & -p_{5, n}^{\prime \prime}
\end{array}\right) & \text { in } I I I, \\
\left(\begin{array}{lll}
p_{0, n} & -p_{3, n} & -p_{5, n} \\
p_{0, n}^{\prime} & -p_{3, n}^{\prime} & -p_{5, n}^{\prime} \\
p_{0, n}^{\prime \prime} & -p_{3, n}^{\prime \prime} & -p_{5, n}^{\prime \prime}
\end{array}\right) & \text { in } I V .
\end{array}\right.
$$

Then $P_{n}$ is indeed defined and analytic in $\mathbb{C} \backslash(\mathbb{R} \cup i \mathbb{R})$ with the following jump properties on the real line (oriented from left to right) and the imaginary axis (oriented from bottom to top). As usual the orientation determines the + and side of the curve, where the + side is on the left and the - side on the right.

LEMMA 5.3. The matrix valued function $P_{n}$ satisfies the jump relations

$$
\begin{array}{ll}
P_{n,+}(z)=P_{n,-}(z)\left(\begin{array}{ccc}
1 & 0 & 0 \\
0 & 1 & 0 \\
0 & -1 & 1
\end{array}\right), & z \in \mathbb{R}, \\
P_{n,+}(z)=P_{n,-}(z)\left(\begin{array}{ccc}
1 & -1 & 0 \\
0 & 1 & 0 \\
0 & 0 & 1
\end{array}\right), & z \in i \mathbb{R} .
\end{array}
$$


Proof. The jumps easily follow from the definitions. It follows for example from the definition of the Pearcey integrals and the orientation of the contours $\Gamma_{j}$, see Figure 5.1, that

$$
-p_{2, n}=-p_{3, n}+p_{5, n} .
$$

This relation implies the jump relation (5.7) for $z \in \mathbb{R}^{+}$in view of the definition of $P_{n}$ in the first and fourth quadrant given in (5.6).

The other jumps are proved in a similar way.

\subsection{Large $z$ asymptotics}

The large $z$ behavior of $P_{n}(z)$ is obtained from a classical saddle point analysis of the integrals (5.4) that defines each of its entries. Recall that the saddle point equation (2.2) has the three solutions $s_{1}(z), s_{2}(z)$ and $s_{3}(z)$, see Section 2.1. The value of $-W(s)+\tau z s$ at the saddle $s_{j}(z)$ is denoted by $\theta_{j}(z)$, as in Section 2.2 .

LEMmA 5.4. We have as $z \rightarrow \infty$,

$$
\begin{aligned}
& P_{n}(z)=\sqrt{\frac{2 \pi n}{3}} \tau\left(\begin{array}{ccc}
n^{-1} \tau^{-4 / 3} z^{-1 / 3} & 0 & 0 \\
0 & 1 & 0 \\
0 & 0 & n \tau^{4 / 3} z^{1 / 3}
\end{array}\right) \\
& \times\left(I+\frac{\alpha}{6}(\tau z)^{-2 / 3}\left(\begin{array}{ccc}
0 & 1 & 0 \\
0 & 0 & -1 \\
-3 & 0 & 0
\end{array}\right)+\mathcal{O}\left(z^{-4 / 3}\right)\right)
\end{aligned}
$$

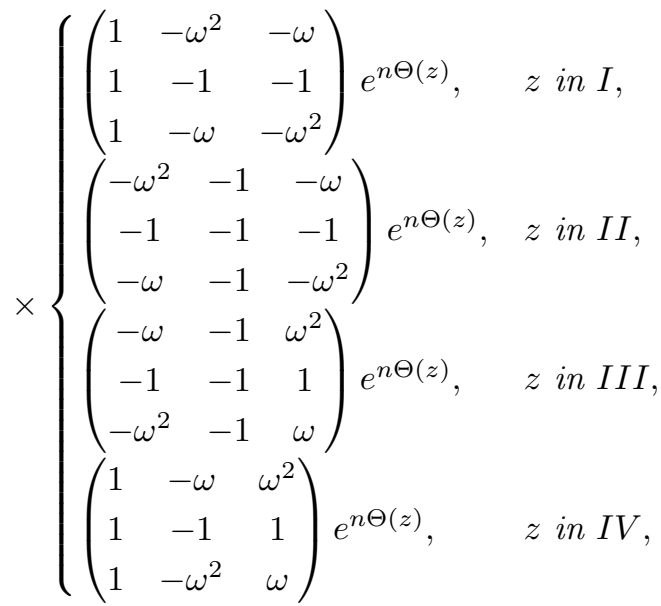

where $\Theta$ is defined as in (2.11).

Proof. The proof is a tedious saddle point analysis for all integrals that define $P_{n}$ in the respective quadrants, see also 21. The ODE (5.3) can be used to find the form of the asymptotic expansion. Indeed, putting $p=e^{n F}$ in (5.3) we obtain the following differential equation for $f=F^{\prime}$,

$$
f^{3}-\tau^{4} z+\tau^{2} \alpha f+\frac{3}{n} f f^{\prime}+\frac{1}{n^{2}} f^{\prime \prime}=0 .
$$

This nonlinear ODE has solutions $f$ with expansions

$$
f(z)=\omega^{k} \tau^{4 / 3} z^{1 / 3}-\frac{\alpha}{3} \omega^{2 k} \tau^{2 / 3} z^{-1 / 3}-\frac{1}{3 n} z^{-1}
$$




$$
+\left(\frac{\alpha^{3}}{81}-\frac{\alpha}{9 n}\right) \omega^{k} \tau^{-2 / 3} z^{-5 / 3}+\mathcal{O}\left(z^{-7 / 3}\right), \quad k=0,1,2,
$$

as $z \rightarrow \infty$ in one of the quadrants. Here $\omega=e^{2 \pi i / 3}$, as before. Thus after integration and by Proposition 2.4, we have for a certain $j=1,2,3$,

$$
F(z)=\theta_{j}(z)-\frac{1}{3 n} \log z+\frac{1}{n} \log C+\frac{\alpha}{6 n} \omega^{k}(\tau z)^{-2 / 3}+\mathcal{O}\left(z^{-4 / 3}\right)
$$

where $C$ is a constant, and so there are solutions of the Pearcey ODE (5.3) that behave like

$$
p(z)=C z^{-1 / 3} e^{n \theta_{j}(z)}\left(1+\frac{\alpha}{6} \omega^{k}(\tau z)^{-2 / 3}+\mathcal{O}\left(z^{-4 / 3}\right)\right)
$$

as $z \rightarrow \infty$ in one of the quadrants.

Each of the functions $p_{0, n}, \ldots, p_{5, n}$ that appears in the definition of $P_{n}$ in a certain quadrant has an asymptotic expansion as in (5.10). We have to associate with each such function a value of $j$ and the corresponding value of $k$. To do this, we have to perform a saddle point analysis on the integral representation (5.4).

The saddle point equation

$$
W^{\prime}(s)-\tau z=s^{3}+\alpha s-\tau z=0
$$

for (5.4) has three solutions $s_{j}(z)$. It turns out that in each quadrant and for each $j=1,2,3$, we have that $s_{j}(z)$ is the relevant saddle for the Pearcey integral that defines the function in the $j$ th column. Thus the initial contour can be deformed into the steepest descent path through $s_{j}(z)$, or into a union of steepest descent paths with $s_{j}(z)$ as the determining saddle.

Then by a classical saddle point analysis, see e.g. 74, we obtain the following asymptotic behavior

$$
\begin{aligned}
p(z) & = \pm \sqrt{\frac{2 \pi}{n W^{\prime \prime}\left(s_{j}(z)\right)}} e^{-n\left(W\left(s_{j}(z)\right)-\tau z s_{j}(z)\right)}\left(1+\mathcal{O}\left(z^{-2 / 3}\right)\right) \\
& = \pm \sqrt{\frac{2 \pi}{n W^{\prime \prime}\left(s_{j}(z)\right)}} e^{n \theta_{j}(z)}\left(1+\mathcal{O}\left(z^{-2 / 3}\right)\right)
\end{aligned}
$$

as $z \rightarrow \infty$. Since $W^{\prime \prime}(s)=3 s^{2}+\alpha$, and $s_{j}(z)=\mathcal{O}\left(z^{1 / 3}\right)$ by Lemma 2.3, we find from (5.12) the behavior

$$
p(z)= \pm \sqrt{\frac{2 \pi}{3 n}} s_{j}(z)^{-1} e^{n \theta_{j}(z)}\left(1+\mathcal{O}\left(z^{-2 / 3}\right)\right)
$$

which then by (5.10) takes the form

$$
p(z)= \pm \sqrt{\frac{2 \pi}{3 n}} \omega^{2 k}(\tau z)^{-1 / 3} e^{n \theta_{j}(z)}\left(1+\frac{\alpha}{6} \omega^{k}(\tau z)^{-2 / 3}+\mathcal{O}\left(z^{-4 / 3}\right)\right)
$$

for a certain value of $k$, depending on $j$ and depending on the particular quadrant. In the first quadrant, for example, we have $k=j-1$ as follows from Lemma 2.3.

We may differentiate the expansions and we obtain for the derivative

$$
\begin{aligned}
p^{\prime}(z) & = \pm \sqrt{\frac{2 \pi}{3 n}} \omega^{2 k}(\tau z)^{-1 / 3} n \tau s_{j}(z) e^{n \theta_{j}(z)}\left(1+\frac{\alpha}{6} \omega^{k}(\tau z)^{-2 / 3}+\mathcal{O}\left(z^{-4 / 3}\right)\right) \\
& = \pm \sqrt{\frac{2 \pi}{3 n}} n \tau e^{n \theta_{j}(z)}\left(1-\frac{\alpha}{6} \omega^{k}(\tau z)^{-2 / 3}+\mathcal{O}\left(z^{-4 / 3}\right)\right)
\end{aligned}
$$


and for the second derivative

$$
p^{\prime \prime}(z)= \pm \sqrt{\frac{2 \pi}{3 n}}(n \tau)^{2} \omega^{k}(\tau z)^{1 / 3} e^{n \theta_{j}(z)}\left(1-\frac{\alpha}{2} \omega^{k}(\tau z)^{-2 / 3}+\mathcal{O}\left(z^{-4 / 3}\right)\right) .
$$

In each quadrant we have to take the correct sign (determined by the orientation of the contours) and the correct value of $k$. The formulas can then be written in the form (5.9).

Let us define the constant matrices $A_{j}$ as follows.

Definition 5.5. We define

$$
\begin{array}{ll}
A_{1}=\frac{i}{\sqrt{3}}\left(\begin{array}{ccc}
1 & -\omega & -\omega^{2} \\
1 & -1 & -1 \\
1 & -\omega^{2} & -\omega
\end{array}\right), & A_{2}=\frac{i}{\sqrt{3}}\left(\begin{array}{ccc}
-\omega & -1 & -\omega^{2} \\
-1 & -1 & -1 \\
-\omega^{2} & -1 & -\omega
\end{array}\right), \\
A_{3}=\frac{i}{\sqrt{3}}\left(\begin{array}{ccc}
-\omega^{2} & -1 & \omega \\
-1 & -1 & 1 \\
-\omega & -1 & \omega^{2}
\end{array}\right), & A_{4}=\frac{i}{\sqrt{3}}\left(\begin{array}{ccc}
1 & -\omega^{2} & \omega \\
1 & -1 & 1 \\
1 & -\omega & \omega^{2}
\end{array}\right) .
\end{array}
$$

The prefactor $\frac{i}{\sqrt{3}}$ is chosen such that $\operatorname{det} A_{j}=1$ for $j=1,2,3,4$. Then we can reformulate Lemma 5.4 as follows.

Corollary 5.6. We have as $z \rightarrow \infty$ in the jth quadrant,

$$
P_{n}(z)=P_{n, 0}\left(I+\mathcal{O}\left(z^{-2 / 3}\right)\right) \operatorname{diag}\left(z^{-1 / 3} \quad 1 \quad z^{1 / 3}\right) A_{j}^{-t} e^{n \Theta(z)}
$$

where $P_{n, 0}$ is the invertible matrix

$$
P_{n, 0}=\sqrt{2 \pi n} \tau i\left(\begin{array}{ccc}
n^{-1} \tau^{-4 / 3} & 0 & 0 \\
0 & 1 & 0 \\
-\frac{\alpha}{2} n \tau^{2 / 3} & 0 & n \tau^{4 / 3}
\end{array}\right)
$$

The asymptotic formula (5.15) will be the most convenient to work with in what follows. Note that we still have an error term $I+\mathcal{O}\left(z^{-2 / 3}\right)$ which is somewhat remarkable.

\subsection{First transformation: $Y \mapsto X$}

With the $3 \times 3$ matrix valued $P_{n}$ we can now perform the first transformation of the Riemann-Hilbert problem. Recall that $Y$ is the solution of the $\mathrm{RH}$ problem (1.36).

Definition 5.7. We define $X$ by

$$
X(z)=\left(\begin{array}{cc}
1 & 0 \\
0 & C_{n}
\end{array}\right) Y(z)\left(\begin{array}{cc}
1 & 0 \\
0 & D_{n} P_{n}^{-t}(z) e^{n \Theta(z)}
\end{array}\right),
$$

for $z$ in the $j$ th quadrant. Here $C_{n}$ and $D_{n}$ are the constant matrices

$$
C_{n}=P_{n, 0}^{t} D_{n}^{-1}, \quad D_{n}=\operatorname{diag}\left(\begin{array}{lll}
1 & n \tau & (n \tau)^{2}
\end{array}\right),
$$

with $P_{n, 0}$ given by (5.16), and $P_{n}$ is given by (5.6), and $\Theta$ is given by (2.11).

The matrices in the right-hand side of (5.17) are $4 \times 4$ matrices written in block form, where the right lower block has size $3 \times 3$. The transformation (5.17) does not affect the $(1,1)$ entry. The factor $e^{n \Theta}(z)$ is included in the definition of $X(z)$ in order to simplify the asymptotic behavior of $X$. However, it will complicate the jump matrices, as we will see. 
The matrix valued function $X$ is defined and analytic in each of the four quadrants.

The asymptotic behavior for $X$ is as follows.

LEMMA 5.8. We have

$$
X(z)=\left(I+\mathcal{O}\left(z^{-2 / 3}\right)\right)\left(\begin{array}{cccc}
z^{n} & 0 & 0 & 0 \\
0 & z^{-\frac{n}{3}+\frac{1}{3}} & 0 & 0 \\
0 & 0 & z^{-\frac{n}{3}} & 0 \\
0 & 0 & 0 & z^{-\frac{n}{3}-\frac{1}{3}}
\end{array}\right)\left(\begin{array}{cc}
1 & 0 \\
0 & A_{j}
\end{array}\right)
$$

as $z \rightarrow \infty$ in the $j$ th quadrant with matrices where the matrices $A_{j}$ are given by (5.13) and (5.14).

As in 45 we have that the asymptotic formula (5.19) for $X$ is not uniform up to the axis.

Proof. By Corollary [5.6] and the definitions (5.18) of $C_{n}$ and $D_{n}$ we have

$$
C_{n} D_{n} P_{n}^{-t}(z) e^{n \Theta(z)}=\left(I+\mathcal{O}\left(z^{-2 / 3}\right)\right)\left(\begin{array}{ccc}
z^{1 / 3} & 0 & 0 \\
0 & 1 & 0 \\
0 & 0 & z^{-1 / 3}
\end{array}\right) A_{j}
$$

as $z \rightarrow \infty$ in the $j$ th quadrant. Using this in (5.17), together with the asymptotics of $Y$ as given in (1.36), we obtain the lemma.

The jumps for $X$ on the real and imaginary axis are as follows.

LEMMA 5.9. We have

$$
X_{+}(z)=X_{-}(z) J_{X}(z), \quad z \in \mathbb{R} \cup i \mathbb{R},
$$

where the jump matrices $J_{X}$ are given as follows.

- On the real line we have for $x \in\left(-\infty,-x^{*}(\alpha)\right] \cup\left[x^{*}(\alpha), \infty\right)$

$$
J_{X}(x)=\left(\begin{array}{cccc}
1 & e^{-n\left(V(x)-\theta_{1}(x)\right)} & 0 & 0 \\
0 & 1 & 0 & 0 \\
0 & 0 & e^{n\left(\theta_{2,+}(x)-\theta_{3,+}(x)\right)} & 1 \\
0 & 0 & 0 & e^{n\left(\theta_{3,+}(x)-\theta_{2,+}(x)\right)}
\end{array}\right)
$$

and for $x \in\left(-x^{*}(\alpha), x^{*}(\alpha)\right)$ (only relevant in case $\left.\alpha<0\right)$,

$$
J_{X}(x)=\left(\begin{array}{cccc}
1 & e^{-n\left(V(x)-\theta_{1}(x)\right)} & 0 & 0 \\
0 & 1 & 0 & 0 \\
0 & 0 & 1 & e^{-n\left(\theta_{2}(x)-\theta_{3}(x)\right)} \\
0 & 0 & 0 & 1
\end{array}\right)
$$

- On the imaginary axis, we have for $z \in\left(-i \infty,-i y^{*}(\alpha)\right] \cup\left[i y^{*}(\alpha), i \infty\right)$,

$$
J_{X}(z)=\left(\begin{array}{cccc}
1 & 0 & 0 & 0 \\
0 & e^{n\left(\theta_{1,+}(z)-\theta_{2,+}(z)\right)} & 0 & 0 \\
0 & 1 & e^{n\left(\theta_{2,+}(z)-\theta_{1,+}(z)\right)} & 0 \\
0 & 0 & 0 & 1
\end{array}\right),
$$


and for $z \in\left(-i y^{*}(\alpha), i y^{*}(\alpha)\right)$ (only relevant in case $\alpha>0$ ),

$$
J_{X}(z)=\left(\begin{array}{cccc}
1 & 0 & 0 & 0 \\
0 & 1 & 0 & 0 \\
0 & e^{-n\left(\theta_{2}(z)-\theta_{1}(z)\right)} & 1 & 0 \\
0 & 0 & 0 & 1
\end{array}\right) .
$$

Proof. The jump matrix for $x \in \mathbb{R}$ is by (5.17) and the jump condition in (1.36)

$$
\begin{aligned}
& J_{X}(x)=\left(\begin{array}{cc}
1 & 0 \\
0 & e^{-n \Theta_{-}(x)} P_{n,-}^{t}(x) D_{n}^{-1}
\end{array}\right)\left(\begin{array}{cccc}
1 & w_{0, n}(x) & w_{1, n}(x) & w_{2, n}(x) \\
0 & 1 & 0 & 0 \\
0 & 0 & 1 & 0 \\
0 & 0 & 0 & 1
\end{array}\right) \\
& \times\left(\begin{array}{cc}
1 & 0 \\
0 & D_{n} P_{n,+}^{-t}(x) e^{n \Theta_{+}(x)}
\end{array}\right) \\
& =\left(\begin{array}{cccc}
1 & \left(w_{0, n}(x)\right. & w_{1, n}(x) & \left.w_{2, n}(x)\right) D_{n} P_{n,+}^{-t}(x) e^{n \Theta_{+}(x)} \\
0 & e^{-n \Theta_{-}(x)} P_{n,-}^{t}(x) P_{n,+}^{-t}(x) e^{n \Theta_{+}(x)}
\end{array}\right)
\end{aligned}
$$

The row vector in the right upper corner of (5.24) is by (5.1) and (5.18)

$$
\begin{aligned}
\left(w_{0, n}(x) \quad w_{1, n}(x)\right. & \left.w_{2, n}(x)\right) D_{n} P_{n,+}^{-t}(x) e^{n \Theta_{+}(x)} \\
= & e^{-n V(x)}\left(p_{0, n}(x) \quad p_{0, n}^{\prime}(x) \quad p_{0, n}^{\prime \prime}(x)\right) P_{n,+}^{-t}(x) e^{n \Theta_{+}(x)}
\end{aligned}
$$

Since $\left(p_{0, n}(x) \quad p_{0, n}^{\prime}(x) \quad p_{0, n}^{\prime \prime}(x)\right)^{t}$ is the first column of $P_{n}(x)$, see (5.6), it follows that (5.25) is equal to $e^{-n V(x)}\left(\begin{array}{lll}1 & 0 & 0\end{array}\right) e^{n \Theta_{+}(x)}$ which by (2.11) leads to

$$
\begin{aligned}
\left(w_{0, n}(x) \quad w_{1, n}(x)\right. & \left.w_{2, n}(x)\right) D_{n} P_{n,+}^{-t}(x) e^{n \Theta_{+}(x)} \\
& =\left(\begin{array}{lll}
e^{-n\left(V(x)-\theta_{1}(x)\right)} & 0 & 0
\end{array}\right) .
\end{aligned}
$$

This leads to the first row of the jump matrices (5.20)-(5.21).

To evaluate the $3 \times 3$ block in the right lower corner of (5.24) we note that we have by (5.7)

$$
P_{n,-}^{t}(x) P_{n,+}^{-t}(x)=\left(P_{n,-}^{-1}(x) P_{n,+}(x)\right)^{-t}=\left(\begin{array}{ccc}
1 & 0 & 0 \\
0 & 1 & 1 \\
0 & 0 & 1
\end{array}\right)
$$

so that

$$
e^{-n \Theta_{-}(x)} P_{n,-}^{t}(x) P_{n,+}^{-t}(x) e^{n \Theta_{+}(x)}=e^{-n \Theta_{-}(x)}\left(\begin{array}{ccc}
1 & 0 & 0 \\
0 & 1 & 1 \\
0 & 0 & 1
\end{array}\right) e^{n \Theta_{+}(x)}
$$

Then using (2.12) to write $e^{-n \Theta_{-}(x)}$ in terms of $e^{-n \Theta_{+}(x)}$, and then using the explicit expressions (2.11) for $\Theta$, we see that (5.27) indeed reduces to the $3 \times 3$ right lower block in (5.20)-(5.21).

The proof of the other expressions for $J_{X}$ follows in a similar way. 


\subsection{RH problem for $X$}

To summarize, we have found the following RH problem for $X$.

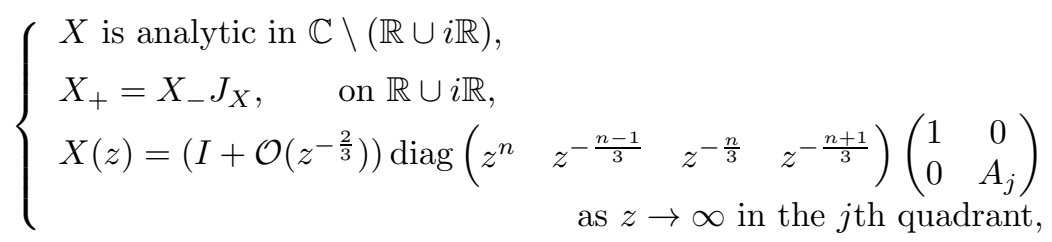

where $J_{X}$ is given by (5.20)-(5.23).

Each of the jump matrices $J_{X}$ is nontrivial only in certain $2 \times 2$ blocks. The nontrivial blocks are triangular and assume one of the forms

$$
\left(\begin{array}{ll}
1 & * \\
0 & 1
\end{array}\right) \text { or }\left(\begin{array}{ll}
1 & 0 \\
* & 1
\end{array}\right)
$$

with a real off-diagonal entry $*$, or

$$
\left(\begin{array}{ll}
* & 1 \\
0 & *
\end{array}\right) \text { or }\left(\begin{array}{ll}
* & 0 \\
1 & *
\end{array}\right)
$$

with oscillatory diagonal entries of absolute value 1 . The first form indicates that an external field is acting and the second form indicates the presence of an upper constraint. In this way we can already see the connection with the vector equilibrium problem.

Let us examine this in more detail.

Jump $J_{X}$ on the real line. The jump matrix $J_{X}$ on the real line, see (5.20) and (5.21), takes the block form

$$
J_{X}=\left(\begin{array}{cc}
\left(J_{X}\right)_{1} & 0 \\
0 & \left(J_{X}\right)_{3}
\end{array}\right), \quad \text { on } \mathbb{R},
$$

where $\left(J_{X}\right)_{1}$ and $\left(J_{X}\right)_{3}$ are $2 \times 2$ matrices.

We have

$$
\left(J_{X}\right)_{1}(x)=\left(\begin{array}{cc}
1 & e^{-n V_{1}(x)} \\
0 & 1
\end{array}\right), \quad x \in \mathbb{R}
$$

where

$$
V_{1}(x)=V(x)-\theta_{1}(x)
$$

is indeed the external field that acts on the first measure in the vector equilibrium problem, see (2.14).

Furthermore, we have by (5.21),

$$
\left(J_{X}\right)_{3}(x)=\left(\begin{array}{cc}
1 & e^{-n V_{3}(x)} \\
0 & 1
\end{array}\right), \quad x \in\left(-x^{*}(\alpha), x^{*}(\alpha)\right)
$$

with

$$
V_{3}(x)=\theta_{2}(x)-\theta_{3}(x) \quad \text { for } x \in\left(-x^{*}(\alpha), x^{*}(\alpha)\right),
$$

which by (2.15) is indeed the non-zero part of the external field $V_{3}$ that acts on the third measure in the vector equilibrium problem. The external field $V_{3}$ plays a role only in case $x^{*}(\alpha)>0$, that is, in case $\alpha<0$. 


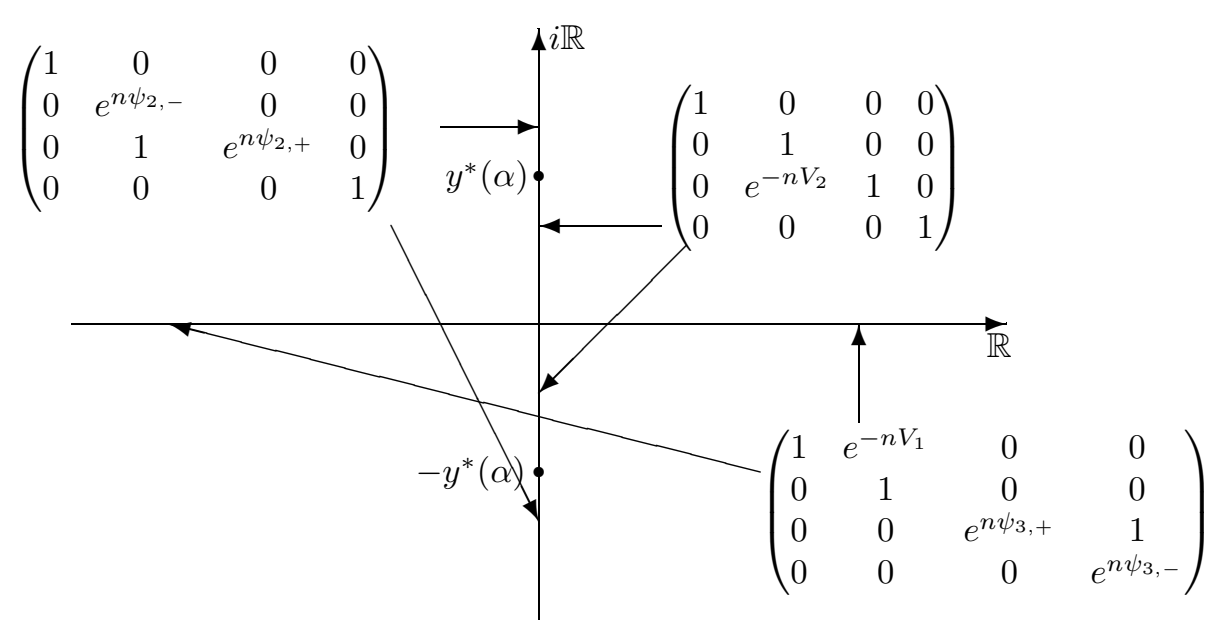

FiguRE 5.2. Jump matrices $J_{X}$ in case $\alpha>0$

The right lower block in (5.20) has oscillatory diagonal entries. We define $\psi_{3}$ by

$$
\psi_{3}(z)=\theta_{2}(z)-\theta_{3}(z)
$$

so that

$$
\left(J_{X}\right)_{3}(x)=\left(\begin{array}{cc}
e^{n \psi_{3,+}(x)} & 1 \\
0 & e^{n \psi_{3,-}(x)}
\end{array}\right), \quad x \in \mathbb{R},|x|>x^{*}(\alpha) .
$$

Then $\psi_{3, \pm}$ is purely imaginary for $|x|>x^{*}(\alpha)$ with

$$
\begin{aligned}
\frac{d}{d x} \psi_{3, \pm}(x) & =\frac{d}{d x}\left[\theta_{2,+}(x)-\theta_{2,-}(x)\right] \\
& =\tau\left(s_{2,+}(x)-s_{2,-}(x)\right)=2 i \tau \operatorname{Im} s_{2,+}(x),
\end{aligned}
$$

which is purely imaginary with positive imaginary part. Thus we can associate with $\psi_{3}$ a measure $\sigma_{3}$ on $\mathbb{R}$ by putting

$$
\frac{d \sigma_{3}}{d x}=\frac{1}{2 \pi i} \frac{d}{d x}\left(\theta_{2,+}(x)-\theta_{3,+}(x)\right)=\frac{\tau}{\pi} \operatorname{Im} s_{2,+}(x), \quad \text { for } x \in \mathbb{R} .
$$

Then $S\left(\sigma_{3}\right)=\mathbb{R}$ if $\alpha>0$ and $S\left(\sigma_{3}\right)=\mathbb{R} \backslash\left(-x^{*}(\alpha), x^{*}(\alpha)\right)$ if $\alpha<0$.

Because of the upper triangular form of (5.32) it will turn out that $\sigma_{3}$ acts as a lower constraint on the third measure in the sense that

$$
\mu_{3}+\sigma_{3} \geq 0
$$

and then we could allow signed measures $\mu_{3}$ in the vector equilibrium problem. However, in this more general vector equilibrium problem we would still find $\mu_{3} \geq 0$ so that the constraint (5.34) does not play a role after all.

We will not use the measure $\sigma_{3}$ anymore. 


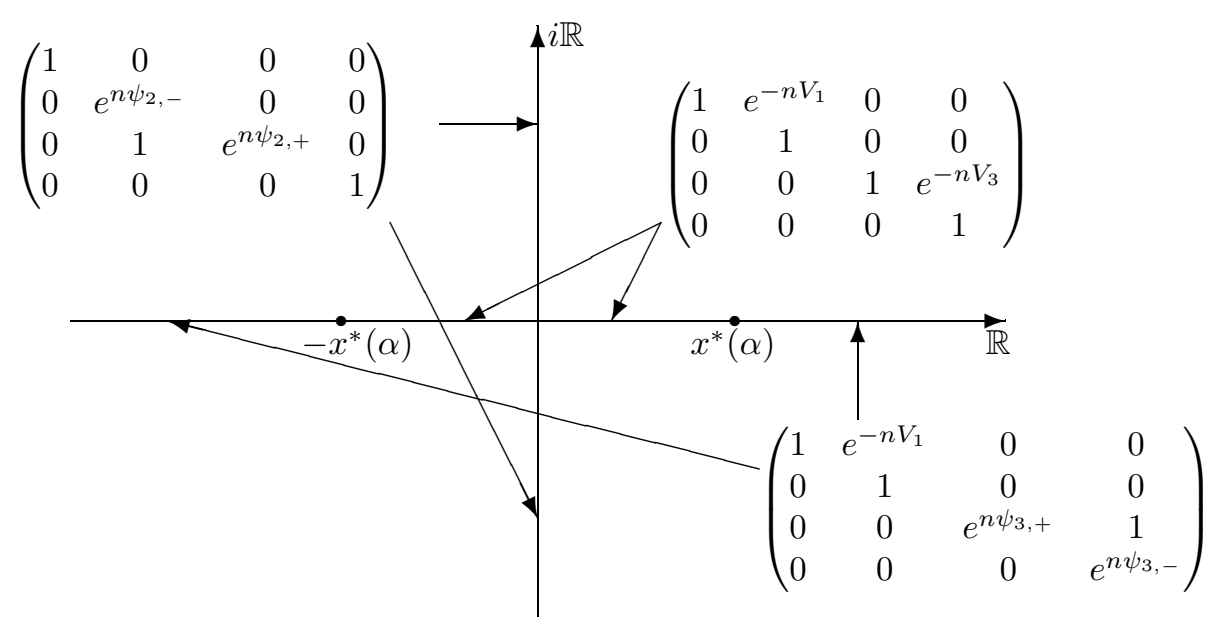

FiguRE 5.3. Jump matrices $J_{X}$ in case $\alpha<0$

Jump $J_{X}$ on the imaginary axis. The jump matrix $J_{X}$ on the imaginary axis, see (5.22) and (5.23), takes the block form

$$
J_{X}=\left(\begin{array}{ccc}
1 & 0 & 0 \\
0 & \left(J_{X}\right)_{2} & 0 \\
0 & 0 & 1
\end{array}\right), \quad \text { on } i \mathbb{R}
$$

where $\left(J_{X}\right)_{2}$ is a $2 \times 2$ matrix.

We define

$$
\psi_{2}(z)=\theta_{1}(z)-\theta_{2}(z), \quad z \in i \mathbb{R},|z|>y^{*}(\alpha),
$$

so that we have by (5.22)

$$
\left(J_{X}\right)_{2}(z)=\left(\begin{array}{cc}
e^{n \psi_{2,+}(z)} & 0 \\
1 & e^{n \psi_{2,-}(z)}
\end{array}\right), \quad z \in i \mathbb{R},|z|>y^{*}(\alpha) .
$$

Then $\psi_{2}$ is associated with the measure $\sigma_{2}$, since

$$
\begin{aligned}
\frac{d}{d z} \psi_{2,-}(z) & =\frac{d}{d z}\left[\theta_{1,-}(z)-\theta_{1,+}(z)\right] \\
& =\tau\left(s_{1,-}(z)-s_{1,+}(z)\right)=2 \pi i \frac{d \sigma_{2}}{d z}(z),
\end{aligned}
$$

by (2.7), and also

$$
\psi_{2, \pm}(z)= \begin{cases}\mp 2 \pi i \sigma_{2}([0, z]), & z \in i \mathbb{R}^{+}, \\ \mp 2 \pi i \sigma_{2}([z, 0]), & z \in i \mathbb{R}^{-} .\end{cases}
$$

We also identify an external field $V_{2}$ on the imaginary axis, which is only there in case $\alpha>0$. We have by (5.23)

$$
\left(J_{X}\right)_{2}(z)=\left(\begin{array}{cc}
1 & 0 \\
e^{-n V_{2}(z)} & 1
\end{array}\right), \quad z \in\left(-i y^{*}(\alpha), i y^{*}(\alpha)\right),
$$


with

$$
V_{2}(z)= \begin{cases}\theta_{2}(z)-\theta_{1}(z), & \text { for } z \in\left(-i y^{*}(\alpha), i y^{*}(\alpha)\right) \\ 0, & \text { elsewhere on } i \mathbb{R} .\end{cases}
$$

The external field $V_{2}$ will not be active, since it acts only on the part of $\mu_{2}$ that is in $\left(-i y^{*}(\alpha), i y^{*}(\alpha)\right)$, and this part is zero, since $S\left(\mu_{2}\right)=S\left(\sigma_{2}\right)=i \mathbb{R} \backslash$ $\left(-i y^{*}(\alpha), i y^{*}(\alpha)\right)$.

See Figures 5.2 and 5.3 for the jump matrices $J_{X}$ in the two cases $\alpha>0$ and $\alpha<0$. 


\section{CHAPTER 6 \\ Second Transformation $X \mapsto U$}

\subsection{Definition of second transformation}

The second transformation of the $\mathrm{RH}$ problem uses the functions that come from the vector equilibrium problem. It is possible to state the transformation in terms of either the $g$-functions or the $\lambda$-functions.

Definition 6.1. We define the $4 \times 4$ matrix valued function $U$ by

$$
U(z)=\left(I+\frac{3}{2} n C E_{2,4}\right) e^{n L} X(z) e^{-n G(z)},
$$

where $C$ is the constant from (4.31), $G$ is given by

$$
\begin{aligned}
G & =\operatorname{diag}\left(\begin{array}{llll}
g_{1}+\ell_{1} & g_{2}-g_{1} & g_{3}-g_{2} & -g_{3}
\end{array}\right) \\
& =-\operatorname{diag}\left(\begin{array}{llll}
\lambda_{1}-V & \lambda_{2}-\theta_{1} & \lambda_{3}-\theta_{2} & \lambda_{4}-\theta_{3}
\end{array}\right)
\end{aligned}
$$

and $L$ is a constant diagonal matrix

$$
L=\operatorname{diag}\left(\begin{array}{llll}
\ell_{1} & 0 & 0 & 0
\end{array}\right),
$$

with $\ell_{1}$ the variational constant in the Euler-Lagrange condition on $\mu_{1}$.

Note that the equality of the two diagonal matrices in (6.2) follows from the definition (4.35) of the $\lambda$-functions.

Then $U$ is defined and analytic in $\mathbb{C} \backslash(\mathbb{R} \cup i \mathbb{R})$.

\subsection{Asymptotic behavior of $U$}

LEMma 6.2. We have

$$
U(z)=\left(I+\mathcal{O}\left(z^{-1 / 3}\right)\right)\left(\begin{array}{cccc}
1 & 0 & 0 & 0 \\
0 & z^{1 / 3} & 0 & 0 \\
0 & 0 & 1 & 0 \\
0 & 0 & 0 & z^{-1 / 3}
\end{array}\right)\left(\begin{array}{cc}
1 & 0 \\
0 & A_{j}
\end{array}\right)
$$

as $z \rightarrow \infty$ in the $j$ th quadrant.

Proof. We have because of the asymptotic behavior of the $\lambda$ functions that

$$
\begin{aligned}
& e^{-n G(z)}=\operatorname{diag}\left(z^{-n} e^{-n \ell_{1}} \quad z^{n / 3} \quad z^{n / 3} \quad z^{n / 3}\right) \\
& \times\left(I-\frac{3}{2} n C z^{-2 / 3}\left(\begin{array}{cc}
0 & 0 \\
0 & \Omega_{j}
\end{array}\right)+\mathcal{O}\left(z^{-1}\right)\right)
\end{aligned}
$$


as $z \rightarrow \infty$ in the $j$ th quadrant, where

$$
\left\{\begin{array}{l}
\Omega_{1}=\operatorname{diag}\left(\begin{array}{lll}
1 & \omega & \omega^{2}
\end{array}\right) \\
\Omega_{2}=\operatorname{diag}\left(\begin{array}{lll}
\omega & 1 & \omega^{2}
\end{array}\right) \\
\Omega_{3}=\operatorname{diag}\left(\begin{array}{lll}
\omega^{2} & 1 & \omega
\end{array}\right) \\
\Omega_{4}=\operatorname{diag}\left(\begin{array}{lll}
1 & \omega^{2} & \omega
\end{array}\right)
\end{array}\right.
$$

Then by the asymptotic behavior of $X$, and the definition of $U$, we get

$$
\begin{aligned}
& U(z)=\left(I+\frac{3}{2} n C E_{2,4}+\mathcal{O}\left(z^{-2 / 3}\right)\right) \operatorname{diag}\left(\begin{array}{llll}
1 & z^{1 / 3} & 1 & z^{-1 / 3}
\end{array}\right)\left(\begin{array}{cc}
1 & 0 \\
0 & A_{j}
\end{array}\right) \\
& \times\left(I-\frac{3}{2} n C z^{-2 / 3}\left(\begin{array}{cc}
0 & 0 \\
0 & \Omega_{j}
\end{array}\right)+\mathcal{O}\left(z^{-1}\right)\right)
\end{aligned}
$$

as $z \rightarrow \infty$ in the $j$ th quadrant. We can move the $\mathcal{O}\left(z^{-1}\right)$ to the front, but then the $\mathcal{O}\left(z^{-2 / 3}\right)$ reduces to $\mathcal{O}\left(z^{-1 / 3}\right)$ :

$$
\begin{aligned}
& U(z)=\left(I+\frac{3}{2} n C E_{2,4}+\mathcal{O}\left(z^{-1 / 3}\right)\right) \operatorname{diag}\left(\begin{array}{llll}
1 & z^{1 / 3} & 1 & z^{-1 / 3}
\end{array}\right)\left(\begin{array}{cc}
1 & 0 \\
0 & A_{j}
\end{array}\right) \\
& \times\left(I-\frac{3}{2} n C z^{-2 / 3}\left(\begin{array}{cc}
0 & 0 \\
0 & \Omega_{j}
\end{array}\right)\right)
\end{aligned}
$$

We also want to move the $z^{-2 / 3}$ term to the left. Then we pick up an $\mathcal{O}(1)$ contribution in the $(2,4)$ entry. Indeed we have

$$
\begin{array}{r}
z^{-2 / 3} \operatorname{diag}\left(\begin{array}{llll}
1 & z^{1 / 3} & 1 & z^{-1 / 3}
\end{array}\right)\left(\begin{array}{cc}
1 & 0 \\
0 & A_{j} \Omega_{j} A_{j}^{-1}
\end{array}\right) \operatorname{diag}\left(\begin{array}{cccc}
1 & z^{-1 / 3} & 1 & z^{1 / 3}
\end{array}\right) \\
=E_{2,4}+\mathcal{O}\left(z^{-1 / 3}\right)
\end{array}
$$

as $z \rightarrow \infty$ in the $j$ th quadrant, since

$$
A_{j} \Omega_{j} A_{j}^{-1}=\left(\begin{array}{lll}
0 & 0 & 1 \\
1 & 0 & 0 \\
0 & 1 & 0
\end{array}\right)
$$

for every $j$. The lemma follows.

REMARK 6.3. As a consistency check, we compute the jumps of the matrix valued function $A(z)$ defined by

$$
A(z)=\operatorname{diag}\left(\begin{array}{llll}
1 & z^{1 / 3} & 1 & z^{-1 / 3}
\end{array}\right)\left(\begin{array}{cc}
1 & 0 \\
0 & A_{j}
\end{array}\right)
$$

for $z$ in the $j$ th quadrant. Then we have by the definition (5.13)-(5.14) of the matrices $A_{j}$,

$$
A_{+}(z)=A_{-}(z) J_{A}(z), \quad z \in \mathbb{R} \cup i \mathbb{R}
$$


with jump matrix

$$
J_{A}(z)=\left\{\begin{array}{l}
\left(\begin{array}{cccc}
1 & 0 & 0 & 0 \\
0 & 1 & 0 & 0 \\
0 & 0 & 0 & 1 \\
0 & 0 & -1 & 0
\end{array}\right), \\
\left(\begin{array}{cccc}
1 & 0 & 0 & 0 \\
0 & 0 & -1 & 0 \\
0 & 1 & 0 & 0 \\
0 & 0 & 0 & 1
\end{array}\right), \quad z \in \mathbb{R}, \quad z \in i \mathbb{R},
\end{array}\right.
$$

which is indeed what we expect.

\subsection{Jump matrices for $U$}

The jump $U_{+}=U_{-} J_{U}$ with jump matrix $J_{U}$ takes a different form on the various parts of the real and imaginary axis. Since $G$ and $L$ are diagonal matrices, the jump matrix $J_{U}$ has the same block structure as $J_{X}$. In terms of the $\lambda$ functions the jumps take on a very nice form.

The jump matrix $J_{U}$ on the real line has the block form

$$
J_{U}(x)=\left(\begin{array}{cc}
\left(J_{U}\right)_{1}(x) & 0 \\
0 & \left(J_{U}\right)_{3}(x)
\end{array}\right), \quad x \in \mathbb{R},
$$

with $2 \times 2$ blocks $\left(J_{U}\right)_{1}$ and $\left(J_{U}\right)_{3}$. On the imaginary axis it takes the form

$$
J_{U}(z)=\left(\begin{array}{ccc}
1 & 0 & 0 \\
0 & \left(J_{U}\right)_{2}(z) & 0 \\
0 & 0 & 1
\end{array}\right), \quad z \in i \mathbb{R}
$$

with a $2 \times 2$ block $\left(J_{U}\right)_{2}$.

LEMMA 6.4. We have

$$
\begin{aligned}
\left(J_{U}\right)_{1} & =\left(\begin{array}{cc}
e^{n\left(\lambda_{1,+}-\lambda_{1,-}\right)} & e^{n\left(\lambda_{2,+}-\lambda_{1,-}\right)} \\
0 & e^{n\left(\lambda_{2,+}-\lambda_{2,-}\right)}
\end{array}\right), \quad \text { on } \mathbb{R}, \\
\left(J_{U}\right)_{2} & =\left(\begin{array}{cc}
e^{n\left(\lambda_{2,+}-\lambda_{2,-}\right)} & 0 \\
e^{n\left(\lambda_{2,+}-\lambda_{3,-}\right)} & e^{n\left(\lambda_{3,+}-\lambda_{3,-}\right)}
\end{array}\right), \quad \text { on } i \mathbb{R},
\end{aligned}
$$

and

$$
\left(J_{U}\right)_{3}=\left(\begin{array}{cc}
e^{n\left(\lambda_{3,+}-\lambda_{3,-}\right)} & e^{n\left(\lambda_{4,+}-\lambda_{3,-}\right)} \\
0 & e^{n\left(\lambda_{4,+}-\lambda_{4,-}\right)}
\end{array}\right), \quad \text { on } \mathbb{R} .
$$

Proof. By (6.1), (6.2) we get

$$
\left(J_{U}\right)_{1}=\left(\begin{array}{cc}
e^{-n\left(\lambda_{1,-}-V\right)} & 0 \\
0 & e^{-n\left(\lambda_{2,-}-\theta_{1,-}\right)}
\end{array}\right)\left(J_{X}\right)_{1}\left(\begin{array}{cc}
e^{n\left(\lambda_{1,+}-V\right)} & 0 \\
0 & e^{n\left(\lambda_{2,+}-\theta_{1,+}\right)}
\end{array}\right) .
$$

Now $\theta_{1}$ is analytic across $\mathbb{R} \backslash\{0\}$, and $V_{1}=V-\theta_{1}$, so that by (5.29) we indeed obtain (6.9).

$$
\begin{aligned}
& \text { For }\left(J_{U}\right)_{2} \text { we find in a similar way } \\
& (6.12) \\
& \left(J_{U}\right)_{2}=\left(\begin{array}{cc}
e^{-n\left(\lambda_{2,-}-\theta_{1,-}\right)} & 0 \\
0 & e^{-n\left(\lambda_{3,-}-\theta_{2,-}\right)}
\end{array}\right)\left(J_{X}\right)_{2}\left(\begin{array}{cc}
e^{n\left(\lambda_{2,+}-\theta_{1,+}\right)} & 0 \\
0 & e^{n\left(\lambda_{3,+}-\theta_{2,+}\right)}
\end{array}\right) .
\end{aligned}
$$


56

6. SECOND TRANSFORMATION $X \mapsto U$

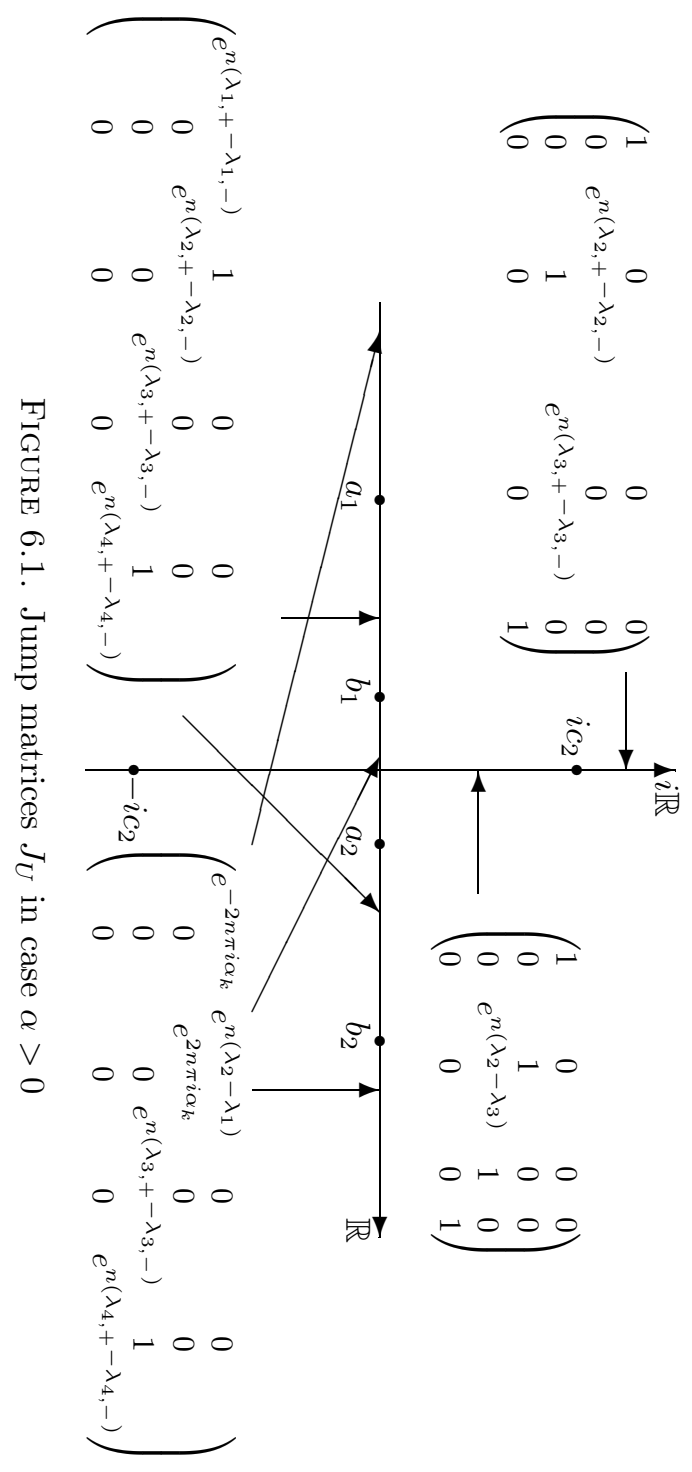

Licensed to California Institute of Technology. Prepared on Fri Aug 31 10:56:41 EDT 2012 for download from IP 131.215.220.186.

License or copyright restrictions may apply to redistribution; see http://www.ams.org/journal-terms-of-use 


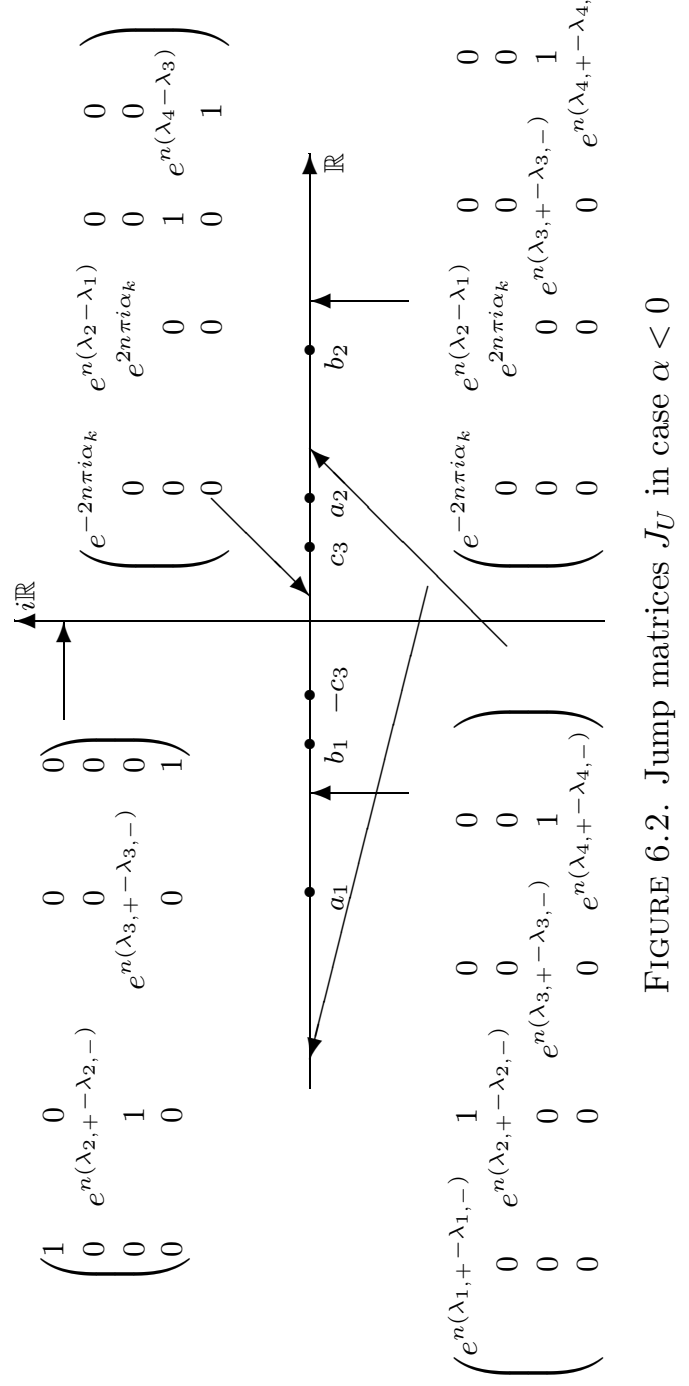


For $z \in i \mathbb{R}$ with $|z|>y^{*}(\alpha)$ we have $\theta_{1, \pm}(z)=\theta_{2, \mp}(z)$ by (2.10) and then (5.36), (5.35), and (6.12) give us that (6.10) holds on $i \mathbb{R} \backslash\left(-y^{*}(\alpha), i y^{*}(\alpha)\right)$. On $\left(-i y^{*}(\alpha), i y^{*}(\alpha)\right)$ (which is only relevant in case $\left.\alpha>0\right)$ we also obtain (6.10), but now we use the fact that $\theta_{1}$ and $\theta_{2}$ are both analytic on $\left(-i y^{*}(\alpha), i y^{*}(\alpha)\right)$, together with (5.37), (5.38), and (6.12).

For $\left(J_{U}\right)_{3}$ we obtain from (6.1) and (6.2)

$$
\left(J_{U}\right)_{3}=\left(\begin{array}{cc}
e^{-n\left(\lambda_{3,-}-\theta_{2,-}\right)} & 0 \\
0 & e^{-n\left(\lambda_{4,-}-\theta_{3,-}\right)}
\end{array}\right)\left(J_{X}\right)_{3}\left(\begin{array}{cc}
e^{n\left(\lambda_{3,+}-\theta_{2,+}\right)} & 0 \\
0 & e^{n\left(\lambda_{4,+}-\theta_{3,+}\right)}
\end{array}\right) .
$$

For $\left(J_{X}\right)_{3}$ we have the two expressions (5.30) and (5.32). Using this in (6.13) we obtain (6.11) in both cases.

The expressions in (6.9) - 6.11) are valid over the full (real or imaginary) axis. Observe in particular that the two expressions (5.36) and (5.37) for $\left(J_{X}\right)_{2}$ both lead to (6.10), and the two expressions (5.30) and (5.32) for $\left(J_{X}\right)_{3}$ both lead to (6.11). Hence the special roles that $\pm x^{*}(\alpha)$ (in case $\alpha<0$ ) and $\pm i y^{*}(\alpha)$ (in case $\alpha>0)$ played in the jump matrix $J_{X}$ for $X$ have disappeared in the jump matrix $J_{U}$ for $U$.

\subsection{RH problem for $U$}

We have found the following $\mathrm{RH}$ problem for $U$.

$$
\left\{\begin{array}{l}
U \text { is analytic in } \mathbb{C} \backslash(\mathbb{R} \cup i \mathbb{R}), \\
U_{+}=U_{-} J_{U}, \quad \text { on } \mathbb{R} \cup i \mathbb{R}, \\
U(z)=\left(I+\mathcal{O}\left(z^{-1 / 3}\right)\right) \operatorname{diag}\left(\begin{array}{llll}
1 & z^{\frac{1}{3}} & 1 & z^{-\frac{1}{3}}
\end{array}\right)\left(\begin{array}{cc}
1 & 0 \\
0 & A_{j}
\end{array}\right) \\
\text { as } z \rightarrow \infty \text { in the } j \text { th quadrant, }
\end{array}\right.
$$

where $J_{U}$ is given by (6.7)-(6.8) with $\left(J_{U}\right)_{k}$ for $k=1,2,3$ given by (6.9)-6.11).

The parts $\left(J_{U}\right)_{k}$ in the jump matrix $J_{U}$ have different expressions in the various parts of the real and imaginary axis. This follows from (6.9) -6.11) and the jump properties of the $\lambda$-functions as given in Lemma4.12. Also recall that $n$ is a multiple of three.

LEMma 6.5. (a) For $\left(J_{U}\right)_{1}$ we have

$$
\left(J_{U}\right)_{1}=\left\{\begin{array}{cc}
\left(\begin{array}{cc}
e^{n\left(\lambda_{1,+}-\lambda_{1,-}\right)} & 1 \\
0 & e^{n\left(\lambda_{2,+}-\lambda_{2,-}\right)}
\end{array}\right), & \text { on } S\left(\mu_{1}\right), \\
e^{-2 n \pi i \alpha_{k}} & e^{n\left(\lambda_{2,+}-\lambda_{1,-}\right)} \\
0 & e^{2 n \pi i \alpha_{k}}
\end{array}\right), \quad \begin{array}{ll}
\text { on }\left(b_{k}, a_{k+1}\right), \\
\text { for } k=0,1, \ldots, N .
\end{array}
$$

(b) For $\left(J_{U}\right)_{2}$ we have

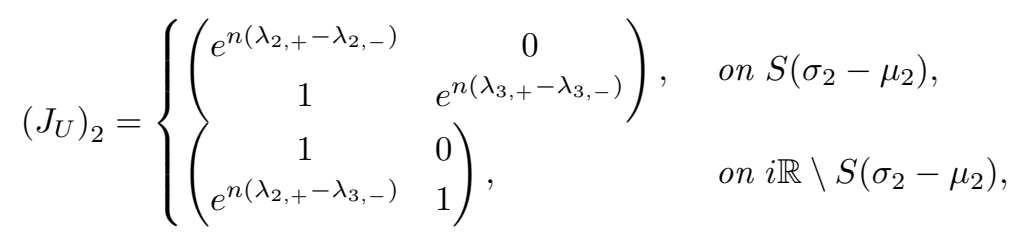


(c) For $\left(J_{U}\right)_{3}$ we have

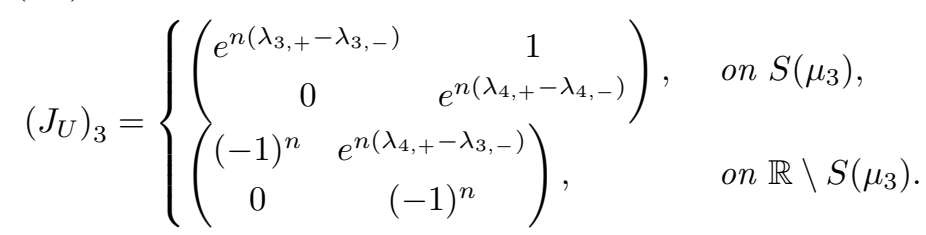

Proof. All expressions follow from (6.9) - 6.11) and Lemma 4.12,

In particular, note that on $\left(-c_{3}, c_{3}\right)$ (which can only be non-empty if $\left.\alpha<0\right)$, we have by (4.42)

$$
e^{n\left(\lambda_{3,+}-\lambda_{3,-}\right)}=e^{n\left(\lambda_{4,+}-\lambda_{4,-}\right)}=e^{n\left(-\frac{1}{3} \pi i\right)}=(-1)^{n}
$$

since $n$ is a multiple of three. This explains the entries $(-1)^{n}$ in (6.17) on $\left(-c_{3}, c_{3}\right)$. 


\section{CHAPTER 7}

\section{Opening of Lenses}

The next step in the steepest descent analysis is the opening of lenses around $S\left(\mu_{1}\right), S\left(\sigma_{2}-\mu_{2}\right)$ and $S\left(\mu_{3}\right)$. This will be done in the third and fourth transformations $U \mapsto T$ and $T \mapsto S$.

\subsection{Third transformation $U \mapsto T$}

In the third transformation we open the lenses around $S\left(\sigma_{2}-\mu_{2}\right)$ and $S\left(\mu_{3}\right)$ that will be denoted by $L_{2}$ and $L_{3}$, respectively. The lenses will be closed unbounded sets that do not intersect. There are three situations, depending on whether $c_{2}$ and $c_{3}$ are positive or zero. We recall that by regularity we can not have $c_{2}=c_{3}=0$. The three different cases differ in the shapes of the lens, which is due to the different supports of $S\left(\sigma_{2}-\mu_{2}\right)$ and $S\left(\mu_{3}\right)$ as illustrated in Figures [7.1 7.2 and 7.3.

- In the Cases IV and V we have that both $c_{2}$ and $c_{3}$ are positive and we choose the lips of the lenses such that they have $\pm i c_{2}$ and $\pm c_{3}$ as endpoints, as shown in Figure 7.1

- In Case III we have $c_{2}=0$ and $c_{3}>0$, and now we open the lens such that the lips around $S\left(\sigma_{2}-\mu_{2}\right)=i \mathbb{R}$ stay away from the imaginary axis and intersecting the real line at two points $\pm \gamma_{3}$ as in Figure 7.2. We recall that in Case III we have $0 \notin S\left(\mu_{1}\right)$ and hence the number $N$ of intervals in $S\left(\mu_{1}\right)$ is even. We then choose $\gamma_{3}$ such that

$$
0<\gamma_{3}<\min \left(a_{N / 2+1}, c_{3}\right), \quad \text { in Case III. }
$$

The lens around $S\left(\mu_{3}\right)$ is as in the Cases IV and V.

- In the cases I and II we have $c_{2}>0$ and $c_{3}=0$. We then take the lens around $S\left(\sigma_{2}-\mu_{2}\right)$ as in the Cases IV and V above, but we choose the lips of the lens around $S\left(\mu_{3}\right)=\mathbb{R}$ such that it is away from the real axis and intersects the imaginary axis at two points $\pm i \gamma_{2}$ with

$$
0<\gamma_{2}<c_{2}, \quad \text { in Cases I and II. }
$$

We choose all lenses to be symmetric with respect to both the real and imaginary axes.

Note that we have $\gamma_{3}$ in Case III and $\gamma_{2}$ in Cases I and II. For ease of presentation we also define

$$
\begin{array}{ll}
\gamma_{2}=0 & \text { in Cases III, IV, and V, } \\
\gamma_{3}=0 & \text { in Cases I, II, IV, and V. }
\end{array}
$$

There are further requirements on the lenses that are important for the steepest descent analysis. These are formulated in the next two lemmas.

LEMma 7.1. We can and do choose the lens $L_{2}$ around $S\left(\sigma_{2}-\mu_{2}\right)$ such that

$$
\operatorname{Re}\left(\lambda_{3}-\lambda_{2}\right)<0 \quad \text { in } L_{2} \backslash i \mathbb{R}
$$




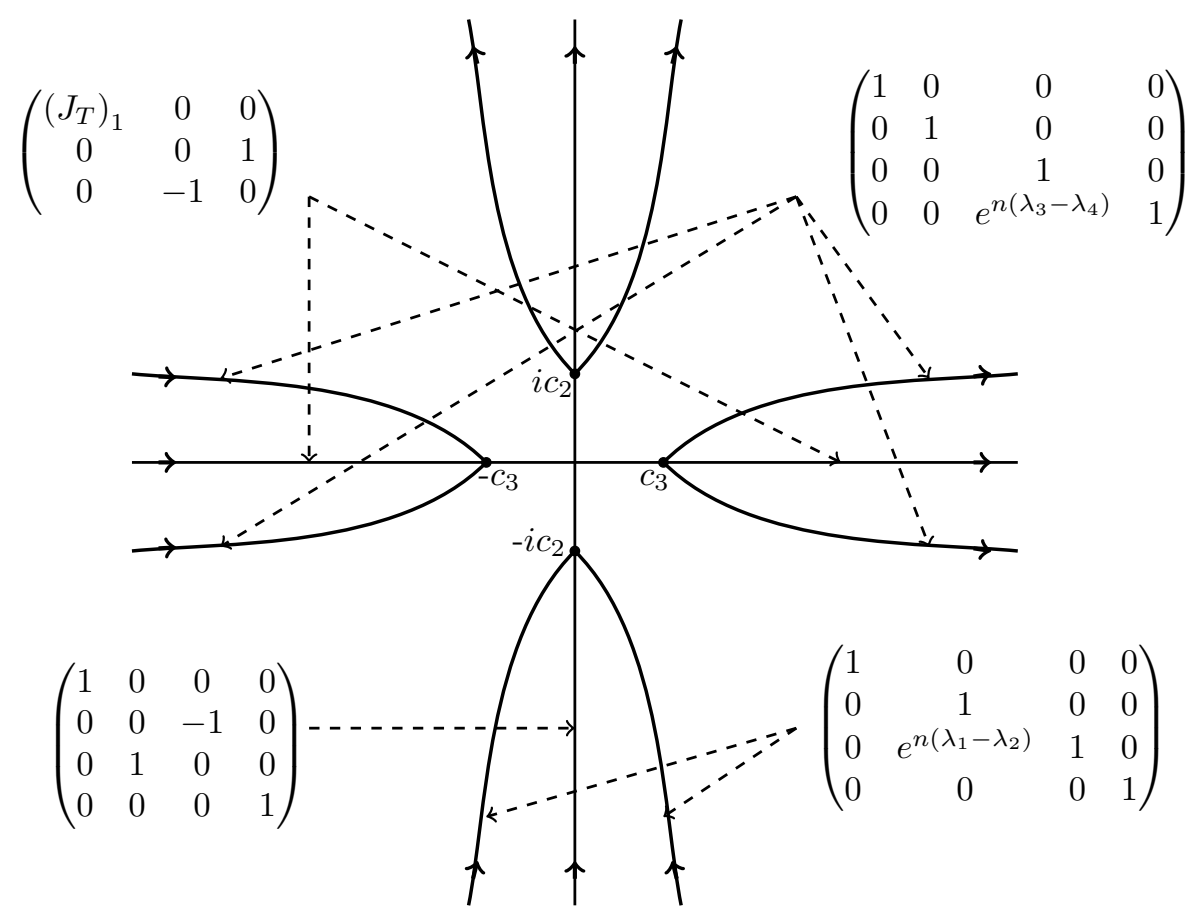

Figure 7.1. Unbounded lenses in case $c_{2}>0$ and $c_{3}>0$, corresponding to Cases IV and V. The figure also shows the jump contour $\Sigma_{T}$ and some of the jump matrices in the $\mathrm{RH}$ problem (7.9) for $T$.

and such that

$$
\{z \in \mathbb{C}|| z|>R,| \operatorname{Re}(z)|<\varepsilon| \operatorname{Im}(z) \mid\} \subset L_{2}
$$

for some $\varepsilon>0$ and $R>0$.

Proof. From (4.35) we obtain

$$
\lambda_{3}-\lambda_{2}=2 g_{2}-g_{1}-g_{3}+\theta_{2}-\theta_{1}
$$

and for $z \in S\left(\sigma_{2}-\mu_{2}\right)$ we have by (4.11) and (2.10) that

$$
\left(\lambda_{3}-\lambda_{2}\right)_{ \pm}(z)= \pm\left(g_{2,+}(z)-g_{2,-}(z)+\theta_{1,-}(z)-\theta_{1,+}(z)\right) .
$$

Using (4.9), (2.7) and (2.9) we can further rewrite this to

$$
\left(\lambda_{3}-\lambda_{2}\right)_{ \pm}(z)= \begin{cases} \pm \frac{2}{3} \pi i \pm 2 \pi i\left(\sigma_{2}-\mu_{2}\right)([0, z]) & \text { for } \operatorname{Im} z>0 \\ \mp \frac{2}{3} \pi i \mp 2 \pi i\left(\sigma_{2}-\mu_{2}\right)([z, 0]) & \text { for } \operatorname{Im} z<0\end{cases}
$$

which is purely imaginary. Then

$$
\frac{d}{d y} \operatorname{Im}\left( \pm\left(\lambda_{3}-\lambda_{2}\right)_{ \pm}(i y)\right)=2 \pi \frac{d\left(\sigma_{2}-\mu_{2}\right)}{|d z|}(i y)
$$

which is positive for $y \in \mathbb{R}$ in Case III, and for $|y|>c_{2}>0$ in the other cases.

The first statement of the lemma then follows from the Cauchy Riemann equations since $\operatorname{Re}\left(\lambda_{3}-\lambda_{2}\right)_{ \pm}=0$ on the imaginary axis. The second statement follows 


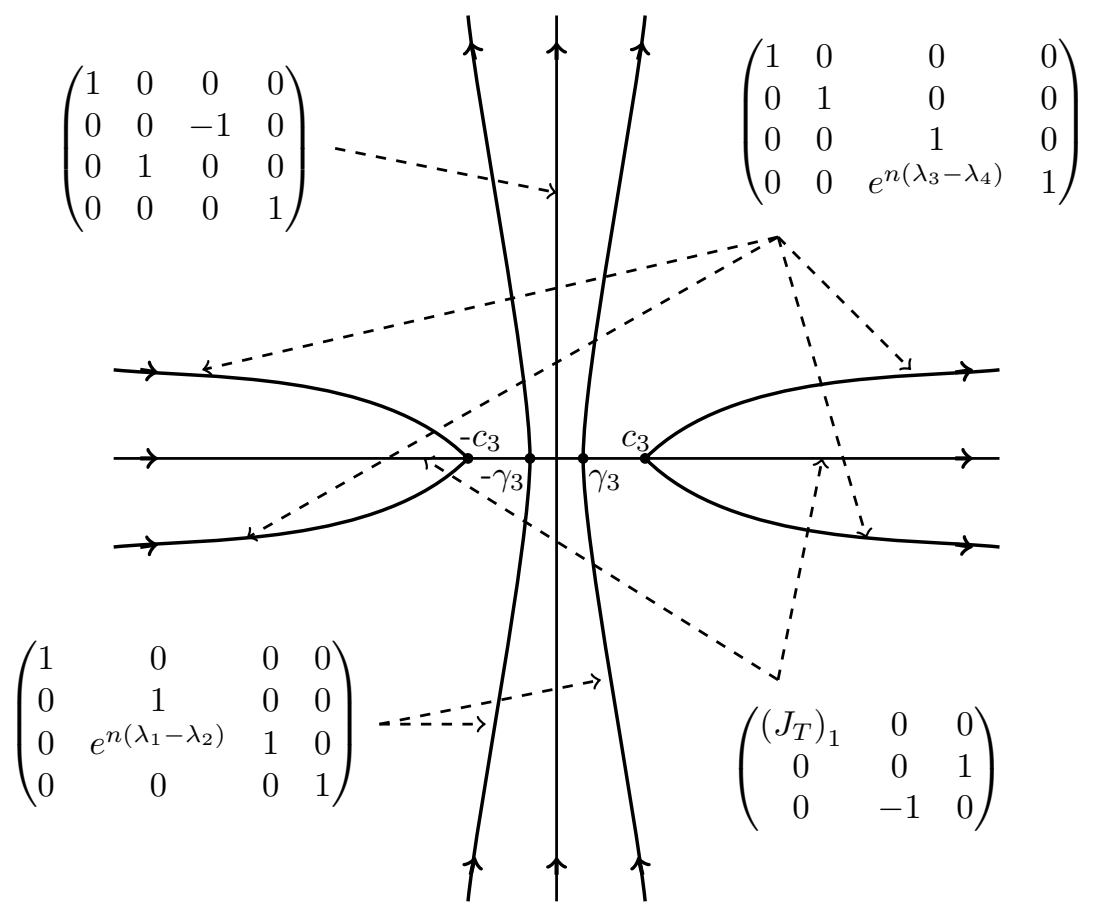

FiguRE 7.2. Unbounded lenses in case $c_{2}=0$ and $c_{3}>0$, corresponding to Case III. The figure also shows the jump contour $\Sigma_{T}$ and some of the jump matrices in the RH problem (7.9) for $T$.

then from the asymptotic behavior of $\lambda_{2}$ and $\lambda_{3}$ as given in (4.47), (4.48) and Lemma 2.4 .

Lemma 7.2. We can and do choose the lens $L_{3}$ around $S\left(\mu_{3}\right)$ such that

$$
\operatorname{Re}\left(\lambda_{3}-\lambda_{4}\right)<0 \quad \text { in } L_{3} \backslash \mathbb{R}
$$

and such that

$$
\{z \in \mathbb{C}|| z|>R,| \operatorname{Im}(z)|<\varepsilon| \operatorname{Re}(z) \mid\} \subset L_{3}
$$

for some $\varepsilon>0$ and $R>0$.

Proof. The proof is similar to the proof of Lemma 7.1 .

Using (4.35) we obtain

$$
\lambda_{3}-\lambda_{4}=-2 g_{3}+g_{2}+\theta_{2}-\theta_{3} .
$$

and hence

$$
\lambda_{3, \pm}-\lambda_{4, \pm}=-g_{3,+}-g_{3,-}+g_{2, \pm}+\theta_{2, \pm}-\theta_{3, \pm} \pm\left(g_{3,-}-g_{3,+}\right) .
$$

Now by (2.15) and (5.33)

$$
\theta_{2, \pm}(x)-\theta_{3, \pm}(x)= \begin{cases}V_{3}(x) \pm 2 \pi i \sigma_{3}\left(\left[x^{*}(\alpha), x\right]\right), & x>0 \\ V_{3}(x) \mp 2 \pi i \sigma_{3}\left(\left[x,-x^{*}(\alpha)\right]\right), & x<0\end{cases}
$$




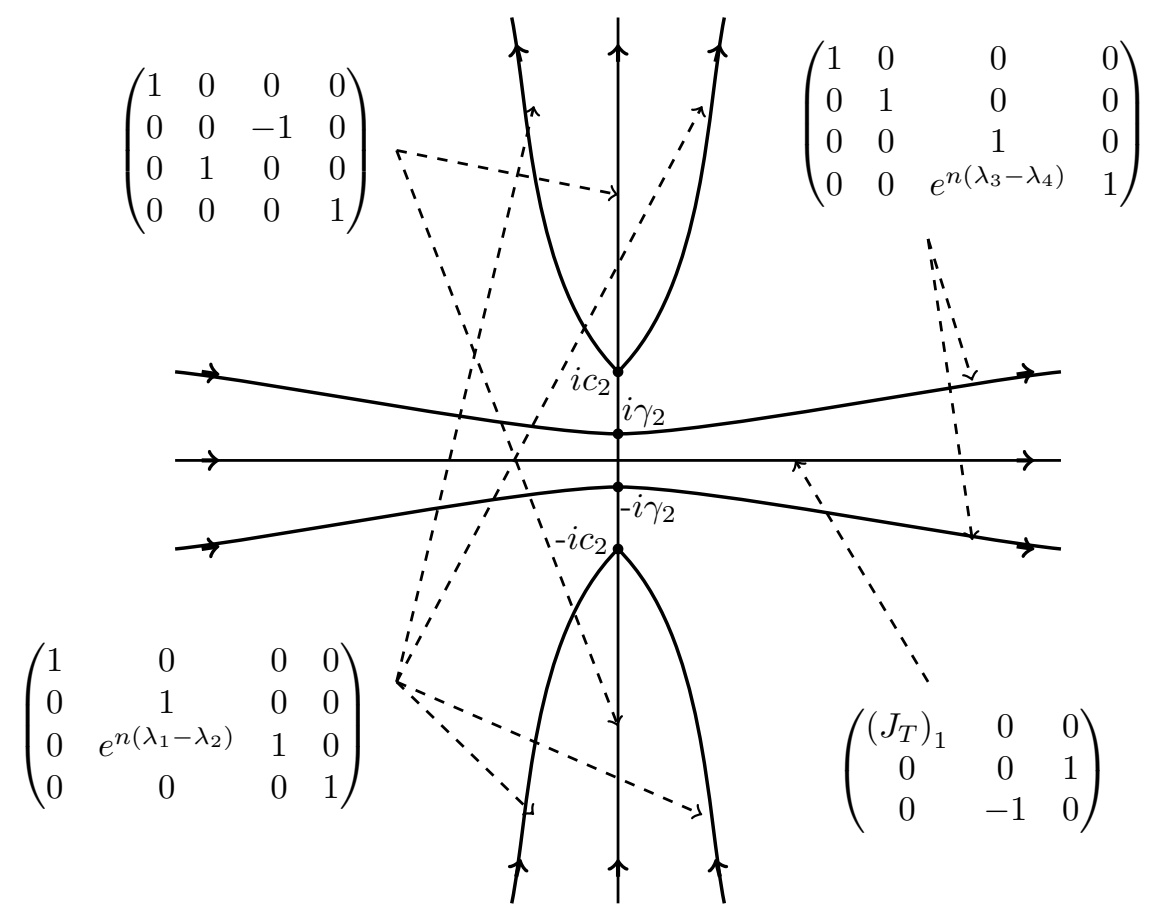

Figure 7.3. Unbounded lenses in case $c_{2}>0$ and $c_{3}=0$, corresponding to Cases I and II. The figure also shows the jump contour $\Sigma_{T}$ and some of the jump matrices in the RH problem (7.9) for $T$.

Combining this with (4.12) and (4.7) leads to

$$
\lambda_{3, \pm}(x)-\lambda_{4, \pm}(x)= \pm 2 \pi i \times \begin{cases}\sigma_{3}\left(\left[x^{*}(\alpha), x\right]\right)-\mu_{3}([x, \infty)), & x>c_{3}, \\ -\sigma_{3}\left(\left[x,-x^{*}(\alpha)\right)-\mu_{3}([x, \infty))-\frac{1}{3},\right. & x<-c_{3},\end{cases}
$$

which is purely imaginary. We find

$$
\frac{d}{d x} \operatorname{Im}\left( \pm\left(\lambda_{3}-\lambda_{4}\right)_{ \pm}(x)\right)=2 \pi \frac{d\left(\sigma_{3}+\mu_{3}\right)}{d x}(x)
$$

and this is positive for $x \in \mathbb{R}$ in Cases I and II, and for $|x|>c_{3}>0$ in the other cases.

As in Lemma 7.1 the first statement of the lemma now follows by the Cauchy Riemann equations. The second statement follows by (4.48), (4.49), and Lemma 2.4.

Now that we have defined the lenses $L_{2}$ and $L_{3}$ we can come to the actual definition of the transformation $U \mapsto T$. The transformation is based on the following factorization of $\left(J_{U}\right)_{2}$ on $S\left(\sigma_{2}-\mu_{2}\right)$ as given by (6.16) (recall that $\lambda_{2, \pm}=\lambda_{3, \mp}$ )

$$
\begin{aligned}
\left(J_{U}\right)_{2} & =\left(\begin{array}{cc}
e^{n\left(\lambda_{3}-\lambda_{2}\right)_{-}} & 0 \\
1 & e^{n\left(\lambda_{3}-\lambda_{2}\right)_{+}}
\end{array}\right) \\
& =\left(\begin{array}{cc}
1 & e^{n\left(\lambda_{3}-\lambda_{2}\right)_{-}} \\
0 & 1
\end{array}\right)\left(\begin{array}{cc}
0 & -1 \\
1 & 0
\end{array}\right)\left(\begin{array}{cc}
1 & e^{n\left(\lambda_{3}-\lambda_{2}\right)_{+}} \\
0 & 1
\end{array}\right)
\end{aligned}
$$


and the factorization of $\left(J_{U}\right)_{3}$ on $S\left(\mu_{3}\right)$ as given by (6.17) (recall that $\lambda_{3, \pm}=\lambda_{4, \mp}$ or $\lambda_{3, \pm}=\lambda_{4, \mp} \pm \frac{2}{3} \pi i$ and $n$ is a multiple of three)

$$
\begin{aligned}
\left(J_{U}\right)_{3} & =\left(\begin{array}{cc}
e^{n\left(\lambda_{3}-\lambda_{4}\right)_{+}} & 1 \\
0 & e^{n\left(\lambda_{3}-\lambda_{4}\right)_{-}}
\end{array}\right) \\
& =\left(\begin{array}{cc}
1 & 0 \\
e^{n\left(\lambda_{3}-\lambda_{4}\right)_{-}} & 1
\end{array}\right)\left(\begin{array}{cc}
0 & 1 \\
-1 & 0
\end{array}\right)\left(\begin{array}{cc}
1 & 0 \\
e^{n\left(\lambda_{3}-\lambda_{4}\right)_{+}} & 1
\end{array}\right) .
\end{aligned}
$$

This leads to the following definition of $T$.

Definition 7.3. We define the $4 \times 4$ matrix valued function $T$ by

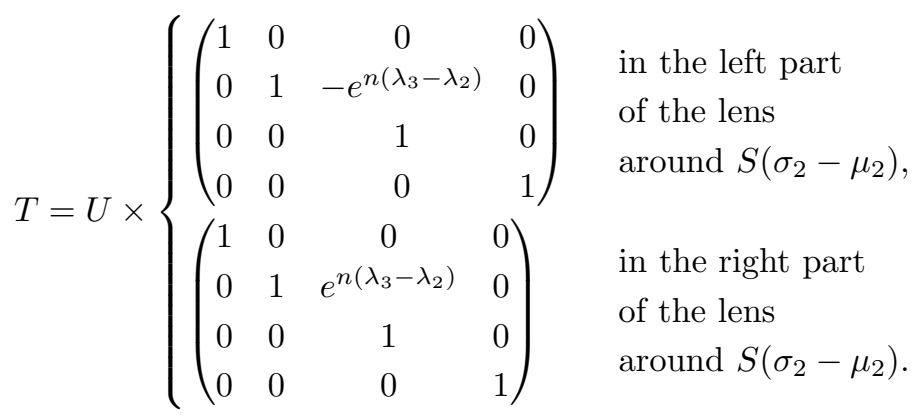

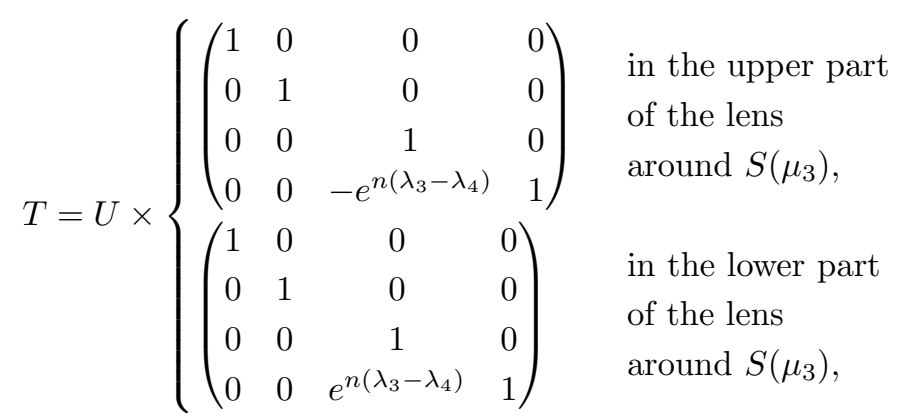

and

$$
T=U \text { elsewhere. }
$$

Then $T$ is defined and analytic in $\mathbb{C} \backslash \Sigma_{T}$ where $\Sigma_{T}$ is the contour consisting of the real and imaginary axes and the lips of the lenses around $S\left(\sigma_{2}-\mu_{2}\right)$ and $S\left(\mu_{3}\right)$.

\subsection{RH problem for $T$}

Now $T$ solves the following RH problem

$$
\left\{\begin{array}{l}
T \text { is analytic in } \mathbb{C} \backslash \Sigma_{T}, \\
T_{+}=T_{-} J_{T}, \quad \text { on } \Sigma_{T}, \\
T(z)=\left(I+\mathcal{O}\left(z^{-1 / 3}\right)\right) \operatorname{diag}\left(\begin{array}{llll}
1 & z^{\frac{1}{3}} & 1 & z^{-\frac{1}{3}}
\end{array}\right)\left(\begin{array}{cc}
1 & 0 \\
0 & A_{j}
\end{array}\right) \\
\text { as } z \rightarrow \infty \text { in the } j \text { th quadrant, }
\end{array}\right.
$$

with certain jump matrices $J_{T}$ that will be described in the next section. The matrices $A_{j}$ are given in (5.13)-(5.14). The contour $\Sigma_{T}$ and some of the jump matrices $J_{T}$ are shown in Figures [7.1, 7.2 and 7.3. 
Since the lenses around $S\left(\sigma_{2}-\mu_{2}\right)$ and $S\left(\mu_{3}\right)$ are unbounded, we have to be careful about the asymptotic behavior of $T(z)$ as $z \rightarrow \infty$, since it could be different from the asymptotic behavior of $U(z)$. However, a simple check using (4.47)-(4.49), (7.6) and (7.7) shows that the asymptotic behavior is the same.

However, it is good to note the following. The asymptotic behavior of the Pearcey functions given in (5.9) is not uniform up to the real and imaginary axes. By following the transformations $Y \mapsto X \mapsto U$ we see that the same is true for the asymptotic behavior of $U$. It requires an independent check that after opening of the unbounded lenses the asymptotic behavior of $T$ is in fact uniform in each of the quadrants. This phenomenon also appeared in 45 .

\subsection{Jump matrices for $T$}

Our next task is to compute the jump matrices $J_{T}$ in the Riemann-Hilbert problem (7.9) for $T$. The definitions (7.6) and (7.7) of $T$ and the structure of the jump matrix $J_{U}$ as given in (6.7) and (6.8) yield that

$$
T_{+}=T_{-} J_{T}
$$

where $J_{T}$ has again the structure

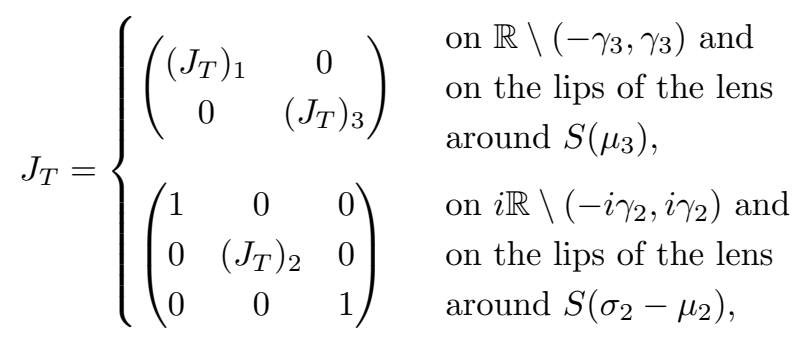

with $2 \times 2$ blocks $\left(J_{T}\right)_{k}$ for $k=1,2,3$. The block structure as in (7.10) is not valid on the intervals $\left(-\gamma_{3}, \gamma_{3}\right)$ and $\left(-i \gamma_{2}, i \gamma_{2}\right)$ (if non-empty). On these intervals the block structure changes to

$$
\begin{array}{rlr}
J_{T}=\left(\begin{array}{cc}
\left(J_{T}\right)_{1} & * \\
0 & \left(J_{T}\right)_{3}
\end{array}\right) & \text { on }\left(-\gamma_{3}, \gamma_{3}\right), \\
J_{T}=\left(\begin{array}{ccc}
1 & 0 & 0 \\
0 & \left(J_{T}\right)_{2} & 0 \\
0 & * & 1
\end{array}\right) & \text { on }\left(-i \gamma_{2}, i \gamma_{2}\right),
\end{array}
$$

with some non-zero entries that are denoted by $*$, see (7.15) and (7.16) below.

The diagonal blocks are given in the next lemma.

Lemma 7.4. (a) For $\left(J_{T}\right)_{1}$ we have

$$
\left(J_{T}\right)_{1}= \begin{cases}\left(J_{U}\right)_{1} & \text { on } \mathbb{R}, \\ I_{2} & \text { on the lips of the lens around } S\left(\mu_{3}\right),\end{cases}
$$

where $\left(J_{U}\right)_{1}$ is given by (6.15). 
(b) For $\left(J_{T}\right)_{2}$ we have

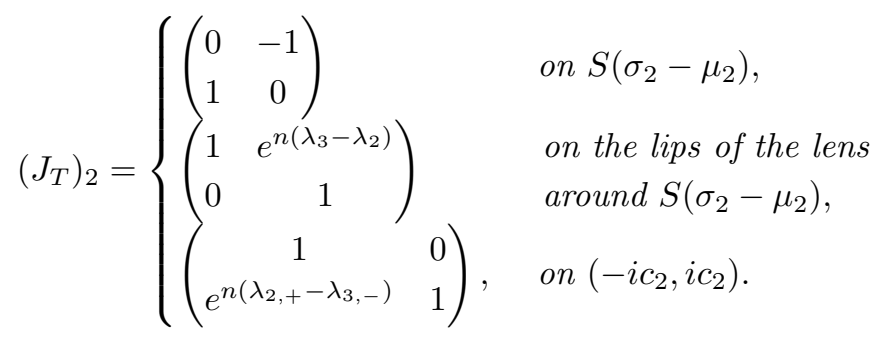

(c) For $\left(J_{T}\right)_{3}$ we have

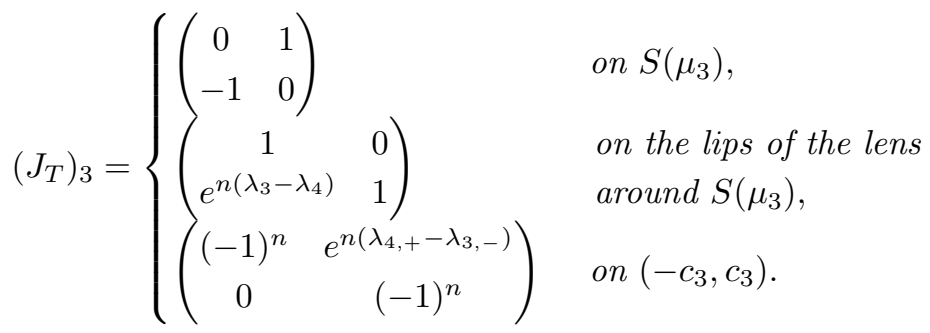

Proof. The jumps follow from the jumps $J_{U}$ for $U$ in 6.15) 6.17) and the transformation (7.6)-(7.8), where we use the factorizations (7.4) and (7.5).

Outside the lenses the blocks $\left(J_{T}\right)_{2}$ and $\left(J_{T}\right)_{3}$ have not changed, and so on $\left(-i c_{2}, i c_{2}\right)=i \mathbb{R} \backslash S\left(\sigma_{2}-\mu_{2}\right)$ we have $\left(J_{T}\right)_{2}=\left(J_{U}\right)_{2}$ and on $\left(-c_{3}, c_{3}\right)=\mathbb{R} \backslash S\left(\mu_{3}\right)$ we have $\left(J_{T}\right)_{3}=\left(J_{U}\right)_{3}$. See also (7.16) and (7.15) for the result of the calculation of the full jump matrix $J_{T}$ on the intervals $\left(-i \gamma_{2}, i \gamma_{2}\right)$ and $\left(-\gamma_{3}, \gamma_{3}\right)$.

It remains to describe the off-diagonal entries in (17.11) that only occur in case $\gamma_{3}>0$ or $\gamma_{2}>0$, that is in Cases III or I/II.

Case III: $\gamma_{3}>0$. In Case III we have $c_{2}=0$ and the lips of the lens around $S\left(\sigma_{2}-\mu_{2}\right)$ intersect the real line at $\pm \gamma_{3}$ with $\gamma_{3}>0$.

In order to compute the jump matrix $J_{T}$ on $\left(-\gamma_{3}, \gamma_{3}\right)$, we have to note that by the regularity assumption we have that $0 \notin S\left(\mu_{1}\right) \cup S\left(\mu_{3}\right)$, and by the choice of $\gamma_{3}$ in (7.1) we have that $\left[-\gamma_{3}, \gamma_{3}\right]$ is disjoint from $S\left(\mu_{1}\right) \cap S\left(\mu_{3}\right)$. Also, the measure $\mu_{1}$ is symmetric so that $\alpha_{N / 2}=1 / 2$. Hence the jump matrix $J_{U}$ given in (6.7), (6.15) and (6.17) takes the form

$$
J_{U}=\left(\begin{array}{cccc}
(-1)^{n} & e^{n\left(\lambda_{2,+}-\lambda_{1,-}\right)} & 0 & 0 \\
0 & (-1)^{n} & 0 & 0 \\
0 & 0 & (-1)^{n} & e^{n\left(\lambda_{4,+}-\lambda_{3,-}\right)} \\
0 & 0 & 0 & (-1)^{n}
\end{array}\right) \quad \text { on }\left(-\gamma_{3}, \gamma_{3}\right)
$$

From (7.6) we obtain

$$
J_{T}=\left(\begin{array}{cccc}
1 & 0 & 0 & 0 \\
0 & 1 & \mp e^{n\left(\lambda_{3}-\lambda_{2}\right)} & 0 \\
0 & 0 & 1 & 0 \\
0 & 0 & 0 & 1
\end{array}\right) J_{U}\left(\begin{array}{cccc}
1 & 0 & 0 & 0 \\
0 & 1 & \pm e^{n\left(\lambda_{3}-\lambda_{2}\right)} & 0 \\
0 & 0 & 1 & 0 \\
0 & 0 & 0 & 1
\end{array}\right)
$$

on $\left(-\gamma_{3}, \gamma_{3}\right) \cap \mathbb{R}^{ \pm}$. 
After some calculations using (4.37), (4.38), (4.42) and the fact that $n$ is a multiple of 3 we obtain from the last two expressions that

$$
J_{T}=\left(\begin{array}{cccc}
(-1)^{n} & e^{n\left(\lambda_{2,+}-\lambda_{1,-}\right)} & \pm e^{n\left(\lambda_{3,+}-\lambda_{1,-}\right)} & 0 \\
0 & (-1)^{n} & 0 & \mp e^{n\left(\lambda_{4,+}-\lambda_{2,-}\right)} \\
0 & 0 & (-1)^{n} & e^{n\left(\lambda_{4,+}-\lambda_{3,-}\right)} \\
0 & 0 & 0 & (-1)^{n}
\end{array}\right)
$$

Cases I and II: $\gamma_{2}>0$. In Cases I and II we have $c_{3}=0$ and the lips of the lens around $S\left(\mu_{3}\right)$ intersect the imaginary axis at $\pm i \gamma_{2}$ with $\gamma_{2}>0$. By (6.8) and (6.16) we have

$$
J_{U}=\left(\begin{array}{cccc}
1 & 0 & 0 & 0 \\
0 & 1 & 0 & 0 \\
0 & e^{n\left(\lambda_{2,+}-\lambda_{3,-}\right)} & 1 & 0 \\
0 & 0 & 0 & 1
\end{array}\right) \quad \text { on }\left(-i \gamma_{2}, i \gamma_{2}\right)
$$

Combining this with (7.7) and using (4.40), the fact that $\lambda_{4}$ is analytic on $i \mathbb{R} \backslash\{0\}$ and the fact that $n$ is a multiple of 3 leads to

$$
J_{T}=\left(\begin{array}{cccc}
1 & 0 & 0 & 0 \\
0 & 1 & 0 & 0 \\
0 & e^{n\left(\lambda_{2,+}-\lambda_{3,-}\right)} & 1 & 0 \\
0 & \pm e^{n\left(\lambda_{2,+}-\lambda_{4,-}\right)} & 0 & 1
\end{array}\right) \quad \text { on }\left(-i \gamma_{2}, i \gamma_{2}\right) \cap i \mathbb{R}^{ \pm}
$$

\subsection{Fourth transformation $T \mapsto S$}

In the fourth transformation we open up a lens around each interval $\left[a_{k}, b_{k}\right]$ of $S\left(\mu_{1}\right)$. The union of these lenses will be denoted by $L_{1}$ and $L_{1}$ is a closed bounded set. This is done in a standard way based on the factorization

$$
\begin{aligned}
\left(J_{T}\right)_{1}=\left(J_{U}\right)_{1} & =\left(\begin{array}{cc}
e^{n\left(\lambda_{1}-\lambda_{2}\right)_{+}} & 1 \\
0 & e^{n\left(\lambda_{1}-\lambda_{2}\right)_{-}}
\end{array}\right) \\
& =\left(\begin{array}{cc}
1 & 0 \\
e^{n\left(\lambda_{1}-\lambda_{2}\right)_{-}} & 1
\end{array}\right)\left(\begin{array}{cc}
0 & 1 \\
-1 & 0
\end{array}\right)\left(\begin{array}{cc}
1 & 0 \\
e^{n\left(\lambda_{1}-\lambda_{2}\right)_{+}} & 1
\end{array}\right) .
\end{aligned}
$$

LEMMA 7.5. We can and do choose the lenses $L_{1}$ around $S\left(\mu_{1}\right)$ such that

$$
\operatorname{Re}\left(\lambda_{1}-\lambda_{2}\right)<0 \quad \text { in } L_{1} \backslash \mathbb{R} \text {. }
$$

Proof. By (4.35) we obtain

$$
\left(\lambda_{1}-\lambda_{2}\right)_{ \pm}=V-l_{1}-2 g_{1, \pm}+g_{2, \pm}-\theta_{1} .
$$

By (4.10), (2.14) and (4.3) this leads to

$$
\left(\lambda_{1}-\lambda_{2}\right)_{ \pm}(x)= \begin{cases}\mp\left(g_{1,+}(x)-g_{1,-}(x)\right)= \pm 2 \pi i \mu_{1}([x, \infty)), & x>0, \\ \mp\left(g_{1,+}(x)-g_{1,-}(x)\right) \pm \frac{2}{3} \pi i= \pm 2 \pi i \mu_{1}([x, \infty)) \pm \frac{2}{3} \pi i, & x<0 .\end{cases}
$$

Thus $\pm\left(\lambda_{1}-\lambda_{2}\right)_{ \pm}$is purely imaginary and

$$
\frac{d}{d x} \operatorname{Im}\left( \pm\left(\lambda_{1}-\lambda_{2}\right)_{ \pm}(x)\right)=2 \pi \frac{d \mu_{1}}{d x}
$$




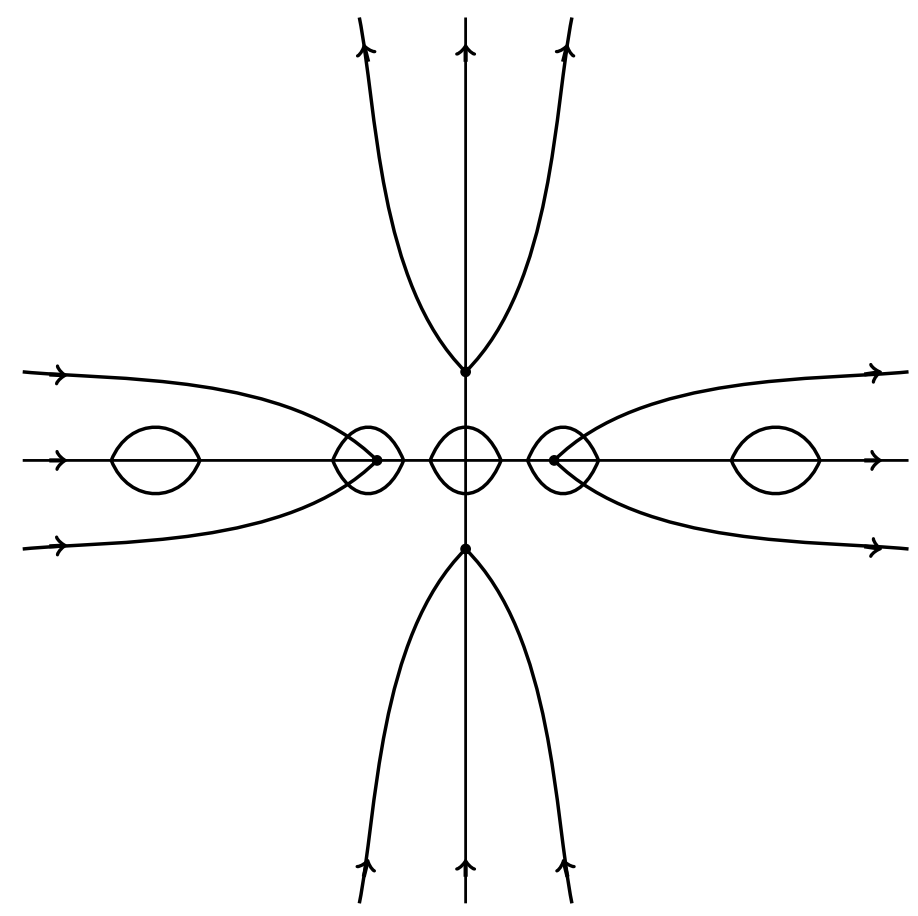

Figure 7.4. The opening of the lenses around $S\left(\mu_{1}\right)$. The lens around $\left(a_{k}, b_{k}\right)$ intersects the lens around $S\left(\mu_{3}\right)$ if and only if $\pm c_{3} \in$ $\left(a_{k}, b_{k}\right)$ as is the case in the figure.

which is strictly positive on each of the intervals $\left(a_{k}, b_{k}\right)$ by the regularity assumption on $\mu_{1}$. The lemma follows by the Cauchy-Riemann equations.

In addition to the condition described in the lemma we also make sure that the lenses around $S\left(\mu_{1}\right)$ do not intersect the lens around $S\left(\sigma_{2}-\mu_{2}\right)$ and the lips of the lens around $S\left(\mu_{3}\right)$, except for the case when $\pm c_{3} \in S\left(\mu_{1}\right)$. In that case we choose the lips around the interval(s) containing $\pm c_{3}$ in such a way that they intersect the lips of the lens around $S\left(\mu_{3}\right)$ exactly once in each quadrant as it is shown in Figure 7.4

If $0 \in S\left(\mu_{1}\right)$ then the lips of the lens around the interval containing 0 intersect the imaginary axis in the two points $\pm i \widehat{\gamma}_{2}$ where

$$
0<\widehat{\gamma}_{2}<c_{2} \text {. }
$$

See also Figure 7.4, If in addition $0 \in S\left(\mu_{3}\right)$ then also

$$
0<\widehat{\gamma}_{2}<\gamma_{2}
$$

If $0 \notin S\left(\mu_{1}\right)$ then we put

$$
\widehat{\gamma}_{2}=0 \text {. }
$$

We also take the lenses such that they are symmetric in both the real and imaginary axis. We now define $S$ as follows. 
Definition 7.6. We define

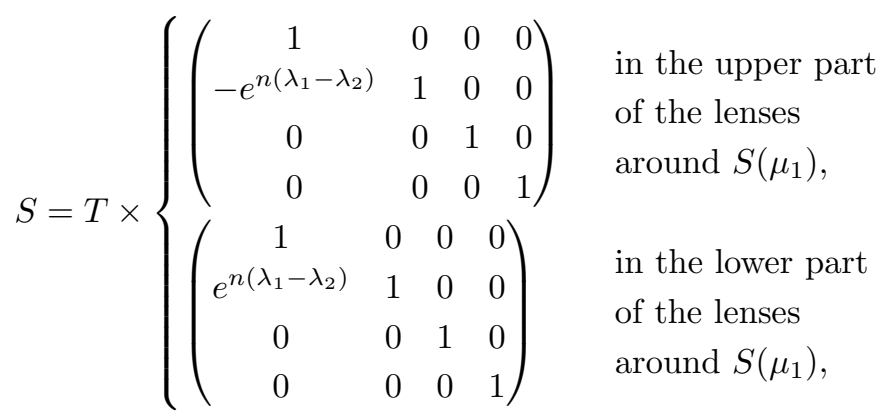

and

$$
S=T \quad \text { elsewhere. }
$$

Then $S$ is defined and analytic in $\mathbb{C} \backslash \Sigma_{S}$ where $\Sigma_{S}$ is the contour consisting of the real and imaginary axis and the lips of the lenses around $S\left(\mu_{1}\right), S\left(\sigma_{2}-\mu_{2}\right)$ and $S\left(\mu_{3}\right)$.

\subsection{RH problem for $S$}

The asymptotic behavior as $z \rightarrow \infty$ clearly has not changed, and so $S$ satisfies the following $\mathrm{RH}$ problem.

$$
\left\{\begin{array}{l}
S \text { is analytic in } \mathbb{C} \backslash \Sigma_{S}, \\
S_{+}=S_{-} J_{S}, \quad \text { on } \Sigma_{S}, \\
S(z)=\left(I+\mathcal{O}\left(z^{-1 / 3}\right)\right) \operatorname{diag}\left(\begin{array}{llll}
1 & z^{\frac{1}{3}} & 1 & z^{-\frac{1}{3}}
\end{array}\right)\left(\begin{array}{cc}
1 & 0 \\
0 & A_{j}
\end{array}\right) \\
\text { as } z \rightarrow \infty \text { in the } j \text { th quadrant }
\end{array}\right.
$$

with jump matrices $J_{S}$ that are described next.

The jump matrices $J_{S}$ have again the block structure

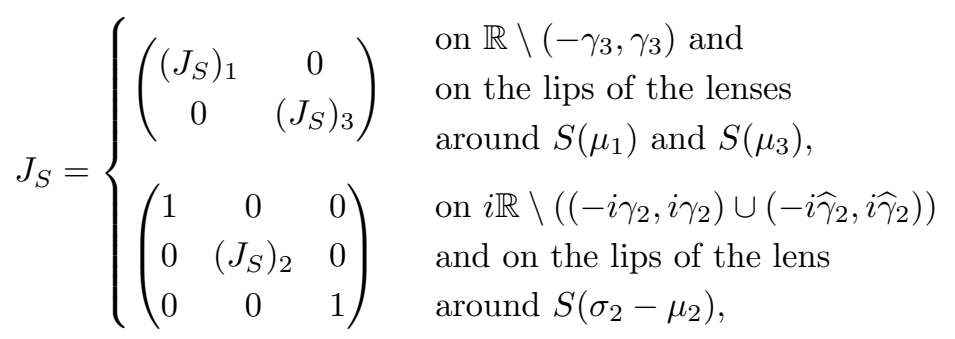

with $2 \times 2$ blocks $\left(J_{S}\right)_{k}$ for $k=1,2,3$. The block structure is different on the intervals $\left(-\gamma_{3}, \gamma_{3}\right),\left(-i \gamma_{2}, i \gamma_{2}\right)$ and $\left(-i \widehat{\gamma}_{2}, i \widehat{\gamma}_{2}\right)$ if non-empty.

On these intervals we have

$$
\begin{aligned}
& J_{S}=\left(\begin{array}{cc}
\left(J_{S}\right)_{1} & * \\
0 & \left(J_{S}\right)_{3}
\end{array}\right) \quad \text { on }\left(-\gamma_{3}, \gamma_{3}\right) \\
& J_{S}=\left(\begin{array}{ccc}
1 & 0 & 0 \\
* & \left(J_{S}\right)_{2} & 0 \\
* & * & 1
\end{array}\right) \quad \text { on }\left(-i \gamma_{2}, i \gamma_{2}\right) \text { or }\left(-i \widehat{\gamma}_{2}, i \widehat{\gamma}_{2}\right),
\end{aligned}
$$

with possible non-zero entries that are denoted by $*$. 
The diagonal blocks are given in the following lemma.

LEMMA 7.7. (a) For $\left(J_{S}\right)_{1}$ we have

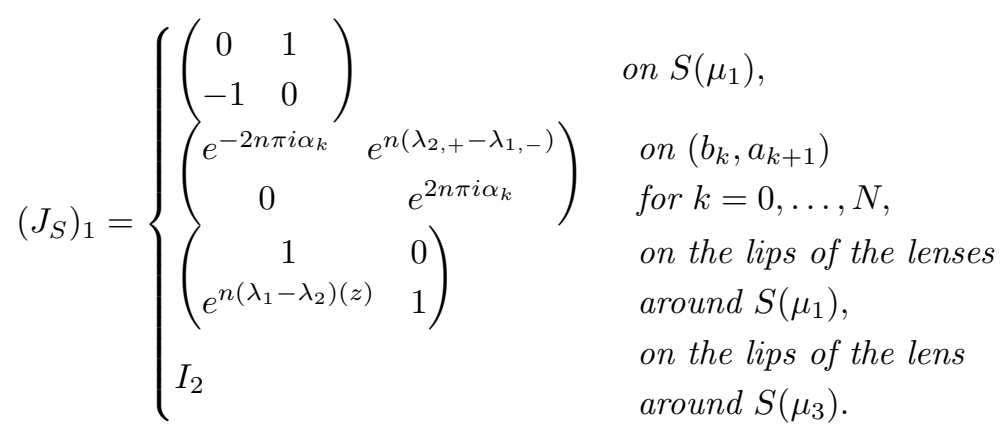

(b) For $\left(J_{S}\right)_{2}$ we have

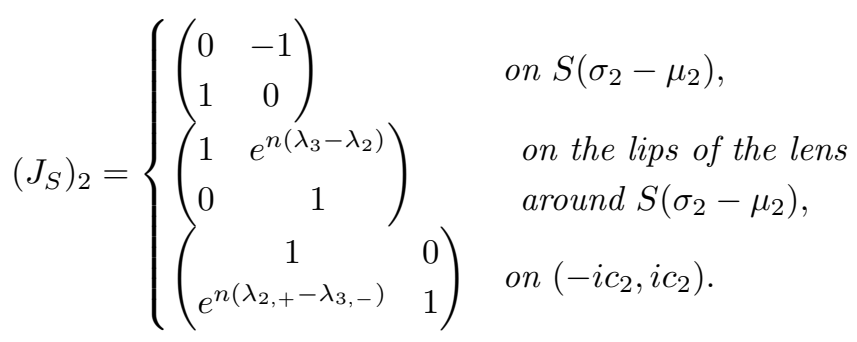

(c) For $\left(J_{S}\right)_{3}$ we have

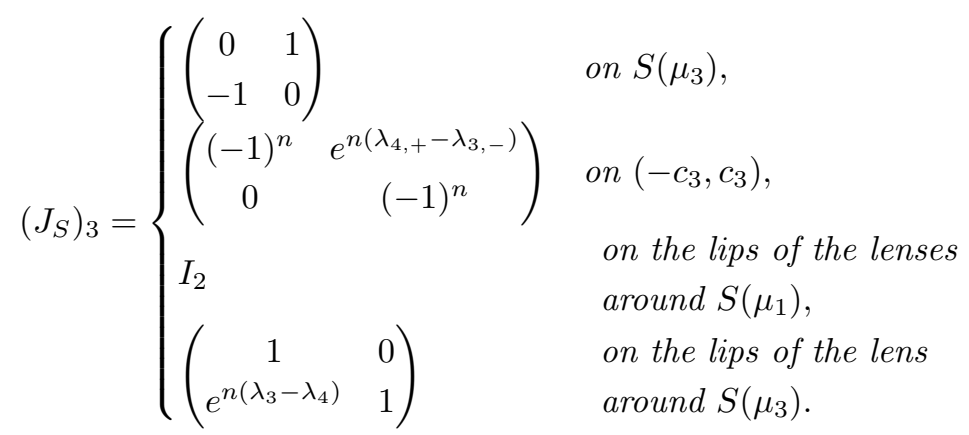

Proof. The expressions for $\left(J_{S}\right)_{1}$ on $S\left(\mu_{1}\right)$ and on the lips of the lenses around $S\left(\mu_{1}\right)$ follow from the factorization (7.17) and the transformation (7.18)-(7.19). On the other contours we have $\left(J_{S}\right)_{1}=\left(J_{T}\right)_{1}$. Also $\left(J_{S}\right)_{k}=\left(J_{T}\right)_{k}$ for $k=2,3$, and so we find all further expressions in the lemma from (7.12)-(7.14).

We next give the jump matrices $J_{S}$ on the intervals $\left(-\gamma_{3}, \gamma_{3}\right),\left(-i \gamma_{2}, i \gamma_{2}\right)$ and $\left(-i \widehat{\gamma}_{2}, i \widehat{\gamma}_{2}\right)$ and in particular the off-diagonal entries that were denoted by $*$ in (17.22). They depend on the Cases I-V. We simply present the formulas without further comment. Of course they follow from the jump matrices $J_{T}$ and the tranformation (7.18)-(7.19). Note that

$$
\widehat{\gamma}_{2}>0 \Leftrightarrow 0 \in S\left(\mu_{1}\right), \quad \gamma_{3}>0 \Leftrightarrow c_{2}=0, \quad \gamma_{2}>0 \Leftrightarrow c_{3}=0
$$


Case I. In Case I we have $0 \in S\left(\mu_{1}\right), c_{2}>0$ and $c_{3}=0$ so that $\widehat{\gamma}_{2}>0$ and $\gamma_{2}>0$ and $\gamma_{3}=0$. By construction $\widehat{\gamma}_{2}<\gamma_{2}$ and we find in the Case I

$$
J_{S}=\left\{\begin{array}{cccc}
\left(\begin{array}{cccc}
1 & 0 & 0 & 0 \\
0 & 1 & 0 & 0 \\
\mp e^{n\left(\lambda_{1}-\lambda_{3,-}\right)} & e^{n\left(\lambda_{2,+}-\lambda_{3,-}\right)} & 1 & 0 \\
-e^{n\left(\lambda_{1}-\lambda_{4}\right)} & \pm e^{n\left(\lambda_{2,+}-\lambda_{4}\right)} & 0 & 1
\end{array}\right) \quad \text { on }\left(-i \widehat{\gamma}_{2}, i \widehat{\gamma}_{2}\right) \cap i \mathbb{R}^{ \pm} \\
J_{T} & & & \text { on }\left(-i \gamma_{2},-i \widehat{\gamma}_{2}\right) \cup\left(i \widehat{\gamma}_{2}, i \gamma_{2}\right),
\end{array}\right.
$$

where $J_{T}$ is given by (7.16).

Case II. In Case II we have $0 \notin S\left(\mu_{1}\right), c_{2}>0$ and $c_{3}=0$ so that $\widehat{\gamma}_{2}=0$, $\gamma_{2}>0$ and $\gamma_{3}=0$. In this case

$$
J_{S}=J_{T} \quad \text { on }\left(-i \gamma_{2}, i \gamma_{2}\right)
$$

where $J_{T}$ is given by (7.16).

Case III. In Case III we have $0 \notin S\left(\mu_{1}\right), c_{2}=0$ and $c_{3}>0$ so that $\widehat{\gamma}_{2}=0$, $\gamma_{2}=0$ and $\gamma_{3}>0$. We have in this case

$$
J_{S}=J_{T} \quad \text { on }\left(-\gamma_{3}, \gamma_{3}\right)
$$

where $J_{T}$ is given by (7.15).

Case IV. In Case IV we have $0 \in S\left(\mu_{1}\right), c_{2}>0$ and $c_{3}>0$ so that $\widehat{\gamma}_{2}>0$, $\gamma_{2}=0$ and $\gamma_{3}=0$. We have in this case

$$
J_{S}=\left(\begin{array}{cccc}
1 & 0 & 0 & 0 \\
0 & 1 & 0 & 0 \\
\mp e^{n\left(\lambda_{1}-\lambda_{3,-}\right)} & e^{n\left(\lambda_{2,+}-\lambda_{3,-}\right)} & 1 & 0 \\
0 & 0 & 0 & 1
\end{array}\right) \text { on }\left(-i \widehat{\gamma}_{2}, i \widehat{\gamma}_{2}\right) \cap i \mathbb{R}^{ \pm}
$$

Case V. In Case V we have $0 \notin S\left(\mu_{1}\right), c_{2}>0$ and $c_{3}>0$ so that $\widehat{\gamma}_{2}=0$, $\gamma_{2}=0$ and $\gamma_{3}=0$. There are no exceptional intervals in this case.

\subsection{Behavior of jumps as $n \rightarrow \infty$}

Having collected all the jump matrices $J_{S}$ we may study their behavior as $n \rightarrow \infty$. It turns out that all off-diagonal entries of the form $\pm e^{n\left(\lambda_{j}-\lambda_{k}\right)}$ are such that $\operatorname{Re}\left(\lambda_{j}-\lambda_{k}\right)<0$ and therefore they are exponentially decaying as $n \rightarrow \infty$.

For $\left(J_{S}\right)_{1}$ in (7.23) we have off diagonal entries

$$
\begin{cases}e^{n\left(\lambda_{2,+}-\lambda_{1,-}\right)} & \text { on } \mathbb{R} \backslash S\left(\mu_{1}\right), \\ e^{n\left(\lambda_{1}-\lambda_{2}\right)} & \text { on the lips of the lenses around } S\left(\mu_{1}\right),\end{cases}
$$

which are indeed exponentially decaying because of (4.43) and Lemma 7.5.

For $\left(J_{S}\right)_{2}$ in (7.24) we have off diagonal entries

$$
\begin{cases}e^{n\left(\lambda_{2,+}-\lambda_{3,-}\right)} & \text { on } i \mathbb{R} \backslash S\left(\sigma_{2}-\mu_{2}\right), \\ e^{n\left(\lambda_{3}-\lambda_{2}\right)} & \text { on the lips of the lens } L_{2} \text { around } S\left(\sigma_{2}-\mu_{2}\right),\end{cases}
$$

which are exponentially decaying because of (4.44) and Lemma 7.1 . 
For $\left(J_{S}\right)_{3}$ in (7.25) we have off diagonal entries

$$
\begin{cases}e^{n\left(\lambda_{4,+}-\lambda_{3,-}\right)} & \text { on } \mathbb{R} \backslash S\left(\mu_{3}\right), \\ e^{n\left(\lambda_{3}-\lambda_{4}\right)} & \text { on the lips of the lens } L_{3} \text { around } S\left(\mu_{3}\right),\end{cases}
$$

which are exponentially decaying because of (4.45) and Lemma 7.2 .

The remaining off-diagonal entries appear in the $*$ entries in (17.22) on the special intervals $\left(-\gamma_{3}, \gamma_{3}\right)$ or $\left(-i \gamma_{2}, i \gamma_{2}\right)$ and $\left(-i \widehat{\gamma}_{2}, i \widehat{\gamma}_{2}\right)$. They are explicitly given in the formulas (7.16), (7.15), (7.26), and (7.29). It turns out that all these entries are exponentially decaying as $n \rightarrow \infty$. We will not verify all the cases here, but let us check the jump matrix $J_{S}$ on $\left(-i \widehat{\gamma}_{2}, i \widehat{\gamma}_{2}\right)$ in Case I as given in (7.26). Here there are four off diagonal entries

$$
\mp e^{n\left(\lambda_{1}-\lambda_{3,-}\right)}, \quad e^{n\left(\lambda_{2,+}-\lambda_{3,-}\right)}, \quad-e^{n\left(\lambda_{1}-\lambda_{4}\right)}, \quad \pm e^{n\left(\lambda_{2,+}-\lambda_{4}\right)} .
$$

Since we are in Case I, the inequalities from (4.44) and Lemmas 7.2 and 7.5 apply on $\left[-i \widehat{\gamma}_{2}, i \widehat{\gamma_{2}}\right]$ since this interval is contained in $i \mathbb{R} \backslash S\left(\sigma_{2}-\mu_{2}\right)$ and it belongs to the two lenses $L_{1}$ and $L_{3}$. So we have

$$
\operatorname{Re}\left(\lambda_{1}-\lambda_{2}\right) \leq 0, \quad \operatorname{Re}\left(\lambda_{2,+}-\lambda_{3,-}\right)<0, \quad \operatorname{Re}\left(\lambda_{3}-\lambda_{4}\right) \leq 0
$$

on $\left[-i \widehat{\gamma}_{2}, i \widehat{\gamma}_{2}\right]$. In fact, equality in the first and third inequalities of (7.34) holds only at 0 . Then indeed all entries in (7.33) are exponentially decaying as $n \rightarrow \infty$, uniformly on $\left[-i \widehat{\gamma}_{2}, i \widehat{\gamma}_{2}\right]$.

In the next step of the steepest descent analysis we will ignore all exponentially small entries in the jump matrices $J_{S}$. This will lead to matrices $J_{M}$ that we use as jump matrices for the so-called global parametrix. The matrices $J_{M}$ are given in 8.3 - 8.6 below. 


\section{CHAPTER 8}

\section{Global Parametrix}

\subsection{Statement of RH problem}

If we ignore all entries in the jump matrices $J_{S}$ that are exponentially small as $n \rightarrow \infty$, we find the following model Riemann-Hilbert problem for $M: \mathbb{C} \backslash(\mathbb{R} \cup i \mathbb{R}) \rightarrow$ $\mathbb{C}^{4 \times 4}$

$$
\left\{\begin{array}{l}
M \text { is analytic in } \mathbb{C} \backslash(\mathbb{R} \cup i \mathbb{R}), \\
M_{+}=M_{-} J_{M}, \quad \text { on } \mathbb{R} \cup i \mathbb{R}, \\
M(z)=\left(I+\mathcal{O}\left(z^{-1}\right)\right)\left(\begin{array}{cccc}
1 & 0 & 0 & 0 \\
0 & z^{1 / 3} & 0 & 0 \\
0 & 0 & 1 & 0 \\
0 & 0 & 0 & z^{-1 / 3}
\end{array}\right)\left(\begin{array}{cc}
1 & 0 \\
0 & A_{j}
\end{array}\right) \\
\text { as } z \rightarrow \infty \text { in the } j \text { th quadrant, }
\end{array}\right.
$$

where $J_{M}$ is given as follows. On the real line, the jump matrix has the block form

$$
J_{M}(x)=\left(\begin{array}{cc}
\left(J_{M}\right)_{1}(x) & 0 \\
0 & \left(J_{M}\right)_{3}(x)
\end{array}\right), \quad x \in \mathbb{R}
$$

with

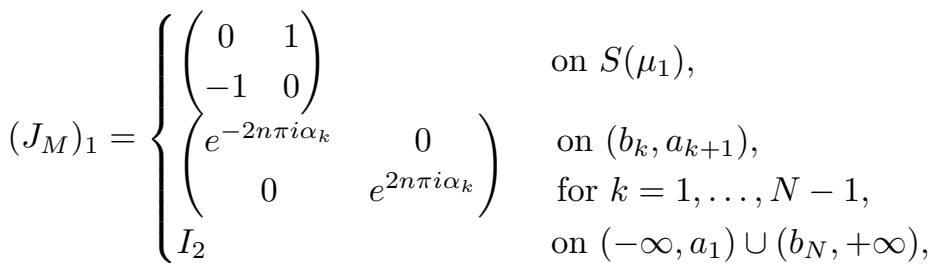

$$
\begin{aligned}
& \left(J_{M}\right)_{3}= \begin{cases}\left(\begin{array}{cc}
0 & 1 \\
-1 & 0
\end{array}\right) & \text { on } S\left(\mu_{3}\right), \\
(-1)^{n} I_{2} & \text { on }\left(-c_{3}, c_{3}\right) .\end{cases}
\end{aligned}
$$

On the imaginary axis the jump matrix has the block form

$$
J_{M}(x)=\left(\begin{array}{ccc}
1 & 0 & 0 \\
0 & \left(J_{M}\right)_{2}(x) & 0 \\
0 & 0 & 1
\end{array}\right), \quad x \in i \mathbb{R},
$$

with

$$
\left(J_{M}\right)_{2}= \begin{cases}\left(\begin{array}{cc}
0 & -1 \\
1 & 0
\end{array}\right) & \text { on } S\left(\sigma_{2}-\mu_{2}\right) \\
I_{2} & \text { on }\left(-i c_{2}, i c_{2}\right)\end{cases}
$$


Note that we have strenghtened the $\mathcal{O}$ term in the asymptotic condition in (8.1) from $\mathcal{O}\left(z^{-1 / 3}\right)$ to $\mathcal{O}\left(z^{-1}\right)$.

The solution has fourth root singularities at all branch points $a_{k}, b_{k}$, for $k=$ $1, \ldots, N$, and at $\pm c_{2}, \pm c_{3}$ if $c_{2}, c_{3}>0$. We give more details on the construction in the rest of this chapter.

\subsection{Riemann surface as an $M$-curve}

We follow the approach of 68 in using meromorphic differentials on the Riemann surface as the main ingredient in the construction of the global parametrix. Recall that $\mathcal{R}$ is a four sheeted cover of the Riemann sphere. We use

$$
\pi: \mathcal{R} \rightarrow \overline{\mathbb{C}}
$$

to denote the canonical projection, and we let $\pi_{j}$ be its restriction to the $j$ th sheet. Then for $z \in \mathbb{C}$ we have that

$$
\pi_{j}^{-1}(z)
$$

denotes the point on the $j$ th sheet that projects onto $z$. It is well-defined for $z \in \mathbb{C} \backslash(\mathbb{R} \cup i \mathbb{R})$.

The Riemann surface $\mathcal{R}$ has the structure of an $M$-curve. It has an antiholomorphic involution

$$
\phi: \mathcal{R} \rightarrow \mathcal{R}: P \mapsto \bar{P}
$$

with $\bar{P}$ on the same sheet as $P$. The fixed point set of $\phi$ consists of $g+1$ connected components ( $g$ is the genus of $\mathcal{R}$ )

$$
\{P \in \mathcal{R}: \phi(P)=P\}=\Sigma_{0} \cup \Sigma_{1} \cup \cdots \cup \Sigma_{g}
$$

where

- $\Sigma_{0}$ contains $\infty_{1}$ and consists of the intervals $\left(-\infty, a_{1}\right]$ and $\left[b_{N}, \infty\right)$ on the first and second sheets.

- $\Sigma_{i}$ for $i=1, \ldots, N-1$ contains the intervals $\left[b_{i}, a_{i+1}\right]$ on the first and second sheets.

A full description of the curves depends on the case we are in. Recall that there are five cases.

Cases I and II: In Cases I and II we have $c_{3}=0$ and the genus is $N-1$. In these cases we have only the above curves $\Sigma_{0}, \ldots, \Sigma_{N-1}$ and they are on the first and second sheets only.

Case III: In Case III we still have $g=N-1$. Since $0 \notin \operatorname{supp}\left(\mu_{1}\right)$ the number $N$ is even (by symmetry). The curve $\Sigma_{N / 2}$ is now of a different character, since it visits all four sheets. Indeed we have that $\Sigma_{N / 2}$ consists of $\left[b_{N / 2}, a_{N / 2+1}\right]$ on the first and second sheets, and $\left[-c_{3}, c_{3}\right]$ on the third and fourth seets. The component $\Sigma_{N / 2}$ is also unusual in that it contains four branch points, in contrast to the other curves that have two branch points.

Cases IV and V: In Cases IV and V the genus is $N$. In these cases there is an additional component $\Sigma_{N}$ that consists of the intervals $\left[-c_{3}, c_{3}\right]$ on the third and fourth sheets. The other components are on the first and second sheets only, as in Cases I and II.

We need the following result on non-special divisors on $M$-curves. 


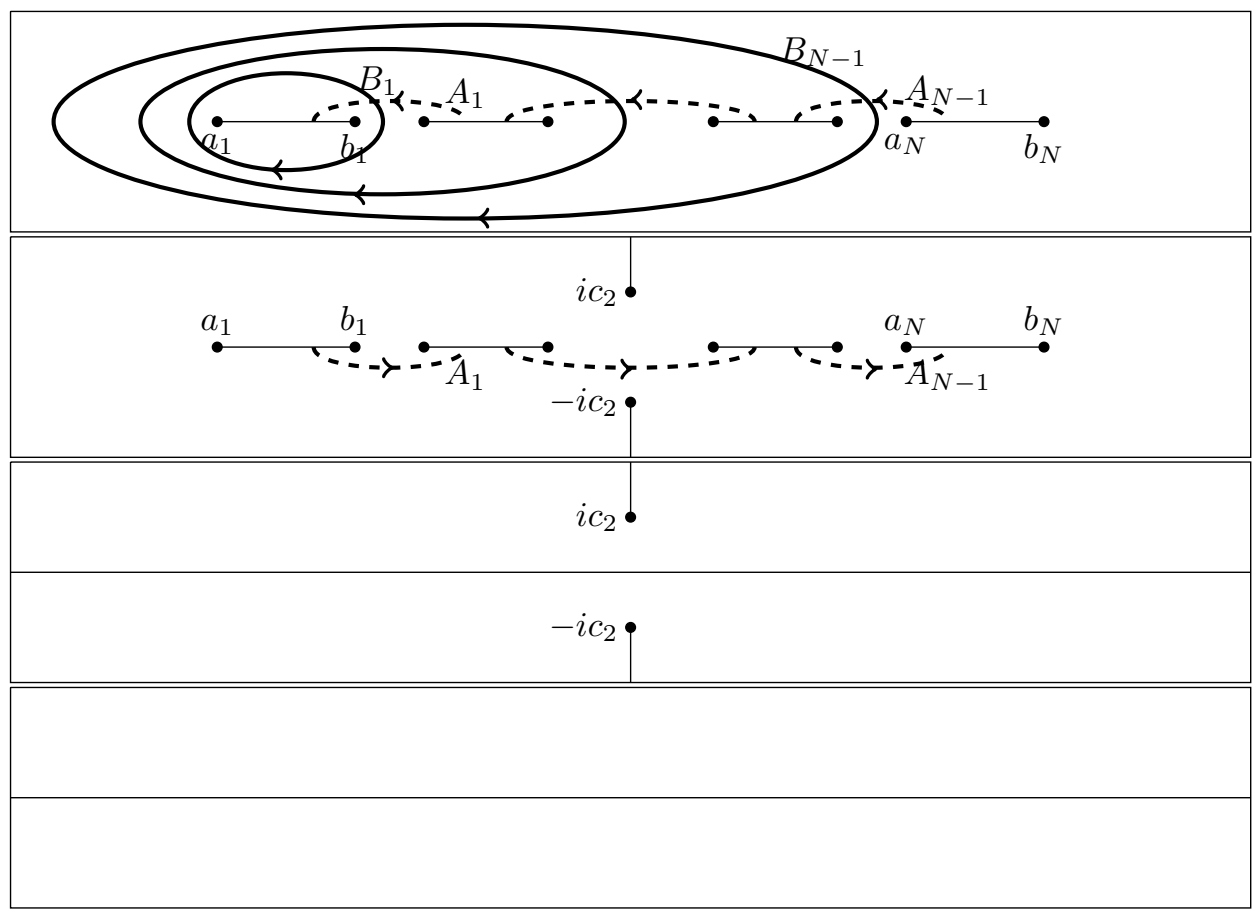

Figure 8.1. Canonical homology basis in Case II

Lemma 8.1. If $P_{j} \in \Sigma_{j}$ for $j=1, \ldots, g$ then the divisor $\sum_{j=1}^{g} P_{j}$ is non-special.

Proof. This can be found in [58, Theorem 2.4].

Recall that the divisor $\sum_{j=1}^{g} P_{j}$ is non-special if and only if there are no nonconstant analytic functions on $\mathcal{R} \backslash\left\{P_{1}, \ldots, P_{g}\right\}$ with only simple poles at the points $P_{1}, \ldots, P_{g}$.

\subsection{Canonical homology basis}

The Riemann surface has a canonical homology basis $\left\{A_{1}, \ldots, A_{g} ; B_{1}, \ldots, B_{g}\right\}$ that we choose such that the cycle $A_{j}$ is homologous to $\Sigma_{j}$ but disjoint from it. See Figures 8.18 .3 for an illustration of the curves for the cycles with their orientation in the various cases. In all cases we choose the $B$-cycles such that as sets they are invariant under the involution $\phi$.

The Cases I and II are very similar and we only show the Figure 8.1 for Case II. The $B$-cycles are on the first sheet only. The cycle $B_{j}$ surrounds the interval $\left[a_{1}, b_{j}\right]$ once in the negative (clockwise) direction, and it intersects the real line in $\left(-\infty, a_{1}\right)$ and in $\left(b_{j}, a_{j+1}\right)$. The $A$-cycles are partly on the upper half of the first sheet and partly on the lower half of the second sheet. The cycle $A_{j}$ passes through the cuts $\left[a_{j}, b_{j}\right]$ and $\left[a_{j+1}, b_{j+1}\right]$ with orientation as indicated in Figure 8.1. In Case II there is an $A$-cycle that intersects with the imaginary axis. We make sure that on the second sheet it does so in the interval $\left(-i c_{2}, 0\right)$. 


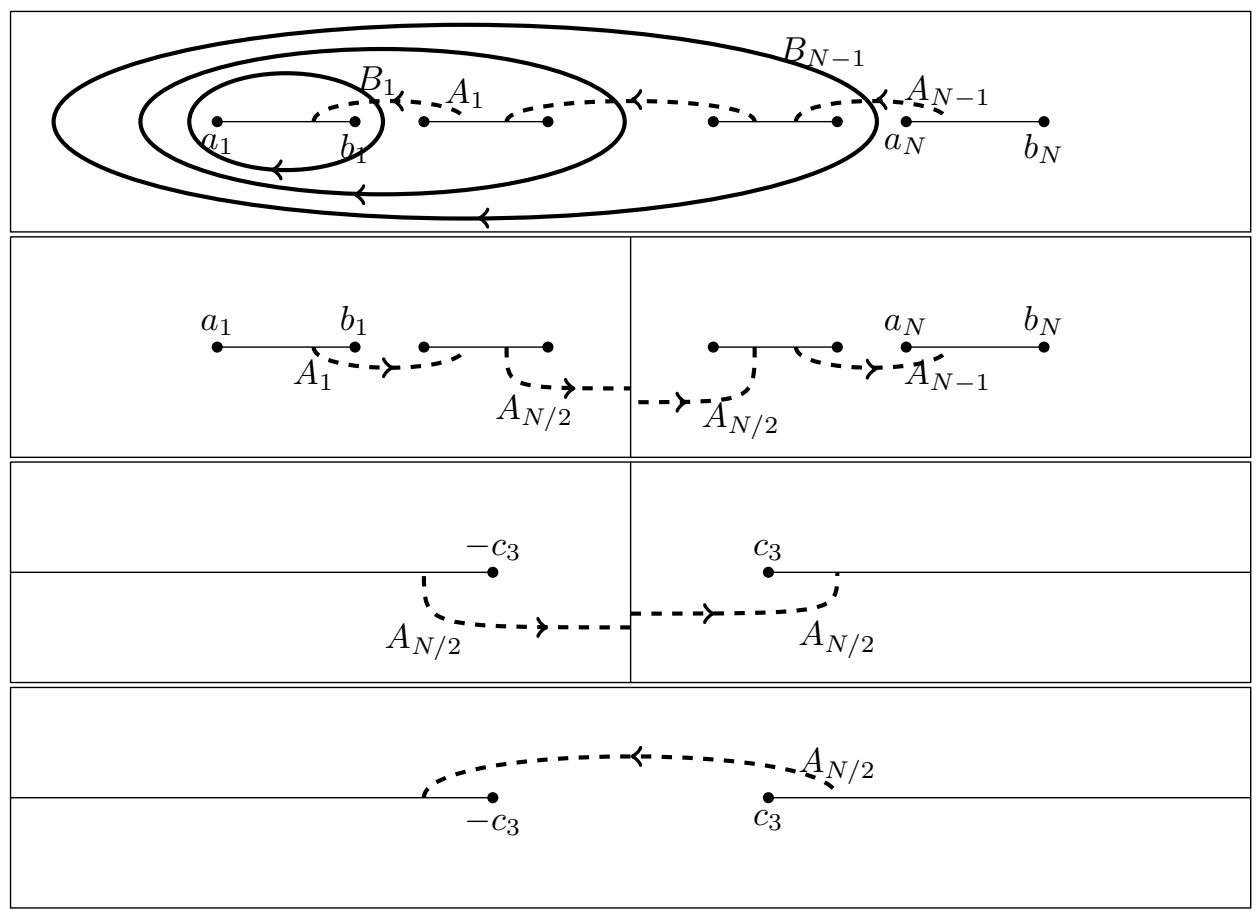

Figure 8.2. Canonical homology basis in Case III

In Case III we have $c_{2}=0$ and $c_{3}>0$. The genus of $\mathcal{R}$ is $g=N-1$. Note that in this case, the number of intervals in $S\left(\mu_{1}\right)$ is even and the origin does not belong to $S\left(\mu_{1}\right)$. The canonical homology basis is chosen as in Figure 8.2. The $B$-cycles are the same as in Case II. In particular they are only on the first sheet. The $A$-cylces are also the same as in Case II, except for the cycle $A_{N / 2}$ which crosses the imaginary axis. This cycle visits all four sheets as indicated in Figure 8.2 .

The two Cases IV and V are again very similar and we only show the Figure 8.3 for Case $\mathrm{V}$. Here we have $c_{2}>0$ and $c_{3}>0$, and the Riemann surface $\mathcal{R}$ has genus $g=N$. The cycles $A_{1}, \ldots, A_{N-1}$ and $B_{1}, \ldots, B_{N-1}$ are as in the previous Cases I and II. There are two extra cycles $A_{N}$ and $B_{N}$. The cycle $A_{N}$ is on the third and fourth sheets. It consists of a part in the upper half plane of $\mathcal{R}_{3}$ from a point in $\left(c_{3}, \infty\right)$ to a point in $\left(-\infty,-c_{3}\right)$, together with a part in the lower half plane of $\mathcal{R}_{4}$ that we choose to be the mirror image in the real line of the part on the third sheet. The cycle $B_{N}$ is on the second and third sheets. The part on $\mathcal{R}_{2}$ goes from a point in $\left(-i \infty,-i c_{2}\right)$ to a point in $\left(i c_{2}, \infty\right)$ and it goes around all intervals $\left[a_{j}, b_{j}\right]$ that are in the left-half plane. The part on $\mathcal{R}_{3}$ intersect the real line somewhere in $\left(0, c_{3}\right)$.

By construction we have

$$
\phi\left(B_{j}\right)=-B_{j}, \quad \phi\left(A_{j}\right) \sim A_{j}, \quad j=1, \ldots, g
$$

where the symbol $\sim$ means that $\phi\left(A_{j}\right)$ is homologous to $A_{j}$ in $\mathcal{R}$. 


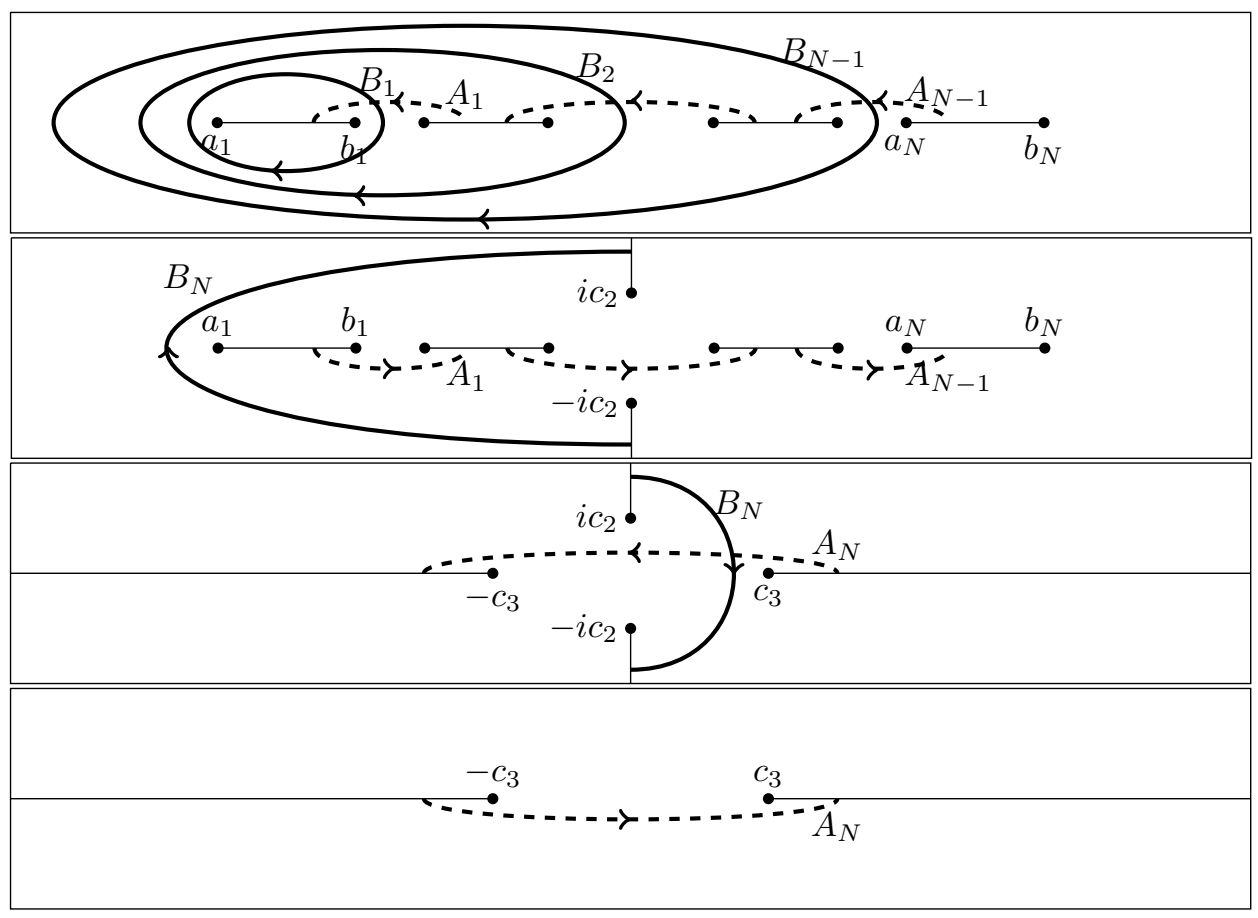

Figure 8.3. Canonical homology basis in Case V

\subsection{Meromorphic differentials}

Let us now recall some facts about meromorphic differentials on the Riemann surface. Most of the results that we will be using can be found in $\mathbf{5 3}$.

A meromorphic differential with simple poles only is called a meromorphic differential of the third kind. A meromorphic differential of the third kind is uniquely determined by its $A$-periods and the location and residues at its poles, provided that the residues add up to zero.

We pick points $P_{j}, j=1, \ldots, g$ with $P_{j} \in \Sigma_{j}$. For each such choice we define a meromorphic differential $\omega_{P}$ of the third kind as follows.

We use $\infty_{1}$ to denote the point at infinity on the first sheet and $\infty_{2}$ to denote the other point at infinity which is common to all three other sheets.

Definition 8.2. Let $P_{j} \in \Sigma_{j}$ for $j=1, \ldots, g$. Then $\omega_{P}$ is the meromorphic differential of third kind on $\mathcal{R}$ which is uniquely determined by the following conditions

(a) The meromorphic differential has simple poles at $a_{j}, b_{j}, j=1, \ldots, N$, at $\pm i c_{2}$ (if $c_{2}>0$ ), at $\pm c_{3}$ (if $c_{3}>0$ ), at the points $P_{j}, j=1, \ldots, g$ and at $\infty_{2}$. The residues at the finite branch points are equal to $-1 / 2$ :

$$
\begin{aligned}
\operatorname{Res}_{z=a_{j}} \omega_{P} & =\operatorname{Res}_{z=b_{j}} \omega_{P}=-\frac{1}{2}, \quad j=1, \ldots, N, \\
\operatorname{Res}_{z= \pm i c_{2}} \omega_{P} & =-\frac{1}{2}, \quad\left(\text { only if } c_{2}>0\right), \\
\operatorname{Res}_{z= \pm c_{3}} \omega_{P} & \left.=-\frac{1}{2}, \quad \text { (only if } c_{3}>0\right),
\end{aligned}
$$


the residue at $\infty_{2}$ is equal to 2 ,

$$
\operatorname{Res}_{z=\infty_{2}} \omega_{P}=2
$$

and the residue at the points $P_{j}$ is equal to 1 :

$$
\underset{z=P_{j}}{\operatorname{Res}} \omega_{P}=1, \quad j=1, \ldots, g .
$$

(b) The meromorphic differential has vanishing $A$ periods in Cases I, II, IV, and V:

$$
\oint_{A_{k}} \omega_{P}=0, \quad k=1, \ldots, g, \quad \text { (in Cases I, II, IV, and V). }
$$

In Case III all $A$-periods are vanishing, except the one of $A_{N / 2}$ :

$$
\oint_{A_{k}} \omega_{P}=-\pi i \delta_{k, N / 2}, \quad k=1, \ldots, g, \quad \text { (only in Case III). }
$$

A simple count shows that the residues of $\omega_{P}$ add up to 0 and therefore the meromorphic differential $\omega_{P}$ is indeed uniquely defined by the pole conditions and the $A$-period conditions.

If one or more of the $P_{j}$ 's coincide with a branch point, then the residue conditions (8.8) have to be modified appropriately. For example, if $P_{j}=a_{j}$ then

$$
\operatorname{Res}_{P_{j}} \omega_{P}=\frac{1}{2} \text {. }
$$

In this way, the meromorphic differential $\omega_{P}$ depends continuously on the $P_{j}$ 's and is well defined for each choice of $P_{j} \in \Gamma_{j}, j=1, \ldots, g$.

The anti-holomorphic involution $\phi$ is used to map a meromorphic differential $\omega$ to a meromorphic differential $\phi^{\#}(\omega)$ in an obvious way. If $\omega$ is equal to $f_{j}(z) d z$ for a meromorphic function $f_{j}$ on sheet $j$, then $\phi^{\#}(\omega)$ is equal to

$$
\overline{f_{j}(\bar{z})} d z
$$

on sheet $j$. A crucial property is that $\omega_{P}$ is invariant under $\phi^{\#}$.

Lemma 8.3. Let $P_{j} \in \Sigma_{j}, j=1, \ldots, g$. Then

$$
\omega_{P}=\phi^{\#}\left(\omega_{P}\right) \text {. }
$$

Proof. Since all the poles of $\omega_{P}$ are invariant under the involution $\phi$, the meromorphic differential $\phi^{\#}\left(\omega_{P}\right)$ has the same poles and residues as $\omega_{P}$. We have to show that their $A$-periods are the same. We have

$$
\oint_{A_{k}} \phi^{\#}\left(\omega_{P}\right)=\overline{\oint_{\phi\left(A_{k}\right)} \omega_{P}}
$$

We have that $\phi\left(A_{k}\right)$ is homologous to $A_{k}$ in $\mathcal{R}$, but in the process of deforming $\phi\left(A_{k}\right)$ to $A_{k}$ we pick up residue contributions from the poles of $\omega_{P}$.

For the cycles $A_{k}$ that are only on two sheets (which is the typical situation) we pick up a residue contribution from the two endpoints of a gap in the support of either $\mu_{1}$ or $\mu_{3}$ and from $P_{k}$. As the deformation from $\phi\left(A_{k}\right)$ to $A_{k}$ will result in clockwise loops around these points, the total residue is $\frac{1}{2}+\frac{1}{2}-1=0$. It follows that

$$
\oint_{A_{k}} \phi^{\#}\left(\omega_{P}\right)=\overline{\oint_{A_{k}} \omega_{P}}=0
$$

since the $A$-period of $\omega_{P}$ is zero for $A$-cycles that are only on two sheets. 
In Case III there is a cycle $A_{N / 2}$ which is on all four sheets. If we deform $\phi\left(A_{N / 2}\right)$ into $A_{N / 2}$ we pick up residue contributions from the endpoints $b_{N / 2}, a_{N / 2+1},-c_{3}, c_{3}$ and from $P_{N / 2}$. Then we have residue $\frac{1}{2}$ four times and -1 once, so that the total residue contribution is 1 (As the residue contribution comes from clockwise loops around these points). It follows that

$$
\oint_{A_{N / 2}} \phi^{\#}\left(\omega_{P}\right)=\overline{\oint_{A_{N / 2}} \omega_{P}+2 \pi i}=\overline{-\pi i+2 \pi i}=-\pi i,
$$

since the $A_{N / 2}$ period is $-\pi i$ by definition (8.12).

So the $A$-periods of $\phi^{\#}\left(\omega_{P}\right)$ and $\omega_{P}$ agree, and the lemma follows.

Proposition 8.4. The B-periods of $\omega_{P}$ are purely imaginary and the map

$$
\begin{aligned}
\Psi: \Sigma_{1} \times \cdots \times \Sigma_{g} & \rightarrow(\mathbb{R} / \mathbb{Z})^{g}: \\
\left(P_{1}, \ldots, P_{g}\right) & \mapsto \frac{1}{2 \pi i}\left(\int_{B_{1}} \omega_{P}, \ldots, \int_{B_{g}} \omega_{P}\right)
\end{aligned}
$$

is a well-defined, continuous bijection.

Proof. This follows as in 68. The fact that the map is well-defined and continuous is proved as in [68, Proposition 2.3]. Due to the fact that the divisors $\sum_{j=1}^{g} P_{j}$ with $P_{j} \in \Sigma_{j}$ are non-special, see Lemma 8.1, the argument in the proof of [68. Theorem 2.6] gives first the injectivity of $\Psi$. Then the invariance of domain argument of the same proof yields the surjectivity of $\Psi$.

\subsection{Definition and properties of functions $u_{j}$}

Due to Proposition 8.4 there exists a choice of points $P_{j}, j=1, \ldots, g$ such that the corresponding meromorphic differential $\omega_{P}$ satisfies

$$
\begin{aligned}
& \oint_{B_{k}} \omega_{P}=-2 n \pi i \alpha_{k}, \quad k=1, \ldots, N-1, \\
& \oint_{B_{N}} \omega_{P}=-n \pi i, \quad(\text { only in Cases IV and V), }
\end{aligned}
$$

where the equalities hold modulo $2 \pi i \mathbb{Z}$. Note that $\omega_{P}$ is varying with $n$. We consider $n$ as fixed and work with $\omega_{P}$ satisfying (8.15) throughout this chapter. Of course, $\omega_{P}$ also satisfies the conditions given in Definition 8.2

We are going to integrate $\omega_{P}$ along paths on $\mathcal{R}$ that start from $\infty_{1}$ (the point at infinity on the first sheet) and that on each sheet remain in the same quadrant. So the paths do not cross the contours $\Sigma_{j}, j=0, \ldots, g$ and also do not intersect the imaginary axis except along the cut $S\left(\sigma_{2}-\mu_{2}\right)$ that connects the second and third sheets. There may be a choice in the cut that one takes when passing from the first to the second sheet. However, this will lead to the same value for the integral because of the vanishing of the integral of $\omega_{P}$ over the cycles $A_{k}$ and $\phi\left(A_{k}\right)$, see (8.11). Note that the exceptional $A$-period (8.12) in Case III does not play a role here.

With this convention for the paths we define functions $u_{j}$ as follows. 
Definition 8.5. For $j=1,2,3,4$ and $z \in \mathbb{C} \backslash(\mathbb{R} \cup i \mathbb{R})$ we define $u_{j}(z)$ as the Abelian integral

$$
u_{j}(z)=\int_{\infty_{1}}^{\pi_{j}^{-1}(z)} \omega_{P} .
$$

Abusing the notation, we also write

$$
u_{j}(z)=\int_{\infty_{1}}^{z} \omega_{P}
$$

where $z$ is considered as a point on the $j$ th sheet. The path from $\infty_{1}$ to $z \in \mathbb{R}_{j}$ follows the convention described above, namely that on each sheet it stays in the same quadrant.

Then the functions $u_{j}$ are defined and analytic on $\mathbb{C} \backslash(\mathbb{R} \cup i \mathbb{R})$ with the following jump properties. In what follows we write $\equiv$ to denote equality up to integer multiples of $2 \pi i$.

Lemma 8.6. (a) For $x \in\left(a_{k}, b_{k}\right)$ with $k=1, \ldots, N$, we have

$$
u_{1, \pm}(x)=u_{2, \mp}(x) .
$$

(b) For $x \in\left(b_{k}, a_{k+1}\right)$ with $k=0, \ldots, N$, we have

$$
\begin{aligned}
& u_{1,+}(x) \equiv u_{1,-}(x)-2 n \pi i \alpha_{k}, \\
& \text { unless } P_{k} \text { is on the first sheet and } x=\pi\left(P_{k}\right) \\
& u_{2,+}(x) \equiv u_{2,-}(x)+2 n \pi i \alpha_{k}+\pi i, \\
& \text { unless } P_{k} \text { is on the second sheet and } x=\pi\left(P_{k}\right)
\end{aligned}
$$

where $b_{0}=-\infty, a_{N+1}=+\infty, \alpha_{0}=1$ and $\alpha_{N}=0$.

(c) For $x \in\left(-c_{3}, c_{3}\right)$ we have

$$
\begin{array}{r}
u_{3,+}(x) \equiv u_{3,-}(x)-(n+1) \pi i \\
\text { unless } P_{k} \text { is on the third sheet and } x=\pi\left(P_{k}\right) \\
u_{4,+}(x) \equiv u_{4,-}(x)+n \pi i, \\
\text { unless } P_{k} \text { is on the fourth sheet and } x=\pi\left(P_{k}\right)
\end{array}
$$

where $k=N / 2$ in Case III and $k=N$ in Cases $I V$ and $V$.

(d) For $x \in\left(-\infty,-c_{3}\right) \cup\left(c_{3}, \infty\right)$ we have

$$
u_{3, \pm}(x)=u_{4, \mp}(x) \text {. }
$$

(e) On the imaginary axis we have

$$
\begin{aligned}
& u_{1,+}(x)=u_{1,-}(x) \quad \text { for } x \in i \mathbb{R} \text {, } \\
& u_{2,+}(x)=u_{2,-}(x) \quad \text { for } x \in\left(-i c_{2}, i c_{2}\right) \text {, } \\
& u_{2, \pm}(x)=u_{3, \mp}(x) \quad \text { for } x \in\left(-i \infty,-i c_{2}\right] \cup\left[i c_{2}, i \infty\right) \text {, } \\
& u_{3,+}(x) \equiv u_{3,-}(x)+\pi i \quad \text { for } x \in\left(-i c_{2}, i c_{2}\right) \text {, } \\
& u_{4,+}(x) \equiv u_{4,-}(x)+\pi i \quad \text { for } x \in i \mathbb{R} .
\end{aligned}
$$

Proof. This is a straightforward but rather tedious verification. All identities or equivalences come down to the calculation of a period of a closed loop on $\mathcal{R}$ for the meromorphic differential $\omega_{P}$. 


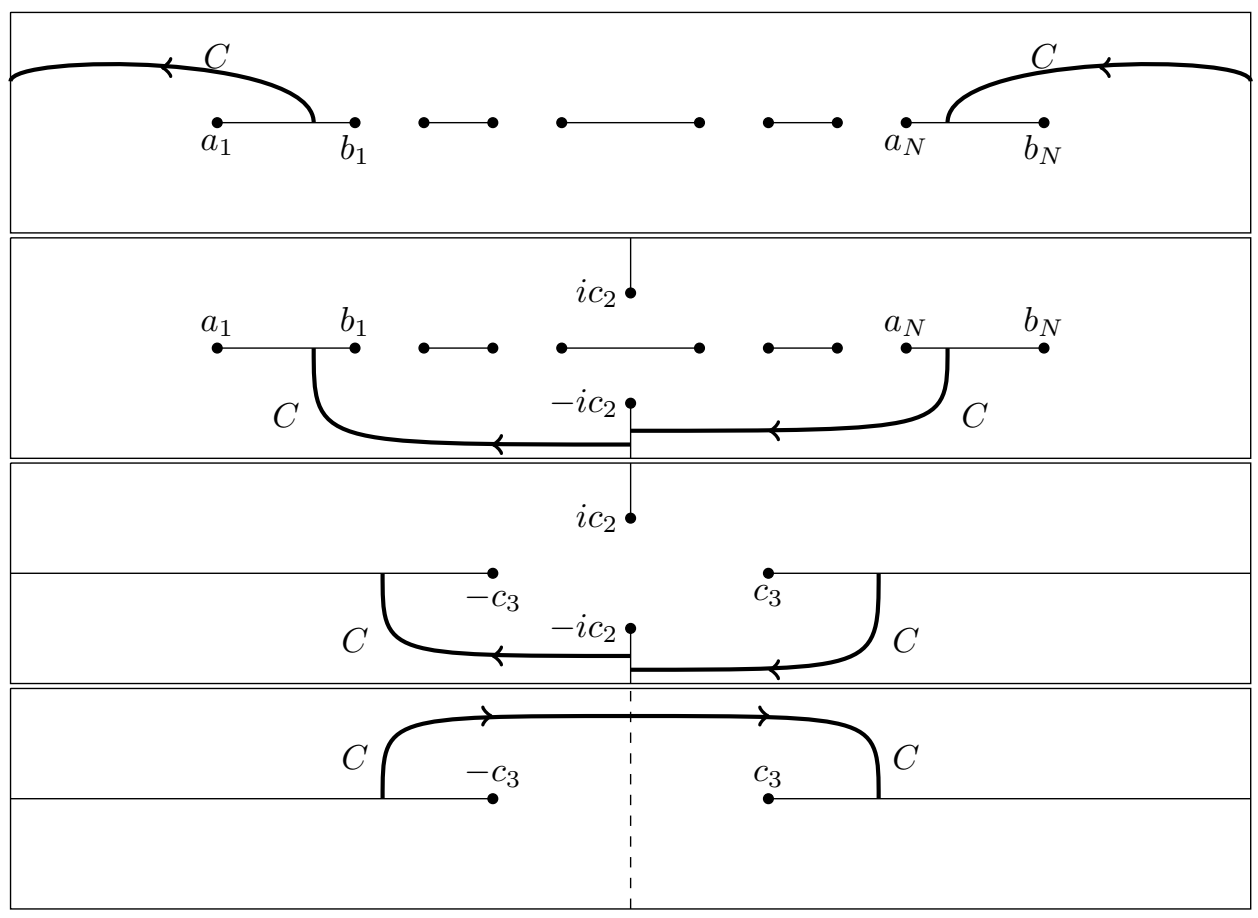

Figure 8.4. Cycle $C$ for the cases IV and V that appear in the proof of Lemma 8.6

For example, to prove (8.26) for $x \in i \mathbb{R}^{+}$, we note that

$$
u_{4,+}(x)-u_{4,-}(x)=\int_{C} \omega_{P}
$$

where $C$ is the cycle on $\mathcal{R}$ that is shown in Figure 8.4 for the Cases IV and V. This cycle can be deformed to a sum of $-\phi\left(A_{N}\right)$, the cycles $-A_{j}, j=1, \ldots, N-1$ and a closed loop around $-i c_{2}$. This indeed leads to (8.26) since $\omega_{P}$ has vanishing $A$ periods and $\phi(A)$ periods, and the only contribution comes from $-i c_{2}$ which gives us $\pm \pi i$.

In the Case III (not shown in the figure), the cycle $C$ can be deformed into the cycles, $-A_{j}, j=1, \ldots N-1$, that include the exceptional cycle $-A_{N / 2}$. It is now because of (8.12) that we obtain (8.26) for $x \in i \mathbb{R}^{+}$.

The other relations follows in a similar way.

We state without proof the behavior of the functions $u_{j}$ near the branch points. They follow from residue conditions (8.8).

LEMMA 8.7.

(a) For $j=1,2$ and $k=1, \ldots, N$, we have

$$
\begin{aligned}
& u_{j}(z)=-\frac{1}{4} \log \left(z-a_{k}\right)+\mathcal{O}(1), \quad \text { as } z \rightarrow a_{k}, \\
& u_{j}(z)=-\frac{1}{4} \log \left(z-b_{k}\right)+\mathcal{O}(1), \quad \text { as } z \rightarrow b_{k} .
\end{aligned}
$$

(b) For $j=2,3$ and $c_{2}>0$, we have

$$
u_{j}(z)=-\frac{1}{4} \log \left(z \mp i c_{2}\right)+\mathcal{O}(1), \quad \text { as } z \rightarrow \pm i c_{2} .
$$


(c) For $j=3,4$ and $c_{3}>0$, we have

$$
u_{j}(z)=-\frac{1}{4} \log \left(z \mp c_{3}\right)+\mathcal{O}(1), \quad \text { as } z \rightarrow \pm c_{3} .
$$

(d) If $P_{k}$ is on the jth sheet of the Riemann surface, then

$$
u_{j}(z)=\log \left(z-\pi\left(P_{k}\right)\right)+\mathcal{O}(1), \quad \text { as } z \rightarrow \pi\left(P_{k}\right),
$$

where $k=1, \ldots, g$.

(e) As $z \rightarrow \infty$,

$$
u_{1}(z)=\mathcal{O}(1 / z) \quad \text { and } \quad u_{j}(z)=-\frac{2}{3} \log z+\mathcal{O}(1), \quad \text { for } j=2,3,4 \text {. }
$$

If $P_{k}$ coincides with one of the branch points then the behavior should be modified. This should be obvious and we do not give details here.

\subsection{Definition and properties of functions $v_{j}$}

The functions $v_{j}$ are the exponentials of the functions $u_{j}$ which we again consider as functions on $\mathbb{C} \backslash(\mathbb{R} \cup i \mathbb{R})$.

Definition 8.8. For $j=1,2,3,4$ and $z \in \mathbb{C} \backslash(\mathbb{R} \cup i \mathbb{R})$ we define

$$
v_{j}(z)=e^{u_{j}(z)}
$$

The jump properties of $v_{j}$ follow from Lemma 8.6. We state them in a vector form.

COROLLARY 8.9. The vector-valued function $\left(\begin{array}{llll}v_{1} & v_{2} & v_{3} & v_{4}\end{array}\right)$ is analytic in $\mathbb{C} \backslash(\mathbb{R} \cup i \mathbb{R})$ with jump property

$$
\left(\begin{array}{llll}
v_{1} & v_{2} & v_{3} & v_{4}
\end{array}\right)_{+}=\left(\begin{array}{llll}
v_{1} & v_{2} & v_{3} & v_{4}
\end{array}\right)_{-} J_{v} \quad \text { on } \mathbb{R} \cup i \mathbb{R}
$$

with a jump matrix $J_{v}$ that takes the following form.

(a) On the real axis the jump matrix has the block form

$$
J_{v}=\left(\begin{array}{cc}
\left(J_{v}\right)_{1} & 0 \\
0 & \left(J_{v}\right)_{3}
\end{array}\right) \quad \text { on } \mathbb{R}
$$

with $2 \times 2$ blocks

$$
\begin{array}{ll}
\left(J_{v}\right)_{1}=\left(\begin{array}{ll}
0 & 1 \\
1 & 0
\end{array}\right) & \text { on } S\left(\mu_{1}\right), \\
\left(J_{v}\right)_{1}=\left(\begin{array}{cc}
e^{-2 n \pi i \alpha_{k}} & 0 \\
0 & -e^{2 n \pi i \alpha_{k}}
\end{array}\right) & \text { on }\left(b_{k}, a_{k+1}\right),
\end{array}
$$

for $k=0, \ldots, N$, and

$$
\begin{array}{ll}
\left(J_{v}\right)_{3}=\left(\begin{array}{ll}
0 & 1 \\
1 & 0
\end{array}\right) & \text { on } S\left(\mu_{3}\right), \\
\left(J_{v}\right)_{3}=\left(\begin{array}{cc}
(-1)^{n+1} & 0 \\
0 & (-1)^{n}
\end{array}\right) & \text { on }\left(-c_{3}, c_{3}\right) .
\end{array}
$$

(b) On the imaginary axis the jump matrix has the block form

$$
J_{v}=\left(\begin{array}{ccc}
1 & 0 & 0 \\
0 & \left(J_{v}\right)_{2} & 0 \\
0 & 0 & -1
\end{array}\right) \quad \text { on } i \mathbb{R}
$$


with the $2 \times 2$ block

$$
\begin{array}{ll}
\left(J_{v}\right)_{2}=\left(\begin{array}{cc}
0 & 1 \\
1 & 0
\end{array}\right) & \text { on } S\left(\sigma_{2}-\mu_{2}\right), \\
\left(J_{v}\right)_{2}=\left(\begin{array}{cc}
1 & 0 \\
0 & -1
\end{array}\right) & \text { on }\left(-i c_{2}, i c_{2}\right) .
\end{array}
$$

Note that each of the blocks $\left(J_{v}\right)_{j}, j=1,2,3$ has determinant -1 .

From Lemma 8.7 and Definition 8.8 we obtain the behavior of the $v_{j}$ functions near the branch points and other special points.

Corollary 8.10. $\quad$ (a) For $j=1,2$ and $k=1, \ldots, N$, we have

$$
\begin{aligned}
& v_{j}(z)=\mathcal{O}\left(\left(z-a_{k}\right)^{-1 / 4}\right), \quad \text { as } z \rightarrow a_{k}, \\
& u_{j}(z)=\mathcal{O}\left(\left(z-b_{k}\right)^{-1 / 4}\right), \quad \text { as } z \rightarrow b_{k} .
\end{aligned}
$$

(b) For $j=2,3$ and $c_{2}>0$, we have

$$
u_{j}(z)=\mathcal{O}\left(\left(z \mp i c_{2}\right)^{-1 / 4}\right), \quad \text { as } z \rightarrow \pm i c_{2} .
$$

(c) For $j=3,4$ and $c_{3}>0$, we have

$$
u_{j}(z)=\mathcal{O}\left(\left(z \mp c_{3}\right)^{-1 / 4}\right), \quad \text { as } z \rightarrow \pm c_{3} .
$$

(d) If $P_{k}$ is on the $j$ th sheet of the Riemann surface, then

$$
v_{j}(z)=\mathcal{O}\left(z-\pi\left(P_{k}\right)\right), \quad \text { as } z \rightarrow \pi\left(P_{k}\right),
$$

where $k=1, \ldots, g$.

(e) $A s z \rightarrow \infty$,

$$
v_{1}(z)=1+\mathcal{O}(1 / z) \quad \text { and } \quad v_{j}(z)=\mathcal{O}\left(z^{-2 / 3}\right), \quad \text { for } j=2,3,4 .
$$

\subsection{The first row of $M$}

We now define the entries in the first row of $M$.

Definition 8.11. We define

$$
\begin{aligned}
& M_{11}(z)=v_{1}(z), \\
& M_{12}(z)= \begin{cases}v_{2}(z) & \text { for } \operatorname{Im} z>0, \\
-v_{2}(z) & \text { for } \operatorname{Im} z<0,\end{cases} \\
& M_{13}(z)= \begin{cases}v_{3}(z) & \text { for } z \text { in the first and third quadrants, } \\
-v_{3}(z) & \text { for } z \text { in the second and fourth quadrants, }\end{cases} \\
& M_{14}(z)= \begin{cases}-v_{4}(z) & \text { for } \operatorname{Re} z>0, \\
v_{4}(z) & \text { for } \operatorname{Re} z<0 .\end{cases}
\end{aligned}
$$

It turns out that with this distribution of \pm signs the row vector $\left(M_{1 j}\right)$ has exactly the correct jump properties that are required in the $\mathrm{RH}$ problem for $M$. 
Proposition 8.12. The row vector $\left(M_{1 j}\right)_{j=1, \ldots 4}$ satisfies the conditions that are necessary for the first row of the solution of the $R H$ problem for $M$.

That is, the entries $M_{1 j}$ are analytic in $\mathbb{C} \backslash(\mathbb{R} \cup i \mathbb{R})$ with jump property

$$
\left(\begin{array}{llll}
M_{11} & M_{12} & M_{13} & M_{14}
\end{array}\right)_{+}=\left(\begin{array}{llll}
M_{11} & M_{12} & M_{13} & M_{14}
\end{array}\right)_{-} J_{M}
$$

on $\mathbb{R} \cup i \mathbb{R}$, where $J_{M}$ is the jump matrix in the $R H$ problem for $M$, see (8.1), and also

$$
\begin{aligned}
&\left(\begin{array}{lllll}
M_{11}(z) & M_{12}(z) & M_{13}(z) & M_{14}(z)
\end{array}\right) \\
&=\left(\begin{array}{llll}
1+\mathcal{O}\left(z^{-1}\right) & \mathcal{O}\left(z^{-2 / 3}\right) & \mathcal{O}\left(z^{-2 / 3}\right) & \mathcal{O}\left(z^{-2 / 3}\right)
\end{array}\right)
\end{aligned}
$$

as $z \rightarrow \infty$.

Proof. This follows directly from Lemma 8.9, Corollary 8.10 (e), and Definition 8.11

\subsection{The other rows of $M$}

We will now construct the other rows of $M$ out of the first row.

LEMMA 8.13. The vector space of meromorphic functions on $\mathcal{R}$ (including the constant functions) whose divisor is greater than or equal to

$$
-\sum_{j=1}^{g} P_{j}-3 \infty_{2}
$$

is of dimension 4 .

Proof. Let us denote, for a positive divisor $D^{\prime}$, the space of meromorphic functions on $\mathcal{R}$ whose divisor is greater than or equal to $-D^{\prime}$ by $L\left(D^{\prime}\right)$.

Let $D=\sum_{j=1}^{g} P_{j}$. Any $F \in L\left(D+3 \infty_{2}\right)$ has a pole at $\infty_{2}$ of order at most 3 . Therefore, if $w$ is a local coordinate near $\infty_{2}$, we have

$$
F(w)=f_{3} w^{-3}+f_{2} w^{-2}+f_{1} w^{-1}+\mathcal{O}(1) \quad \text { as } w \rightarrow 0,
$$

for certain numbers $f_{1}, f_{2}, f_{3}$. The kernel of the linear map

$$
L\left(D+3 \infty_{2}\right) \rightarrow \mathbb{C}^{3}: f \mapsto\left(f_{1}, f_{2}, f_{3}\right)
$$

is equal to the space $L(D)$. Since $D$ is non-special, the space $L(D)$ contains only constant functions and therefore is of dimension 1 . Therefore by the dimension theorem for linear maps

$$
\operatorname{dim} L\left(D+3 \infty_{2}\right) \leq \operatorname{dim} L(D)+3=4 .
$$

However, by the Riemann-Roch theorem (see e.g. [53]), we also have

$$
L\left(D+3 \infty_{2}\right) \geq 4 .
$$

This proves the lemma.

We continue to use

$$
D=\sum_{j=1}^{g} P_{j} .
$$

Let $F_{j}$ be a function in $L\left(D+3 \infty_{2}\right)$. We use $F_{j k}$ to denote the restriction of $F_{j}$ to the $k$ th sheet and we consider the row vector

$$
\left(\begin{array}{llll}
M_{11} F_{j 1} & M_{12} F_{j 2} & M_{13} F_{j 3} & M_{14} F_{j 4}
\end{array}\right)
$$


Note that the possible poles of $F_{j k}$ at the points $\pi\left(P_{l}\right)$ are cancelled by the zeros of $M_{1 k}$ at the same point. Therefore the row vector remains bounded at these points and it has the same behavior near the branch points as the first row.

It is easy to check that the row vector has a jump on $\mathbb{R} \cup i \mathbb{R}$ with jump matrix $J_{M}$. In order to construct the other rows we therefore aim to find three functions $F_{2}, F_{3}$ and $F_{4}$ in $L\left(D+3 \infty_{2}\right)$ such that the corresponding row vectors also satisfy the asymptotic condition for the respective rows 2,3 and 4 in the $\mathrm{RH}$ problem for $M$. This will be done in the next proposition.

Proposition 8.14. The RH problem for $M$ has a unique solution that is constructed in the form

$$
M=\left(\begin{array}{cccc}
M_{11} & M_{12} & M_{13} & M_{14} \\
M_{11} F_{21} & M_{12} F_{22} & M_{13} F_{23} & M_{14} F_{24} \\
M_{11} F_{31} & M_{12} F_{32} & M_{13} F_{33} & M_{14} F_{34} \\
M_{11} F_{41} & M_{12} F_{42} & M_{13} F_{43} & M_{14} F_{44}
\end{array}\right)
$$

Proof. Let us do this for the second row. The asymptotic condition we get from (8.1) for the second row gives us as $z \rightarrow \infty$ in the first quadrant

$$
\begin{aligned}
& M_{11}(z) F_{21}(z)=\mathcal{O}\left(z^{-1}\right) \\
& M_{12}(z) F_{22}(z)=\frac{i}{\sqrt{3}} z^{1 / 3}+\mathcal{O}\left(z^{-2 / 3}\right) \\
& M_{13}(z) F_{23}(z)=-\frac{i}{\sqrt{3}} \omega z^{1 / 3}+\mathcal{O}\left(z^{-2 / 3}\right) \\
& M_{14}(z) F_{24}(z)=-\frac{i}{\sqrt{3}} \omega^{2} z^{1 / 3}+\mathcal{O}\left(z^{-2 / 3}\right)
\end{aligned}
$$

The first condition (8.37) is satisfied if and only if $F_{2}\left(\infty_{1}\right)=0$. The other conditions determine the behavior of $F_{2}$ near $\infty_{2}$.

There is an expansion

$$
F_{2}(w)=f_{3} w^{-3}+f_{2} w^{-2}+f_{1} w^{-1}+\mathcal{O}(1)
$$

where $w$ is the local coordinate near $\infty_{2}$ which we choose to be equal to $z^{-1 / 3}$ in the first quadrant of the second sheet. Its behavior in other quadrants and on other sheets is determined by analytic continuation. Thus

$$
F_{22}(z)=f_{3} z+f_{2} z^{2 / 3}+f_{1} z^{1 / 3}+\mathcal{O}(1)
$$

as $z \rightarrow \infty$ in the first quadrant. We also have

$$
M_{12}(z)=v_{2}(z)=m_{3} z^{-2 / 3}+m_{2} z^{-1}+m_{1} z^{-4 / 3}+\mathcal{O}\left(z^{-5 / 3}\right)
$$

as $z \rightarrow \infty$ in the first quadrant with a nonzero first coefficient

$$
m_{3} \neq 0 \text {. }
$$

Then inserting (8.41) and (8.42) into the condition (8.38) we obtain a linear system of equations for the unknowns $f_{3}, f_{2}, f_{1}$ that has a unique solution. These three conditions together with the fact that $F_{2}\left(\infty_{1}\right)=0$ determines $F_{2}$ uniquely.

Now it requires an independent check that the conditions (8.39) and (8.40) are satisfied as well with the same function $F_{2}$, and also the analogous conditions that come from the asymptotic condition in the other quadrants. This then completes the construction of the second row of $M$. 
The functions $F_{3}$ and $F_{4}$ are found in a similar way and they are used to construct the remaining rows of $M$.

From the construction it follows that the entries of $M$ and $M^{-1}$ are uniformly bounded in $n$ away from the branch points and infinity. More precisely, we have the following proposition.

Proposition 8.15. We have that

(a) the first columns of $M$ and $M^{-t}$ are bounded away from the branch points $a_{1}, b_{1}, \ldots, a_{N}, b_{N}$, with a bound that is uniform in $n$.

(b) the second columns of $M$ and $M^{-t}$ are bounded away from the branch points $a_{1}, b_{1}, \ldots, a_{N}, b_{N}, \pm i c_{2}$ and $\infty$, with a bound that is uniform in $n$.

(c) the third columns of $M$ and $M^{-t}$ are bounded away from the branch points $\pm i c_{2}, \pm c_{3}$ and $\infty$, with a bound that is uniform in $n$.

(d) the first columns of $M$ and $M^{-t}$ are bounded away from the branch points $\pm c_{3}$ and $\infty$, with a bound that is uniform in $n$.

Proof. We will only proof part (a) as the others follow by similar arguments.

Let us start with the first column of $M$. From the structure of the RH problem we see that the entries of $M$ in the first column are analytic in $\mathbb{C} \backslash\left[a_{1}, b_{n}\right]$, bounded near $\infty$ and have an analytic continuation across each interval $\left(a_{k}, b_{k}\right)$ and $\left(b_{k}, a_{k+1}\right)$. Hence the entries are bounded if we stay away from $\left\{a_{1}, b_{1}, \ldots, a_{N}, b_{N}\right\}$. Moreover, $M$ depends continuously on the parameters $n \alpha_{k}$ modulo integers. By compactness of the parameter space, it then follows that we can choose the bound for the entries such that they hold uniformly in $n$.

As for the first column of $M^{-t}$, we note that $M^{-t}$ satisfies a $\mathrm{RH}$ problem that has the same structure as the $\mathrm{RH}$ problem for $M$. Then the statement follows from the same arguments. Alternatively, the statement for $M^{-t}$ follows from the identity

$$
M^{-t}=\left(\begin{array}{cccc}
1 & 0 & 0 & 0 \\
0 & 0 & 0 & -1 \\
0 & 0 & -1 & 0 \\
0 & -1 & 0 & 0
\end{array}\right) \widetilde{M}
$$

where $\widetilde{M}$ is the solution of the $\mathrm{RH}$ problem (8.1) but with parameters $-\alpha_{k}$ instead of $\alpha_{k}$ for $k=1, \ldots, N$. The identity (8.43) follows from the fact that both sides solve the same, uniquely solvable, $\mathrm{RH}$ problem.

The following corollary will be used in the next chapter.

Corollary 8.16. Let $K \subset S\left(\mu_{1}\right) \backslash\left\{a_{1}, b_{1}, \ldots, a_{N}, b_{N}\right\}$ be compact. Then for every $x \in K$ we have

$$
M_{+}^{-1}(y) M_{+}(x)=\left(\begin{array}{cc}
I_{2}+\mathcal{O}(x-y) & * \\
* & *
\end{array}\right) \quad \text { as } y \rightarrow x,
$$

uniformly in $n$. The $*$ entries denote unimportant $2 \times 2$ blocks.

Proof. By Proposition 8.15 the first two columns in $M_{+}(x)-M_{+}(y)$ are uniformly bounded and since they are analytic it follows that they are of order $\mathcal{O}(x-y)$ as $y \rightarrow x$ uniformly in $n$. Also the first two rows of $M_{+}^{-1}(y)$ are uniformly bounded by Proposition 8.15 , Then the corollary follows, since

$$
M_{+}^{-1}(y) M_{+}(x)=I+M_{+}^{-1}(y)\left(M_{+}(x)-M_{-}(y)\right) .
$$




\section{Local Parametrices and Final Transformation}

\subsection{Local parametrices}

The global parametrix $M$ will not be a good approximation to $S$ near the branch points. Around the branch points we construct a local parametrix in a fairly standard way with the use of Airy functions. We will not give full details about the construct here but only give the relevant formulas with some comments.

\subsubsection{Statement of local RH problems. Let}

$$
B P=\left\{a_{k}, b_{k} \mid k=1, \ldots, n\right\} \cup\left(\left\{ \pm i c_{2}, \pm c_{3}\right\} \backslash\{0\}\right) .
$$

be the set of branch points. Note that $\pm i c_{2}$ an $\pm c_{3}$ are only branch points if they are non-zero.

There is a possibility that $\pm c_{3}$ coincides with one of the end points $a_{k}, b_{k}$ of the support of $\mu_{1}$. This is a case that could be handled just as well but the formulas are slightly different and we prefer not to give full detail in this case. Thus we assume

$$
\pm c_{3} \notin\left\{a_{k}, b_{k} \mid k=1, \ldots, n\right\} .
$$

We take a sufficiently small disk $D_{p}$ around each of the branch points $p \in B P$. The disks are mutually disjoint. We also make sure that the disks $D_{a_{k}}$ and $D_{b_{k}}$ are small enough such that they do not intersect with the lips of the global lens around $S\left(\mu_{3}\right)$, and similarly the disks $D_{ \pm c_{3}}$ do not intersect with the lips of the lenses around $S\left(\mu_{1}\right)$. Also the disks around $\pm i c_{2}$ are small enough so that they do not intersect with the lenses around $S\left(\mu_{1}\right)$ and $S\left(\mu_{3}\right)$.

We use

$$
D=\bigcup_{p \in B P} D_{p}
$$

to denote the union of the disks. Then we would like to find a parametrix $P$ : $\bar{D} \backslash \Sigma_{S} \rightarrow \mathbb{C}^{4 \times 4}$ that has the same jumps as $S$ on the parts of $\Sigma_{S}$ in $D$. There is a very minor complication here in case that $a_{k}$ or $b_{k}$ belongs to $\mathbb{R} \backslash S\left(\mu_{3}\right)=\left(-c_{3}, c_{3}\right)$. Then the jump matrix $J_{S}$ for $S$ has in its right lower block $\left(J_{S}\right)_{3}$ an off-diagonal entry that is exponentially small in a full neighborhood of $a_{k}$ or $b_{k}$, see the second formula in (7.25). Being exponentially small this entry will play no role in what follows. However, for the construction of the local parametrix it is more convenient to set this entry equal to 0 . This is also what we did when defining the part $\left(J_{M}\right)_{3}$ in the jump matrix for $M$, see (8.4). Thus we use $\left(J_{M}\right)_{3}$ for the jump matrix $\left(J_{P}\right)_{3}$ on the real line in $D_{a_{k}}$ and $D_{b_{k}}$, see (9.6) below.

A similar thing happens in case $\pm c_{3} \in \mathbb{R} \backslash S\left(\mu_{1}\right)$. Then an exponentially small entry is in $\left(J_{S}\right)_{1}$, see the second formula in (17.23) that we set equal to zero in the jump matrix for $P$. 
Thus in $D_{p}$ where $p$ is one of $a_{k}, b_{k}$, for $k=1, \ldots, N$, we take

$$
J_{P}=\left(\begin{array}{cc}
\left(J_{P}\right)_{1} & 0 \\
0 & \left(J_{P}\right)_{3}
\end{array}\right)
$$

with

$$
\begin{array}{ll}
\left(J_{P}\right)_{1}=\left(J_{S}\right)_{1} & \text { on } \Sigma_{S} \cap D_{p}, \\
\left(J_{P}\right)_{3}=\left(J_{M}\right)_{3} & \text { on } \mathbb{R} \cap D_{p}, \\
\left(J_{P}\right)_{3}=I_{2} & \text { on }\left(\Sigma_{S} \backslash \mathbb{R}\right) \cap D_{p} .
\end{array}
$$

If $c_{2}>0$ then in $D_{i c_{2}}$ and $D_{-i c_{2}}$ we put

$$
J_{P}=\left(\begin{array}{ccc}
1 & 0 & 0 \\
0 & \left(J_{P}\right)_{2} & 0 \\
0 & 0 & 1
\end{array}\right)
$$

with

$$
\left(J_{P}\right)_{2}=\left(J_{S}\right)_{2} \quad \text { on } \Sigma_{S} \cap D_{p} .
$$

If $c_{3}>0$ then in $D_{c_{3}}$ and $D_{-c_{3}}$, we have again the block structure (9.2), but now we put

$$
\begin{array}{ll}
\left(J_{P}\right)_{1}=\left(J_{M}\right)_{1} & \text { on } \mathbb{R} \cap\left(D_{c_{3}} \cup D_{-c_{3}}\right), \\
\left(J_{P}\right)_{1}=I_{2} & \text { on }\left(\Sigma_{S} \backslash \mathbb{R}\right) \cap\left(D_{c_{3}} \cup D_{-c_{3}}\right), \\
\left(J_{P}\right)_{3}=\left(J_{S}\right)_{3} & \text { on } \Sigma_{S} \cap\left(D_{c_{3}} \cup D_{-c_{3}}\right),
\end{array}
$$

With this definition of $J_{P}$ we look for a parametrix

$$
P: \bar{D} \backslash \Sigma_{S} \rightarrow \mathbb{C}^{4 \times 4}
$$

that satisfies the following local $\mathrm{RH}$ problem.

$$
\left\{\begin{array}{l}
P \text { is analytic in } D \backslash \Sigma_{S} \text { and continuous in } \bar{D} \backslash \Sigma_{S}, \\
P_{+}=P_{-} J_{P}, \quad \text { on } \Sigma_{S} \cap D, \\
P=\left(I+\mathcal{O}\left(n^{-1}\right)\right) M \quad \text { as } n \rightarrow \infty, \text { uniformly on } \partial D \backslash \Sigma_{S} .
\end{array}\right.
$$

9.1.2. Airy functions. Around each branch point the construction of $P$ is essentially a $2 \times 2$ problem, that can be solved in a standard way using Airy functions, see 33 for the $2 \times 2$ case and 20, 45] for larger size RH problems.

The $\mathrm{RH}$ problem for Airy functions is the following. It will be stated in terms of an auxiliary $\zeta$ variable.

$$
\left\{\begin{array}{l}
A: \mathbb{C} \backslash \Sigma_{A} \rightarrow \mathbb{C}^{2 \times 2} \text { is analytic, } \\
A_{+}=A_{-} J_{A}, \quad \text { on } \Sigma_{A}, \\
A(\zeta)=\left(\begin{array}{cc}
\zeta^{-1 / 4} & 0 \\
0 & \zeta^{1 / 4}
\end{array}\right) \frac{1}{\sqrt{2}}\left(\begin{array}{cc}
1 & i \\
i & 1
\end{array}\right)\left(I+\mathcal{O}\left(\zeta^{-3 / 2}\right)\right) \\
\quad \times\left(\begin{array}{cc}
e^{-\frac{2}{3} \zeta^{3 / 2}} & 0 \\
0 & e^{\frac{2}{3} \zeta^{3 / 2}}
\end{array}\right) \text { as } \zeta \rightarrow \infty
\end{array}\right.
$$

where the contour $\Sigma_{A}$ and the jump matrices $J_{A}$ are shown in Figure 9.1.

We have stated the $\mathrm{RH}$ problem in such a way that $\operatorname{det} A(\zeta) \rightarrow 1$ as $\zeta \rightarrow \infty$, which implies that also the solution has constant determinant 1 . This explains 


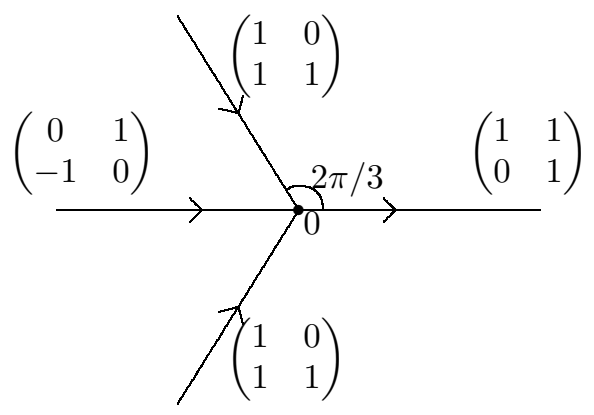

Figure 9.1. Contour $\Sigma_{A}$ and jump matrices $J_{A}$ in the RH problem for $A$.

the factors $\sqrt{2 \pi}$ and $\pm i$ that appear in the solution (9.9) below. Define the three functions

$$
y_{0}(\zeta)=\operatorname{Ai}(\zeta), \quad y_{1}(\zeta)=\omega \operatorname{Ai}(\omega \zeta), \quad y_{2}(\zeta)=\omega^{2} \operatorname{Ai}\left(\omega^{2} \zeta\right)
$$

where Ai denotes the usual Airy function. That is, $\mathrm{Ai}$ is the unique solution of the Airy differential equation $y^{\prime \prime}(\zeta)=\zeta y(\zeta)$ with asymptotic behavior

$$
\operatorname{Ai}(\zeta)=\frac{1}{2 \sqrt{\pi} \zeta^{1 / 4}} e^{-\frac{2}{3} \zeta^{3 / 2}}\left(1+\mathcal{O}\left(\zeta^{-3 / 2}\right)\right)
$$

as $\zeta \rightarrow \infty$ with $-\pi+\varepsilon<\arg \zeta<\pi-\varepsilon$ for any $\varepsilon>0$. Also $y_{1}$ and $y_{2}$ are solutions of the Airy equation and the relation

$$
y_{0}+y_{1}+y_{2}=0
$$

is satisfied.

Then the solution of the $\mathrm{RH}$ problem (9.8) is given by

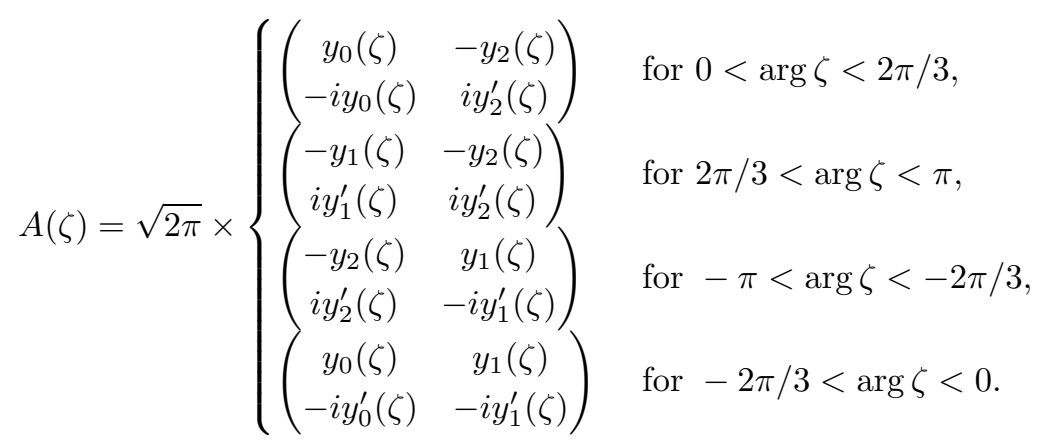

The solution $A$ of the Airy RH problem is the main building block for the local parametrix $P$.

9.1.3. Parametrix $P$ in $D_{b_{k}}$. In the neighborhood $D_{b_{k}}$ of a right-end point $b_{k}$ of the support of $\mu_{1}$ the local parametrix $P$ takes the form

$$
P(z)=M(z)\left(\begin{array}{cc}
P_{1}(z) & 0 \\
0 & I_{2}
\end{array}\right), \quad z \in D_{b_{k}} \backslash \Sigma_{S} .
$$


To describe the $2 \times 2$ block $P_{1}(z)$ we use the solution $A(\zeta)$ of the RH problem (9.8), the functions $\lambda_{1}$ and $\lambda_{2}$ that come from (4.35) (and that also appear in the jump matrix $\left(J_{P}\right)_{1}=\left(J_{S}\right)_{1}$, see (7.23) $)$, and a function

$$
f(z)= \begin{cases}\left(\frac{3}{4}\left(\lambda_{1}(z)-\lambda_{2}(z)\right) \pm \frac{3}{2} \pi i \alpha_{k}\right)^{2 / 3} & \text { if } b_{k}>0, \\ \left(\frac{3}{4}\left(\lambda_{1}(z)-\lambda_{2}(z)\right) \pm \frac{3}{2} \pi i \alpha_{k} \mp \frac{1}{2} \pi i\right)^{2 / 3} & \text { if } b_{k}<0,\end{cases}
$$

for $\pm \operatorname{Im} z>0, z \in D_{b_{k}} \backslash \mathbb{R}$.

It turns out that $f$ has an analytic extension to $D_{b_{k}}$ which maps $b_{k}$ to 0 (to check this one uses the jump properties (4.37)-(4.38) for $\lambda_{1}$ and $\lambda_{2}$, among other things). Shrinking $D_{b_{k}}$ if necessary, one has that $\zeta=f(z)$ is a conformal map from $D_{b_{k}}$ to a convex neighborhood of $\zeta=0$ with $f\left(b_{k}\right)=0, f^{\prime}\left(b_{k}\right)>0$ and which is real for real $z$. We adjust the lens around $\left(a_{k}, b_{k}\right)$ in such a way that the lips of the lens within $D_{b_{k}}$ are mapped by $f$ into the rays $\arg \zeta=2 \pi / 3$.

Then $P_{1}$ is given by

$$
\begin{aligned}
& P_{1}(z)=\left(\begin{array}{cc}
e^{ \pm n \pi i \alpha_{k}} & 0 \\
0 & e^{\mp n \pi i \alpha_{k}}
\end{array}\right) \frac{1}{\sqrt{2}}\left(\begin{array}{cc}
1 & -i \\
-i & 1
\end{array}\right)\left(\begin{array}{cc}
n^{1 / 6} f(z)^{1 / 4} & 0 \\
0 & n^{-1 / 6} f(z)^{-1 / 4}
\end{array}\right) \\
& \times A\left(n^{2 / 3} f(z)\right)\left(\begin{array}{cc}
e^{\frac{1}{2} n\left(\lambda_{1}(z)-\lambda_{2}(z)\right)} & 0 \\
0 & e^{-\frac{1}{2} n\left(\lambda_{1}(z)-\lambda_{2}(z)\right)}
\end{array}\right), \\
& \text { for } z \in D_{b_{k}} \backslash \Sigma_{S}, \pm \operatorname{Im} z>0,
\end{aligned}
$$

with the principal branches of the fourth roots in $f(z)^{ \pm 1 / 4}$.

9.1.4. Parametrix $P$ in $D_{a_{k}}$. The construction of $P$ in a neighborhood $D_{a_{k}}$ of a left endpoint $a_{k}$ of $S\left(\mu_{1}\right)$ is similar. Here we have a map

$$
f(z)= \begin{cases}\left(\frac{3}{4}\left(\lambda_{1}(z)-\lambda_{2}(z)\right) \pm \frac{3}{2} \pi i \alpha_{k-1}\right)^{2 / 3} & \text { if } a_{k}>0 \\ \left(\frac{3}{4}\left(\lambda_{1}(z)-\lambda_{2}(z)\right) \pm \frac{3}{2} \pi i \alpha_{k-1} \mp \frac{1}{2} \pi i\right)^{2 / 3} & \text { if } a_{k}<0\end{cases}
$$

for $\pm \operatorname{Im} z>0, z \in D_{a_{k}} \backslash \mathbb{R}$, with $f\left(a_{k}\right)=0, f^{\prime}\left(a_{k}\right)<0$. If necessary, we shrink the disk $D_{a_{k}}$ and adjust the lips of the lens around $\left(a_{k}, b_{k}\right)$ such that $\zeta=f(z)$ is a conformal map in $D_{a_{k}}$ onto a convex neighborhood of $\zeta=0$ that maps $\Sigma_{S} \cap D_{a_{k}}$ into $\Sigma_{A}$.

Then the local parametrix takes the form

$$
P(z)=M(z)\left(\begin{array}{cc}
P_{1}(z) & 0 \\
0 & I_{2}
\end{array}\right), \quad z \in D_{a_{k}} \backslash \Sigma_{S},
$$

with

$$
\begin{aligned}
& P_{1}(z)=\left(\begin{array}{cc}
e^{ \pm n \pi i \alpha_{k-1}} & 0 \\
0 & e^{\mp n \pi i \alpha_{k-1}}
\end{array}\right) \frac{1}{\sqrt{2}}\left(\begin{array}{cc}
1 & -i \\
i & -1
\end{array}\right)\left(\begin{array}{cc}
n^{1 / 6} f(z)^{1 / 4} & 0 \\
0 & n^{-1 / 6} f(z)^{-1 / 4}
\end{array}\right) \\
& \times A\left(n^{2 / 3} f(z)\right)\left(\begin{array}{cc}
1 & 0 \\
0 & -1
\end{array}\right)\left(\begin{array}{cc}
e^{\frac{1}{2} n\left(\lambda_{1}(z)-\lambda_{2}(z)\right)} & 0 \\
0 & e^{-\frac{1}{2} n\left(\lambda_{1}(z)-\lambda_{2}(z)\right)}
\end{array}\right) \\
& \text { for } z \in D_{a_{k}} \backslash \Sigma_{S}, \pm \operatorname{Im} z>0 \text {. }
\end{aligned}
$$

The fourth roots $f(z)^{ \pm 1 / 4}$ are taken to be analytic in $D_{a_{k}} \backslash\left[a_{k}, b_{k}\right]$ and positive for real $z<a_{k}, z \in D_{a_{k}}$. 
9.1.5. Parametrix $P$ in $D_{ \pm i c_{2}}$. This case is only relevant if $c_{2}>0$ and so we assume $c_{2}>0$. The parametrix in $D_{ \pm i c_{2}}$ takes the form

$$
P(z)=M(z)\left(\begin{array}{ccc}
1 & 0 & 0 \\
0 & P_{2}(z) & 0 \\
0 & 0 & 1
\end{array}\right)
$$

where $P_{2}(z)$ is a $2 \times 2$ matrix valued function in $D_{ \pm i c_{2}} \backslash \Sigma_{2}$.

In $D_{i c_{2}}$ it is constructed with the function

$$
f(z)=\left(\frac{3}{4}\left(\lambda_{2}(z)-\lambda_{3}(z)\right) \mp \frac{1}{2} \pi i\right)^{3 / 2} \quad \text { for } z \in D_{i c_{2}} \backslash i \mathbb{R}, \pm \operatorname{Re} z>0,
$$

which (with an appropriate understanding of the $3 / 2$-power) has an extension to a conformal map on $D_{i c_{2}}$ with $f\left(i c_{2}\right)=0$ and $f^{\prime}\left(i c_{2}\right) \in i \mathbb{R}^{+}$. Then $f(z)>0$ for $z=i y \in D_{i c_{2}} \cap i \mathbb{R}, y<c_{2}$. We take $P_{2}$ in $D_{i c_{2}}$ as

$$
\begin{aligned}
& P_{2}(z)=(-1)^{n} \frac{1}{\sqrt{2}}\left(\begin{array}{cc}
-i & 1 \\
-1 & i
\end{array}\right)\left(\begin{array}{cc}
n^{1 / 6} f(z)^{1 / 4} & 0 \\
0 & n^{-1 / 6} f(z)^{-1 / 4}
\end{array}\right) \\
& \times A\left(n^{2 / 3} f(z)\right)\left(\begin{array}{cc}
0 & -1 \\
1 & 0
\end{array}\right)\left(\begin{array}{cc}
e^{-\frac{1}{2} n\left(\lambda_{2}(z)-\lambda_{3}(z)\right)} & 0 \\
0 & e^{\frac{1}{2} n\left(\lambda_{2}(z)-\lambda_{3}(z)\right)}
\end{array}\right) \\
&
\end{aligned}
$$

The fourth roots $f(z)^{ \pm 1 / 4}$ are positive for $z=i y \in D_{i c_{2}} \cap i \mathbb{R}, y<c_{2}$.

The construction of $P$ in $D_{-i c_{2}}$ is very similar. By symmetry we can also obtain it from $P$ in $D_{i c_{2}}$ by means of the formula

$$
P(z)=\left(\begin{array}{cccc}
1 & 0 & 0 & 0 \\
0 & 1 & 0 & 0 \\
0 & 0 & -1 & 0 \\
0 & 0 & 0 & 1
\end{array}\right) P(-z)\left(\begin{array}{cccc}
1 & 0 & 0 & 0 \\
0 & 1 & 0 & 0 \\
0 & 0 & -1 & 0 \\
0 & 0 & 0 & 1
\end{array}\right)
$$

for $z \in D_{-i c_{2}} \cap \Sigma_{S}$.

9.1.6. Parametrix $P$ in $D_{ \pm c_{3}}$. This case is only relevant if $c_{3}>0$ and so we assume $c_{3}>0$. The parametrix $P$ in $D_{ \pm c_{3}}$ takes the form

$$
P(z)=M(z)\left(\begin{array}{cc}
I_{2} & 0 \\
0 & P_{3}(z)
\end{array}\right)
$$

where a $2 \times 2$ block $P_{3}(z)$.

It is constructed with the function

$$
f(z)=\left(\frac{3}{4}\left(\lambda_{3}(z)-\lambda_{4}(z)\right) \pm \frac{1}{4} \pi i\right)^{2 / 3} \quad \text { for } \pm \operatorname{Im} z>0, z \in D_{-c_{3}} \backslash \mathbb{R} .
$$

Then $f$ has an analytic extension to $D_{-c_{3}}$ which maps $\pm c_{3}$ to 0 . Shrinking $D_{ \pm c_{3}}$ if necessary, one has that $\zeta=f(z)$ is a conformal map from $D_{-c_{3}}$ to a neighborhood of $\zeta=0$ with $f\left(-c_{3}\right)=0$ and $f^{\prime}\left(-c_{3}\right)>0$. We adjust the lens around $\left(-\infty,-c_{3}\right)$ in such a way that the lips of the lens within $D_{-c_{3}}$ are mapped into the rays $\arg \zeta=2 \pi / 3$. Then $P_{3}$ is given by

$$
P_{3}(z)=(-1)^{n} \frac{1}{\sqrt{2}}\left(\begin{array}{cc}
1 & -i \\
-i & 1
\end{array}\right)\left(\begin{array}{cc}
n^{1 / 6} f(z)^{1 / 4} & 0 \\
0 & n^{-1 / 6} f(z)^{-1 / 4}
\end{array}\right)
$$




$$
\begin{aligned}
\times A\left(n^{2 / 3} f(z)\right)\left(\begin{array}{cc}
e^{\frac{1}{2} n\left(\lambda_{3}(z)-\lambda_{4}(z)\right)} & 0 \\
0 & e^{-\frac{1}{2} n\left(\lambda_{3}(z)-\lambda_{4}(z)\right)}
\end{array}\right) & , \\
& \text { for } z \in D_{-c_{3}} \backslash \Sigma_{S} .
\end{aligned}
$$

For the construction in $D_{c_{3}}$ we can use the conformal map $f$ with the same definition as in (9.20), but now considered in the neighborhood of $c_{3}$. Then we have $f\left(c_{3}\right)=0$ and $f^{\prime}\left(c_{3}\right)<0$, and after adjusting of the lenses we then define $P_{3}$ as

$$
\begin{aligned}
& P_{3}(z)=(-1)^{n} \frac{1}{\sqrt{2}}\left(\begin{array}{cc}
1 & -i \\
i & -1
\end{array}\right)\left(\begin{array}{cc}
n^{1 / 6} f(z)^{1 / 4} & 0 \\
0 & n^{-1 / 6} f(z)^{-1 / 4}
\end{array}\right) \\
& \times A\left(n^{2 / 3} f(z)\right)\left(\begin{array}{cc}
1 & 0 \\
0 & -1
\end{array}\right)\left(\begin{array}{cc}
e^{\frac{1}{2} n\left(\lambda_{3}(z)-\lambda_{4}(z)\right)} & 0 \\
0 & e^{-\frac{1}{2} n\left(\lambda_{3}(z)-\lambda_{4}(z)\right)}
\end{array}\right), \\
& \text { for } z \in D_{c_{3}} \backslash \Sigma_{S} .
\end{aligned}
$$

\subsection{Final transformation}

Having the global parametrix $M$ and the local parametrix $P$ we are ready for the fifth and also final transformation.

Definition 9.1. We define the $4 \times 4$ matrix valued function $R$ by

$$
R(z)= \begin{cases}S(z) P(z)^{-1} & \text { in the disks around each of the branch points } \\ S(z) M(z)^{-1} & \text { outside the disks. }\end{cases}
$$

Then $R$ is defined and analytic outside the union of $\Sigma_{S}$ and the boundaries of the disks around the branch points. However, since the jumps of $S$ and $M$ agree on $S\left(\mu_{1}\right) \cap S\left(\mu_{3}\right)$ and on $S\left(\sigma_{2}-\mu_{2}\right)$, the matrix $R$ has analytic continuation across the parts of these supports that are outside the disks. We also have that the jumps of $S$ and $P$ agree inside the disks $D_{ \pm i c_{2}}$ and so $R$ has analytic continuation in the interior of these two disks. In the disk $D_{a_{k}}, D_{b_{k}}$ and $D_{ \pm c_{3}}$ the jumps of $S$ and $P$ may not be exactly the same. They could differ by an exponentially small entry on the real line inside these disks. The jumps on the lips of the lenses are the same inside the disks, and so $R$ has an analytic continuation across these lenses, but $R$ could have an exponentially small jump on the real line inside the disks.

The result is that $R$ has an analytic continuation to $\mathbb{C} \backslash \Sigma_{R}$ for a certain contour $\Sigma_{R}$ and that $R$ satisfies the following $\mathrm{RH}$ problem.

$$
\left\{\begin{array}{l}
R \text { is analytic in } \mathbb{C} \backslash \Sigma_{R}, \\
R_{+}=R_{-} J_{R}, \quad \text { on } \Sigma_{R}, \\
R(z)=I+\mathcal{O}\left(z^{-1}\right) \quad \text { as } z \rightarrow \infty .
\end{array}\right.
$$

where

$$
J_{R}(z)= \begin{cases}M(z) P(z)^{-1}, & z \in \bigcup_{p \in B P} \partial D_{p}, \\ P_{-}(z) J_{S}(z) P_{+}(z)^{-1}, & z \in \Sigma_{R} \cap \bigcup_{p \in B P} D_{p} \\ M_{-}(z) J_{S}(z) M_{+}(z)^{-1}, & z \in \Sigma_{R} \backslash \overline{\bigcup_{p \in B P} D_{p}}\end{cases}
$$

All of the jump matrices are close to the identity matrix as $n \rightarrow \infty$. Indeed because of the matching condition in (9.7) we have

$$
J_{R}(z)=I_{4}+\mathcal{O}\left(n^{-1}\right) \quad \text { as } n \rightarrow \infty
$$


uniformly for $z \in \bigcup_{p \in B P} \partial D_{p}$. The other jumps are exponentially close to the identity matrix as $n \rightarrow \infty$ with a bound that improves as $z \rightarrow \infty$. Indeed we have

$$
J_{R}(z)=I_{4}+\mathcal{O}(\exp (-c n(|z|+1)) \quad \text { as } n \rightarrow \infty
$$

uniformly for $z \in \Sigma_{R} \backslash\left(\bigcup_{p \in B P} \partial D_{p}\right)$. This follows as in [45, Section 8.4].

Observe that from the definition (9.23) and the asymptotic behaviors of $S$ and $M$ as given in (7.20) and (8.1) we first find that $R(z)=I_{4}+\mathcal{O}\left(z^{-1 / 3}\right)$ as $z \rightarrow \infty$. However $J_{R}-I_{4}$ is also exponentially decaying as $z \rightarrow \infty$ with $n$ fixed, and therefore the better bound $R(z)=I_{4}+\mathcal{O}\left(z^{-1}\right)$ in (9.24) indeed holds.

From this we conclude as in 45 .

Proposition 9.2. There is a constant $C>0$ such that for every $n$,

$$
\left\|R(z)-I_{4}\right\| \leq \frac{C}{n(|z|+1)}
$$

uniformly for $z \in \mathbb{C} \backslash \Sigma_{R}$.

This concludes the steepest descent analysis of the RH problem for $Y$.

\subsection{Proof of Theorem 1.4}

We use the following consequence of Corollary 8.16 and Proposition 9.2

Lemma 9.3. For every $x \in S\left(\mu_{1}\right) \backslash \bigcup_{k=1}^{N}\left(D_{a_{k}} \cup D_{b_{k}}\right)$ we have

$$
S_{+}^{-1}(y) S_{+}(x)=\left(\begin{array}{cc}
I_{2}+\mathcal{O}(x-y) & * \\
* & *
\end{array}\right) \quad \text { as } y \rightarrow x,
$$

uniformly in $n$. The * entries denote unimportant $2 \times 2$ blocks.

Proof. By (9.23) and the fact that $x$ is outside the disks, we have

$$
S_{+}^{-1}(y) S_{+}(x)=M_{+}^{-1}(y) R_{+}^{-1}(y) R_{+}(x) M_{+}(x),
$$

if $y$ is close enough to $x$. Note that $R$ may not be analytic in a neighborhood of $x$. However, by deforming contours into the lower half plane we see that $R$ does have an analytic continuation to a neighborhood of $x$ where the same estimate of Proposition 9.2 is valid. Now write

$$
R_{+}^{-1}(y) R_{+}(x)=I+R_{+}^{-1}(y)\left(R_{+}(x)-R_{+}(y)\right) .
$$

Then by Cauchy's Theorem

$$
\begin{aligned}
R_{+}^{-1}(y) R_{+}(x) & =I+R_{+}^{-1}(y) \frac{1}{2 \pi i} \oint_{|z-x|=r} R(z)\left(\frac{1}{z-x}-\frac{1}{z-y}\right) d z \\
& =I+R_{+}^{-1}(y) \frac{(x-y)}{2 \pi i} \oint_{|z-x|=r} \frac{R(z)-I}{(z-x)(z-y)} d z,
\end{aligned}
$$

for some $r>0$. Combining this with Proposition 9.2 leads to

$$
R_{+}^{-1}(y) R_{+}(x)=I+\mathcal{O}\left(\frac{x-y}{n}\right) \quad \text { as } y \rightarrow x .
$$

Now inserting this in (9.26) and using Corollary 8.16 gives the statement. 
To prove Theorem 1.4 we follow the effect of the transformations $Y \mapsto X \mapsto$ $U \mapsto T \mapsto S \mapsto R$ on the kernel $K_{11}^{(n)}$ given by (1.37).

The transformation $Y \mapsto X$ as given by (5.17) then gives

$$
\begin{aligned}
& K_{11}^{(n)}(x, y)=\frac{1}{2 \pi i(x-y)}\left(0 \quad w_{0, n}(y) \quad w_{1, n}(y) \quad w_{2, n}(y)\right) \\
& \times\left(\begin{array}{cc}
1 & 0 \\
0 & D_{n} P_{n,+}^{-t}(y) e^{n \Theta_{+}(y)}
\end{array}\right) X_{+}^{-1}(y) X_{+}(x) \\
& \times\left(\begin{array}{cc}
1 & 0 \\
0 & e^{-n \Theta_{+}(x)} P_{n,+}^{t}(x) D_{n}^{-1}
\end{array}\right)\left(\begin{array}{l}
1 \\
0 \\
0 \\
0
\end{array}\right) .
\end{aligned}
$$

By (5.26) we then get

$$
K_{11}^{(n)}(x, y)=\frac{1}{2 \pi i(x-y)}\left(\begin{array}{llll}
0 & e^{-n\left(V(y)-\theta_{1}(y)\right)} & 0 & 0
\end{array}\right) X_{+}^{-1}(y) X_{+}(x)\left(\begin{array}{l}
1 \\
0 \\
0 \\
0
\end{array}\right) .
$$

Then by the transformation $X \mapsto U$ given in (6.1) we get

$$
\begin{aligned}
& K_{11}^{(n)}(x, y)=\frac{e^{n\left(g_{1,+}(x)+\ell_{1}\right)}}{2 \pi i(x-y)}\left(0 \quad e^{-n\left(V(y)-\theta_{1}(y)-g_{1,+}(y)+g_{2,+}(y)\right)} \quad 0\right. \\
& \times U_{+}^{-1}(y) U_{+}(x)\left(\begin{array}{l}
1 \\
0 \\
0 \\
0
\end{array}\right), \quad x, y \in \mathbb{R} \text {. }
\end{aligned}
$$

By (4.35) this leads to

$$
\begin{aligned}
& K_{11}^{(n)}(x, y)=\frac{e^{n\left(g_{1,+}(x)-g_{1,+}(y)\right)}}{2 \pi i(x-y)}\left(\begin{array}{lll}
0 & e^{n\left(\lambda_{2,+}(y)-\lambda_{1,+}(y)\right)} & 0
\end{array}\right. \\
& \times U_{+}^{-1}(y) U_{+}(x)\left(\begin{array}{l}
1 \\
0 \\
0 \\
0
\end{array}\right), \quad x, y \in \mathbb{R} \text {. }
\end{aligned}
$$

The transformation $U \mapsto T$ given by (7.6) -(7.8) only acts on the lower right $2 \times 2$ block, and does not affect the expression (9.30) for the correlation kernel. We get

$$
\begin{aligned}
& K_{11}^{(n)}(x, y)=\frac{e^{n\left(g_{1,+}(x)-g_{1,+}(y)\right)}}{2 \pi i(x-y)}\left(\begin{array}{llll}
0 & e^{n\left(\lambda_{2,+}(y)-\lambda_{1,+}(y)\right)} & 0 & 0
\end{array}\right) \\
& \times T_{+}^{-1}(y) T_{+}(x)\left(\begin{array}{l}
1 \\
0 \\
0 \\
0
\end{array}\right), \quad x, y \in \mathbb{R} \text {. }
\end{aligned}
$$


Next, by the transformation $T \mapsto S$ of (7.18) and (17.19) we find

$$
\begin{aligned}
& K_{11}^{(n)}(x, y)=\frac{e^{n\left(g_{1,+}(x)-g_{1,+}(y)\right)}}{2 \pi i(x-y)}\left(-\chi_{S\left(\mu_{1}\right)}(y) \quad e^{n\left(\lambda_{2,+}(y)-\lambda_{1,+}(y)\right)} \quad 0 \quad 0\right) \\
& \times S_{+}^{-1}(y) S_{+}(x)\left(\begin{array}{c}
1 \\
\chi_{S\left(\mu_{1}\right)}(x) e^{n\left(\lambda_{1,+}(x)-\lambda_{2,+}(x)\right)} \\
0 \\
0
\end{array}\right), \quad x, y \in \mathbb{R}
\end{aligned}
$$

where $\chi_{S\left(\mu_{1}\right)}$ denotes the characteristic function of the set $S\left(\mu_{1}\right)$.

Let $x \in S\left(\mu_{1}\right) \backslash\left\{a_{k}, b_{k} \mid k=1, \ldots, N\right\}$. The factor $e^{n\left(g_{1,+}(x)-g_{1,+}(y)\right)}$ disappears as $y \rightarrow x$. Then we use Lemma 9.3 and we get

$$
\begin{aligned}
K_{11}^{(n)}(x, x) & =\lim _{y \rightarrow x} \frac{-1+e^{n\left(\lambda_{2,+}(y)-\lambda_{1,+}(y)\right)} e^{n\left(\lambda_{1,+}(x)-\lambda_{2,+}(x)\right)}}{2 \pi i(x-y)}+\mathcal{O}(1) \\
& =\frac{n}{2 \pi i} \frac{d}{d x}\left(\lambda_{1,+}(x)-\lambda_{2,+}(x)\right)+\mathcal{O}(1)
\end{aligned}
$$

as $n \rightarrow \infty$.

Thus

$$
\begin{aligned}
\lim _{n \rightarrow \infty} \frac{1}{n} K_{11}^{(n)}(x, x) & =\frac{1}{2 \pi i} \frac{d}{d x}\left(\lambda_{1,+}(x)-\lambda_{2,+}(x)\right) \\
& =\frac{1}{2 \pi i}\left(F_{1,-}(x)-F_{1,+}(x)\right) \\
& =\frac{d \mu_{1}}{d x},
\end{aligned}
$$

where we used (4.35), (4.10), (4.13), and (4.14). This completes the proof of Theorem [1.4. 


\section{Bibliography}

[1] M. Adler, P. Ferrari, and P. van Moerbeke, Non-intersecting random walks in the neighborhood of a symmetric tacnode, preprint arXiv:1007.1163.

[2] M. Adler and P. van Moerbeke, The spectrum of coupled random matrices, Ann. of Math. (2) 149 (1999), 921-976. MR1709307 (2001e:82004)

[3] M. Adler and P. van Moerbeke, PDEs for the Gaussian ensemble with external source and the Pearcey distribution, Comm. Pure Appl. Math. 60 (2007), 1261-1292. MR2337504 (2009c:82021)

[4] A.I. Aptekarev, Multiple orthogonal polynomials, J. Comput. Appl. Math. 99 (1998), 423447. MR1662713 (99m:42036)

[5] A.I. Aptekarev, Strong asymptotics of multiple orthogonal polynomials for Nikishin systems, Mat. Sb. 190 (1999), 3-44 (Russian); Sbornik Math. 190 (1999), 631-669. MR.1702555 (2001a:42023)

[6] A.I. Aptekarev, V.A. Kalyagin, and E.B. Saff, Higher-order three-term recurrences and asymptotics of multiple orthogonal polynomials, Constr. Approx. 30 (2009), 175-223. MR2519660(2010h:42049)

[7] J. Baik, T. Kriecherbauer, K.T.-R. McLaughlin, and P.D. Miller, Discrete Orthogonal Polynomials. Asymptotics and Applications, Ann. Math. Studies 164, Princeton University Press, Princeton, NJ, 2007. MR2283089 (2011b:42081)

[8] F. Balogh and M. Bertola, Regularity of a vector potential problem and its spectral curve, J. Approx. Theory 161 (2009), 353-370. MR2558160 (2011a:42036)

[9] M. Bergère and B. Eynard, Mixed correlation function and spectral curve for the two-matrix model, J. Phys. A 39 (2006), 15091-15134. MR2277092(2007i:82032)

[10] M. Bertola, Biorthogonal polynomials for two-matrix models with semiclassical potentials, J. Approx. Theory 144 (2007), 162-212. MR2293386 (2009i:34202)

[11] M. Bertola, Two-matrix models and biorthogonal polynomials, Chapter 15 in: "The Oxford Handbook of Random Matrix Theory", (G. Akemann, J. Baik, and P. Di Francesco, eds.), Oxford University Press, 2011.

[12] M. Bertola and B. Eynard, The PDEs of biorthogonal polynomials arising in the two-matrix model, Math. Phys. Anal. Geom. 9 (2006), 23-52. MR2245260 (2007c:34135)

[13] M. Bertola, B. Eynard, and J. Harnad, Duality, biorthogonal polynomials and multi-matrix models, Comm. Math. Phys. 229 (2002), 73-120. MR1917675 (2003d:82045)

[14] M. Bertola, B. Eynard and J. Harnad, Differential systems for biorthogonal polynomials appearing in 2-matrix models and the associated Riemann-Hilbert problem, Comm. Math. Phys. 243 (2003), 193-240. MR2021905 (2004i:34237)

[15] M. Bertola, M. Gekhtman, and J. Szmigielski, The Cauchy two-matrix model, Comm. Math. Phys. 287 (2009), 983-1014. MR2486670(2010a:42090)

[16] M. Bertola and S.Y. Lee, First colonization of a spectral outpost in random matrix theory, Constr. Approx. 30 (2009), 225-263. MR2519661 (2010h:15056)

[17] P. Bleher, S. Delvaux, and A.B.J. Kuijlaars, Random matrix model with external source and a constrained vector equilibrium problem, Comm. Pure Appl. Math 64 (2011), 116-160.

[18] P. Bleher and A. Its, Semiclassical asymptotics of orthogonal polynomials, Riemann-Hilbert problem, and universality in the matrix model, Ann. of Math. (2)150 (1999), 185-266. MR.1715324 (2000k:42033)

[19] P.M. Bleher and A.R. Its, Double scaling limit in the random matrix model: the RiemannHilbert approach, Comm. Pure Appl. Math. 56 (2003), 433-516. MR.1949138 (2004e:82024)

[20] P.M. Bleher and A.B.J. Kuijlaars, Large $n$ limit of Gaussian random matrices with external source, part I, Comm. Math. Phys. 252 (2004), 43-76. MR2103904 (2005i:82031) 
[21] P. Bleher and A.B.J. Kuijlaars, Large $n$ limit of Gaussian random matrices with external source, part III: double scaling limit, Comm. Math. Phys. 270 (2007), 481-517. MR 2276453 (2008b:82032)

[22] P. Bleher and K. Liechty, Uniform asymptotics for discrete orthogonal polynomials with respect to varying exponential weights on a regular infinite lattice, Int. Math. Res. Notices 2010 (2010) article ID rnq081.

[23] A. Borodin, Biorthogonal ensembles, Nucl. Phys. B 536, (1999), no. 3, 704-732. MR.1663328 (99m:82022)

[24] A. Borodin and E. Rains, Eynard-Mehta theorem, Schur process, and their Pfaffian analogs, J. Stat. Phys. 121 (2005),291-317. MR2185331 (2006k:82039)

[25] E. Brézin and S. Hikami, Universal singularity at the closure of a gap in a random matrix theory, Phys. Rev. E 57 (1998), 4140-4149. MR1618958 (99i:82021)

[26] T. Claeys, The birth of a cut in unitary random matrix ensembles, Int. Math. Res. Notices 2008 (2008), article ID rnm166, 40 pages. MR2427458 (2010a:15078)

[27] T. Claeys and A.B.J. Kuijlaars, Universality of the double scaling limit in random matrix models, Comm. Pure Appl. Math. 59 (2006), 1573-1603. MR2254445 (2007g:82019)

[28] T. Claeys and M. Vanlessen, Universality of a double scaling limit near singular edge points in random matrix models, Comm. Math. Phys. 273 (2007), 499-532. MR2318316 (2009d:15056)

[29] E. Daems and A.B.J. Kuijlaars, A Christoffel-Darboux formula for multiple orthogonal polynomials, J. Approx. Theory 130, (2004), 190-202. MR2100703 (2005g:42059)

[30] J.M. Daul, V. Kazakov, and I.K. Kostov, Rational theories of $2 D$ gravity from the two-matrix model, Nucl. Phys. B 409 (1993), 311-338. MR1246736 (94j:81214)

[31] P. Deift, Orthogonal polynomials and random matrices: a Riemann-Hilbert approach, Courant Lecture Notes 3. New York University, 1999. MR.1677884 (2000g:47048)

[32] P. Deift, T. Kriecherbauer, and K.T.-R. McLaughlin, New results on the equilibrium measure for logarithmic potentials in the presence of an external field, J. Approx. Theory 95 (1998), 388-475. MR 1657691 (2000j:31003)

[33] P. Deift, T. Kriecherbauer, K.T.-R. McLaughlin, S. Venakides, and X. Zhou, Uniform asymptotics for polynomials orthogonal with respect to varying exponential weights and applications to universality questions in random matrix theory, Comm. Pure Appl. Math. 52 (1999), 1335-1425. MR:1702716 (2001g:42050)

[34] P. Deift, T. Kriecherbauer, K.T.-R. McLaughlin, S. Venakides, and X. Zhou, Strong asymptotics of orthogonal polynomials with respect to exponential weights, Comm. Pure Appl. Math. 52 (1999), 1491-1552. MR.1711036 (2001f:42037)

[35] P. Deift and X. Zhou, A steepest descent method for oscillatory Riemann-Hilbert problems. Asymptotics for the MKdV equation, Ann. of Math. (2) 137 (1993), 295-368. MR1207209 (94d:35143)

[36] Ch.-J. de la Vallée Poussin, Potentiel et problème généralise de Dirichlet, Math. Gaz. 22 (1938), 17-36.

[37] S. Delvaux, Average characteristic polynomials in the two-matrix model, J. Math. Phys. 52 (2011), 23pp. MR2791135

[38] S. Delvaux, A.B.J. Kuijlaars, and L. Zhang, Critical behavior of non-intersecting Brownian motions at a tacnode, Comm. Pure Appl. Math. 64 (2011), 1305-1383.

[39] P. Di Francesco, 2D topological and quantum gravities, matrix models and integrable differential systems, in: "The Painlevé property", (R. Conte ed.) CRM Ser. Math. Phys., Springer, New York, (1999), pp. 229-285. MR1713579(2000j:81239)

[40] P. Di Francesco, P. Ginsparg, J. Zinn-Justin, 2D gravity and random matrices, Phys. Rep. 254 (1995), 1-169. MR1320471 (96c:81191)

[41] M.R. Douglas, The two-matrix model, in: "Random surfaces and quantum gravity", NATO Adv. Sci. Inst. Ser. B Phys., 262, Plenum, New York, (1991), pp. 77-83. MR1214383

[42] P.D. Dragnev and E.B. Saff, Constrained energy problems with applications to orthogonal polynomials of a discrete variable, J. Anal. Math. 72 (1997), 223-259. MR.1482996 (99f:42048)

[43] M. Duits, D. Geudens and A.B.J. Kuijlaars, A vector equilibrium problem for the two-matrix model in the quartic/quadratic case, Nonlinearity 24 (2011), no. 3, 951-993. MR2772631

[44] M. Duits and A.B.J. Kuijlaars, An equilibrium problem for the limiting eigenvalue distribution of banded Toeplitz matrices, SIAM J. Matrix Anal. Appl. 30 (2008), 173-196. MR2399575 (2009b:15021) 
[45] M. Duits and A.B.J. Kuijlaars, Universality in the two matrix model: a Riemann-Hilbert steepest descent analysis, Comm. Pure Appl. Math. 62, (2009), 1076-1153. MR2531553 (2010f:82046)

[46] N.M. Ercolani and K.T.-R. McLaughlin, Asymptotics and integrable structures for biorthogonal polynomials associated to a random two-matrix model, Physica D 152-153 (2001), 232-268. MR 1837912 (2002g:81148)

[47] B. Eynard, Eigenvalue distribution of large random matrices, from one matrix to several coupled matrices, Nucl. Phys. B 506 (1997), 633-664. MR1488592 (99f:82033)

[48] B. Eynard, Large- $N$ expansion of the 2-matrix model, J. High Energy Phys. (2003), no. 1, 051, 38pp. MR1969872 (2004h:82045)

[49] B. Eynard, The 2-matrix model, biorthogonal polynomials, Riemann-Hilbert problem, and algebraic geometry, preprint arXiv:math-ph/0504034.

[50] B. Eynard and M. L. Mehta, Matrices coupled in a chain. I. Eigenvalue correlations, J. Phys. A 31 (1998), 4449-4456. MR.1628667 (99g:82028)

[51] B. Eynard and N. Orantin, Mixed correlation functions in the 2-matrix model, and the Bethe ansatz, J. High Energy Phys. (2005), no. 8, 028, 36p. MR2165818 (2006e:81209)

[52] B. Eynard and N. Orantin, Topological expansion of the 2-matrix model correlation functions: diagrammatic rules for a residue formula, J. High Energy Phys. (2005), no. 2, 034, 44pp. MR:2197034 (2007m:81185)

[53] H.M. Farkas and I. Kra, Riemann surfaces, Graduate Texts in Mathematics 71, SpringerVerlag, New York-Berlin, 1980. MR.583745 (82c:30067)

[54] J. Fay, Theta functions on Riemann surfaces, Springer-Verlag, Berlin, 1973. MR0335789 $(49: 569)$

[55] A.S. Fokas, A.R. Its, and A.V. Kitaev, The isomonodromy approach to matrix models in $2 D$ quantum gravity, Comm. Math. Phys. 147 (1992), 395-430. MR1174420 (93h:81115)

[56] A.A. Gonchar and E.A. Rakhmanov, The equilibrium problem for vector potentials (Russian) Uspekhi Mat. Nauk 40 (1985), 155-156. MR807734

[57] A. Guionnet, First order asymptotics of matrix integrals; a rigorous approach towards the understanding of matrix models, Comm. Math. Phys. 244 (2004), 527-569 MR2034487 (2005c:82043)

[58] J. Huisman, On the geometry of algebraic curves having many real components, Rev. Mat. Complut. 14 (2001), 83-92. MR.1851723 (2002f:14074)

[59] C. Itzykson and J. B. Zuber, The planar approximation II, J. Math. Phys. 21 (1980), 411-421. MR.562985 (81a:81068)

[60] K. Johansson, Non-colliding Brownian Motions and the extended tacnode process, preprint arXiv:1105.4027.

[61] A.A. Kapaev, Riemann-Hilbert problem for bi-orthogonal polynomials, J. Phys. A 36 (2003), 4629-4640. MR1985213 (2004m:34206)

[62] V.A. Kazakov, Ising model on a dynamical planar random lattice: exact solution, Phys. Lett. A 119, (1986), 140-144. MR871244 (88g:81092)

[63] A.B.J. Kuijlaars, Multiple orthogonal polynomial ensembles, in "Recent Trends in Orthogonal Polynomials and Approximation Theory" (J. Arvesú, F. Marcellán and A. MartínezFinkelshtein eds.), Contemporary Mathematics 507, 2010, pp. 155-176. MR2647568

[64] A.B.J. Kuijlaars, Multiple orthogonal polynomials in random matrix theory, in: "Proceedings of the International Congress of Mathematicians, Hyderabad 2010, Volume III", (R. Bhatia et al. eds.), World Scientific Publishing Co, 2010, pp. 1417-1432. MR2647568

[65] A.B.J. Kuijlaars and P.D. Dragnev, Equilibrium problems associated with fast decreasing polynomials, Proc. Amer. Math. Soc. 127 (1999), 1065-1074. MR1469419 (99f:31003)

[66] A.B.J. Kuijlaars, A. Martínez-Finkelshtein, and F. Wielonsky, Non-intersecting squared Bessel paths and multiple orthogonal polynomials for modified Bessel weights, Comm. Math. Phys. 286 (2009), 217-275. MR2470930 (2010h:60112)

[67] A.B.J. Kuijlaars and K.T.-R. McLaughlin, A Riemann-Hilbert problem for biorthogonal polynomials, J. Comput. Appl. Math. 178 (2005), 313-320. MR2127887 (2006a:15046)

[68] A.B.J. Kuijlaars and M.Y. Mo, The global parametrix in the Riemann-Hilbert steepest descent analysis for orthogonal polynomials, Comput. Methods Funct. Theory 11 (2011), 161-178.

[69] A.B.J. Kuijlaars and E. Rakhmanov, Zero distributions for discrete orthogonal polynomials, J. Comp. Appl. Math. 99 (1998), 255-274. MR.1662699 (2000c:42025a) 
[70] A.B.J. Kuijlaars and P. Román, Recurrence relations and vector equilibrium problems arising from a model of non-intersecting squared Bessel paths, J. Approx. Theory 162 (2010), 20482077.

[71] M.L. Mehta, A method of integration over matrix variables, Comm. Math. Phys. 79 (1981), 327-340. MR627056 (83c:81032)

[72] M.L. Mehta, Random Matrices, 3rd edition, Pure and Applied Mathematics Vol. 142. Elsevier/Academic Press, Amsterdam, 2004. MR2129906 (2006b:82001)

[73] M.L. Mehta and P. Shukla, Two coupled matrices: eigenvalue correlations and spacing functions, J. Phys. A 27 (1994), 7793-7803. MR.1312283(96b:82013)

[74] P. Miller, Applied Asymptotic Analysis, Amer. Math. Soc., Providence, RI, 2006. MR2238098 (2007e:34002)

[75] M.Y. Mo, The Riemann-Hilbert approach to double scaling limit of random matrix eigenvalues near the "birth of a cut" transition, Internat. Math. Res. Notices 2008 (2008), article ID rnn042, 51 pages. MR2436561 (2010c:60020)

[76] M.Y. Mo, Universality in the two matrix model with a monomial quartic and a general even polynomial potential, Comm. Math. Phys. 291 (2009), 863-894. MR2534795(2010h:82045)

[77] E.M. Nikishin and V.N. Sorokin, Rational Approximations and Orthogonality, Transl. Math. Monogr. 92, Amer. Math. Soc., Providence, R.I., 1991. MR.1130396 (92i:30037)

[78] E.A. Rakhmanov, Equilibrium measure and the distribution of zeros of the extremal polynomials of a discrete variable, Mat. Sb. 187 (1996), 109-124 (Russian); Sbornik Math. 187 (1996), 1213-1228. MR.1418343 (97g:26015)

[79] E.B. Saff and V. Totik, Logarithmic Potentials with External Field, Grundlehren der Mathematischen Wissenschaften 316, Springer-Verlag, Berlin, 1997. MR1485778 (99h:31001)

[80] M. Shcherbina, Double scaling limit for matrix models with nonanalytic potentials, J. Math. Phys. 49 (2008), 033501, 34 pp. MR2406798 (2009b:82042)

[81] V. Totik and J.L. Ullman, Local asymptotic distribution of zeros of orthogonal polynomials, Trans. Amer. Math. Soc. 341 (1994),881-894. MR.1150019 (94d:42031)

[82] C. Tracy and H. Widom, The Pearcey process, Comm. Math. Phys. 263 (2006), 381-400. MR2207649(2006m:82101)

[83] W. Van Assche, Multiple orthogonal polynomials, irrationality and transcendence, in: "Continued Fractions: from Analytic Number Theory to Constructive Approximation" (B. Berndt and F. Gesztesy, eds.), Contemp. Math. 236, Amer. Math. Soc., Providence RI, 1999, pp. 325-342. MR1665377 (2000k:42039)

[84] W. Van Assche, J.S. Geronimo, and A.B.J. Kuijlaars, Riemann-Hilbert problems for multiple orthogonal polynomials, in: "Special Functions 2000" (J. Bustoz et al., eds.), Kluwer, Dordrecht 2001, pp. 23-59. MR2006283 (2004e:30065)

[85] L. Zhang and P. Román, Asymptotic zero distribution of multiple orthogonal polynomials associated with Macdonald functions, J. Approx. Theory, 163 (2011), 143-162. 


\title{
Index
}

\author{
Symbols \\ $\left(J_{P}\right)_{k}, 90$ \\ $A(\zeta), 91$ \\ $A(z), 54$ \\ $A_{j}, 4577$ \\ $B P, 89$ \\ $B_{j}, 77$ \\ $C_{n}, 45$ \\ $D, 86$ \\ $D_{n}, 45$ \\ $D_{p}, 89$ \\ $F_{j}, 33$ \\ $F_{j k}, 86$ \\ $G(z), 53$ \\ $J_{A}, 90$ \\ $J_{P}, 90$ \\ $J_{R}, 94$ \\ $J_{X}$, 46 \\ $L, 53$ \\ $L_{1}, 68$ \\ $L_{2}, 61$ \\ $L_{3}, 63$ \\ $M(z), 75,87$ \\ $N, 6$ \\ $P(z), 9193$ \\ $P_{1}(z), 92$ \\ $P_{2}(z), 93$ \\ $P_{3}(z), 93,94$ \\ $P_{n}(z)$, 42 \\ $P_{n, 0}, 45$ \\ $R(z), 94$ \\ $S\left(\mu_{1}\right), 6$ \\ $S\left(\mu_{2}\right), 6$ \\ $S\left(\mu_{3}\right), 7$ \\ $S\left(\sigma_{2}-\mu_{2}\right), 6$ \\ $S(z), 70$ \\ $T(z), 65$ \\ $U(z), 53$ \\ $V$, 1 \\ $V_{1}, 5,1748$ \\ $V_{2}, 51$ \\ $V_{3}, 5,1748$ \\ $W, 1$ \\ $X(z), 45$
}

$Y(z), 11$

$\Gamma_{j}, 41$

$\Sigma_{A}, 90$

$\Sigma_{R}, 94$

$\Sigma_{S}, 70$

$\Sigma_{T}$, 65

$\Sigma_{j}, 76$

$\Theta(z), 16$

$\alpha, 1$

$\alpha_{k}$,31

$\ell_{1}, 53$

$\gamma_{2}, 61$

$\gamma_{3}, 61$

$\infty_{1}$, 79

$\infty_{2}, 79$

$\lambda_{j}, 38$

$\mathcal{R}, 34,76$

$\mathcal{R}_{j}, 34$

$\mathcal{S}$, [15]

$\mathcal{S}_{j}, 15$

$\mu_{j}$, 6

$\omega, 17,44$

$\omega_{P}, 79$

$\phi, 76$

$\pi, 76$

$\psi_{2}, 50$

$\psi_{3}, 49$

$\sigma_{2}, 550$

$\tau$, ,

$\theta_{j}, 16$

$\widehat{\gamma}_{2}, 69$

$\xi$, 35

$\xi_{j}, 34$

$a_{j}$, 6

$b_{j}$, 6

$c_{2}, 6$

$c_{3}, 7$

$f$, 92,93

$g_{j}$, 31

$p_{j, n}, 41$

$s_{j}$, 5] 15,16

$u_{j}, 82$

$v_{j}, 84$

$w_{j, n}, 11,41$ 
$x^{*}(\alpha), 5$

$y^{*}(\alpha), 6,15$

$\left(J_{M}\right)_{k}, 75$

$\left(J_{S}\right)_{1}, 71$

$\left(J_{S}\right)_{k}, 70$

$\left(J_{T}\right)_{k}, 66$

$\left(J_{U}\right)_{k}, 55,58$

$\left(J_{v}\right)_{k}, 84$

$J_{A}, 55$

$J_{M}, 75$

$J_{S}, 70$

$J_{T}, 66$

$J_{U}, 55,57$

$J_{v}, 84$

$J_{X}, 4950$

Airy function, 91

Airy kernel, 10

Airy RH problem, 90

asymptotics as $z \rightarrow \infty$

of $\lambda_{j}(z), 40$

of $\theta_{j}(z), 17$

of $\xi_{j}(z), 37$

of $F_{j}(z), 38$

of $g_{j}(z), 38$

of $P_{n}(z), 43,45$

of $s_{j}(z), 17$

of $U(z), 53$

of $u_{j}(z), 84$

of $v_{j}(z), 85$

of $X(z), 46$

balayage, 21

biorthogonal ensemble, 3

biorthogonal polynomials, 2 11

birth of a cut, 12

$B$-periods, 81

branch points, 89

canonical homology basis, 77

case I, $8,68,72,76$

case II, 8, 68, 72, 76, 77

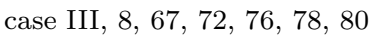

case IV, $8,12,72,76$

case V, 8, 72, 76, 79

Christoffel-Darboux formula, 11

conformal map $f$, 92,93

contours, 94

correlation kernel, 2,96

coupling constant, 1

cycles, 76, 77

de la Vallée Poussin theorem, 22

Deift-Zhou steepest descent, 3

density

$$
\begin{aligned}
& \text { of } \mu_{1}, 6 \\
& \text { of } \mu_{3}, 7 \\
& \text { of } \mu_{j}, 37 \\
& \text { of } \sigma_{2}-\mu_{2}, 6
\end{aligned}
$$

determinantal point process, 2 discrete orthogonal polynomials, 4 divisor, 86

energy functional, 4,27

equilibrium conditions

for $\nu_{1}, 25$

for $\nu_{2}, 26$

for $\nu_{3}, 23$

external field, 45, 17, 48,51.

Eynard-Mehta formula, 2

genus, 34,76

$g$-functions, 31

global parametrix, $12 \quad 75$

Hastings-McLeod solution, 12

Hermite functions, 12

inequalities for $\lambda_{j}, 40$

integrable kernel, 11

interlacing zeros, 2

intervals $\left[a_{j}, b_{j}\right], 6$

invariance of domain, 81

involution $\phi, 76$

iterated balayage, 26

jump properties

of $\lambda_{j}, 39$

of $g_{j}, 33$

of $P_{n}, 42$

of $X, 46$

of $g_{j}, 32$

of $u_{j}, 82$

of $v_{j}, 84$

lens, $61,63,68$

limiting mean eigenvalue distribution, 10

local parametrix, 89,91

local scaling limits, 10

logarithmic potential theory, 21

logarithmic energy, 3

logarithmic potential, 6

logarithmic potential theory, 3

marginal densities, 2

$M$-curve, 76

measures, 5, 6, 49, 50]

meromorphic differential, 79

meromorphic function, 35

minimizer, 6

model RH problem, 75

multi-critical behavior, 13

multiple orthogonal polynomial ensemble, 3

multiple orthogonal polynomials, 11

mutual logarithmic energy, 3

Nikishin system, 4

non-intersecting Brownian motions, 13

non-special divisor, 76 
one matrix model, 12

Painlevé $\mathrm{I}_{2}$ equation, 12

Painlevé II equation, 12

Painlevé II transition, 12

Pearcey integral, 41

Pearcey ODE, 41

Pearcey transition, 13

phase diagram, 8

phase diagram, 12

phase transitions, 8,12

point at infinity, 79

principle of domination, 21

projection $\pi, 76$

quartic equation, 36

quartic potential, 1

random matrices with external source, 13 recurrence relations, 8

regular $(V, W, \tau), 9$

regular cases, 7

residue conditions, 79

Riemann surface, 15, 34, 76

Riemann-Hilbert problem

for multiple orthogonal polynomials, 11

Riemann-Hilbert problem

for $M, 75$

for $P, 90$

for $R, 94$

for $S, 70$

for $T, 65$

for $U, 58$

for $X, 48$

for $Y$, 11

for Airy, 90

for biorthogonal polynomials, 3

Riemann-Roch theorem, 86

saddle point analysis, 43

saddle point equation, 5,15

sheets of the Riemann surface, 15, 34

sine kernel, 10

singular cases, $7 \longdiv { 1 2 }$

singular endpoint, 8

singular exterior point, 9

singular interior point, 8

singular supports, 812

spectral curve, 36

support, 6

of $\mu_{1}, 6$

of $\mu_{2}, 6$

of $\mu_{3}, 7$

of $\sigma_{2}-\mu_{2}, 6$

transformed functions, 2

two matrix model, 1

type II multiple orthogonality, 11

upper constraint, 4 variational conditions, 7

vector equilibrium problem, 3

weight functions, 11,41 


\section{Editorial Information}

To be published in the Memoirs, a paper must be correct, new, nontrivial, and significant. Further, it must be well written and of interest to a substantial number of mathematicians. Piecemeal results, such as an inconclusive step toward an unproved major theorem or a minor variation on a known result, are in general not acceptable for publication.

Papers appearing in Memoirs are generally at least 80 and not more than 200 published pages in length. Papers less than 80 or more than 200 published pages require the approval of the Managing Editor of the Transactions/Memoirs Editorial Board. Published pages are the same size as those generated in the style files provided for $\mathcal{A}_{\mathcal{M}} \mathcal{S}$-LATEX or $\mathcal{A} \mathcal{M} \mathcal{S}-\mathrm{T}_{\mathrm{E}} \mathrm{X}$.

Information on the backlog for this journal can be found on the AMS website starting from http://www.ams.org/memo.

A Consent to Publish and Copyright Agreement is required before a paper will be published in the Memoirs. After a paper is accepted for publication, the Providence office will send a Consent to Publish and Copyright Agreement to all authors of the paper. By submitting a paper to the Memoirs, authors certify that the results have not been submitted to nor are they under consideration for publication by another journal, conference proceedings, or similar publication.

\section{Information for Authors}

Memoirs is an author-prepared publication. Once formatted for print and on-line publication, articles will be published as is with the addition of AMS-prepared frontmatter and backmatter. Articles are not copyedited; however, confirmation copy will be sent to the authors.

Initial submission. The AMS uses Centralized Manuscript Processing for initial submissions. Authors should submit a PDF file using the Initial Manuscript Submission form found at www. ams.org/submission/memo, or send one copy of the manuscript to the following address: Centralized Manuscript Processing, MEMOIRS OF THE AMS, 201 Charles Street, Providence, RI 02904-2294 USA. If a paper copy is being forwarded to the AMS, indicate that it is for Memoirs and include the name of the corresponding author, contact information such as email address or mailing address, and the name of an appropriate Editor to review the paper (see the list of Editors below).

The paper must contain a descriptive title and an abstract that summarizes the article in language suitable for workers in the general field (algebra, analysis, etc.). The descriptive title should be short, but informative; useless or vague phrases such as "some remarks about" or "concerning" should be avoided. The abstract should be at least one complete sentence, and at most 300 words. Included with the footnotes to the paper should be the 2010 Mathematics Subject Classification representing the primary and secondary subjects of the article. The classifications are accessible from ww. ams.org/msc/. The Mathematics Subject Classification footnote may be followed by a list of key words and phrases describing the subject matter of the article and taken from it. Journal abbreviations used in bibliographies are listed in the latest Mathematical Reviews annual index. The series abbreviations are also accessible from www.ams.org/msnhtml/serials.pdf. To help in preparing and verifying references, the AMS offers MR Lookup, a Reference Tool for Linking, at www.ams.org/mrlookup/.

Electronically prepared manuscripts. The AMS encourages electronically prepared manuscripts, with a strong preference for $\mathcal{A} \mathcal{M} \mathcal{S}-\mathrm{L}_{\mathrm{A}} \mathrm{T} \mathrm{X}$. To this end, the Society has prepared $\mathcal{A} \mathcal{M S}$-LATEX author packages for each AMS publication. Author packages include instructions for preparing electronic manuscripts, samples, and a style file that generates the particular design specifications of that publication series. Though $\mathcal{A M S}_{\mathcal{M}}$ - $\mathrm{L}_{\mathrm{A}} \mathrm{T} \mathrm{X}$ is the highly preferred format of $\mathrm{T}_{\mathrm{E}} \mathrm{X}$, author packages are also available in $\mathcal{A} \mathcal{M} \mathcal{S}-\mathrm{T}_{\mathrm{E}} \mathrm{X}$.

Authors may retrieve an author package for Memoirs of the AMS from www.ams.org/ journals/memo/memoauthorpac.html or via FTP to ftp.ams.org (login as anonymous, enter your complete email address as password, and type cd pub/author-info). The 
AMS Author Handbook and the Instruction Manual are available in PDF format from the author package link. The author package can also be obtained free of charge by sending email to tech-support@ams . org or from the Publication Division, American Mathematical Society, 201 Charles St., Providence, RI 02904-2294, USA. When requesting an author package, please specify $\mathcal{A}_{\mathcal{M}} \mathcal{S}$ - $\mathrm{LT}_{\mathrm{E}} \mathrm{X}$ or $\mathcal{A}_{\mathcal{M S}} \mathrm{T}_{\mathrm{E}} \mathrm{X}$ and the publication in which your paper will appear. Please be sure to include your complete mailing address.

After acceptance. The source files for the final version of the electronic manuscript should be sent to the Providence office immediately after the paper has been accepted for publication. The author should also submit a PDF of the final version of the paper to the editor, who will forward a copy to the Providence office.

Accepted electronically prepared files can be submitted via the web at www.ams.org/ submit-book-journal/, sent via FTP, or sent on CD to the Electronic Prepress Department, American Mathematical Society, 201 Charles Street, Providence, RI 02904-2294 USA. TEX source files and graphic files can be transferred over the Internet by FTP to the Internet node ftp.ams.org (130.44.1.100). When sending a manuscript electronically via $C D$, please be sure to include a message indicating that the paper is for the Memoirs.

Electronic graphics. Comprehensive instructions on preparing graphics are available at www.ams.org/authors/journals.html. A few of the major requirements are given here.

Submit files for graphics as EPS (Encapsulated PostScript) files. This includes graphics originated via a graphics application as well as scanned photographs or other computergenerated images. If this is not possible, TIFF files are acceptable as long as they can be opened in Adobe Photoshop or Illustrator.

Authors using graphics packages for the creation of electronic art should also avoid the use of any lines thinner than 0.5 points in width. Many graphics packages allow the user to specify a "hairline" for a very thin line. Hairlines often look acceptable when proofed on a typical laser printer. However, when produced on a high-resolution laser imagesetter, hairlines become nearly invisible and will be lost entirely in the final printing process.

Screens should be set to values between $15 \%$ and $85 \%$. Screens which fall outside of this range are too light or too dark to print correctly. Variations of screens within a graphic should be no less than $10 \%$.

Inquiries. Any inquiries concerning a paper that has been accepted for publication should be sent to memo-query@ams . org or directly to the Electronic Prepress Department, American Mathematical Society, 201 Charles St., Providence, RI 02904-2294 USA. 


\section{Editors}

This journal is designed particularly for long research papers, normally at least 80 pages in length, and groups of cognate papers in pure and applied mathematics. Papers intended for publication in the Memoirs should be addressed to one of the following editors. The AMS uses Centralized Manuscript Processing for initial submissions to AMS journals. Authors should follow instructions listed on the Initial Submission page found at www.ams.org/memo/memosubmit.html.

Algebra, to ALEXANDER KLESHCHEV, Department of Mathematics, University of Oregon, Eugene, OR 97403-1222; e-mail: klesh@uoregon.edu

Algebraic geometry, to DAN ABRAMOVICH, Department of Mathematics, Brown University, Box 1917, Providence, RI 02912; e-mail: amsedit@math.brown.edu

Algebraic geometry and its applications, to MINA TEICHER, Emmy Noether Research Institute for Mathematics, Bar-Ilan University, Ramat-Gan 52900, Israel; e-mail: teicher@macs.biu.ac.il

Algebraic topology, to ALEJANDRO ADEM, Department of Mathematics, University of British Columbia, Room 121, 1984 Mathematics Road, Vancouver, British Columbia, Canada V6T 1Z2; e-mail: adem@math.ubc.ca

Automorphic forms, representation theory and combinatorics, to DAN BUMP, Department of Mathematics, Stanford University, Building 380, Sloan Hall, Stanford, California 94305; e-mail: bump@ math.stanford.edu

Combinatorics, to JOHN R. STEMBRIDGE, Department of Mathematics, University of Michigan, Ann Arbor, Michigan 48109-1109; e-mail: JRS@umich.edu

Commutative and homological algebra, to LUCHEZAR L. AVRAMOV, Department of Mathematics, University of Nebraska, Lincoln, NE 68588-0130; e-mail: avramov@math.unl.edu

Complex analysis and harmonic analysis, to MALABIKA PRAMANIK, Department of Mathematics, 1984 Mathematics Road, University of British Columbia, Vancouver, BC, Canada V6T 1Z2; e-mail: malabika@math.ubc.ca

Differential geometry and global analysis, to CHRIS WOODWARD, Department of Mathematics, Rutgers University, 110 Frelinghuysen Road, Piscataway, NJ 08854; e-mail: ctw@math.rutgers.edu

Dynamical systems and ergodic theory and complex analysis, to YUNPING JIANG, Department of Mathematics, CUNY Queens College and Graduate Center, 65-30 Kissena Blvd., Flushing, NY 11367; e-mail: Yunping.Jiang@qc.cuny.edu

Functional analysis and operator algebras, to NATHANIEL BROWN, Department of Mathematics, 320 McAllister Building, Penn State University, University Park, PA 16802; e-mail: nbrown@ math.psu.edu

Geometric analysis, to WILLIAM P. MINICOZZI II, Department of Mathematics, Johns Hopkins University, 3400 N. Charles St., Baltimore, MD 21218; e-mail: trans@math.jhu.edu

Geometric topology, to MARK FEIGHN, Math Department, Rutgers University, Newark, NJ 07102; e-mail: feighn@andromeda.rutgers.edu

Harmonic analysis, representation theory, and Lie theory, to E. P. VAN DEN BAN, Department of Mathematics, Utrecht University, P.O. Box 80 010, 3508 TA Utrecht, The Netherlands; e-mail: E.P.vandenBan@uu.nl

Logic, to ANTONIO MONTALBAN, Department of Mathematics, University of Chicago, 5734 S. University Avenue, Chicago, Illinois 60637; e-mail: antonio@math.uchicago.edu

Number theory, to SHANKAR SEN, Department of Mathematics, 505 Malott Hall, Cornell University, Ithaca, NY 14853; e-mail: ss70@cornell.edu

Partial differential equations, to GUSTAVO PONCE, Department of Mathematics, South Hall, Room 6607, University of California, Santa Barbara, CA 93106; e-mail: ponce@math.ucsb.edu

Partial differential equations and dynamical systems, to PETER POLACIK, School of Mathematics, University of Minnesota, Minneapolis, MN 55455; e-mail: polacik@math.umn.edu

Probability and statistics, to PATRICK FITZSIMMONS, Department of Mathematics, University of California, San Diego, 9500 Gilman Drive, La Jolla, CA 92093-0112; e-mail: pfitzsim@math.ucsd.edu

Real analysis and partial differential equations, to WILHELM SCHLAG, Department of Mathematics, The University of Chicago, 5734 South University Avenue, Chicago, IL 60615; e-mail: schlag@ math.uchicago.edu

All other communications to the editors, should be addressed to the Managing Editor, ROBERT GURALNICK, Department of Mathematics, University of Southern California, Los Angeles, CA 900891113; e-mail: guralnic@math.usc.edu. 


\section{Selected Titles in This Series}

1022 Maurice Duits, Arno B. J. Kuijlaars, and Man Yue Mo, The Hermitian two matrix model with an even quartic potential, 2012

1021 Arnaud Deruelle, Katura Miyazaki, and Kimihiko Motegi, Networking Seifert surgeries on knots, 2012

1020 Dominic Joyce and Yinan Song, A theory of generalized Donaldson-Thomas invariants, 2012

1019 Abdelhamid Meziani, On first and second order planar elliptic equations with degeneracies, 2012

1018 Nicola Gigli, Second order analysis on $\left(\mathscr{P}_{2}(\mathrm{M}), \mathrm{W}_{2}\right), 2012$

1017 Zenon Jan Jabłoński, Il Bong Jung, and Jan Stochel, Weighted shifts on directed trees, 2012

1016 Christophe Breuil and Vytautas Paškūnas, Towards a modulo p Langlands correspondence for $\mathrm{GL}_{2}, 2012$

1015 Jun Kigami, Resistance forms, quasisymmetric maps and heat kernel estimates, 2012

1014 R. Fioresi and F. Gavarini, Chevalley supergroups, 2012

1013 Kaoru Hiraga and Hiroshi Saito, On L-packets for inner forms of SL $n, 2012$

1012 Guy David and Tatiana Toro, Reifenberg parameterizations for sets with holes, 2012

1011 Nathan Broomhead, Dimer models and Calabi-Yau algebras, 2012

1010 Greg Kuperberg and Nik Weaver, A von Neumann algebra approach to quantum metrics/Quantum relations, 2012

1009 Tarmo Järvilehto, Jumping numbers of a simple complete ideal in a two-dimensional regular local ring, 2011

1008 Lee Mosher, Michah Sagee, and Kevin Whyte, Quasi-actions on trees II: Finite depth Bass-Serre trees, 2011

1007 Steve Hofmann, Guozhen Lu, Dorina Mitrea, Marius Mitrea, and Lixin Yan, Hardy spaces associated to non-negative self-adjoint operators satisfying Davies-Gaffney estimates, 2011

1006 Theo Bühler, On the algebraic foundations of bounded cohomology, 2011

1005 Frank Duzaar, Giuseppe Mingione, and Klaus Steffen, Parabolic systems with polynomial growth and regularity, 2011

1004 Michael Handel and Lee Mosher, Axes in outer space, 2011

1003 Palle E. T. Jorgensen, Keri A. Kornelson, and Karen L. Shuman, Iterated function systems, moments, and transformations of infinite matrices, 2011

1002 Man Chun Leung, Supported blow-up and prescribed scalar curvature on $S^{n}, 2011$

1001 N. P. Strickland, Multicurves and equivariant cohomology, 2011

1000 Toshiyuki Kobayashi and Gen Mano, The Schrödinger model for the minimal representation of the indefinite orthogonal group $\mathrm{O}(\mathrm{p}, \mathrm{q}), 2011$

999 Montserrat Casals-Ruiz and Ilya Kazachkov, On systems of equations over free partially commutative groups, 2011

998 Guillaume Duval, Valuations and differential Galois groups, 2011

997 Hideki Kosaki, Positive definiteness of functions with applications to operator norm inequalities, 2011

996 Leonid Positselski, Two kinds of derived categories, Koszul duality, and comodule-contramodule correspondence, 2011

995 Karen Yeats, Rearranging Dyson-Schwinger equations, 2011

994 David Bourqui, Fonction zêta des hauteurs des variétés toriques non déployées, 2011

993 Wilfrid Gangbo, Hwa Kil Kim, and Tommaso Pacini, Differential forms on Wasserstein space and infinite-dimensional Hamiltonian systems, 2011

992 Ralph Greenberg, Iwasawa theory, projective modules, and modular representations, 2011

991 Camillo De Lellis and Emanuele Nunzio Spadaro, Q-valued functions revisited, 2011 
990 Martin C. Olsson, Towards non-abelian p-adic Hodge theory in the good reduction case, 2011

989 Simon N. Chandler-Wilde and Marko Lindner, Limit operators, collective compactness, and the spectral theory of infinite matrices, 2011

988 R. Lawther and D. M. Testerman, Centres of centralizers of unipotent elements in simple algebraic groups, 2011

987 Mike Prest, Definable additive categories: Purity and model theory, 2011

986 Michael Aschbacher, The generalized fitting subsystem of a fusion system, 2011

985 Daniel Allcock, James A. Carlson, and Domingo Toledo, The moduli space of cubic threefolds as a ball quotient, 2011

984 Kang-Tae Kim, Norman Levenberg, and Hiroshi Yamaguchi, Robin functions for complex manifolds and applications, 2011

983 Mark Walsh, Metrics of positive scalar curvature and generalised Morse functions, part I, 2011

982 Kenneth R. Davidson and Elias G. Katsoulis, Operator algebras for multivariable dynamics, 2011

981 Dillon Mayhew, Gordon Royle, and Geoff Whittle, The internally 4-connected binary matroids with no $\mathrm{M}\left(\mathrm{K}_{3,3}\right)$-Minor, 2010

980 Liviu I. Nicolaescu, Tame flows, 2010

979 Jan J. Dijkstra and Jan van Mill, Erdős space and homeomorphism groups of manifolds, 2010

978 Gilles Pisier, Complex interpolation between Hilbert, Banach and operator spaces, 2010

977 Thomas Lam, Luc Lapointe, Jennifer Morse, and Mark Shimozono, Affine insertion and Pieri rules for the affine Grassmannian, 2010

976 Alfonso Castro and Víctor Padrón, Classification of radial solutions arising in the study of thermal structures with thermal equilibrium or no flux at the boundary, 2010

975 Javier Ribón, Topological classification of families of diffeomorphisms without small divisors, 2010

974 Pascal Lefèvre, Daniel Li, Hervé Queffélec, and Luis Rodríguez-Piazza, Composition operators on Hardy-Orlicz space, 2010

973 Peter O'Sullivan, The generalised Jacobson-Morosov theorem, 2010

972 Patrick Iglesias-Zemmour, The moment maps in diffeology, 2010

971 Mark D. Hamilton, Locally toric manifolds and singular Bohr-Sommerfeld leaves, 2010

970 Klaus Thomsen, $C^{*}$-algebras of homoclinic and heteroclinic structure in expansive dynamics, 2010

969 Makoto Sakai, Small modifications of quadrature domains, 2010

968 L. Nguyen Van Thé, Structural Ramsey theory of metric spaces and topological dynamics of isometry groups, 2010

967 Zeng Lian and Kening Lu, Lyapunov exponents and invariant manifolds for random dynamical systems in a Banach space, 2010

966 H. G. Dales, A. T.-M. Lau, and D. Strauss, Banach algebras on semigroups and on their compactifications, 2010

965 Michael Lacey and Xiaochun Li, On a conjecture of E. M. Stein on the Hilbert transform on vector fields, 2010

964 Gelu Popescu, Operator theory on noncommutative domains, 2010

963 Huaxin Lin, Approximate homotopy of homomorphisms from $\mathrm{C}(\mathrm{X})$ into a simple $C^{*}$-algebra, 2010 
I SBN 978-0-8218-6928-4 